\title{
AMMI Canada-CACMID 2008 Annual Conference February 27 - March 2, 2008 Sheraton Vancouver Wall Centre Hotel, Vancouver, British Columbia
}

\begin{tabular}{cc}
\hline AUTHOR LEGEND \\
\hline Asterisk $(*)$ & Presenting author \\
\hline $\begin{array}{c}\text { Oral Presentations } \\
\text { Thursday, February 28 }\end{array}$
\end{tabular}

Session A

A1 DO BUGS NEED DRUGS? - FOCUS ON PHYSICIANS -
THREE EVIDENCE-BASED STRATEGIES TO LIMIT YOUR
"RESISTANCE FOOTPRINT" D Patrick ${ }^{1,2 *}$, E Blondel-Hill', F Marra ${ }^{1,2}$, D Purych ${ }^{3}$, S Dobson'1, W Bowie ${ }^{1}$, I Vrbova ${ }^{1}$, A Chau ${ }^{1}$, B Henry ${ }^{1,2}$, P Kendall ${ }^{1}$, M Chong ${ }^{1,2}$, K Dreher ${ }^{1,2}$, M Carson ${ }^{4}$, D Low ${ }^{5}$

${ }^{1}$ University of British Columbia; ${ }^{2}$ British Columbia Centre for Disease Control, Vancouver; ${ }^{3}$ Fraser Health Authority, Surrey, BC; ${ }^{4}$ Captial Health Authority, Edmonton, AB; ${ }^{5}$ University of Toronto, Toronto, ON

OBJECTIVES: Antibiotic resistance is driven by natural selection associated with antibiotic utilization and clonal spread. Since 2005, the government of BC has funded the Do Bugs Need Drugs? Program to address overuse of antibiotics in the community. The program is currently focusing on assisting physicians to reduce their "resistance footprint" by crafting clear messages from current evidence.

METHODS: Trends in antimicrobial utilization were derived from IMS Health Canada and BC PharmaNet data and expressed in defined daily doses (DDD). Conditions associated with prescription were obtained through BC Medical Services Plan. Trends in resistance were tracked through the Canadian Bacterial Surveillance Network and BC Biomedical Laboratories. The literature was reviewed for ecological, cohort and prospective studies of factors associated with antibiotic resistant infections. RESULTS: While utilization of antimicrobials, including newer macrolides and fluoroquinolones has levelled off in BC since 2005, no overall decline in utilization is observed. Pneumococcal macrolide nonsusceptibility from BC sources ranged from 14-23\% during 2006-2007. $>50 \%$ of macrolide use was linked to treatment of upper respiratory infections. Published ecological and cohort studies strongly support the observation that azithromycin is a more potent selector for resistance than shorter half life macrolides (relative risk or odds ratios of 3.31-9.93). Most guidelines now urge watch and wait for otitis media and no treatment for acute bronchitis. Fluoroquinolone non-susceptibility in E. coli and other uropathogens continues to climb rapidly (22\% in E. coli in 2007 in BC) and is strongly associated with increased use of fluoroquinolones $(p<0.01)$ Resistance to nitrofurantoin remains very low at $3 \%$ among $E$. coli isolates. CONCLUSIONS: As part of its educational initiatives for physicians in 2007/8, the Do Bugs Need Drugs? Program will target three key evidence based strategies to address antimicrobial resistance 1) Avoid macrolides, especially azithromycin, for respiratory tract infections-consider tetracyclines instead for lower respiratory tract infections, 2) Avoid antibiotic therapy for acute bronchitis and early otitis media; 3) Avoid fluoroquinolones as first line therapy for uncomplicated cystitis - consider nitrofurantoin instead.

\section{A2 \\ EXTENDED-SPECTRUM $\beta$-LACTAMASE-PRODUCING ESCHERICHIA COLI FROM CANADIAN ICUS ARE SUSCEPTIBLE TO MECILLINAM}

P Lagacé-Wiens $^{1 *}$, P Baudry ${ }^{1}$, M Decorby ${ }^{1}, \mathrm{~K} \mathrm{Nichol}^{2}$, D Hoban ${ }^{1,2}$, G Zhanel ${ }^{1,2}$

${ }^{1}$ University of Manitoba, Department of Medical Microbiology;

${ }^{2}$ Clinical Microbiology, Health Sciences Centre, Winnipeg, MB

OBJECTIVE: Mecillinam is a $\beta$-lactam antibiotic with high affinity to the penicillin binding protein PBP- 2 of gram-negative organisms and relative stability to hydrolysis by the $\beta$-lactamases of gram-negative bacteria including some Extended-Spectrum $\beta$-Lactamases (ESBLs). The antibiotic is also available in a formulation that is orally bioavailable (pivmecillinam) and thus may be an option for the outpatient treatment of infections caused by ESBL-producing organisms. The purpose of this study was to determine the mecillinam susceptibility of ESBL-producing organisms isolated from Canadian intensive care units.

METHODS: The antimicrobial susceptibility of $536 \mathrm{E}$. coli isolates from

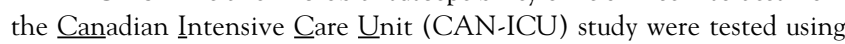
broth microdilution. Isolates with reduced susceptibility to ceftriaxone $(\mathrm{MIC} \geq 1 \mathrm{~g} / \mu \mathrm{l})$ were tested for ESBL production using the CLSI disk diffusion method. Isolates with confirmed ESBL phenotypes were subject to PCR amplification and sequencing of bla $a_{\text {CTX-M }}$, bla $a_{\text {SHV }}$ and bla $a_{\text {TEM }}$ genes to determine the ESBL gene responsible for resistance. ESBL producing isolates were tested for susceptibility to mecillinam using 10 and $25 \mu \mathrm{g}$ mecillinam disks and results were interpreted according to CLSI guidelines.

RESULTS: Of 536 E. coli isolates, 19 (3.5\%) were ESBL-producing isolates. There were 14 (73.7\%) CTX-M-15, 2 (10.5\%) CTX-M-2 and 1 (5.3\%) each of CTX-M-9, CTX-M-14 and CTX-M-1 producing isolates. No SHV or TEM genes whose products were known to be ESBLs were identified. All isolates were susceptible to mecillinam using CLSI disk diameter breakpoints. Mean and median disk diameters for the $10 \mu \mathrm{g}$ disk were $24.1 \mathrm{~mm}$ and $25 \mathrm{~mm}$, and $27 \mathrm{~mm}$ and $27 \mathrm{~mm}$ for the $25 \mu \mathrm{g}$ disks, respectively. Maximum disk diameters were 30 and $31 \mathrm{~mm}$ and minimum diameters were $19 \mathrm{~mm}$ and $23 \mathrm{~mm}$ for the $10 \mu \mathrm{g}$ and $25 \mu \mathrm{g}$ disk, respectively. The CTX-M gene was not associated with the disk diameter.

CONCLUSIONS: All ESBL producing E. coli isolates from the CANICU study were susceptible to mecillinam using CLSI breakpoints. Mecillinam and its oral formulation pivmecillinam may be a therapeutic option for infections caused by ESBL-producing E. coli.

\section{A3 DETERMINANTS OF REGIONAL VARIATION IN OUTPATIENT ANTIBIOTIC CONSUMPTION IN BRITISH COLUMBIA}

F Marra $^{1,2}$, S Mak ${ }^{1 *}$, M Chong1, D Patrick ${ }^{1,3}$

${ }^{1}$ British Columbia Centre for Disease Control; ${ }^{2}$ University of British Columbia, Faculty of Pharmaceutical Sciences; ${ }^{3}$ University of British Columbia, Department of Health Care and Epidemiology, Vancouver, BC

OBJECTIVES: Antibiotic consumption in human populations is a factor in the emergence of resistant organisms. As such, we tracked populationbased consumption data on an ongoing basis to explore the determinants for regional variations in antibiotic consumption for the province of British Columbia (BC).

METHODS: We obtained data from the BC PharmaNet database on all 
outpatient oral antimicrobial prescriptions for 1996-2005. Prescriptions were expressed as their defined daily dose (DDD) per 1,000 inhabitants according to the 2006 World Health Organization Anatomical Therapeutic Chemical system. We explored the relationships between antimicrobial consumption and socioeconomic and climatic factors using Pearson's correlation. Geographic Information Systems mapping was used to display the spatial variations of antibiotic consumption in BC. Overall and class-specific rates of consumption were described by the Health Service Delivery Area geography.

RESULTS: Overall antibiotic consumption was highest in the northern health regions and lowest in the interior health regions. Correlations were found between antibiotic consumption and socioeconomic and climatic factors. Higher rates of antibiotic consumption were associated with the aboriginal population, higher family income, and July total precipitation. An inverse relationship was found between consumption and July average temperature. Further analysis of class-specific antibiotic consumption identified different geographic patterns of consumption and socioeconomic associations. Higher rates of penicillin, beta-lactam, and macrolide consumption were seen with the aboriginal population, younger population (age $<15$ years), higher ratio of physicians to population, higher family income, and greater July total precipitation. An inverse correlation was found between antibiotic consumption and older age (age $>65$ years), mortality rate, and warmer July temperatures.

CONCLUSIONS: Different rates of antibiotic consumption exist within the province. Appropriate policies affecting antibiotic consumption in the community can be designed by looking at the relationships between antibiotic consumption and socioeconomic determinants, and their related impact.

\section{A4}

A REAL-TIME PCR ASSAY FOR DETECTION OF VANCOMYCIN RESISTANCE GENES IN ENTEROCOCCI USING BHQPLUS ${ }^{\text {TM }}$ TAQMAN PROBES AND THE SPARTAN DX $^{\text {TM }}$ INSTRUMENT

N Arbour $^{1 *}$, C Harder ${ }^{1}$, B Toye ${ }^{1,2}$, K Ramotar $^{1,2}$

${ }^{1}$ The Ottawa Hospital Research Institute; ${ }^{2}$ The Ottawa Hospital, Ottawa, ON

OBJECTIVES: Identification of vancomycin-resistant enterococci (VRE) is important to limit nosocomial spread and has traditionally relied on phenotypic methods. A rapid real-time PCR assay detecting vanA, van $B$ or vanC resistance genes was developed for use on a new instrument using a new probe technology. The Spartan DX ${ }^{\mathrm{TM}}$ is a singleplex real-time PCR instrument, with four wells, designed for on-demand, non-batched applications where speed and convenience are important. BHQPlus ${ }^{\mathrm{TM}}$ is a probe chemistry that increases probe melting temperature and enables more sensitive fluorescence detection. The purpose of this study was to evaluate the Spartan DX and BHQPlus probes to detect and genotype VRE isolates.

METHODS: Three sets of primers with corresponding BHQPlus TaqMan ${ }^{\circledR}$ probes or regular TaqMan probes were designed against the same conserved regions of the $\operatorname{van} \mathrm{A}$, $\operatorname{van} B, \operatorname{van} \mathrm{B} 2 / 3$, vanC1 and $\operatorname{van} \mathrm{C} 2 / \mathrm{C} 3$ genes. To date, the isolates tested include 26 enterococci with either vanA or vanB, 7 with vanC and 10 non-enterococci (including S. aureus, E. coli, coag-negative Staphylococcus, group C and G Streptococcus). Amplification was performed from crude DNA lysates. Real-time PCR was performed using both regular TaqMan probes and BHQPlus TaqMan probes to vanA, van $B$ and vanC, to genotype the isolates. The specificity and efficiency of the assay was validated by performing serial dilutions and examining crossreactivity by PCR and gel electrophoresis.

RESULTS: Our preliminary results indicate that BHQPlus probes specifically identified $v a n A, v a n B$, vanB2/B3, vanC1 and vanC2/C3 genes from all tested enterococcus isolates without cross-reactivity between the van genes. The regular Taqman probes only recognized the $\operatorname{van} A$, van $B$ and vanB2/B3 genes. Efficient real-time amplification was detected with serially diluted samples down to the 1-10 copy range. In addition, none of the VRE BHQPlus primer sets cross-reacted with non-enterococcus lysates tested.
CONCLUSION: These preliminary results demonstrate specific and rapid identification of $\operatorname{van} A$, $\operatorname{van} B$ or $\operatorname{van} C$ genes in enterococci from crude lysates. Use of BHQPlus probes results in increased reaction sensitivity, and allowed for the creation of a more effective and efficient real-time PCR assay.

\section{Session B}

B1

\section{COMPARISON OF TWO NEW DFA ASSAYS TO RT-PCR FOR THE DETECTION OF HUMAN METAPNEUMOVIRUS IN} RESPIRATORY SPECIMENS

A Petrich ${ }^{1 *}$, M Booth ${ }^{1}, \mathrm{~K}$ Luinstra ${ }^{1}, \mathrm{M} \mathrm{Ackerman}^{1}{ }^{1}, \mathrm{~S}_{\text {Castriciano }}{ }^{2}$, M Smieja ${ }^{1}$, J Mahony ${ }^{1}$

${ }^{1}$ St. Joseph's Healthcare, Hamilton, ON; ${ }^{2}$ Copan Italia, Brescia, Italy

OBJECTIVE: Human Metapneumovirus (hMPV) was first identified in 2001 and is now recognized as a significant cause of upper and lower respiratory tract infections, particularly in young children. The gold standard for detection of the virus has been RT-PCR, although this is not routinely available in most virology laboratories. Two new commercially available direct DFA assays have been developed. In this study, we compare the performance of these assays to RT-PCR for the detection of hMPV in respiratory specimens.

METHODS: 204 nasopharyngeal swab (NPS) specimens were collected from May 7 to June 3, 2007 from patients ranging in age from 8 days to 95 years old. All specimens were collected in Copan UTM-RT with a flocked swab. A $200-500 \mathrm{uL}$ aliquot of vortexed specimen was stored at $-70^{\circ} \mathrm{C}$ for nucleic acid extraction and RT-PCR testing. The remainder of the sample was centrifuged and two slides were spotted with the cell pellet. After fixing, the slides were stained with two direct hMPV immunofluorescent assays, one from Diagnostic Hybrids Inc. and one from Chemicon (now Millipore Corporation). RT- PCR was performed using an in-house assay targeting the nucleoprotein gene (Maertzdorf et al., 2004). The stored aliquot was thawed and extracted using EasyMag (bioMérieux). Amplification products were separated on a $2.0 \%(\mathrm{w} / \mathrm{v})$ agarose gel and visualized with ethidium bromide staining and UV-light detection.

RESULTS: Thirteen specimens were positive in all assays, with 18 samples positive in 2 or more assays. RT-PCR detected 17 of these positives plus 2 additional positives for a sensitivity and specificity of $94.4 \%$ and $98.9 \%$ respectively. The Chemicon DFA detected $18 / 18$ positives with no additional positives for a sensitivity and specificity of $100 \%$. The DHI DFA detected $15 / 18$ positives for a sensitivity of $83.3 \%$ and a specificity of $100 \%$.

CONCLUSION: The DFA assays had similar sensitivity to the RT-PCR for the detection of hMPV and would be a viable alternative to RT-PCR testing in clinical laboratories.

\section{B2}

OF GASTRO AND THE GOLD STANDARD: USE OF LATENT CLASS MODELLING TO ESTIMATE TEST PERFORMANCE FOR A NOVEL PCR AND EIA FOR NOROVIRUS G1 AND G2 D Fisman ${ }^{1,2 *}$, G Brouhanski ${ }^{1}$, T Mazzulli ${ }^{1,3}$, S Drews ${ }^{1,3}$

${ }^{1}$ Ontario Central Public Health Laboratory; ${ }^{2}$ Research Institute of the Hospital for Sick Children; ${ }^{3}$ Mt. Sinai Hospital, Toronto, ON

BACKGROUND: The norovirus group (NVG) of Caliciviruses are the etiological agents of most institutional outbreaks of gastroenteritis in North America and Europe. In the frail elderly NVG outbreaks are associated with significant morbidity and mortality. Identification of NVG is complicated by the non-culturable nature of this virus, and the absence of a diagnostic gold standard makes traditional evaluation of test characteristics problematic. Latent class regression models provide a means for estimating sensitivity and specificity when a gold standard is lacking.

METHODS: The Ontario Central Public Health Laboratory serves as the major diagnostic facility for identification of NVG outbreaks in Ontario hospitals, long-term care facilities, and schools. Parallel testing with both 
RT-PCR and electron microscopy (EM) for the identification of NV in clinical specimens from all institutional outbreaks of diarrhoea and vomiting has been performed since August 2006; additional EIA testing was performed on 188 specimens. Such parallel testing allowed creation of a latent class regression model, which used covariance-based estimation to assign true or false positive status to groupings of test results. Test characteristics were calculated relative to this latent classification.

RESULTS: 14 specimens were EM positive; all were positive by PCR, and 12/14 by EIA. Among EM negative specimens, 83/170 (49\%) were positive by either PCR or EIA, (74/83 (93\%) PCR-positive; 60/83 (72\%) EIApositive). Latent class modelling estimated sensitivities of PCR, EIA, and EM as $100 \%, 86 \%$, and $17 \%$ respectively; specificities were $84 \%, 92 \%$, and $100 \%$. A single appropriate specimen will identify a NVG outbreak by PCR, 7 specimens are needed for identification by EIA with $>95 \%$ certainty, while 16 specimens for identification by EM with $>95 \%$ certainty. False positive identification of NVG with a probability $>50 \%$ will occur with testing of $>4$ negative specimens by PCR or EIA.

CONCLUSIONS: Latent class methods support the characterization of EM as lacking sensitivity for NVG outbreaks. The high sensitivity of PCR permits identification of NVG with high sensitivity on limited numbers of clinical specimens. Testing large numbers of specimens with PCR or EIA will falsely identify NVG as the etiologic agent in non-NVG gastroenteritis outbreaks. For this reason, it is reasonable to limit the number of specimens tested per outbreak.

\section{B3}

DUAL $\boldsymbol{H}$ INFLUENZAE AND RESPIRATORY SYNCYTIAL VIRUS: IS DETECTION OF TWO ORGANISMS ASSOCIATED WITH INCREASED SEVERITY OF RESPIRATORY ILLNESS? L Sauve $^{1 *}$, M Al-Dabbagh ${ }^{1}$, E Thomas ${ }^{1}$, S Dobson ${ }^{1}$, J Brunstein ${ }^{1,2}$ ${ }^{1}$ University of British Columbia; ${ }^{2}$ Centre for Translational and Applied Genomics, Vancouver, BC

OBJECTIVE: The clinical significance of coinfections with multiple respiratory pathogens in pediatrics is not entirely known, although there is some data to suggest that those children may be more severely ill. The introduction of respiratory multiplex assays in diagnostic laboratories, will more frequently detect multiple agents than classical methods. We aimed in this study to determine if children with clinical bronchiolitis and two organisms detected (H Influenzae and Respiratory Syncytial Virus) by a multiplex assay are more severely ill than children with a single organism.

METHODS: A retrospective case control study compared children with only H. Influenzae or RSV, or both H Influenzae and RSV was done. In an evaluation of the Qiaplex Resplex I and II in the 2006 - 2007 respiratory virus season, 1742 clinical specimens were tested. A sample of positive specimens (9 H Influenzae alone, 5 RSV alone, and 18 H Influenzae plus RSV) was selected for chart review with a standardized form. Severity of illness was based on duration of hospitalization, intensive care admission, and need for assisted ventilation.

RESULTS: Thirty-two children were included, with $56 \%$ boys. $44 \%$ had one pathogen detected (either H. influenzae or RSV). The mean age was 17.5 months; excluding two outliers (ages 6 and 8 years) the mean age of the two groups was the same. Twelve children $(37 \%)$ had underlying conditions that put them at higher risk of severe bronchiolitis; those children were significantly less likely to be admitted to hospital. There were no significant differences between the groups dual vs single pathogens for admission to hospital (OR 1.95, 95\% CI 0.35 - 11). Even without the microbiological data available to the treating physician, none of the five children with RSV alone, and $48 \%$ of those with $H$ influenzae (alone or with RSV) did, however it was not significant.

CONCLUSIONS: Children with two pathogens found on molecular testing were not significantly different from those with one pathogen (and no differences were statistically significant). This result contrasts with earlier suggestions that children with $H$ Influenzae found on multiplex PCR specimens may be more severely ill. However, this result may be due to the small number of cases.

\section{B4}

RESULTS OF A WEST NILE VIRUS (WNV) RNA PROFICIENCY PANEL SENT TO CANADIAN LABORATORIES A Petrich ${ }^{1 *}$, K Luinstra ${ }^{1}$, A Dibernardo ${ }^{2}$, $\mathrm{R}$ Lindsay ${ }^{2}, \mathrm{M} \mathrm{Drebot}^{2}$ and National Molecular Microbiology Diagnostics Users Group (NMG) 'St Joseph's Healthcare, McMaster University, Hamilton, ON; ${ }^{2}$ National Microbiology Laboratory (NML), Public Health Agency of Canada (PHAC), Winnipeg, MB

BACKGROUND: Members of the NMG identified that external quality proficiency programs did not provide WNV RNA proficiency samples. The NMG worked with NML to provide panels for nucleic acid proficiency testing by Canadian laboratories.

OBJECTIVES: 1 . To describe the set up and testing of a WNV RNA proficiency panel. 2. To discuss the results for the WNV RNA proficiency panel sent out by the NMG to participating laboratories.

METHODS: The panel consisted of ten $1 \mathrm{~mL}$ serum samples. Serum was obtained from 5 "volunteers" and was pooled following centrifugation. Pooled serum was tested to ensure it was WNV negative prior to spiking. Heat-inactivated viral lysates (WNV NY99, dengue 2 virus, St. Louis Encephalitis virus [SLEV]) were obtained from the NML. WNV lysate was titrated to determine an approximate end-point by real time RT-PCR. Four WNV samples were generated for the panel $\left(2\right.$ at $10^{3}, 1$ at $10^{4}$, and 1 at $10^{5} \mathrm{pfu} / \mathrm{mL}$ ). Four samples had no virus added and there was 1 sample each with dengue 2 virus and SLEV (10 pfu/mL each) added for a total of 10 samples/panel. Samples were frozen at $-80^{\circ} \mathrm{C}$. Twelve laboratories received panels shipped on dry ice. Laboratories reported their results following testing of the panels and filled in a questionnaire to describe their testing methodology.

RESULTS: Five different extraction methods were used including manual and automated, and RNA and total nucleic acid methods. Two different commercial assays and 5 different in-house assays were used. Despite the different methods for extraction and amplification, all laboratories reported the correct samples as positive. All laboratories provided correct identification of WNV samples. No false positives were detected. Low level positives were weak and tested the systems.

CONCLUSIONS: The proficiency panel developed by the NMG and NML was an effective challenge of the testing methods used in Canadian laboratories for detection of WNV RNA in serum samples. Future panels will be developed for agents not currently covered by external proficiency panel programs.

\section{Session C}

\section{C1}

FUNGAL IDENTIFICATION BY SEQUENCING OF THE INTERNAL TRANSCRIBE SPACER REGIONS AND 5.8S rRNA SUBUNIT

A McNabb ${ }^{1 *}$, G Geddes ${ }^{1}$, S Mithani ${ }^{1}$, L Hoang ${ }^{1,2}$, D Eisler ${ }^{1}$, P Tang ${ }^{1,2}$ ${ }^{1}$ British Columbia Centre for Disease Control Laboratory Services; ${ }^{2}$ Dept of Pathology and Laboratory Medicine, University of British Columbia, Vancouver, BC

OBJECTIVE: To determine the practicality of using DNA sequencing of the Internal Transcribed Spacer Regions, ITS1 and ITS2, and the 5.8S rRNA subunit (ITS) for the identification of fungi by comparing classical identification $(\mathrm{CI})$ to sequence-based identification.

METHODS: 108 type, reference and clinical strains of yeast and fungi had their DNA extracted using a published modified Ultraclean Microbial DNA Isolation $\mathrm{kit}^{\mathrm{TM}}$ protocol. The region between the $18 \mathrm{~S}$ and $28 \mathrm{~S}$ rRNA subunits was amplified and sequenced using primers ITS1 and ITS4. Generated sequence was compared to the NCBI database. Identifications to genus or species level were based on consideration of the maximum bit score, percent coverage and E-Value of the BLASTn alignments.

RESULTS: The fungal extraction protocol had more PCR amplification failures from molds than yeasts with only $46 / 108$ isolates (42\%) yielding a PCR product and usable DNA sequence. Of the 46 isolates, ITS sequencing confirmed the $\mathrm{CI}$ in 40 isolates (87\%) either identifying the isolate as 
the same genus and species or as the species' telomorph. In 4 cases ITS sequencing was able to identify an isolate to a lower taxonomic level than CI methods. The 6 discrepant identifications included one isolate identified as C. stellatoidea by CI and C. albicans by ITS, one isolate as A. niger by $\mathrm{CI}$ and A. brasiliensis by ITS, one isolate as T. rubrum by CI and T. mentagrophytes by ITS, one isolated queried as Acremonium sp. by CI and Paecilomyces sp. by ITS and one isolate as T. mentagrophytes by CI and T. rubrum by ITS.

CONCLUSION: Our lysis method requires further optimization to improve the yield of high quality DNA for PCR and sequencing. We speculate that PCR failures are likely due to insufficient DNA extracted from the isolates or inhibitors present in the extract. For fungal species whose DNA could be amplified, ITS sequencing appears to be a rapid and reliable means of identifying an expanded spectrum of fungal species from clinical sources. However, for this method to be used with confidence, a comprehensive and curated database is required to be certain that the identification generated by ITS sequencing is accurate.

\section{C2}

CHARACTERIZATION OF TWO POLYKETIDE SYNTHASE GENES INVOLVED IN THE BIOSYNTHESIS OF PENICILLIC ACID, ASPERLACTONE AND ISOASPERLACTONE IN ASPERGILLUS WESTERDIJKIAE NRRL 3174

N Bacha, H Phong Dao, F Mathieu, T Liboz, A Lebrihi Institut National Polytechnique de Toulouse, Toulouse, France OBJECTIVE: Penicillic acid (PA), asperlactone (AL) and isoasperlactone (IAL) are pharmacologically important secondary metabolites; they belong to a structurally diverse group with a broad range of biological activities known as polyketides. Polyketide syntheses are responsible for the biosynthesis of these metabolites. Various polyketide Synthase genes have been identified in Aspergillus westerdijkiae NRRL 3174, but none of the genes responsible for the production of these toxic pharmacologically important metabolites have been characterized. This study is not only important for characterization of the target genes; it also can be used as a base for identifying PA, AL and IAL producers and non-producers of fungi through rapid PCR based technique.

METHODS: Modified double joint PCR technique was used for the construction of disruption vector, and modified protoplast based transformation technique was used for disruption of the target gene. The transformants were screened initially on Hygromycine containing rich media, then through PCR approach and finally through southern blot hybridization. The confirmed mutants were analyzed for morphological and metabolical deficiencies by comparing their HPLC profiles with the wild type. Through genetic complementation, role of each gene in the biosynthetic process was confirmed.

RESULTS: Two polyketide synthase genes AoMSAS (5.29 kb) and AoLC35-6 (1.9 kb) involved in the biosynthesis of PA, AL and IAL were cloned by using a "gene walking" strategy. The predicted amino acid sequence of AoMSAS shows about 50\% identity to 6-methylsalicylic acid synthases from A. terreus and P. patulum while AoLC35-6 shows about $60 \%$ identity to hybrid PKS/NRPS of A. niger, A. clavatus and Magnaporthe grisea. The expression of AoMSAS genes and AoLC35-6, and polyketide production were also investigated in A. westerdijkiae. The mutants of A. westerdijkiae in which the AoMSAS gene was disrupted could not produce AL and IAL, while no effect on the production of PA was observed. On the other hand mutants in which AoLC35-6 gene was disrupted lost the capability to produce PA, AL and IAL. However, no visible change in fungal growth, sporulation, or pigment production was observed. Complementary mutations of both PKS restored production of mixing metabolites and confirmed the role of the AoMSAS gene in the biosynthesis of AL and IAL and that of AoLC35-6 in the production of PA,AL and IAL

CONCLUSION: Two hypothesis can be concluded: 1 - All three metabolites are produced through the same biosynthetic pathway, in which AoLC35-6 gene results into the production of a common precursor for all the three metabolites while AoMSAS gene results into an intermediate which is not involved in PA biosynthesis. 2- PA and Asperlactone/
Isoasperlactone are produced through two different biosynthetic pathways in which AoLC35-6 results into a product responsible for the biosynthesis of all the three metabolites.

\section{C3}

\section{CASPOFUNGIN ETEST ENDPOINT FOR ASPERGILLUS SHOWS POOR ESSENTIAL AGREEMENT WITH THE REFERENCE MINIMUM EFFECTIVE CONCENTRATION (MEC)}

A Schofield, C Sand, L Breitkreutz, L Turnbull, R Rennie, J Fuller* Division of Medical Microbiology, University of Alberta Hospital, Edmonton, AB

OBJECTIVE: The minimum effective concentration (MEC) is the currently accepted broth microdilution (BMD) endpoint for measuring echinocandin activity against filamentous fungi. However, this method requires microscopic examination of fungal growth, which prevents many laboratories from adopting this methodology. We evaluated the caspofungin Etest as an alternative to the BMD for MEC determination in Aspergillus.

METHODS: BMD was performed according to the Clinical Laboratories Standards Institute (CLSI) document M38-A using 0.016 to $32 \mathrm{mg} / \mathrm{L}$ caspofungin. MEC was determined microscopically for the first 30 specimens, correlated to the macroscopic appearance of each well, and then inferred from macroscopic appearance for remaining isolates. Etest was performed using a modification of the CLSI M44-A disk diffusion method on Mueller-Hinton agar supplemented with glucose $(2 \%)$ and methylene blue $(0.5 \mathrm{mg} / \mathrm{L})$. Caspofungin Etest endpoints were measured at the intersection between Aspergillus growth and the Etest strip. All tests were incubated for 24 hours.

RESULTS: 274 clinical Aspergillus isolates were tested, including A. fumigatus (73), A. flavus (51), A. niger (90), A. terreus (19), and A. nidulans (41). The Etest endpoint was lower than BMD for $91 \%$ of isolates with an overall essential agreement ( $\leq 2$ doubling dilution variance) of $47 \%$. The majority of discordant Etest results varied from BMD by 3 (33\%) and 4 (15\%) doubling dilutions, and was proportional across all species. Etest endpoints were clearly defined except for A. flavus and A. terreus, which tended to yield narrower inhibition zones with microcolony growth (disregarded).

CONCLUSIONS: Although susceptibility testing for filamentous fungi to the echinocandins is still in its infancy, the results of this study demonstrate that the caspofungin BMD MEC and Etest endpoint are not comparable measures. Unless CLSI test conditions can be optimized to reduce this disparity the caspofungin Etest cannot be used as a BMD alternative for Aspergillus susceptibility testing.

\section{Session D}

\section{D1}

\section{SENSITIVE AND SPECIFIC DETECTION OF A BROAD RANGE OF RESPIRATORY VIRUSES USING THE LUMINEX RVP ASSAY}

K Tokaryk ${ }^{1 *}$, K Pabbaraju ${ }^{1}$, S Wong ${ }^{1}$, J Fox ${ }^{1,2}$

${ }^{1}$ Provincial Laboratory for Public Health (ProvLab); ${ }^{2}$ Microbiology \& Infectious Diseases, University of Calgary, Calgary, AB

OBJECTIVE: The wide range of pathogens responsible for respiratory infections makes routine testing using individual real-time NATs expensive and laborious. The objective of this study was to compare the multiplex detection of 19 respiratory viral targets using the Luminex $\times \mathrm{Tag}^{\mathrm{TM}}$ Respiratory Viral Panel (RVP) with the individual real-time NATs used in ProvLab (Alberta).

METHODS: Nasopharyngeal samples were pre-screened by direct fluorescent antigen (DFA) for influenza (IFV) A and B, parainfluenza (PIV) 1-3 and respiratory syncytial virus (RSV). DFA positive samples were excluded from this comparative study. Respiratory specimens were collected between December 2006 and May 2007 with nucleic acid extraction undertaken using the easyMAG® extractor (bioMérieux). Individual 
real-time NATs for comparison with RVP were directed against IFVA, IFVB, PIV1-4, RSV, human metapneumovirus (hMPV) and respiratory adenoviruses. A total of 1497 specimens were part of the analysis with 632 specimens analyzed prospectively and 865 retrospectively by RVP. Positive specimens for coronaviruses or picornaviruses by the RVP assay were subsequently tested by real-time NATs as such testing is not part of our current diagnostic routine.

RESULTS: A total of 937 specimens were positive for one or more respiratory viruses by the individual NATs and 948 had one or more positive results by the RVP assay. The rate of detection for hMPV, IFVA, IFVB, PIV1-4 and RSV were comparable by both methods (approximately $3.0 \%$, $16.0 \%, 2.0 \%, 6.0 \%$ and $6.0 \%$ of total tested respectively). The detection of adenovirus was superior by the in-house NAT ( $4.1 \%$ positive compared with $2.4 \%$ by the RVP assay). However, the RVP assay identified $12.1 \%$ of samples as picornavirus positive (confirmed by sequencing), only half of which were identified by individual NATs undertaken for enteroviruses and rhinoviruses. Of the 248 IFVA positive specimens, $85.5 \%$ were subtyped as $\mathrm{H} 1$ or $\mathrm{H} 3$ by the RVP assay. A total of 26 samples positive for coronaviruses were detected by the Luminex RVP, which were all confirmed by real-time RT-PCR assays.

CONCLUSION: The Luminex RVP assay meets our current standards of sensitivity and specificity and also allows for multiplex detection of 19 respiratory viral targets with considerable time and cost savings compared with alternative NATs.

\section{D2}

\section{DETECTION OF NOROVIRUS, ROTAVIRUS AND} ADENOVIRUS IN STOOL SPECIMENS USING A NEW SENSITIVE AND SPECIFIC MULTIPLEX PCR

J Mahony*, S Chong

McMaster University, St. Joseph's Healthcare, Hamilton, ON

OBJECTIVE: Since most clinical laboratories use several methods including EM, culture, EIA and PCR to detect enteric viruses in stool specimens, we have developed a new multiplex PCR for the detection of Rotavirus, Norovirus and Adenovirus that could replace these older, insensitive methods.

METHODS: A multiplex PCR was designed to detect all known genotypes of Norovirus, Rotavirus and Adenovirus. Sequence alignment was performed, consensus sequences were identified for PCR, and degenerate primers were designed for all three viruses based on alignment of all known genotypes. Primers were designed for each viral target to generate small PCR products (155-238 bp) and evaluated using uniplex PCR for sensitivity and specificity using dilutions of known positive stools prior to multiplexing the primers and optimizing conditions for multiplex amplification. Detection of PCR products was performed by agarose gels and subsequently using micro-fluidic arrays. Nucleic acid extractions from stool specimens ( $140 \mathrm{uL}$ of $10 \%$ suspension) were performed using the MiniMag or EasyMag procedure and two step RT-PCR was performed using $5 \mathrm{uL}$ cDNA (neat or 1:10 dilution).

RESULTS: Norovirus, Rotavirus and Adenovirus PCR primers successfully detected Norovirus genogroups I and II including genotypes I.I, I.2 and I.3 and II.2, II.3, II.4, II.5, II.9 and II.13, Rotavirus types A, B and C, and Adenovirus by multiplex PCR respectively, in positive stools collected from outbreaks in Canada, the United States and China. Positive stools diluted between 10E-3 and 10E-6 were positive by multiplex PCR. Robust PCR products were detected on agarose gels with some non-specific products that disappeared when cDNA were diluted 1:10. Multiplex PCR was $100 \%$ sensitive for the detection of Norovirus (42/42), Rotavirus (33/33) and Adenovirus (3/3) respectively using a combined reference standard of EM, EIA or uniplex RT-PCR (Pang et al. 2004). False positive results have not been seen in testing 156 stool specimens.

CONCLUSION: We have developed a sensitive and specific multiplex PCR that can detect all known genotypes and serotypes of Norovirus, Rotavirus and Adenovirus. This assay could replace the traditional, insensitive tests currently used in clinical laboratories.

\section{D3}

EARLY IDENTIFICATION OF INFLUENZA A(H5) VIRUSES WITH AFFINITY FOR THE HUMAN SIALIC ACID RECEPTOR BY MALDI-TOF BASED MUTATION DETECTION

C Yea ${ }^{1}$, M Petric $^{3}$, R Tellier ${ }^{1,2 *}$

${ }^{1}$ Research Institute Hospital for Sick Children; ${ }^{2}$ Division of

Microbiology, Hospital for Sick Children, Toronto, ON; ${ }^{3}$ British

Columbia Centre for Diseases Control, Vancouver, BC

OBJECTIVE: The highly pathogenic strains of influenza A(H5N1) currently circulating have become the most dreaded candidate for giving rise to a pandemic. To become a pandemic strain, the $\mathrm{A}(\mathrm{H} 5 \mathrm{~N} 1)$ virus would have to switch sialic acid (SA) receptor affinity from the avian SA $\alpha 2,3 \mathrm{Gal}$ to the human SA $2,6 \mathrm{GAL}$. The required mutations are not known with certainty but likely mutations have been identified, based on subtypes H1, $\mathrm{H} 2$ and $\mathrm{H} 3$. Early identification of these mutations from field isolate would be advantageous.

METHODS: RT-PCR primers specific for A(H5) bracketing the regions of interest in the HA gene have been designed. Incorporation of a T7 core promoter in the primer allows for in vitro transcription and translation, followed by MALDI-TOF mass spectrometry analysis. This method will permit identification of the candidate mutations for receptor affinity switch even if present in a quasispecies.

RESULTS: A full length cDNA for the HA encoding RNA segment of A/Vietnam/2004 (H5N1) was cloned into a plasmid (pGEM-7Zf+, Promega). By site directed mutagenesis we created HA mutants with the mutations Gln226Leu, Gly228Ser, and with both mutations. Full length negative strand RNAs transcribed from the plasmids were used in the RTPCR, translated in vitro and submitted to MALDI-TOF mass spectrometry. Peptides encoded by the wild type and mutated HA genes could be distinguished by MALDI-TOF

CONCLUSION: Analysis of the expressed HA proteins by MALDI-TOF can readily distinguish mutated forms with altered affinity to the sialic acid receptor. This approach is particularly important if the maturated HA exists as a quasispecies, whose presence is more readily detectable by MALDI-TOF than be genomic sequence analysis.

\section{D4}

\section{DEVELOPMENT OF A NUCLEIC ACID AMPLIFICATION TEST ON THE LUMINEX PLATFORM FOR BROAD DETECTION OF ARBOVIRUSES}

K Pabbaraju ${ }^{1 *}$, S Wong1 ${ }^{1}, \mathrm{~K} \mathrm{Ho}^{1}$, KL Tokaryk ${ }^{1}$, JD Fox ${ }^{1,2}$, PAG Tilley ${ }^{1}$ Provincial Laboratory for Public Health; ${ }^{2}$ University of Calgary, Calgary, AB

OBJECTIVE: Changing global environmental conditions and increased transport of people and goods threaten to introduce new viruses into naïve unexposed populations. In particular the extension of arboviruses to new geographic regions as in the cases of Japanese encephalitis virus (JEV) and West Nile virus (WNV) are causes for concern. We have developed a Luminex suspension array based assay using primer pairs targeting conserved genomic regions for detection of a broad-range of arboviruses. In addition we have also developed species-specific assays for the detection of Western equine encephalitis virus (WEE), Eastern equine encephalitis virus (EEE), WNV and enteroviruses.

METHODS: Arbovirus controls belonging to the Flaviviridae, Bunyaviridae and Alphaviridae families were obtained from the National Microbiology Laboratory (Winnipeg, Manitoba, Canada). A two-step RTPCR protocol was developed using six sets of primers for amplification of arboviruses and enteroviruses. Eleven amino labelled probes were coupled to carboxy-microsphere beads for the detection of amplified products using suspension arrays and flow cytometry on a Luminex xMAP® platform.

RESULTS: All the control viruses including St. Louis encephalitis virus (Parton), Powassan virus (M51725), dengue viruses 1-4 ( Hawaii, New Guinea, H87, and H241), WNV (NY99), snowshoe hare viruses (942, 71-Y-23, WNL, 25-A3-A4, 80-Y-160, and 74-Y-235), EEE (QA80), WEE (QMcMillan), Jamestown Canyon virus (74-31), and enterovirus (echo 2) could be amplified successfully using the RT-PCR protocol and detected by the Luminex suspension array. Plasma and CSF specimens positive for 
WNV and enterovirus, and mosquito pools positive for WNV and Cache Valley virus could be detected by the developed assay. This assay will be further validated on CSF and plasma specimens from patients and implemented for the detection of a broad range of arboviruses and enteroviruses. CONCLUSION: Broadly reactive molecular methods have the potential to screen rapidly for both known and unknown viral species. The suspension array-based assay described can be used for the simultaneous detection of a wide-range of arboviruses. It is fast, easy to perform and high throughput in nature and will be invaluable for surveillance and ultimately, routine diagnostic applications.

\section{D5}

COMPARISON OF THE ROCHE COBAS AMPLIPREP/COBAS TAQMAN $^{\mathrm{TM}}$ HCV AND THE VERSANT ${ }^{\mathrm{TM}}$ HCV RNA 3.0 TESTS FOR MEASUREMENT OF HEPATITIS C VIRUS VIRAL LOAD W Mei ${ }^{3 *}$, D Cook ${ }^{1}$, A Mak ${ }^{3}$, K Gunadasa ${ }^{3}$, B Leung ${ }^{3}$, M Krajden ${ }^{1,2}$ ${ }^{1}$ British Columbia Centre for Disease Control; ${ }^{2}$ University of British Columbia; ${ }^{3}$ PHSA Laboratories, Vancouver BC

OBJECTIVE: A virological cure for Hepatitis C Virus (HCV) can now be achieved in approximately $55 \%$ of patients treated with pegylated interferon and ribavirin. Failure to achieve a 100 -fold or a $2-\log _{10}$ reduction in viral load within $12 \mathrm{wks}$ of treatment is predictive of treatment failure and residual viraemia at wk 12 is also a marker for poor response. The performance of the Roche COBAS AmpliPrep/COBAS TaqMan ${ }^{\mathrm{TM}} \mathrm{HCV}$ (CAP/CTM) was compared with the Versant ${ }^{\mathrm{TM}}$ HCV RNA 3.0 (bDNA) (V-bDNA) assay for measuring HCV viral loads in patients undergoing therapy.

METHODS: One hundred nine (109) specimens with a range of viral loads were tested by both methods. The correlation coefficient $\left(\mathrm{R}^{2}\right)$ between the two tests and the mean $\log _{10}$ difference were calculated.

RESULTS: Seventy-six (76) specimens had measurable viral loads by both assays. For these specimens, the $\mathrm{R}^{2}$ was 0.86 and the viral loads measured by CAP/CTM were an average of $0.28 \log _{10}$ higher than those by V-bDNA. Twenty (20) specimens had undetectable viral loads by both assays. Twelve (12) specimens had undetectable viral load by V-bDNA (i.e. $<615 \mathrm{IU} / \mathrm{mL}$ ), but had measurable viral loads by CAP/CTM (range $<15$ to $2000 \mathrm{IU} / \mathrm{mL}$ ). One (1) specimen had a viral load of $2173 \mathrm{IU} / \mathrm{mL}$ by V-bDNA but no target RNA was detected by CAP/CTM.

CONCLUSIONS: The correlation coefficient between the CAP/CTM and V-bDNA was 0.86 . The advantages of the CAP/CTM assay include a wide dynamic range and a lower limit of detection (i.e., $15 \mathrm{IU} / \mathrm{mL}$ or approx. 50- to 100-fold more sensitive than that of the V-bDNA), which is clinically relevant based on the data supporting residual viraemia at wk 12 correlating with poor clinical outcome. However, CAP/CTM viral loads were $0.28 \log _{10}$ higher than those measured by V-bDNA which suggests that individual patients should undergo serial testing using the same assay to monitor therapeutic response.

\section{D6}

\section{LABORATORY DIAGNOSIS OF MUMPS IN A PARTIALLY IMMUNIZED POPULATION}

T Hatchette ${ }^{1,2 *}$, R Davidson ${ }^{1,3}$, S Clay ${ }^{5}$, J Pettipas ${ }^{1}$, J Leblanc ${ }^{1}$, S Sarwal ${ }^{6}$, G Watson Creed ${ }^{4,7}$, K Forward $^{1,2}$

${ }^{1}$ Department of Pathology and Laboratory Medicine, QEII HSC; ${ }^{2}$ Department of Pathology, Dalhousie University; ${ }^{3}$ Department of Microbiology and Immunology, Dalhousie University; ${ }^{4}$ Community Health and Epidemiology, Dalhousie University, Halifax, NS; ${ }^{5}$ Canadian Field Epidemiology Program, Public Health Agency of Canada, Ottawa, ON; ${ }^{6}$ Nova Scotia Department of Health; ${ }^{7}$ Nova Scotia Department of Health Promotion and Protection, Halifax, NS OBJECTIVE: This year in Atlantic Canada we saw a large outbreak of mumps predominately in university students who had received a single dose of MMR vaccine. The objective of this study is to present the performance characteristics of RT-PCR on oral and urine specimens and IgM serology in this partially immune population.

METHODS: Patients presenting with symptoms suspicious for mumps had a serum, urine and an oral swab collected and sent for diagnostic testing. Persons were classified as a "confirmed" case if positive for either mumps IgM or mumps virus based upon PCR or in the absence of a positive laboratory test if they presented with bilateral parotitis or unilateral parotitis with a confirmed epidemiologic link. Sera were tested using Enzygnost IgM enzyme linked immunoassay (Dade Behring). Detection of mumps virus in oral and urine samples was by reverse transcriptase PCR (RT-PCR) using a hemi-nested protocol previously described (Jin et al., 1999).

RESULTS: Between February and June 2007 we performed 3410 RT-PCR specimens from 2082 patients and IgM testing on 1460 sera from 1359 patients. A subset of 158 cases and 374 non-cases had all three specimens submitted as a set were used for calculating the performance characteristics outlined below. Only 12/134 patients had positive urine specimens in the presence of negative oral swabs.

\begin{tabular}{lccccc} 
& $\begin{array}{c}\text { Sensitivity } \\
\text { (\%) }\end{array}$ & $\begin{array}{c}\text { Specificity } \\
\text { (\%) }\end{array}$ & $\begin{array}{c}\text { PPV } \\
\text { (\%) }\end{array}$ & $\begin{array}{c}\text { NPV } \\
\text { (\%) }\end{array}$ & $\begin{array}{c}\text { Accuracy } \\
\text { (\%) }\end{array}$ \\
\hline Oral Swab & 78.3 & 99.5 & 98.4 & 91.6 & 93.2 \\
Urine & 42.4 & 100 & 100 & 80.4 & 82.9 \\
IgM serology & 25.0 & 99.7 & 97.5 & 75.8 & 77.4 \\
\hline
\end{tabular}

CONCLUSION: We show that RT-PCR on oral swabs is the ideal specimen for diagnosis and that testing an additional urine sample did not significantly increase the diagnostic yield significantly but doubled the cost of testing. In addition, it is clear from our data that in this partially immune group, IgM serology has little value in the diagnosis of acute infection.

\section{Session E}

\section{E1} OVINE-ASSOCIATED Q FEVER IN NOVA SCOTIA; CASE
REPORT AND SHEEP SEROPREVALENCE STUDY D Webster ${ }^{*}$, D Haase ${ }^{2}$, TJ Marrie ${ }^{3}$, N Campbell ${ }^{2}$, J Pettipas ${ }^{2}$, R Davidson², T Hatchette ${ }^{2}$

${ }^{1}$ Saint John Regional Hospital, Saint John, NB; ${ }^{2}$ Dalhousie University, Halifax, NS; ${ }^{3}$ University of Alberta, Edmonton, AB OBJECTIVE: In Atlantic Canada, the major risk factor for acquisition of $Q$ fever infection has been identified as exposure to infected parturient cats or new-born kittens. In this study, we describe, the first case of $Q$ fever in Nova Scotia, acquired as a result of direct exposure to sheep and undertake a serosurvey of the associated flock.

METHODS: A serosurvey of the sheep flock, associated with the clinical case, was performed using blood samples collected from 46 sheep. Testing for anti-phase I and anti-phase II C. burnetii antibodies was performed by indirect immunofluorescence (IFA). Fresh milk was also obtained from four nursing ewes and DNA amplifcation using polymerase chain reaction (PCR) was employed to detect C. burnetii in the sheep milk specimens.

RESULTS: The sheep serosurvey revealed that 23 of 46 sheep (50\%) were seropositive for the anti-phase II antibody. A single ewe of the 46 sheep (2.2\%) demonstrated anti-phase I antibody seropositivity and this ewe also had the study's highest anti-phase II antibody titer (1:128). Four of 46 sheep $(8.7 \%)$ had anti-phase II antibody titers of 1:64. This group of four included three nursing ewes, one of which had delivered two lambs that died shortly after delivery. Seven of 46 sheep (15.2\%) had anti-phase II antibody titers of 1:32. Four of these seven were lambs that were nursing. However, molecular studies failed to detect C. burnetii DNA in any of the milk specimens obtained from these four ewes.

CONCLUSIONS: We describe the first case of Q fever in Nova Scotia, acquired through direct exposure to sheep. The follow-up sheep seroprevalence study demonstrates that C. burnetii has now spread to a more traditional animal reservoir in Nova Scotia. 
E2

\section{A REVIEW OF MOLECULAR TYPING OF TB ISOLATES IN ALBERTA}

L Chui $^{2 *}$, R Long ${ }^{1}$, G Tyrrell ${ }^{2}$, J Preiksaitis ${ }^{2}$, D Kunimoto ${ }^{1}$

${ }^{1}$ University of Alberta, Edmonton, AB; ${ }^{2}$ Provincial Laboratory for

Public Health, Edmonton, AB

OBJECTIVE: Molecular typing in generating DNA fingerprints for strain differentiation plays a major role in the study of epidemiology in infectious diseases. This tool is critically important as an aid to identify the source of potential outbreaks and for identifying the extent of the spread of clones causing disease. The objective of this report is to describe the TB typing database housed in the Provincial Laboratory for Public Health (Provlab). MATERIALS AND METHODS: All M. tuberculosis isolates in Alberta have identification and susceptibility testing performed in the Provlab. The first isolate grown from a patient has molecular typing by RFLP. IS6110 hybridization by a standard protocol. Isolates that showed less than 6 bands in the IS6110 fingerprinting profile were subjected to spoligotyping as per manufacturer's instruction. All DNA fingerprints generated by IS6110 RFLP and spoligotyping were entered into the BioNumerics Provlab database for analysis.

RESULT: The Provlab in Alberta possess a large collection of 1777 IS6110 DNA fingerprints from clinical TB isolates from the latter half of 1990 to December, 2006. Spoligotyping was performed on all 221 isolates with a low copy number of IS6110 elements. In total, using both IS6110 and spoligotyping, 1122 fingerprint patterns were unique. 170 clusters were identified involving 655 isolates ranging from 2 to 36 isolates per cluster. This database has proven useful in 1) contact tracing 2) identifying the possible cross contamination in the laboratory and 3) in population based analysis of TB in Alberta.

CONCLUSION: The Provlab in Alberta now possess a large TB database consisting of TB isolates DNA typed by IS6110 analysis and where necessary, spoligotyping. This database consists of 1777 isolates and contains 170 clusters ranging in size from 2 to 36 isolates (655 isolates in total). There are currently 1122 TB isolates in this database that do not have a matching DNA fingerpint in Alberta. This database has proven useful as a tool in the aid of the epidmiological investigation of known or suspect TB cases in Alberta.

\section{E3}

\section{SEASONAL VARIATION AND ENVIRONMENTAL EFFECTS IN INVASIVE MENINGOCOCCAL DISEASE IN PHILADELPHIA, PENNSYLVANIA}

L Kinlin ${ }^{1}$, V Ng-Brett ${ }^{1}$, V Spain ${ }^{3}$, C Johnson ${ }^{3}$, A White ${ }^{1}$, D Fisman ${ }^{1,2}$

${ }^{1}$ Hospital for Sick Children; ${ }^{2}$ Ontario Central Public Health Laboratory, Toronto, ON; ${ }^{3}$ Philadelphia Department of Public Health, Philadelphia, Pennsylvania, United States

OBJECTIVE: Invasive meningococcal disease (IMD) is an important cause of meningitis, bacteremia, and associated mortality worldwide. Seasonal variation in its incidence has long been recognized but mechanisms responsible for this phenomenon remain poorly understood. In light of recent concerns related to global climate change, we sought to evaluate the effect of environmental factors on IMD risk in a major urban centre.

METHODS: The association between monthly weather patterns and IMD incidence in Philadelphia, Pennsylvania was evaluated using Poisson regression analysis. Multivariable regression models included monthly and yearly terms to account for underlying seasonal and temporal trends. Acute (i.e., short-term) weather effects important in disease occurrence were identified through a case-crossover approach. Both study designs controlled for seasonal factors that might otherwise confound the relationship between environment and IMD.

RESULTS: Public health authorities in Philadelphia received 162 reports of IMD occurring between 1995 and 2006. Incidence, which was highest in the winter and spring, displayed a significant seasonal pattern ( $P$ for seasonal oscillation $<0.001$ ). Poisson regression, including seasonal and yearly smoothers, elevated risk with increasing relative humidity (IRR per $1 \%$ increase in maximum relative humidity, 1.04 [95\% CI, 1.0041.08]). Using case-crossover methods, an acute protective association was found for clear sky UV index during the period 1-4 days prior to onset of cases (OR, 0.55 [95\% CI, 0.36-0.84]). Further analysis revealed a significant negative dose-response pattern between this meteorological variable and case occurrence (Wald chi-squared for trend, $4.22[1 \mathrm{df}]$; $\mathrm{P}=0.04$ ).

CONCLUSIONS: In contrast to the association with "dry season" in the sub-Saharan meningitis belt, extended periods of high humidity are an important predictor of IMD occurrence in Philadelphia, and UV radiation appears to protect against disease, possibly through a mechanism of decreased pathogen survival or virulence. These findings underscore the importance of environmental factors in infectious disease occurrence and may serve to explain the seasonality of IMD incidence.

\section{E4}

\section{CHARACTERIZATION OF STREPTOCOCCUS PYOGENES AND STAPHYLOCOCCUS AUREUS ISOLATES ASSOCIATED WITH MIXED SKIN AND SOFT TISSUE INFECTIONS IN NORTHERN SASKATCHEWAN}

RR McDonald ${ }^{1 *}$, GR Golding ${ }^{2}$, PN Levett ${ }^{1}$, CK Yost $^{3}$, J Irvine ${ }^{4}$, GB Horsman ${ }^{1}$, MR Mulvey ${ }^{2}$, the Northern Antibiotic Resistance Partnership (NARP)

${ }^{1}$ Saskatchewan Disease Control Laboratory, Saskatchewan Health, Regina, SK; ${ }^{2}$ National Microbiology Laboratory, Public Health Agency of Canada, Winnipeg, MB; ${ }^{3}$ Department of Biology, University of Regina, Regina, SK; ${ }^{4}$ Athabasca Health Authority, Keewatin Yatthe Health Region, and Mamawetan Churchill River Health Region, La Ronge, SK

OBJECTIVES: Community-associated infections are a significant public health concern worldwide. In particular, antibiotic-resistant pathogenic bacteria associated with skin and soft tissue infections (SSTI) are becoming more prevalent in certain populations. Through an ongoing surveillance program, a high number of SSTI specimens yielding S. pyogenes along with S. aureus have been identified. The objective of this study is to characterize $S$. pyogenes and S. aureus isolates associated with mixed SSTI infections by molecular typing and antibiotic resistance profiles.

METHODS: Between Oct. 2005 and Jan. 2007, S. pyogenes and S. aureus isolates were collected from skin and soft tissue infections from three northern Saskatchewan communities. Methicillin resistance was determined in S. aureus by resistance to oxacillin and by PCR for mecA, nuc, and PVL-encoding genes. S. pyogenes isolates were subtyped by emm sequencing and $S$. aureus were subtyped by spa-typing and pulsed-field gel electrophoresis (PFGE). The minimum inhibitory concentration (MIC) of 27 antibiotics was determined by broth microdilution susceptibility testing according to CLSI guidelines.

RESULTS: 175 S. pyogenes, 248 methicillin-resistant S. aureus (MRSA) and 229 methicillin-sensitive $S$. aureus (MSSA) were isolated from SSTI. 119 specimens indicated mixed infections involving $S$. pyogenes and $S$. aureus. Of these, $S$. pyogenes was more commonly associated with MSSA $(\mathrm{n}=98)$ than with MRSA $(\mathrm{n}=21, \mathrm{p}<0.0001) .20$ unique emm types were identified, with type 41.2 being the most common $(n=28)$. The most common of the 27 MSSA spa-types was $\mathrm{t} 311 \quad(\mathrm{n}=20)$ and $90.5 \%(19 / 21)$ of MRSA were PFGE epidemic type CMRSA7. Ten specimens yielded S. pyogenes emm-type 41.2 with MSSA spa-type $\mathrm{t} 311$ as the most frequent combination observed. With the exception of fusidic acid $\left(\mathrm{MIC}_{50}=8 \mathrm{mg} / \mathrm{L}\right)$, S. pyogenes isolates were susceptible to all antibiotics tested.

CONCLUSIONS: Both S. pyogenes and MSSA associated with co-infections have diverse genetic backgrounds, while most MRSA represent the CA-MRSA clone, CMRSA7, found to be common in this geographic region. Despite circulation of antibiotic-resistant S. aureus, S. pyogenes remains susceptible to many antibiotics. Future work is required to determine virulence of and interactions between S. pyogenes and S. aureus in these cases. 


\section{E5}

\section{SURVEILLANCE SYSTEMS FOR EMERGING INFECTIOUS DISEASES: DEFINITIONS OF SURVEILLANCE AND EVALUATION}

L Vrbova $^{1 *}$, C Stephen ${ }^{1,2,3}$, N Kasman ${ }^{4}$, R Boehnke ${ }^{6}$, M Doyle-Waters ${ }^{5}$, B Gibson ${ }^{6}$, A Chablitt-Clark ${ }^{6}$, M Brauer ${ }^{1}$, M Fitzgerald ${ }^{1,5,7}$, D Patrick,

${ }^{1}$ University of British Columbia, Vancouver, BC; ${ }^{2}$ University of Calgary, Calgary, AB; ${ }^{3}$ Centre for Coastal Health, Nanaimo, BC; ${ }^{4}$ York Region Health Services Dept, Newmarket, ON; ${ }^{5}$ Vancouver Coastal Health Research Institute, Vancouver, BC; ${ }^{6}$ Ontario Ministry of Health and Long-Term Care, Toronto, $\mathrm{ON}$; ${ }^{7}$ Vancouver General Hospita; ${ }^{8}$ British Columbia Centre for Disease Control, Vancouver, BC

BACKGROUND: EID surveillance systems are proliferating. There is a need to examine whether these systems are being developed and evaluated using evidence-based methods in order to properly interpret their early warning value and to recommend accountable allocation of resources for development and support. The purpose of this study was to conduct a systematic review on surveillance systems for EIDs and their evaluation. METHODS: A broad search was employed to search peer-reviewed literature published worldwide 1992-2006 that described and/or evaluated EID surveillance systems, focusing on zoonotic EIDs. Grey literature, consisting of surveillance system evaluations conducted in Canada, was obtained from public health practitioners. Preliminary results of the review were discussed by all authors at a one-day meeting intended to develop consensus on sources of information, inclusion/exclusion criteria, data extraction, and plan of analysis.

RESULTS: 209 articles were included in the review describing 225 emerging zoonosis surveillance systems. Many systems that were described as surveillance systems were misclassified as they did not include any timesensitive response component, and were therefore either case detection systems or monitoring systems. More than half of the articles were published in the last quarter of the study (2003-6), suggesting many of the systems are still new. Only 17 of the 225 systems were evaluated, and only four of these used the evaluation results to support claims that their systems were useful or not useful in identifying outbreaks or cases of disease. Although the grey literature of surveillance system evaluations was more informative than the peer-reviewed literature, it was initially difficult to locate the articles.

CONCLUSIONS: This systematic review has shown that many systems that claim to be surveillance systems for emerging zoonoses are in fact only monitoring or case detection systems. Further, less than $10 \%$ of the systems have been evaluated, suggesting that they have not been developed using evidence-based methods. This lack of readily available information makes it difficult for decision-makers to choose surveillance initiatives that have been proven effective.

\section{E6}

RE-EMERGENCE OF PATHOGENIC VIBRIO VULNIFICUS ON THE PACIFIC NORTHWEST COAST IN 2007

M Bigham ${ }^{1,3 *}$, E Galanis ${ }^{1,2}$, R Gustafson $^{1,4}$, L Hoang ${ }^{1,2}$, K $^{2}$ Louie $^{3}$, A Faremo $^{4}$, L Mcintyre ${ }^{2}$, J Isaac-Renton ${ }^{1,2}$, P Daly ${ }^{1,4}$, L Gustafson ${ }^{3}$, B Wong' ${ }^{2}$, S Chong ${ }^{4}$, J Stone ${ }^{3}$, L Rodriguez-Maynez ${ }^{5}$

${ }^{1}$ University of British Columbia; ${ }^{2}$ British Columbia Centre for Disease Control, Vancouver; ${ }^{3}$ Fraser Health Authority, Surrey; ${ }^{4}$ Vancouver Coastal Health Authority; ${ }^{5}$ Canadian Food Inspection Agency, Vancouver, BC

OBJECTIVES: Vibrio vulnificus is a naturally occurring marine pathogen previously reported in oysters recovered from $\mathrm{BC}$ coastal waters during summer months. Human infection, rarely recognized in the Pacific Northwest, may result from either consuming contaminated shellfish or from environmental exposure, and can cause severe disease. Over a 2 week period in mid-July 2007, two Vancouver BC area residents developed severe $V$. vulnificus disease, not associated with shellfish consumption. This report summarizes the public health investigation and response to these cases.
METHODS: An epidemiologic investigation of cases was carried out. Environmental sampling and testing were undertaken, including microbiologic analysis of water collected from local beach tidal pools and sediment. A risk assessment guided public health action.

RESULTS: The cases were a 54 year old previously healthy male with severe cellulitis of the lower left leg and septicemia, and a 79 year old immunosuppressed female presenting with necrotizing fasciitis of the lower right leg. The only identified risk for $V$. vulnificus exposure in both cases was wading along two area beaches several days prior to onset of illness. $V$. vulnificus was recovered from water samples from three sites along beach "A". V. parahemolyticus was the only Vibrionaceae recovered from beach "B", although recent log dredging near beach "B" may have transiently released other undetected Vibrio species from disturbed sediment. Local marine water temperatures were within normal historic seasonal ranges. Beach "A" was temporarily closed during the investigation.

CONCLUSIONS: In both cases, V. vulnificus infection likely resulted from exposure through an unrecognized skin break to contaminated water while wading in shallow water at two Vancouver area beaches. V. vulnificus, naturally present in Pacific Northwest marine waters, can proliferate in summer and pose a potential human health hazard. Risk assessment concluded that existing public health messaging, (proper handling/cooking of shellfish; preventing exposure of open wounds to recreational waters) was adequate.

\section{Session $F$}

\section{F1}

\section{EVALUATION OF THE ESWAB FOR MRSA SCREENING} WITH REAL TIME PCR, BD GENEOHM AND CULTURE

\section{S Castriciano*, A Giambra}

Copan Italia, Brescia, Italy

BACKGROUND: Collection, transportation and preservation of clinical specimens for screening patients for MRSA colonization is usually done in Stuart liquid medium or in Amies gel transport system. Copan Diagnostic (Brescia, Italy) has released the ESwab, a new collection and preservation device with a flocked swab and a screw cap tube containing one $\mathrm{ml}$ of liquid Amies medium. After the specimen is collected, the flocked swab is broken into the tube with liquid Amies: the sampled material is instantly released by the flocked swab into the liquid, resulting in a homogeneous specimen suspension.

OBJECTIVE: The objective was to compare the ESwab and Stuart liquid transystem sensitivity using Real Time PCR, BD GeneOhm Assay (GO) and culture for the detection of MRSA at different storage times and temperatures.

METHODS: One MRSA ATCC strain, 3 clinical MRSA strains and samples from volunteers were used in this study; all samples were tested by PCR, BD GeneOhm Assay and chromogenic agar (BioRad). Serial dilutions of the ATCC strain were inoculated in ESwab and Stewart liquid Transystem, and tested at $\mathrm{t}=0$ and after $24 \mathrm{~h}$ storage at room temperature (RT). ES and ST, inoculated respectively with an ATCC and 3 clinical MRSA strains at $\sim 200 \mathrm{CFU}$, were tested at $\mathrm{t}=0$ and after $24 \mathrm{~h}$ at RT, and 3 months at $-20^{\circ} \mathrm{C}$. Each volunteer $(n=10)$, was swabbed in separate nostril with ES and ST. The impact of clinical samples, for both devices, was evaluated on the GO internal control.

RESULTS: After 24h, the detection limit for the ATTC strain with real time PCR and GeneOhm Assay was $10^{-5}$ for ES and $10^{-4}$ for ST samples. Culture detected all MRSA strains at all storage times. After 3 month's storage: real time PCR detected 2/4 positive from ST and 3/4 positive from ES; GO detected 3/4 positive from ST and 4/4 from ES. The volunteer's samples were all negative: the GeneOhm Assay had two unresolved from each device that were solved after freezing and thawing the specimens. CONCLUSIONS: The Copan ESwab improved the sensitivity of all methods and is compatible with PCR and BD GeneOhm Assay. ESwab provides, from a single sample-collection, a ready-to-test homogeneous suspension of the original specimen, suitable for multiple testing methods and long-term storage. 


\section{F2}

A GUIDELINE FOR THE MOLECULAR TYPING OF CANADIAN EPIDEMIC METHICILLIN-RESISTANT STAPHYLOCOCCUS AUREUS USING SPA TYPING GR Golding ${ }^{1}$, JL Campbell ${ }^{1}$, DJ Spreitzer ${ }^{1}$, J Vehyl ${ }^{2}$, K Surynicz ${ }^{2}$, A Simor $^{3}$, MR Mulvey ${ }^{1}$

${ }^{1}$ National Microbiology Laboratory; ${ }^{2}$ University of Manitoba, Winnipeg, MB; ${ }^{3}$ Sunnybrook Health Sciences Centre, Toronto, ON OBJECTIVE: Increasing incidences of methicillin-resistant Staphylococcus aureus (MRSA) infections have resulted in the overburden of many Canadian laboratories with technically demanding typing methods such as pulsed-field gel electrophoresis (PFGE) and multi-locus sequence typing. The purpose of this study was to determine if spa typing could be used as an alternative to PFGE for the identification of Canadian epidemic MRSA (CMRSA) isolates.

METHODS: A total of 1494 CMRSA isolates, representing 807 unique PFGE types, were selected from a twelve year collection (1995-2006) of over 15,000 MRSA isolates obtained through the Canadian Nosocomial Infection Surveillance Program (CNISP). Isolates were initially chosen on the basis of unique PFGE patterns that were associated with one of the 10 epidemic CMRSA strains. When possible, multiple isolates of the same epidemic PFGE pattern were chosen by varying geographical distribution and/or time of isolation. DNA extracted from glass bead disruption media was used as the template for PCR amplification of the spa repeat region. DNA sequences were subsequently analyzed using the BioNumerics $\mathrm{v} 4.61$ spa plug in tool.

RESULTS: In this study, 116 unique spa types were identified from the 1494 tested CMRSA isolates. Minimal spanning tree analysis of the spa types revealed separate clonal clusters for PFGE epidemic types CMRSA1, 2, 7, and 8, but could not distinguish CMRSA5 from CMRSA9 and CMRSA10, and CMRSA3 from CMRSA4 and CMRSA6. Despite the inability to clearly differentiate all PFGE epidemic types using spa clustering, the majority of individual spa types within these clusters (112/116) were only associated with one PFGE epidemic type. Based on these results we developed an algorithm to be used as a guideline for spa typing of CMRSA isolates. This algorithm was used to prospectively type the first 300 MRSA isolates received in 2007 through the CNISP study, which correctly predicted the epidemicity of the strains as compared to PFGE.

CONCLUSION: The high concordance of spa typing with PFGE epidemic types using this algorithm demonstrated the feasibility of spa typing as a more rapid, less technically demanding, and potentially cheaper alternative typing method for MRSA in Canada.

\section{F3}

PREVALENCE OF INVASIVE METHICILLIN RESISTANT STAPHYLOCOCCUS AUREUS (MRSA) WITH REDUCED SUSCEPTIBILITY OR TOLERANCE TO VANCOMYCIN (VA) IN 2006 FROM FOUR HOSPITAL SYSTEMS IN ONTARIO: HAMILTON HEALTH SCIENCES (HHS), ST. JOSEPH'S HEALTHCARE (STJ), WILLIAM OSLER (WO) AND NIAGARA HEALTH SYSTEM (NHS).

D Yamamura ${ }^{1,2 *}$, L Wilcox ${ }^{2}$, C Rutherford ${ }^{2}$, P Lyn $^{2}$, D Richardson ${ }^{4}$, C Main ${ }^{1,2}$, S Dunnett ${ }^{5}$, N Morgan ${ }^{1}$, L Van Zuilen ${ }^{3}$, G Ricci ${ }^{4,2}$, CH Lee ${ }^{1,2}$

${ }^{1}$ McMaster University; ${ }^{2}$ Hamilton Regional Laboratory Medicine Program, Hamilton; ${ }^{3}$ LifeLabs, Thorold; ${ }^{4}$ William Osler Health Center, Brampton; ${ }^{5}$ Niagara Health System, St. Catharines, ON OBJECTIVE: Invasive MRSA cause significant morbidity and mortality. VA is commonly used for treatment; however, high level and intermediate VA resistance have been reported. Treatment failure can also occur due to heterogeneous VISA (hVISA) which are difficult to detect by routine methods. The objective was to determine the prevalence of invasive MRSA with reduced susceptibility or tolerance to VA.

METHODS: Consecutive invasive MRSA from HHS, StJ, WO and the NHS were evaluated. Isolates were identified by gram, catalase, Pastorex and tube coagulase. MICs were performed by broth microdilution (BMD) GPN3F (TREK) panels. VA E-test (ET) was performed on MHA and BHI with inocula equivalent to 0.5 and $2.0 \mathrm{McFarland}$ and read at 24/48h. Minimum bactericidal concentration (MBC) was performed using BMD with isolates in log-phase growth. MBC was calculated using $99.9 \%$ endpoint at 24/48h. QC strains E. faecalis ATCC 29212, S. aureus ATCC 29213, and ATCC 700699 mu3 S. aureus were included. PCR for mecA, nuclease, and PVL was performed as previously described. Chart review was by standardized form.

RESULTS: 58 MRSA isolates were evaluated. In patients from HHS/WO $(n=27), 48 \%$ were colonized with MRSA prior to the invasive isolate; none had VRE; 24 were health-care and 3 were community associated. MICs of VA were method dependent:

\begin{tabular}{lccccc} 
VA MIC & BMD & ET 0.5 MH & ET 2.0 MH & ET 0.5 BHI & ET 2.0 BHI \\
\hline$\leq 1$ & 56 & 45 & 35 & 0 & 0 \\
2 & 2 & 13 & 23 & 44 & 28 \\
4 & 0 & 0 & 0 & 14 & 29 \\
\hline
\end{tabular}

All isolates were susceptible to linezolid, daptomycin, quinupristin-dalfopristin, TMP-SMX. VA MBCs ( $\mathrm{n}=52)$ were $\leq 4(62 \%), 8-16(25 \%), \geq 32$ (13\%) and clinical cure/death rates were $71 \% / 29 \%, 33 \% / 56 \%$, and $50 \% / 50 \%$ respectively $(\mathrm{n}=27$ patients). Seven isolates displayed tolerance (MBC:MIC $\geq 32$ ). BMD/ET did not differentiate isolates with increase VA $\mathrm{MBC}$ or tolerance.

CONCLUSIONS: Reduced susceptibility to VA is uncommon in invasive MRSA isolates. Tolerance to VA was detected in $13 \%$ of isolates. Elevated $\mathrm{MBC} /$ tolerance may be associated with increased treatment failure. Further investigation with a larger population and modified population analysis to detect hVISA is warranted.

\section{F4}

\section{METHICILLIN-RESISTANT STAPHYLOCOCCUS AUREUS (MRSA) ENDOCARDITIS AND DAPTOMYCIN RESISTANCE DE NOVO DEVELOPMENT DURING THERAPY}

L Twele ${ }^{1,2 *}$, E Moyen ${ }^{1,2}$, J Kim ${ }^{1,2}$, B Dalton ${ }^{2}$, K Zhang1,2,3, D Church ${ }^{1,2,3}$, J Conly $y^{1,2,3}$

${ }^{1}$ University of Calgary; ${ }^{2}$ Calgary Health Region; ${ }^{3}$ Calgary

Laboratory Services, Calgary, AB

OBJECTIVES: Daptomycin resistance in Staphylococcus aureus (SA) has been reported previously but the development of resistance while on therapy with subsequent clinical failure during treatment for endocarditis has been reported only once. We present a case of persistent MRSA bacteremia in the setting of right-sided endocarditis with de novo resistance development to daptomycin during therapy with subsequent clinical failure.

METHODS: A 38 year old male with a history of poly-substance abuse and Hepatitis $\mathrm{C}$ was diagnosed with MRSA empyema, pneumonitis and endocarditis. Persistent bacteremia occurred despite multiple antibiotics including vancomycin, rifampin, linezolid, tigecycline and cefepime. Despite the use of daptomycin $12 \mathrm{mg} / \mathrm{kg}$, to which the isolate was sensitive, the bacteremia persisted until a combination of gentamicin, linezolid and ertapenem was initiated. Clinical, pharmacy and laboratory data were collected by review of relevant medical records. Isolates were collected from day 1 (pre-treatment), 58 and 64 (during daptomycin treatment) and submitted for detailed molecular characterization. MRSA was identified using standard laboratory procedures and confirmed by PCR assay for nuc, femA and mecA. MICs were determined by E testing. Molecular characterization was done with pulsed-field electrophoresis (PGFE), SCCmec, spa and Multi Locus Sequence Typing (MLST) and PCR assay for Panton Valentine leukocidin (PVL) and ACME.

RESULTS: Clinical review indicated the patient had tricuspid valve endocarditis with annular abscess. With a MIC of $0.19 \mathrm{mg} / \mathrm{l}$ to the MRSA, daptomycin was started on day 52 and was discontinued when the MIC was $1.5 \mathrm{mg} / \mathrm{l}$ on retesting with persistent bacteremia. Molecular testing and PGFE analysis revealed all three isolates were identical and were CAMRSA10 (USA300) with mecA+, PVL+, ACME+, $\phi S a 2 u s a$ phage+, MLST type ST8 but spa type t024 and carried SCCmec type IVa.

CONCLUSION: To our knowledge, this is the first reported case in Canada of the de novo development of daptomycin resistance with clini- 
cal failure to MRSA during therapy for endocarditis. Clinicians and microbiologists must be aware of this phenomenon given the implications for treatment and transmission of this strain. It also raises questions about the utility of daptomycin in settings of heavily pretreated patients with persistent MRSA bacteremia.

\section{F5}

\section{DISSEMINATION OF COMMUNITY-ASSOCIATED METHICILLIN-RESISTANT STAPHYLOCOCCUS AUREUS IN NORTHERN SASKATCHEWAN}

J Irvine ${ }^{1}$, B Quinn ${ }^{1}$, D Stockdale ${ }^{1}$, S Woods ${ }^{2}$, M Nsungu $^{2}$, P Levett ${ }^{3 *}$, R McDonald ${ }^{3}$, G Golding ${ }^{4}, \mathrm{G}$ Horsman $^{3}, \mathrm{M} \mathrm{Mulvey}^{4}$ and the Northern Antibiotic Resistance Partnership (NARP)

${ }^{1}$ Population Health Unit, LaRonge; ${ }^{2}$ Northern Inter-tribal Health Authority, Prince Albert; ${ }^{3}$ Saskatchewan Disease Control Laboratory, Regina, SK; ${ }^{4}$ National Microbiology Laboratory, Winnipeg, MB

OBJECTIVE: Although much attention has focused on community associated methicillin resistance in large urban centres in Canada, some reports have described the emergence of CA-MRSA in northern communities. This study examines the incidence of CA-MRSA in the northern half of Saskatchewan.

METHODS: Surveillance was conducted over six years beginning in 2001 in three of the most northerly Saskatchewan health regions for all communities (on and off-reserve). Specimens from clinical indications were collected from remote community health centers and small rural hospitals ( all $<35$ beds) and MRSA positive cases were reported to the respective health authorities. Cases with asymptomatic carriage were excluded. In order to calculate total CA-MRSA rates of recurrence, cases occurring more than 2 months of the preceding episode and / or at a different site, were considered a recurrent episode. Pulsed-field gel electrophoresis (PFGE) of Smal digested genomic DNA and RT-PCR for the mecA, nuc, and PVL-encoding genes was used to characterize a subset of the isolates. RESULTS: A total of 1,927 MRSA events in 1,409 individuals were reported over the study period. Of these about $99 \%$ were clinically community-associated. Fifty-six percent $(\mathrm{N}=783)$ of the individuals were $<20$ years of age, while the majority of their cases $(80.8 \%)$ were skin and soft tissue infections. Of the CA-MRSA cases, $15.1 \%$ of the individuals had at least one recurrent episode after 2 months. The annual rate of CA-MRSA distinct individuals reported in these health regions increased from 9 per 10,000 population in 2001 (range to $4-10$ per 10,000 ) to 169 per 10,000 in 2006 (range 43-233 per 10,000). An annual periodicity was observed with the highest number of cases being reported during the third quarter (JulySeptember). A subset of strains ( $\mathrm{N}=192)$ were typed and $97.4 \%(\mathrm{~N}=187)$ were found to be Canadian PFGE epidemic type CMRSA7 (USA400) with 3 strains being CMRSA2 (USA100/800) and 2 strains being CMRSA10 (USA300).

CONCLUSION: This report describes the rapid emergence of CA-MRSA in Northern Saskatchewan and can assist in the development of interventions. The molecular epidemiology appears to be different from urban centers in southern Canada with the majority of cases being caused by CMRSA7.

\section{F6}

\section{EVALUATION OF OUTPATIENT TREATMENT OF CA-MRSA IN SKIN AND SOFT TISSUE INFECTIONS IN A LARGE HEALTH AUTHORITY}

T Leung ${ }^{1}$, A Lakhani ${ }^{1}$, L Cheng ${ }^{3}$, Y Arikan² ${ }^{2}$ D Purych ${ }^{2 *}$, A Grunfeld 1 ${ }^{1}$ Surrey Memorial Hospital, Surrey, BC/Fraser Health Authority; ${ }^{2}$ Royal Columbian Hospital, New Westminster, BC/Fraser Health Authority; ${ }^{3}$ Burnaby Hospital, Burnaby, BC/Fraser Health Authority OBJECTIVE: The increasing prevalence of community-associated methicillin resistant Staphylococcus aureus (CA-MRSA) requires clinicians to evaluate optimal therapy to achieve successful clinical outcomes. The primary objective of the study was to evaluate the prevalence of CA-MRSA in skin and soft tissue infections (SSTI) treated with outpatient therapy in four emergency departments (ED). Secondary objectives were to evaluate antimicrobial use and make recommendations for empiric antibiotic therapy based on the culture and susceptibility $(C \& S)$ results from the patient population.

METHODS: A retrospective chart review of patients presenting to the EDs with a SSTI was conducted. Patients were included if they were over the age of 18 , diagnosed with a skin and soft tissue infection, and were evaluated in the ED, ED treatment room care or admitted to the Home IV Program. Patients were excluded if they were diagnosed with polymicrobial, diabetic foot or severe infections requiring hospital admission.

RESULTS: A total of 472 patients were included in the study. C\&S information was available for 194 patients. CA-MRSA was isolated from 87 out of 194 patients (45\%). Of these, 100\% were susceptible to trimethoprim/sulfamethoxazole and therefore presumed to be CA-MRSA. Of the presumed CA-MRSA isolates, $100 \%$ were susceptible to mupirocin, $98 \%$ to rifampin, $97 \%$ to fusidic acid and doxycycline, and $80 \%$ were susceptible to clindamycin. Only $57 \%$ of patients with cultures positive for CA-MRSA had therapy specifically targeted to CA-MRSA. Group A Streptococcus was found in 29 of 194 cultures (15\%), and $100 \%$ were susceptible to ampicillin, penicillin, cefazolin and cephalexin and $71 \%$ to clindamycin.

CONCLUSION: The prevalence of CA-MRSA was found in almost half of the cultures, making the organism important to target for empiric therapy. Susceptibility results suggest clindamycin is not an optimal choice as a first line empiric therapy for SSTIs in EDs. Physician education was then targeted toward incision and drainage of abscesses as part of the treatment protocol, local epidemiological and susceptibility data, and appropriate selection of antibiotics based on presentation.

\section{Oral Presentations \\ Friday, February 29}

\section{Session G}

\section{G1} DETERMINATION OF THE COMPLETE GENOMIC SEQUENCE OF THE HUMAN PARAINFLUENZA VIRUS 4 (HPIV4)

C Yea ${ }^{1}$, R Cheung ${ }^{2}$, C Collins ${ }^{2}$, D Adachi ${ }^{2}$, J Nishikawa ${ }^{1}$, R Tellier ${ }^{1,2 *}$ ${ }^{1}$ Research Institute Hospital for Sick Children; ${ }^{2}$ Division of Microbiology Hospital for Sick Children, Toronto, ON

OBJECTIVE: Human parainfluenza viruses are distributed between 2 genera in the subfamily Paramyxovirinae. To date, no complete genomic sequence for HPIV4 has been determined. Following the isolation in our laboratory of a HPIV4 from a clinical sample, we aimed to determine the complete genomic sequence.

METHODS: The initial isolate was grown in primary rhesus monkey kidney cells (RMK) and subsequently passaged in LLC-MK2 cells. The isolate was initially identified by electron microscopy, immunofluorescence, and sequencing of an amplicon from the nucleocapsid gene. The complete sequence was obtained by sequencing amplicons obtained by long RTPCR, 5'RACE and RNA ligase assay using RNA extracted from passage 5. RESULTS: From a nasopharyngeal swab sample we grew a virus initially detected by hemagglutination. The isolate was not reactive with Mabs specific for influenza A and B, parainfluenza 1,2 and 3, RSV or adenovirus. By electron microscopy particles with the typical morphology of paramyxoviruses were observed. The isolate was reactive with a commercial Mab specific for HPIV4 (Chemicon) and sequencing of a fragment of the nucleocapsid gene identified the isolate as HPIV4 subgroup B. Several overlapping large amplicons have been used to obtain a near full length sequence of 15,321 nt. The sequencing of the genome terminii is underway.

CONCLUSION: This is the first complete genomic sequence of HPIV4 to be determined, and it represents a valuable contribution to the description of the human virome. Detailed analysis of the sequence will be presented. 


\section{G2}

\section{MAMMALIAN REASSORTANTS CONTAINING THE NS GENE FROM THE B ALLELE CAN TRANSMIT BETWEEN MAMMALS; THE MYSTERY OF B ALLELE RESTRICTION CONTINUES}

T Hatchette ${ }^{1,2 *}$, R Webby ${ }^{3}$, P Willson ${ }^{4}$, R Webster ${ }^{3,5}$

${ }^{1}$ Department of Pathology and Laboratory Medicine, QEII HSC; ${ }^{2}$ Department of Pathology Dalhousie University, Halifax, NS; ${ }^{3}$ Division of Virology, Department of Infectious Diseases, St. Jude Children's Research Hospital, Memphis, TN, United States; ${ }^{4}$ Canadian Centre for Health and Safety in Agriculture, University of Saskatchewan, Saskatoon, SK; ${ }^{5}$ Department of Pathology, University of Tennessee Health Science Center, Memphis, TN, United States

OBJECTIVE: The influenza A NS gene is unique because it has evolved into two distinct alleles. Viruses with the A allele have been found in different avian and mammalian species whereas the B allele is restricted to avian species. Why the B allele has not established itself in mammalian species is unclear. We hypothesize that viruses containing the NS B allele are unable to efficiently transmit between mammalian hosts which has prevented them from establishing a stable lineage in mammals.

METHODS: Single gene reassortants were generated using the eightplasmid reverse genetics system; one containing the avian A allele NS gene from $\mathrm{A} /$ chicken/Calfornia/139/01 and the other containing the B allele NS gene allele from $\mathrm{A} /$ chicken/California/6643/01. The remaining genes were derived from A/swine/Texas/4199-2/98 a strain of H3N2 circulating among US pigs. The resulting reassortants (Tx98-139 and Tx986643) were compared in a swine transmission model where six 3-4 week old pigs were inoculated with $10^{6} \mathrm{EID}_{50}$ of virus intra-nasally. The pigs were placed back in a single pen with 6 un-inoculated littermates. Clinical signs of disease and body temperature for each pig were monitored twice daily, and nasal swabs were collected from all pigs each day for the 14 day experimental period. Lungs were harvested for pathologic comparison at day 14. Each virus was assessed in separate experiments.

RESULTS: None of the pigs developed clinically apparent disease after exposure to influenza virus and all groups grew at an equivalent rate. The pigs that were exposed by contact to Tx98-139 had higher body temperature $(\mathrm{p}<0.05)$ and more lung pathology than those exposed by contact to Tx98-6643 $(\mathrm{p}<0.002)$. However, there was no difference in the peak titres shed from pigs exposed to either reassortant. Although there was a 2 day lag in the time the contact animals exposed to littermates infected with the reassortant containing the $\mathrm{B}$ allele begin shedding virus compared to those exposed the reassortant containing the A allele, this was not significant $(\mathrm{p}>0.1)$.

CONCLUSION: Using a pig transmission model we were unable to demonstrate any difference in shedding titres or time to transmission in pigs exposed to littermates infected with single gene reassortants containing NS genes from either the A or B allele

\section{G3} PREVALENCE OF HIGH RISK HPV GENOTYPES IN
ONTARIO WOMEN DETERMINED BY AMPLICOR $₫$ HPV
PCR AND LINEAR ARRAY OF SUREPATH $₫$ PAP SAMPLES M Chernesky' ${ }^{1 *}$, D Jang ${ }^{1}$, E Portillo' ${ }^{1}$, M Smieja ${ }^{1}$, S Chong ${ }^{1}$, S Buracond ${ }^{1}$, J Kapala ${ }^{2}$, J Patel ${ }^{2}$, C Doucette ${ }^{2}$, J Sumner ${ }^{2}$ ${ }^{1}$ St. Joseph's Healthcare/McMaster University, Hamilton; ${ }^{2}$ GammaDynacare Medical Laboratories, Brampton, ON

OBJECTIVES: The AMPLICOR HPV PCR [AMP] and Linear Array HPV genotyping tests [LA] from Roche have recently been approved for the diagnosis of HPV in Canada. There is limited published data on their performance on liquid-based SurePath Pap samples [L-Pap]. We tested L-Pap samples from women seeking routine cervical cancer screening using AMP and LA to determine concordance for HR types, reproducibility of AMP results between two laboratories and distribution of genotypes in the population.

METHODS: Samples from patients attending Ontario medical clinics were Pap-tested and within 1 week the residual L-Pap fluid from patients with abnormal cytology were extracted in QlAamp MinElute columns. Amplification and detection of HR HPV and beta-globin DNA were performed according to the package inserts. For the reproducibility protocol the two laboratories extracted and tested a panel of samples. Analysis was performed using SPSS software.

RESULTS: A total of 325 women have been enrolled [49\%>30 yr]. Sixty-five percent [212] contained HR types. The following genotype prevalences were found: 16 [24\%]. 18 [8\%], 51 [11\%], 52 [10\%], 39 [9\%], 56 [8\%], 31 or 58 [6\%], 33 [4\%], 35, 45 or 68 [3\%], 59 [2\%]. Of the 212 samples containing an HR genotype, 44 had 2, 17 had 3, 7 had 4, and 1 had 5 different types. HPV DNA was detected in 100\% [15/15] samples with HSIL, 83.0\% with ASCUS [78/94] and 81.7\% with LSIL [107/131]. In patients $<30$ years of age, types 16 or 18 were found in $38.9 \%$ of ASCUS, $46.2 \%$ of LSIL and $80 \%$ of HSIL compared to $27.8 \%, 14.7 \%$ and $33.3 \%$ respectively in those $>30$. The tests agreed $79.7 \%$ of the time with the AMP test finding more positives due to a higher analytical sensitivity. The 2 laboratories agreed on positives and negatives $76.2 \%$ of the time.

CONCLUSIONS: This ongoing study showed that although infections with types $31,39,51,52,56$ or 58 were prevalent in $52 \%$ of the positive samples, they were often accompanied by types 16 and 18 , which were more often associated with cytological diagnoses of ASCUS or HSIL. Results of AMP testing between laboratories may be due to differences in extraction and/or performance of the assay.

\section{G4}

PEPTIDE MIMETICS - A NOVEL CLASS OF INHIBITOR THAT BLOCKS INFLUENZA A AND B VIRUS REPLICATION

\section{J Mahony*, L Liu}

McMaster University, St. Joseph's Healthcare, Hamilton, ON

OBJECTIVE: Current therapies for influenza virus infections include M2 channel inhibitors (Amantadine) and neuraminidase inhibitors (Oseltamivir). Emerging resistance to these inhibitors may necessitate the development of novel classes of inhibitors. The RNA-dependent RNA polymerase (RdRNP) of influenza virus consists of a heterotrimeric complex of three subunits (PA, PB1 and PB2). The PB1 subunit contains both $\mathrm{PA}$-and PB2-binding domains whose peptide sequences have been identified. The objective of the study was to determine whether peptide mimetics based on these binding domains can inhibit virus replication.

METHODS: We have targeted the $\mathrm{N}$-terminal 18 amino acid residues of the PB1 subunit which represents the PA-binding domain of PB1. We made a synthetic 25 amino acid peptide including the cognate 18 aa PAbinding domain followed by a 7 aa (PKKKRKV) polybasic nuclear localization signal (NLS) to allow the peptide to cross the cytoplasmic and nuclear membranes to the site of viral RNA replication. The peptide was tested for its ability to inhibit influenza transcription and replication by DFA staining of infected MDCK cell monolayers using an influenza monoclonal antibody (DHI) 24 post infection and by testing infected cell culture supernatants for infectious virus by plaque assay.

RESULTS: PB1 peptide inhibited RdRNP activity and synthesis of matrix protein by up to $90 \%$ when used at sub-millimolar concentrations. PB1 peptide inhibited virus replication by $>90 \%$ as assessed by plaque assay. Influenza $\mathrm{A}$ and $\mathrm{B}$ replication were inhibited by 92 and $68 \%$ respectively by 0.5 uM PB1 peptide and inhibition was dose dependent when peptide was added either $90 \mathrm{~min}$ prior to or $60 \mathrm{~min}$ after infection.

CONCLUSION: Influenza virus RdRNP activity and virus replication were both inhibited by a PB1 subunit peptide mimetic that disrupts RNA polymerase. Sequence analysis of over 40 influenza strains indicates that the PA-binding domain of the PB1 peptide is highly genetically conserved and predicts that all influenza A viruses including H5N1 should be inhibited by this peptide. If effective in animal model studies, this novel class of inhibitor may warrant phase I clinical trials in humans. 


\section{Session $\mathrm{H}$}

\section{H1}

\section{RISK FACTORS FOR ACQUSITION OF ENDEMIC BLASTOMYCOSIS}

M Choptiany ${ }^{1 *}$, L Wiebe ${ }^{2}$, B Limerick ${ }^{2}$, P Sarsfield ${ }^{2}$, M Cheang ${ }^{1}$ B Light ${ }^{1}$, G Hammond ${ }^{1}$, K Macdonald ${ }^{3}$, E Trepman ${ }^{1}$, P Pappas ${ }^{4}$, J Embil ${ }^{1}$

${ }^{1}$ University of Manitoba, Winnipeg, MB; ${ }^{2}$ Northwestern Health Unit; ${ }^{3}$ Lake of the Woods District Hospital, Kenora, ON; ${ }^{4}$ University of Abama, Birmingham, Alabama, United States

OBJECTIVE: Blastomycosis is a potentially fatal fungal infection, but environmental risk factors for acquiring blastomycosis have not been well established. The objective of this study was to better define risk factors for the acquisition of endemic blastomycosis in our geographic area.

METHODS: A cross-sectional questionnaire survey involving 112 patients with history of blastomycosis and 200 control subjects in Manitoba and northwestern Ontario.

RESULTS: The most common tissues involved with blastomycosis lesions were pulmonary, skin and soft tissues, and bone. A significantly greater proportion of patients with blastomycosis than control subjects were male, between 31 and 60 years old, and involved in outdoor occupations. A significantly greater percentage of patients with blastomycosis than control subjects were immunosuppressed either from collagen vascular disease or immunosuppressive therapy or had hypothyroidism. Patients with blastomycosis had a significantly longer smoking history than control subjects. Having coworkers infected with blastomycosis was significantly associated with development of blastomycosis. However, specific activities, specific occupations, occupational factors such as farming or digging, and having a pet dog with blastomycosis were not associated with an increased risk of infection. After excluding the non-significant predictors in a series of backward stepwise modeling, four factors were determined to be independent predictors of developing blastomycosis: immunosuppression for any reason, including drugs or disease (adjusted OR, 5.7; 95\% CI, 1.4 to 22.5; $\mathrm{P}=0.013$ ), being an outdoor worker (adjusted $\mathrm{OR}, 3.1 ; 95 \% \mathrm{CI}, 1.5$ to 6.2 ; $\mathrm{P}=0.0014$ ), smoking (pack-years) (adjusted OR, 1.0; 95\% CI, 1.0 to 1.0; $\mathrm{P}=0.027$ ), and having a coworker with blastomycosis (adjusted OR, 5.9; 95\% CI, 1.5 to $23.4 ; \mathrm{P}=0.012$ ).

CONCLUSIONS: Independent risk factors for the development of blastomycosis included immunosuppression for any reason (including drugs or disease), being an outdoor worker, smoking (pack-years), and having a coworker with blastomycosis.

\section{$\mathrm{H} 2$}

THE CLINICAL MANIFESTATIONS AND OUTCOMES OF PEDIATRIC BLASTOMYCOSIS IN CHILDREN AND YOUTH: A 30 YEAR EXPERIENCE

S Fanella, S Skinner*, J Embil

Department of Medical Microbiology, University of Manitoba, Winnipeg, MB

OBJECTIVE: Blastomyces dermatitidis is a thermally dimorphic fungus endemic to areas of North America. It can cause a granulomatous infection affecting every organ system. Limited data exists about the clinical details of pediatric blastomycosis infections. A summary of pediatric cases over the last 30 years at our institution was conducted.

METHODS: Retrospective chart review, spanning the past 30 years, of 34 pediatric patients with a laboratory diagnosis of blastomycosis, who were admitted to a pediatric centre for management of blastomycosis. Demographic, clinical and diagnostic data was evaluated.

RESULTS: The mean age of cases was $10.0 \pm 5.4$ years, 20 were male. The majority $(59 \%)$ resided in Northwest Ontario. Six cases had documented direct exposure to soil and 3 had dogs diagnosed with blastomycosis. The most common symptoms prior to admission included cough (76\%), fever (74\%), fatigue (54\%) and weight loss (27\%). The mean time from symptom onset to clinical diagnosis was 60.7 days (range 1-365 days). Length of hospitalization was $16.1 \pm 12.7$ days. Methods of diagnosis included bronchoscopy
(10), abscess culture (9), induced sputum (7), brain biopsy (3), lung resection (3) and gastric aspirate (2). Pulmonary involvement was observed in $28(82 \%)$ and $12(35 \%)$ had 1 or more extra-pulmonary manifestations, with $5(15 \%)$ central nervous system (CNS), 9 (26\%) skin/soft tissue and $6(18 \%)$ bone episodes. Chest $\mathrm{x}$-rays were abnormal in $28(82 \%)$, with the left lower lobe most commonly affected (32\%). Initial therapy with Amphotericin B was required in 19 cases. Readmission relating to blastomycosis was required $5(15 \%)$ cases. There were no deaths from blastomycosis and $1 / 3$ of cases had residual deficits.

CONCLUSIONS: In our sample blastomycosis had a similar presentation as previously reported. A notable proportion had CNS involvement. To our knowledge this represents the largest series of pediatric blastomycosis, and includes clinical and outcome data. Blastomycosis should be included in the differential of chronic pneumonia, and other non-resolving infections in children who live or visit areas endemic for $B$. dermatitidis.

\section{H3}

\section{CHYLOUS ASCITES: A LATE COMPLICATION OF INTRAABDOMINAL MYCOBACTERIUM AVIUM COMPLEX IMMUNE RECONSTITUTION SYNDROME IN HIV- INFECTED PATIENTS}

P Phillips ${ }^{1 *}$, J Lee ${ }^{2}$, C Wang ${ }^{3}$, E Yoshida ${ }^{4}$, J Montaner ${ }^{5}$

${ }^{1}$ Division of Infectious Diseases, British Columbia Center for Excellence in HIV/AIDS, St. Paul's Hospital; ${ }^{2}$ Department of Medicine, University of British Columbia, Vancouver, BC; ${ }^{3}$ Division of Gastroenterology, Univerisity of Toronto, Toronto, ON; ${ }^{4}$ Division of Gastroenterology, Vancouver General Hospital; ${ }^{5}$ Division of AIDS, British Columbia Center for Excellence in HIV/AIDS, St. Paul's Hospital, University of British Columbia, Vancouver, BC OBJECTIVE: To report a case series of chylous ascites related to M. avium complex intra-abdominal immune reconstitution syndrome (MAC-IRS) in HIV-infected patients. This complication is extremely rare, with only 6 previously reported cases.

METHODS: This is a retrospective study from a single institution.

RESULTS: During the past 6 years, chylous ascites was diagnosed in 6 (32\%) of 19 AIDS patients, all of whom had previously been diagnosed with intra-abdominal non-tuberculous mycobacterial infection (NTMIRS). A review of medical records (1994-2007) identified no other cases of chylous ascites among HIV-positive patients over 13 years. The incidence of chylous ascites was estimated at 1 in 2,300 HIV-positive admissions. All patients presented with abdominal distention; and 5 also had abdominal pain. The ascitic fluid had a milky appearance and a median triglyceride level of $4.07 \mathrm{mmol} / \mathrm{L}$ (range 3.19 to $29.6 \mathrm{mmol} / \mathrm{L}$ ) $(360 \mathrm{mg} / \mathrm{dl}$, range 282 to $2,620 \mathrm{mg} / \mathrm{dl}$ ). Median CD4 counts before initiation of highly active antiretroviral therapy (HAART), then at the time of initial diagnosis of MAC-IRS, and finally when chylous ascites developed were 10, 75, and 130 cells/ L, respectively. Compared to baseline, the median reduction in HIV RNA at the time when chylous ascites developed was $3.3 \log _{10}$ copies $/ \mathrm{mL}$. Patients had MAC previously recovered from blood ( $\mathrm{n}=6$ cases), abdominal lymph nodes $(n=5)$, and duodenal biopsies $(n=4)$. Ascitic fluid specimens for mycobacterial smears and cultures were positive for only 1 and 2 of the patients, respectively. The median duration of symptomatic ascites was 9 months (range 2 to 15 months). After a median follow-up of 20 months, 5 (83\%) of 6 patients survived.

CONCLUSIONS: Chylous ascites is a rare and late complication of intra-abdominal MAC-IRS, developing in $32 \%$ of our patients with intraabdominal NTM-IRS. This appears to be associated with a favourable prognosis, with the use of specific MAC and HIV therapy, even in the presence of clarithromycin-resistant MAC. Of note, the incidence of chylous ascites in our HIV-positive patients is over five-fold higher than previously reported in the general population. 


\section{H4}

PATIENTS WITH CDAD DUE TO NAP1-RELATED STRAINS OF CLOSTRIDIUM DIFFICILE HAD STOOL TOXIN B LEVELS THAT WERE HIGH COMPARED TO THE AVERAGE FOR NAP1-UNRELATED STRAINS

M Alfa ${ }^{1,3 *}$, R Murray ${ }^{1}$, M Mulvey ${ }^{1,2}$, D Boyd ${ }^{2}$

${ }^{1}$ University of Manitoba; ${ }^{2}$ National Microbiology Laboratory;

${ }^{3}$ Diagnostic Services of Manitoba, Winnipeg, MB

INTRODUCTION: Clostridium difficile associated diarrhea (CDAD) due to NAP1-related strains has been reported to correlate with more severe disease. It has been suggested that hyperproduction of toxin by these strains may correlate with increased virulence. PFGE is often used to categorize clinical strains as NAP1-related but there have been no reports giving the quantitative levels of Toxin B in stools of patients with NAP1-related C. difficile strains.

OBJECTIVE: The objective was to assess stool to determine if patients with NAP1-related strains of C. difficile had higher levels of Toxin B compared to those with CDAD due to NAP1-unrelated strains. Furthermore, testing was performed to assess if the NAP1-related strains were hyperproducers of Toxin B in vitro.

MATERIALS AND METHODS: Stool samples that were GD antigen and Toxin A antigen positive and had been collected within 3 to 24 hours of toxin testing were included in this evaluation. The titre of Toxin B in the stool samples was assessed using the CPE assay. C. difficile isolates were obtained from stool samples by alcohol shock and culture on CDMN agar. The titre of spores post-alcohol shock was also assessed for stools that were $<3$ hours old. PFGE analysis was performed for all isolates and profile comparison was done using Bionumerics software.

RESULTS: There were 29 unique patient stool samples analyzed for Toxin B titre within 24 hours of collection. PFGE analysis of the isolates obtained showed there were two NAP1-related strains, 13 NAP2 strains, one NAP4, NAP6 and NAP7 strains as well as 11 strains that had unique profiles that did not match a NAP designation. The Toxin B levels that ranged from $10^{1}$ to $10^{5} \mathrm{CPE}$ units/gm faeces (mean Toxin B titre was $4 \log _{10}$ CPE units/gm faeces). The stools that grew NAP1-related strains had Toxin $\mathrm{B}$ titres of $10^{4}$ and $10^{5} \mathrm{CPE}$ unit/gm faeces. The median spore level in stool samples tested within 3 hours of collection was $5.84 \log _{10}$ spores/gm faeces (range; 4.41-8.16 $\log _{10}$ spores/gm faeces).

CONCLUSIONS: The Toxin B titres for the NAP1-related strains were at the high end for in vivo (i.e. stool) levels in patients with CDAD. There was significant variation in Toxin $\mathrm{B}$ titres and spore concentration in stools from CDAD patients.

\section{Session I}

\section{1}

\section{IT'S ALL IN THE FAMILY: A CASE CLUSTER OF CA-MRSA FURUNCULOSIS, WITH A COMPLICATION OF POSSIBLE FAMILIAL TRIMETHOPRIM-SULFAMETHOXAZOLE (TMP SMX) HYPERSENSITIVITY}

L Saxinger*

University of Alberta, Edmonton, $\mathrm{AB}$

OBJECTIVES: Describe a case series of recurrent CA MRSA related furunculosis within a family, presenting with probable TMP SMX aseptic meningitis and hypersensitivity in three family members.

METHODS: Retrospective chart review.

RESULTS: A 4 member family sequentially developed recurrent CA MRSA furunculosis over a 8 month period, and all were given high dose oral TMPSMX. The father presented with symptoms suggestive of TMPSMX induced aseptic meningitis- headache, photophobia, fever, nausea and rash, and the two children had headaches, photophobia, nausea, and the son also had a rash. Subsequently the father, mother and daughter were confirmed to have CA MRSA infection. Decolonization (oral and topical) was offered and all follow up swabs were negative, with no recurrences 4 months later.

CONCLUSION: CA MRSA is presenting in our community in cases outside the classical "risk factor" groups, and disease without nose/groin carriage is seen. Issues regarding location of carriage of CA MRSA and screening protocols are discussed. TMPSMX related aseptic meningitis is discussed. Better data regarding which components of decolonization are most important are needed to guide management of recurrent CA MRSA furunculosis.

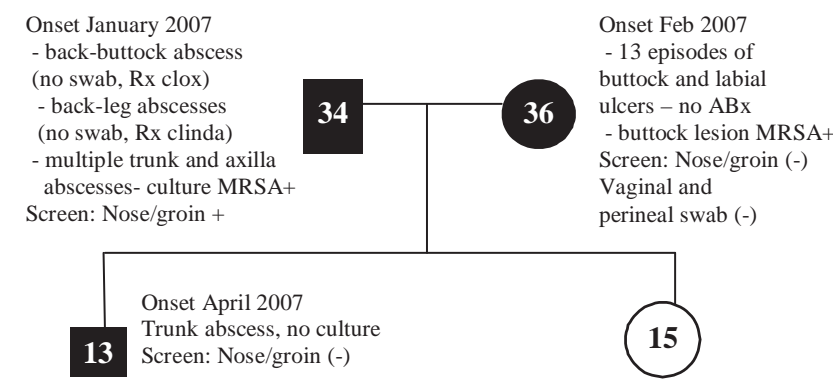

12

METHICILLIN-RESISTANT STAPHYLOCOCCUS AUREUS

IDENTIFIED IN HOSPITALIZED PATIENTS WITH NO RISK FACTORS FOR HEALTHCARE-ASSOCIATED TRANSMISSION M Mulvey $^{1 *}$, M Ofner ${ }^{2}$, J Campbell ${ }^{1}$, G Golding ${ }^{1}$, C Watt $^{3}$, L Louie $^{3}$, A Simor ${ }^{3}$, M Loeb ${ }^{4}$, A McGeer ${ }^{5}$, E Bryce ${ }^{6}$, A Matlow $^{7}$ and the Canadian Nosocomial Infection Surveillance Program (CNISP) ${ }^{1}$ National Microbiology Laboratory, Winnipeg, MB; ${ }^{2}$ Public Health Agency of Canada, Ottawa; ${ }^{3}$ Sunnybrook Health Sciences Centre, Toronto; ${ }^{4}$ McMaster University, Hamilton; ${ }^{5}$ Mount Sinai Hospital, Toronto, ON; ${ }^{6}$ Vancouver General Hospital, Vancouver, BC;

${ }^{7}$ Hospital for Sick Children, Toronto, ON

OBJECTIVES: Community associated methicillin resistant Staphylococcus aureus (CA-MRSA) is an emerging issue particularly in North America. The objective of this work was to determine the incidence of CA-MRSA cases in hospitalized patients and determine phenotypic and genotypic characteristics of the strains identified.

METHODS: Cases were identified through the Canadian Nosocomial Infection Surveillance Program (CNISP). The study took place over six months beginning in January 1, 2006. When a new case of MRSA was identified, the patient's medical records were reviewed for clinical and epidemiologic data. A CA-MRSA case was defined as an MRSA identified from an admitted patient with no established health-care associated risk factors, including, (i) MRSA identified $<48$ hours after admission; (ii) no history of hospitalization, surgery, or dialysis within 1 year of MRSA culture; (iii) no residence in a long term care facility within 1 year of MRSA culture; (iv) no indwelling catheter or medical device; (v) no prior MRSA. Strains were characterized by PCR for the mecA, nuc, and PVL genes, and typed using PFGE, spa, and SCCmec typing. Antimicrobial susceptibilities were determined to 10 antimicrobials using microbroth dilution according to CLSI.

RESULTS: 414 CA-MRSA cases were identified in 37 of 50 (74\%) hospital sites. The distribution of cases in Canada were as follows: Western region $(\mathrm{N}=255 ; 61.6 \%)$; Ontario and Quebec $(\mathrm{N}=147 ; 35.5 \%)$; Eastern region $(\mathrm{N}=12 ; 2.9 \%) .22$ cases of necrotising pneumonia were identified. PVL toxin was identified in $177(57.5 \%)$ strains. 58.4\% ( $\mathrm{N}=180)$ of the strains would be classified as community strains (CMRSA7 or CMRSA10), 32.5\% ( $\mathrm{N}=100)$ would be considered typical hospitalacquired (HA) strains, and 9.1\% ( $\mathrm{N}=28)$ did not cluster using PFGE. No vancomycin resistant strains were identified. The CA-MRSA strains were less likely to be resistant to clindamycin $(\mathrm{P}<.001)$ and ciprofloxacin $(\mathrm{P}<.014)$ as compared to the HA-MRSA strains identified in the community.

CONCLUSIONS: Although CA-MRSA strains containing PVL were identified in the majority of cases, typical HA-strains were also found circulating in the community. It will be important to continue to monitor this trend to see if CA-MRSA strains replace the HA-MRSA strains circulating in the community. 


\section{I3}

THE DIABETIC FOOT CLINIC: NOT A SIGNIFICANT SOURCE FOR METHICILLIN-RESISTANT STAPHYLOCOCCUS AUREUS (MRSA) ACQUISITION.

P Lagacé-Wiens ${ }^{1 *}$, D Ormiston ${ }^{1,2}$, J Embil ${ }^{1,2,3}$, L Nicolle ${ }^{1,3}$, T Hilderman ${ }^{4}$

${ }^{1}$ University of Manitoba, Department of Medical Microbiology and Infectious Diseases; ${ }^{2}$ Health Sciences Centre, Infection Prevention and Control; ${ }^{3}$ Health Sciences Centre, Department of Internal Medicine; ${ }^{4}$ Manitoba Health, Winnipeg, MB

BACKGROUND: MRSA represents a significant clinical and infection prevention and control issue in healthcare facilities and increasingly in the community. The impact of outpatient clinics on MRSA transmission remains unclear, although diabetic foot clinics have been implicated in the spread of MRSA. The objective of this study was to determine if attendance at a tertiary care centre based diabetic foot clinic resulted in transmission of MRSA to its attendees.

METHODS: We reviewed medical records and laboratory data for all attendees of the Health Sciences Centre Diabetic Foot and Complicated Wound Clinic (DFCWC), Winnipeg, Manitoba, from October 1997 through October 2007. At the DFCWC, 1500 - 2500 patient visits occur annually. All clinic specimens yielding MRSA and new to the facility were considered. Charts and laboratory data from new MRSA patients were reviewed and patients without evidence of previous MRSA infections or colonization documented from an external laboratory or hospital site and having at least one prior clinic visit were presumed to have acquired their MRSA at their initial or follow-up visits. Clinic and clinic-attributable incidence were compared to provincial and hospital incidence for the same time interval.

RESULTS: Ninety-one MRSA cases new to the hospital were identified during the 10 year period. Of these, 85 were either known to be colonized or infected with MRSA prior to or identified at the first visit to the DFCWC. Six new isolations were probable clinic acquired cases. PFGE of these isolates revealed a non-clonal distribution (3 CMRSA-2, one CMRSA-1 and two unique profiles). The ten year trend of total in-clinic MRSA incidence paralleled the trend of total hospital and provincial incidence, demonstrating an increase over time. Clinic-attributable incidence was low and the trend was stable.

CONCLUSIONS: Contrary to other reports, it does not appear as if the DFCWC plays a role in the transmission of MRSA to its attendees. While the data presented in this study is reassuring, suggesting that transmission of MRSA within this population is low, confirmation of the concept can only be achieved in a future prospective study. The infection prevention and control practices in place, appear to be appropriate for limiting inclinic acquisition of MRSA.

\section{4}

\section{HIGH PREVALENCE AND PERSISTENCE OF METHICILLIN RESISTANT STAPHYLOCOCCUS AUREUS (MRSA) IN A DOWNTOWN VANCOUVER COMMUNITY}

M Hull1,2*, E Lloyd-Smith ${ }^{1}$, S Champagne ${ }^{2}$, M Tyndall1', SH Goh ${ }^{3}$, R Zhang ${ }^{1}$, P Daly ${ }^{4}$, M Romney ${ }^{2}$

${ }^{1}$ British Columbia Centre for Excellence in HIV/AIDS; ${ }^{2}$ Providence Health Care; ${ }^{3}$ British Columbia Centre for Disease Control; ${ }^{4}$ Communicable Disease Division, Vancouver Coastal Health, Vancouver, BC

OBJECTIVES: Community-Associated MRSA (CA-MRSA) has been identified as an emerging pathogen in North America, and inner-city residents in Vancouver have been found to have higher rates than the general population. We undertook a study to investigate persistence of CA-MRSA carriage using repeated surveillance screens of a defined community-based sample.

METHODS: Residents of 3 single-occupancy inner-city hotels were recruited for MRSA prevalence screening between January and April 2006, and subsequently in June and July 2007. Surveillance samples were obtained from nares and any open wounds and cultured for MRSA using selective, chromogenic media. Molecular analyses were performed on all isolates identified as MRSA and the presence of CA-MRSA markers, namely SCCmecIV, PVL and PFGE type, was evaluated.

RESULTS: 165 residents were screened (76\% uptake). The population was predominantly male $(71 \%)$ and the median age was 45 . A total of 55 (33\%) were nares or wound culture positive for MRSA and 43/55 (26\% of population) were confirmed to be CA-MRSA carriers (SCCmecIV and PVL positive). For CA-MRSA isolates, 13/165 (7.8\%) were isolated concurrently from wound and nasal swabs, $24(15 \%)$ were from nares specimens only, and $6(4 \%)$ were from wounds without nasal colonization. $61 \%$ had prior visits to the Emergency Department in the last 12 months, and $35 \%$ reported admission to hospital. A total of $96(58 \%)$ residents participated in both prevalence screens. Amongst these residents, 33 (34\%) demonstrated persistent MRSA colonization, 21 (22\%) acquired MRSA and $13(14 \%)$ were no longer found to carry MRSA at time of second screen.

CONCLUSION: Community-based screens of an inner-city population reveal ongoing evidence of high level MRSA colonization. A majority of the MRSA is of the CA-MRSA type. Community carriage of MRSA appears to be a persistent phenomenon in downtown Vancouver, and only a minority of participants demonstrated clearance of MRSA. Further studies evaluating the effectiveness of community-based decolonization strategies in high MRSA prevalence populations are needed.

\section{Sesson J}

\section{J1}

\section{UPDATE ON DIAGNOSIS OF STRONGYLOIDIASIS: A CLINICIAN'S PERSPECTIVE}

M Ndao ${ }^{1 *}$, F Vasquez-Camargo' ${ }^{1}$, R Varatharajalu², D MacLean ${ }^{1}$, F Neva ${ }^{2}$, B Ward ${ }^{1}$

${ }^{1}$ McGill University/National Reference Centre for Parasitology/Tropical Diseases Centre, Montreal, QC; ${ }^{2}$ Laboratory of Parasitic Diseases, National Institute of Allergy and Infectious Diseases, National Institutes of Health, Bethesda, MD, United States

OBJECTIVES: The diagnosis of strongyloides remains difficult and the consequences of a missed diagnosis can be serious. The clinical features of infection can be subtle and non-specific. Both standard ova and parasite examinations and more specialized clinical laboratory concentration (Agar plate, Baermann extraction, Harada Mori culture, charcoal culture) are a biohazard and are insensitive compared to strongyloides serology. The sensitivity of the ELISA using crude antigen is high, however the specificity is low due to antibody cross-reactivity to antigens present in other parasites, such as filarial parasites, Ascaris lumbricoides, hookworm and Schistosoma spp.

METHODS: We compared three S. stercoralis antigens (one recombinant and 2 crude) with both ELISA and immunoblot. To improve sensitivity, specificity, and provide better automated diagnosis of strongyloidiasis, surface-enhanced laser desorption ionization time-of-flight mass spectrometry (SELDI-TOF-MS) serum protein profiling technology was used to identify specific candidate biomarkers. Sera from four clinical subsets were tested (S. stercoralis larva-positive patients, HTLV1 S. stercoralis positive patients, eosinophilic esophagitis cases and travelers/immigrants judged at risk for Strongyloides but negative for Strongyloides larvae).

RESULTS: With the ELISA all three antigens demonstrated 100\% sensitivity in the strongyloides larva-positive, (with or without HTLV1 positivity). The specificity for the crude batch 1 , crude batch 2 and recombinant antigens was 67,75 and $85 \%$ respectively. The immunoblot using the recombinant antigen showed a specificity of $98 \%$. The SELDI-TOF preliminary experiments revealed significant differences in protein peak intensity between all disease groups when analysed by Ciphergen Biomarker Wizard ${ }^{\mathrm{TM}}$ software.

CONCLUSIONS: Further measures of specificity with various helminthes are in progress. This recombinant antigen has the benefit of reproducibility. The ELISA with the recombinant antigen is sensitive and the Western blot appears specific. 


\section{J2}

\section{A LONGITUDINAL STUDY ON SUB-POPULATIONS OF CRYPTOSPORIDIUM SPP. IN SPORADIC HUMAN CASES IN BRITISH COLUMBIA, CANADA \\ J Lee, $C$ Ong* \\ University of British Columbia, Vancouver, BC}

OBJECTIVE: Cryptosporidiosis is a parasitic gastro-intestinal disease that has occurred as large waterborne outbreaks in several communities in British Columbia (BC). In between outbreak periods, sporadic cases of cryptosporidiosis have also been reported. As the disease is not endemic in $\mathrm{BC}$, this study aims to determine whether there is any association between sporadic and outbreak-related cases using a molecular epidemiological marker, gp60.

METHODS: Fecal specimens were collected from sporadic cases with laboratory-confirmed cryptosporidiosis in the Lower Mainland region of BC. Amplicons of approximately $1100 \mathrm{bp}$ spanning a hypervariable region of the gp60 locus were obtained using PCR and sequenced bi-directionally. Isolates were assigned allelic subtypes based on the comparison of assembled sequences to reference sequences in public databases using multiple sequence alignment and phylogenetic cluster analysis.

RESULTS: Seven sub-populations corresponding to the 2 major gp60 allelic groups of C. hominis (Ib and Id) and 3 alleles (IIa, IIc and IId) of C. parvum were identified in 16 sporadic isolates collected over a 4 year period (1996-1999). Of these, the Ib allele with the IbA10G2 subtype was the most prevalent and was found in $25 \%$ (4 of 16) of the isolates. This gp60 subtype was also the same one found in isolates from cases associated with 2 large waterborne outbreaks (Kelowna \& Penticton) in BC in 1996. By 1997, only 1 isolate had the Ib allele but this was a different subtype (IbA12G3). In 1999, 3 years after the outbreaks in the Okanagan, this IbA12G3 subtype was found in another C. hominis isolate. In contrast, the most prevalent C. parvum gp60 subtype was IIaA15G2R1 found in $25 \%$ (4 of 16) cases, 3 in 1997 and 1 in 1999. This C. parvum subtype was one of the prevalent subtypes reported in infected cattle in Ontario.

CONCLUSION: The different sub-populations of Cryptosporidium spp. that were identified in sporadic cryptosporidiosis cases indicated that transmission of this disease in a 3 year period after the occurrence of several large waterborne outbreaks was largely via secondary spread during the $1^{\text {st }}$ year. However, transmission by other routes including zoonotic spread was more prevalent in subsequent years.

\section{J3}

\section{EPIDEMIOIOGY OF STAPHYLOCOCCUS AUREUS} BLOODSTREAM INFECTIONS IN A LARGE CANADIAN HEALTH REGION, 2000-2006

K Laupland ${ }^{1 *}$, T Ross ${ }^{2}$, D Gregson ${ }^{1,2}$

${ }^{1}$ Department of Medicine; ${ }^{2}$ Calgary Laboratory Services, Calgary,

AB

OBJECTIVES: A number of reports have suggested that the epidemiology of invasive Staphylococcus aureus infections may be changing. Our objective was to describe the epidemiology of $S$. aureus bacteremia and assess whether the incidence, severity, and antimicrobial resistance rates are increasing.

METHODS: Population-based surveillance for all incident S. aureus bacteremias was conducted in a large Canadian health region (population 1.2 million) during 2000-2006.

RESULTS: The annual incidence was $18.5 / 100,000$ and it slightly decreased during the first five years of the study and then increased. Forty one percent of cases were nosocomial, $34 \%$ healthcare-associated community onset, and $25 \%$ were community acquired. While rates of healthcare associated community onset and nosocomial methicillin-sensitive $S$. aureus (MSSA) bacteremia were similar throughout the study, rates of community-acquired MSSA bacteremia gradually decreased. In contrast, rates of methicillin-resistant $S$. aureus (MRSA) bacteremia dramatically increased during the study. These were predominantly CMRSA-2 but CMRSA-10 (USA300) strains were increasingly isolated especially from community onset infections since 2004. Most patients (77\%) had significant chronic co-morbid illnesses and/or alcoholism. Dialysis dependence, organ trans- plantation, HIV infection, cancer, and diabetes were the most important risk factors, and were comparable for MSSA and MRSA bacteremias. Bacteremia without focus, bone and joint, soft tissue, and lower respiratory tract infections were the most common clinical diagnoses. The overall case fatality was $25 \%$ and was higher for those with MRSA (39\%) as compared to MSSA ( $24 \% ; \mathrm{p}<0.0001)$. However, the annual overall population mortality rate for $S$. aureus bacteremia did not significantly change.

CONCLUSIONS: Although the overall incidence, risk factors, and outcomes of S. aureus bacteremia has not significantly changed in the past 7years, MRSA has emerged as an important cause of bacteremia in our region.

\section{Sesson K}

\section{K1}

VALIDATION OF REAL TIME PCR ASSAYS FOR DETECTING AND SEROGROUPING N. MENINGITIDES BEFORE IMPLEMENTATION IN A ROUTINE MOLECULAR DIAGNOSTIC LABORATORY

T Chiu $^{1 *}$, R McDonald ${ }^{2}$, N Antonishyn ${ }^{2}$, G Tyrrell ${ }^{1}$, L Chui ${ }^{1}$

${ }^{1}$ ProvLab, Edmonton, AB; ${ }^{2}$ Saskatchewan Disease Control Laboratory, Regina, SK

INTRODUCTION: Neisseria meningitides (N.M) is one of the most important invasive bacterial pathogens worldwide. Invasive meningococcal disease (IMD) is characterized by a rapid progression from mild symptoms to septicaemia and/or meningitis, Due to the prompt advancement of IMD, a rapid diagnostic test such as PCR is critical. The objective of this study was to independently validate an in-house real time PCR assay for identifying and serogrouping N.M.

METHODS: Analytical sensitivity for all PCR targets was determined by assaying serial dilutions of a standardized cell suspension of N.M spiked with CSF, whole or lysed blood. DNA was extracted using the GC12 Magtration system (PSSBio). The ctrA gene target was used to identify N.M. Specificity of the PCR assay was determined by using DNA isolated from a panel of different bacterial reference strains. Primers and probes for detection of serogroups A, B, C, W135, or Y were validated against 26 confirmed N.M isolates of varying serogroups collected from different body sites.

RESULTS: The primers and probes for the identification and serogrouping of N.M were highly specific with no cross activity detected in any of the bacterial species included in the panel. The primers and probes targeting the $c$ trA gene had a detection limit of 10 cells/reaction in CSF and 100 cells/reaction in whole blood and lysed blood. For serogrouping, the detection level was at 1 cell/reaction in CSF and 10 cells/reaction in whole blood and lysed blood. The primers and probes for each serogroup were specific, and no cross reactivity was observed. The same PCR reaction was adapted onto the LightCycler (Roche) using Taqman master kit (Roche) and identical results were obtained. The TAT was under $4 \mathrm{~h}$ when the assay was performed using the ABI Sequence Detection System, and a reduction of 90 min was seen when the assay was performed on the LightCycler (Roche).

CONCLUSIONS: In our laboratory, the performance of this in-house real time PCR assay for N. meningitidis is highly specific and sensitive for identification and serogrouping. The TAT of the assay is less than $4 \mathrm{~h}$ and could be easily implemented into a routine molecular diagnostic laboratory. 


\section{K2}

DEXAMETHASONE (DEX) IN CRYPTOCOCCUS GATTII CENTRAL NERVOUS SYSTEM INFECTION (CGCNS) P Phillips ${ }^{1 *}$, K Chapman ${ }^{2}$, M Sharp ${ }^{2}$, P Harrison ${ }^{3}$, J Vortel ${ }^{4}$, T Steiner ${ }^{5}$, W Bowie $^{5}$, E Galanis ${ }^{6}$

${ }^{1}$ Division of Infectious Diseases, St. Paul's Hospital; ${ }^{2}$ Division of Neurology, St. Paul's Hospital; ${ }^{3}$ Department of Radiology, St. Paul's Hospital; ${ }^{4}$ Infectious Diseases, Richmond General Hospital; ${ }^{5}$ Division of Infectious Diseases, Vancouver General Hospital; ${ }^{6}$ British Columbia Centre for Disease Control, Vancouver, BC OBJECTIVE: To report the experience with Dex in the management of CgCNS infection.

METHODS: A retrospective review was completed for 5 patients (from 3 hospitals) treated with Dex during the course of CgCNS infection.

RESULTS: From August 2004 to 2007, we identified 5 patients with confirmed CgCNS who were treated with Dex. The median age was 47 yr (range 40-63), and none had known T-cell immune defects. The baseline cerebrospinal fluid (CSF) findings (available for 4 of 5 cases) included a median opening pressure (OP) of $37 \mathrm{~cm}$ water (range 14-60), WBC $307 \mathrm{M} / \mathrm{L}$ (range 60-607), and all had cryptococcal antigen (CRAG) titres $\geq 1: 1024$, India ink smear (Ink) and culture positive for $\mathrm{Cg}$. Brain imaging findings included lesions compatible with cryptococcomas ( $n=3$ patients) and enhancing lesions in the basal ganglia associated with dilated perivascular spaces and edema $(n=2)$. Cases $1-4$ were given a trial of Dex (median initial dose $12 \mathrm{mg} /$ day, and median duration 63 days) after a median duration of 51 days of antifungal therapy (range 26-56) because of worsening or persisting mental status abnormalities $(\mathrm{MS})(\mathrm{n}=4)$ and lesions on brain scan $(n=3)$, at which time OP was $\leq 26$ $\mathrm{cm}$ water, and all cases had sterile CSF for a median of 39 days (range 1154), but still had a median CSF CRAG of $\geq 1: 1024$ and were Ink positive $(n=2$ of 4$)$. Case 5 , initially considered to have neoplastic disease received Dex for the 2 weeks prior to initiation of antifungal therapy. A favourable clinical response was observed (Cases 1-4) at a median of 2 days (range 1-14), and radiologic improvement (Cases 1, 3, and 4) at 9 . 13 days after starting Dex. The response for Case 3 was confounded by simultaneous placement of a ventriculoperitoneal shunt; however brain mass lesions were also improved after 13 days of Dex. Case 5 experienced deterioration in MS and brain lesions on imaging 2 mo. after stopping Dex but while continuing AmB +5-FC (total 63 days), suggesting the possibility of an immune reconstitution syndrome.

CONCLUSIONS: Although corticosteroids are not recommended for management of cryptococcal meningitis, these observations suggest that Dex should be further evaluated in CgCNS infection, particularly in patients whose clinical response to antifungal treatment is unsatisfactory despite CSF sterilization and control of intracranial pressure.

\section{K3}

\section{ARE INDIRECT IMMUNOFLUORESCENCE ANTIBODY ASSAYS (IFA) RELIABLE TOOLS FOR THE DIAGNOSIS OF CAT SCRATCH DISEASE?}

B Serhir*, C Sauvé, S Beaulieu, M Pelletier, M Couillard Laboratoire de santé publique du Québec, Institut national de santé publique du Québec, Sainte-Anne-de-Bellevue, QC OBJECTIVES: Bartonella henselae is associated with Cat Scratch Disease (CSD), a common cause of regional lymphadenopathy. Although molecular methods are highly sensitive and specific for the diagnosis of CSD, they require tissue and are of limited availability. Hence, the diagnosis of CSD is most frequently based on serology. The aim of this study was to evaluate the performance of two Health Canada approved B. henselae-IFA kits.

METHODS: Two kits, the Focus Diagnostics Bartonella IFA IgG and the Euroimmun Anti-Bartonella henselae IIFT (IgG) were compared to an inhouse IFA assay that uses B. henselae antigenic slides from Bion Enterprises. A panel of 79 negative and 139 positive sera was selected based on the results obtained by the Bion reference method.

RESULTS: The results are summarized in the table.

\begin{tabular}{lcccccc} 
Bion & \multicolumn{3}{c}{ Euroimmun } & \multicolumn{3}{c}{ Focus } \\
& Pos. & Neg. & Ind. $^{*}$ & Pos. & Neg. & Ind. $^{*}$ \\
\hline Positive & 128 & 9 & 2 & 114 & 0 & 2 \\
Negative & 16 & 62 & 1 & 25 & 20 & 5 \\
\hline
\end{tabular}

*Specimens giving indeterminate results

The sensitivity and specificity of the Focus kit were $98 \%$ and $40 \%$ while the corresponding values were $92 \%$ and $78 \%$ for the Euroimmun kit. Seven (7) specimens gave inconclusive results with Focus compared to 3 specimens with Euroimmun because of non-specific- or auto-fluorescence bindings. The concordances of the Focus and the Euroimmun IFA results with the in house assay were $80 \%$ and $87 \%$, respectively.

CONCLUSION: Both commercial kits were sensitive but not specific for the detection of B. henselae IgG. The Euroimun IFA kit demonstrated a better performance than the Focus Kit. Considering the limitations of the serologic assays for the diagnosis of CSD, obtaining pertinent clinical information is of major importance for the evaluation and interpretation of laboratory tests.

K4

\section{THE METEORIC RISE OF MRSA AS A PROPORTION OF STAPHYLOCOCCUS ISOLATES FROM SKIN AND SOFT TISSUE INFECTIONS IN TWO OUTPATIENT IV CLINICS, EDMONTON, CANADA}

\section{Saxinger ${ }^{1 *}$, C Pisani ${ }^{2}$, J Fuller ${ }^{1}$, U Chandran ${ }^{2,1}$}

${ }^{1}$ University of Alberta; ${ }^{2}$ Dynacare Kasper Medical Laboratories, Edmonton, AB

OBJECTIVE: To describe the increase in Methicillin Resistant Staphylococcus aureus (MRSA) as a proportion of Staphylococcus aureus isolates from skin and soft tissue infections (SSTIs) treated in two tertiary hospital based Outpatient IV Therapy Clinics (OPAT clinics), against the background of the proportional increase in the community and non tertiary community hospital isolates.

METHODS: Laboratory data systems based review of 1) MRSA as a proportion of all Staphylococcus aureus isolates from community based clinics and hospitals, and one tertiary OPAT site, 2004-6 and January to November, 2007 and 2) All Staphylococcus aureus isolates from a new OPAT clinic at a second tertiary hospital, July through November 2007. Antibiogram data was used to inform the likelihood of the preponderance of community acquired (CA-MRSA) vs hospital acquired (HA-MRSA) isolates.

RESULTS: The background proportion of MRSA in community isolates rose from 2.9 to 7.8 percent over 2004 - 2006, with Clindamycin susceptibility of MRSA isolates rising from $50 \%$ to $73 \%$ suggesting increased CAMRSA. The established OPAT clinic site had an increase in MRSA proportion of SA from $7 \%$ to $26.7 \%$ over the same time period, with 36 MRSA isolates from 2004-6. From January to November 2007, there were an additional 26 MRSA isolates, which comprised $37.7 \%$ of the SA isolates. In the new OPAT clinic at another tertiary hospital established July 2007, until November 2007 there were 12 MRSA of 29 SA isolates from SSTIs, or $41.4 \%$. Antibiotic susceptibility data suggest the overwhelming majority are CA-MRSA.

CONCLUSIONS: These data indicate that MRSA from clinical specimens have been rising in Edmonton since 2004 with an abrupt increase in 2007 of CA-MRSA, with findings consistent between two high volume SSTI treating OPAT clinic sites. Standard empiric therapy from EDs is still betalactam based and cultures are inconsistently sent. There is a urgent need to use local epidemiology to guide antimicrobial utilization by establishing treatment frameworks for primary care and Emergency physicians who initiate treatment empirically, with education about the enhanced pathogenicity, clinical presentations, and beta lactam resistance of these isolates. 


\section{Session L}

\section{L1}

MAINTAINING TEMPERATURE OF BLOOD CULTURE SPECIMENS DURING TRANSPORT TO THE LABORATORY A NOVEL APPROACH FOR COLD CLIMATES

K Dirse*, M Carlson, G Johnston, P Brown

Stratford General Hospital, Stratford, ON

OBJECTIVE: Blood cultures should be delivered to the laboratory within two hours of collection and should never be refrigerated or frozen once inoculated. As the trend to centralization of Microbiology services continues, this two-hour window is often exceeded. In winter months, blood culture specimens may be subjected to inappropriate cold temperatures during transit. Our Microbiology service in the InterHospital Laboratory Partnership (IHLP), in South West Ontario, uses a BacT/Alert ${ }^{\circledR}$ blood culture system by bioMérieux. The manufacturer's recommendation is to maintain inoculated blood culture bottles at room temperature (RT) if delay in processing occurs. The objective of this study was to devise and validate a method of maintaining inoculated blood culture bottles at RT during transport.

METHOD: In fall 2006, we devised appropriate packing in our courier specimen bags and utilized commercially available air activated heat packs (Hothands - $2 \AA$ 10-hour hand warmers by HeatMax, USA) to maintain inoculated blood culture bottles at RT throughout transport. A continuous monitor probe device (temperature recorder by Fisher Scientific) was inserted into the specimen area of the zippered nylon courier bag. The bag was subjected to a variety of conditions and temperatures were recorded for a 5 hour period, the maximum transport time within the IHLP. During the 2006/07 winter season, random, periodic checks of the bags were made to assure RT conditions were maintained.

RESULTS: The heat packs were found to maintain the temperature inside the bag to within $5^{\circ} \mathrm{C}$ of the starting temperature with a range of $17-23^{\circ} \mathrm{C}$. Without a heating device the temperature inside the bag dropped significantly to as low as $9^{\circ} \mathrm{C}$.

CONCLUSIONS: With readily available, economical, commercial air activated heat packs in an appropriately configured specimen courier bag, the IHLP consolidated Microbiology Laboratory devised a system that maintains inoculated blood culture bottles at RT during transit in winter. In addition, this system was determined to be appropriate for transporting other RT specimens such as fluids and anaerobic cultures. We have been able to meet good laboratory practice standards.

\section{L2}

\section{EVALUATION OF ANTIMICROBIAL ACTIVITY OF THE ESSENTIAL OIL OF THE GUM OF PISTACIA VERA AND ITS MAJOR COMPONENTS}

\section{J Behravan*, BF Kalalinia, M Ramazani, A Asadipour}

Biotechnology and Pharmaceutical Research Centers, School of Pharmacy, Mashhad University of Medical Sciences, Mashhad, Islamic Republic of Iran

OBJECTIVE: The antibacterial and antifungal properties of essential oils have been studied for many years. There are reports of the active principles of essential oils from various plants with antibacterial or antifungal activity. However, less attention was given to the activities of their main components in the oils tested. The objective of this study was to evaluate the antimicrobial properties of essential oil of the gum of Pistacia vera and its major components.

METHODS: The essential oil from the gum of Pistacia vera grown in Iran was obtained by a hydro-distillation method. The antimicrobial activities of the oil and its major components $\alpha$-pinene, $\beta$-pinene, $\alpha$-thujone and $\beta$-thujone against the growth of five bacteria (Staphylococcus aureus, Bacillus subtilis, Escherichia coli, Klebsiella pneumonia and Pseudomonas aeruginosa) was evaluated by a microdilution method. Antifungal activities were measured against pathogenic yeast Candida albicans using an agar dilution method. Gentamicin and ketoconazol were used as controls for antibacterial and antifungal activities respectively.
RESULTS: Minimum Inhibitory Concentrations (MIC) of the oil against Staphylococcus aureus, Bacillus subtilis, Escherichia coli and Candida albicans were $2.34,15.57,93.40$ and $11.68 \mathrm{mg}_{\mathrm{ml}}{ }^{-1}$ respectively. The oil did not show any inhibitory effects against Klebsiella pneumonia and Pseudomonas aeruginosa. The major components of the oil, $\alpha$-pinene, $\beta$-pinene, $\alpha$-thujone and $\beta$-thujone were most active against Staphylococcus aureus (MICs of 1, 1,4 and $1 \mathrm{mg} / \mathrm{ml}$ respectively). The components were less active against other organisms. Pseudomonas aeruginosa was the most resistant organism to the inhibitory effects of the essential oil major components.

CONCLUSION: The oil and components were most active against Gram positive bacteria. However, they had lower activity than those of standard antibiotics, gentamicin, and ketoconazol under the conditions studied.

\section{L3}

\section{BI-ANNUAL SURVEILLANCE OF STAPHYLOCOCCUS AUREUS SUSCEPTIBILITY TO FUSIDIC ACID IN FIVE CANADIAN LABORATORIES}

R Rennie ${ }^{1}$, C Brosnikoff ${ }^{1 *}$, M Harris ${ }^{1}$, L Turnbull ${ }^{1}$, P Kidson ${ }^{2}$, D Yamamura ${ }^{3}$, C Bechard ${ }^{3}$, M Kelly ${ }^{4}$, B Waldron ${ }^{4}$, B Ta ${ }^{4}$, K Forward $^{5}$, K Lambert ${ }^{5}$, K Hewitt ${ }^{5}$, MC Locas $^{6}$, M Laverdiere $^{6}$ ${ }^{1}$ University of Alberta Hospital, Edmonton, AB; ${ }^{2}$ LEO Pharma, Thornhill; ${ }^{3} \mathrm{MDS}$ Laboratory Services, Toronto, ON; ${ }^{4} \mathrm{MDS}$ Metro Laboratory Services, Burnaby, BC; ${ }^{5} \mathrm{QE}$ II Health Sciences Center, Halifax, NS; ${ }^{6}$ Hopital Maisonneuve-Rosemont, Montreal, QC OBJECTIVE: The objective of this study was to perform on-going semiannual surveillance of fusidic acid susceptibility of Staphylococcus aureus strains in five Canadian diagnostic laboratories.

METHODS: S. aureus strains were collected from routine cultures submitted to the routine laboratory during March and September of the past year. Sixty (60) strains were collected from each of the five diagnostic laboratory sites; 10 strains from each site were methicillin resistant Staph aureus (MRSA) isolates. Only one strain per patient was tested in each test period. Routine antimicrobial susceptibility testing was performed in the submitting laboratory by an automated susceptibility method. Testing for fusidic acid susceptibility was performed by disk diffusion (Oxoid, Nepean, Ontario) and ETest ${ }^{\circledR}$ (AB Biodisk, Solna, Sweden) on Mueller Hinton agar plates. The plates were incubated at $35^{\circ} \mathrm{C}$ for $18-20 \mathrm{hr}$ in ambient air. A zone diameter of $>20 \mathrm{~mm}$ and an $\mathrm{MIC}$ of $<2 \mathrm{mg} / \mathrm{L}$ were used to indicate susceptibility of the $S$. aureus strains to fusidic acid.

RESULTS: 603 S. aureus strains were tested for fusidic acid susceptibility during 2007, including 100 MRSA strains. 187/603 (31\%) of strains were collected from inpatients and 416/603 (69\%) from outpatients. 22/300 (7.3\%) were resistant to fusidic acid in March and 32/303 (10.6\%) in September. $3 / 50(6 \%)$ strains of MRSA were resistant to fusidic acid in March and 2/50 (4\%) in September. All 5 strains of MRSA resistant to FA in 2007 were isolated from one laboratory.

CONCLUSIONS: Fusidic acid is the most widely prescribed topical antimicrobial in Canada. Compared to data gathered over the previous 7 years, ongoing surveillance suggests that there has been no trend towards increasing fusidic acid resistance in S. aureus strains isolated across the country. The resistance rate remains low even among the MRSA strains submitted for testing. These data support the importance of continuing surveillance and suggest that fusidic acid has maintained its efficacy over the years. 


\section{Oral Presentations Saturday, March 1}

\section{Session M}

\section{M1}

\section{SURVEILLANCE FOR LABORATORY-CONFIRMED INFLUENZA IN ADULTS HOSPITALIZED IN CANADIAN ACUTE-CARE HOSPITALS}

D Gravel $^{1 *}$, A McGeer ${ }^{2}$, G Taylor ${ }^{3}$, J Vayalumkal ${ }^{1}$, C Frenette $^{4}$ A Wong ${ }^{5}$, D Moore ${ }^{6}$, B Amihod ${ }^{7}$, S Eden ${ }^{1}$, C Weir ${ }^{1}$, Canadian Nosocomial Infection Surveillance Program Public Health Agency of Canada ${ }^{1}$

1Public Health Agency of Canada, Ottawa; ${ }^{2}$ Mount Sinai Hospital, Toronto, ON; ${ }^{3}$ University of Alberta Hospital, Edmonton, AB; ${ }^{4}$ Hopital Charles LeMoyne, Longueil, QC; ${ }^{5}$ Royal University Hospital, Saskatoon, SK; ${ }^{6}$ Montreal Children's Hospital, Montreal, QC; ${ }^{7}$ Jewish General Hospital, Montreal, QC

OBJECTIVES: To describe the epidemiology of influenza-associated illness in adults admitted to Canadian acute care facilities.

METHODS: Laboratory-based surveillance for influenza-associated illness was performed in 15 hospitals of the Canadian Nosocomial Infection Surveillance Program (CNISP) between November 1, 2006 and April 30, 2007. Eligible cases were in-patients $\geq 16$ years of age with a laboratory test (EIA, DFA, PCR or culture) positive for influenza. Cases were defined as hospital acquired (HA) if symptom onset was $>96$ hour after hospital admission, or If they were re-admitted $<96$ hours post-discharge.

RESULTS: A total of 173 cases of influenza were identified (165, 95\% influenza A; 8, 5\% influenza B). Median age was 66 yrs (range 16-98 yrs), 90 (52\%) were male. 129 (75\%) cases were community acquired (CA). Admitting diagnoses among CA cases were: pneumonia (54, 41\%), exacerbation of chronic lung disease/respiratory distress (24, 18\%), influenza/viral illness $(20,15 \%)$, sepsis/fever of unknown source $(10$, $8 \%)$ MI/CHF (10, $8 \%)$. The median rate of CA influenza was 1.07 per 1,000 admissions (range 0.20 to 2.30 per 1,000 admissions). The $44 \mathrm{HA}$ cases occurred in 7 hospitals, $22(50 \%)$ were part of the 3 identified influenza outbreaks. The median rate of HA influenza was 3.87 (range 0.00 to 15.53 ) per 100,000 patient days. Of the 173 cases of influenza, 15 (9\%) required ICU admission, 11 (6\%) required intubation/mechanical ventilation, and $14(8 \%)$ died within 30 days of symptom onset. $81 \%$ of HA cases, but only $36 \%$ of CA cases were treated with an antiviral; $44 \%$ of HA and $71 \%$ of CA cases also received an antibiotic. Most (122, 71 $\%)$ diagnosed cases were managed in droplet or droplet contact precautions in addition to routine practices, which for $79(46 \%)$ included a private room. N95 respirators or equivalents were used for $28(16 \%)$ patients.

CONCLUSIONS: Both CA and HA influenza are relatively common during influenza season. The majority of patients (85\%) with CAinfluenza had an alternate admitting diagnosis, highlighting the need for increased awareness of CA influenza in adults admitted to acute care hospitals. Influenza has been shown, in this report, to have a significant rate of serious complications and death.

\section{M2}

SASKATCHEWAN DISEASE CONTROL LABORATORY PANDEMIC PREPAREDNESS PLAN: MOLECULAR TESTING STRATEGY FOR INFLUENZA AND OTHER RESPIRATORY AGENTS

TA Maksymiw*, NA Crozier, ACA Obarianyk, TL Hansen, CM Bacalso, RR McDonald, NA Antonishyn, PN Levett, GB Horsman

Saskatchewan Disease Control Laboratory, Regina, SK

OBJECTIVES: Effective public health response during an influenza pandemic requires rapid identification of the pandemic strain and other respiratory pathogens. To meet this objective, we validated CDC influenza primers and other published primers using different extraction chemistries on three automated platforms (Qiagen, Kingfisher, Abbott m2000), and one manual method (Chelex). This strategy eliminates the waste normally associated with conventional stockpiling of reagents.

METHODS: We utilized real-time PCR for all targets. The matrix gene was chosen as a target for Influenza A screening to ensure the detection of all human and avian strains. Influenza A positives would be subtyped using $\mathrm{H} 1$ and $\mathrm{H} 3$ specific probes. Non-H1-H3 Influenza A positives, during the pre-pandemic phase, would be tested for $\mathrm{H} 5$ and H7. Simultaneous primary screening for other common respiratory agents was established or alternatively set up as a reflex test on Influenza A negatives. The following real-time PCRs have been established: Influenza B, hMPV, Parainfluenza 1-4, RSV A/B, Adenovirus, Chlamydia pneumoniae and Mycoplasma pneumoniae. Our strategy to meet the objective involved four components: 1) automate the extraction to increase throughput and surge capacity, 2) expand extraction options by validating methods with alternate reagent suppliers and robotic platforms, 3) automate master mix set-up, 4) establish reagent and quality control material stocks that will be used before expiry, while maximizing potential test volume.

RESULTS: We have established the capacity for 10,000 molecular tests without waste for 16 gene targets. Four automated extraction chemistries on three different platforms and an alternative crude manual extraction method have been validated for influenza specimen preparation. The implementation of robotics in conjunction with five real-time 96-well PCR instruments should permit the daily processing, with existing staff, of 184 respiratory specimens for eight potential agents of respiratory disease and typing as needed.

CONCLUSIONS: With border closure and supply shortages during an influenza pandemic, redundancy and automation with real-time PCR provides a reasonable approach to pandemic preparedness while eliminating potential waste in non-pandemic readiness.

\section{M3}

\section{DEFINING A REFERENCE STANDARD AND OPTIMIZING SAMPLING STRATEGY FOR INFLUENZA MOLECULAR DIAGNOSTICS}

M Smieja ${ }^{1,2 *}$, A Petrich ${ }^{1,2}$, K Luinstra ${ }^{1}$, S Buracond ${ }^{1}$, SA Chong1, J Mahony ${ }^{1,2}$

${ }^{1}$ St. Joseph's Hospital; ${ }^{2}$ McMaster University, Hamilton, ON

OBJECTIVE: Culture, the historic reference standard for assessing a new test, is less sensitive than molecular tests. Furthermore, efficient sampling strategies are needed to identify potentially positive specimens, to more precisely define diagnostic test characteristics of a new test at acceptable cost. Our objectives in this study were two-fold: 1 . To assess influenza test characteristics using a reference standard using 2 or more tests positive, and 2. To identify an efficient sampling technique to find additional positives.

METHODS: We tested 517 nasopharyngeal swabs submitted to the Regional Virology Laboratory at St. Joseph's Healthcare, Hamilton between November 2005 and April 2007. We chose samples by age- and month-stratified random samples. All samples were tested by direct fluorescent antibody (DFA) and by culture (if negative for influenza A, B and for six other respiratory viruses); by RVP multiplex PCR for 19 respiratory viruses (Luminex); and by the artus Influenza LC RT-PCR. Five groups of approximately 100 samples were created, including sampling by temporal matching, temporal-spatial matching, and sampling when influenza was not known to be circulating.

RESULTS: Of 517 NPS samples, DFA detected 84 positives for influenza; culture detected an additional 13; RVP detected 111; and artus detected 105. Using a traditional reference of DFA/culture positive, DFA was $86.6 \%$ sensitive and $100 \%$ specific; RVP was $95.8 \%$ and $95.7 \%$ specific; and artus was $94.8 \%$ sensitive and $96.8 \%$ specific. Using a reference standard of two tests positive (total 105 positive), the sensitivities were as follows: DFA: $79.0 \%$; DFA/culture $89.5 \%$; RVP 99.0\%; and artus $97.8 \%$. Specificity for RVP was $98.3 \%$, and $99.5 \%$ for artus. Examining various sampling strategies, the initial stratified random 
sample found 14 additional positives with RVP. Using temporal or temporal and spatial matching yielded an additional 2/185 positives (1\%). All 109 samples taken more than 6 weeks before/after influenza season were negative by all four tests.

CONCLUSIONS: A reference standard consisting of two tests positive found that DFA/culture is insensitive, whereas current commerciallyavailable molecular tests have high sensitivity and specificity. A stratified random sample was efficient in identifying additional positives, whereas more complex sampling strategies found very few extra positives.

\section{M4}

SELF-COLLECTED NASAL FLOCKED SWABS: THE NEXT GENERATION. A COMPARISON OF SELF-COLLECTED NASAL SWABS WITH STAFF-COLLECTED NASOPHARYNGEAL OR NASAL SWABS FOR RESPIRATORY TRACT SAMPLING IN VOLUNTEERS

S Castriciano*, S Carruthers, S Buracond, G So, M Smieja Department of Pathology and Molecular Medicine, McMaster University, Hamilton, ON

BACKGROUND: Nasal swab (NS) for diagnosis of respiratory viruses is less invasive than nasopharyngeal swabs (NPS), and may enable self collection. We studied 2 nasal swab prototypes, and found them equivalent to NPS in sampling epithelial cells, but self-collection was not as good as staff collection. In this study we modified one of the nasal flocked swab (NFS) to optimize sampling and comfort, and hypothesized that 2 sequential NS would be required for optimal respiratory cell sampling.

OBJECTIVE: To examine if the new Copan NFS is equivalent to NPS in sampling the upper respiratory tract, and if self-sampling is equivalent to staff sampling.

METHODS: 55 volunteers had 2 self-administered NFS, followed by 2 staff-administered NS using NFS or rayon swabs in randomized order. Pictorial instructions were provided. Discomfort, ease of administration, and preferences were assessed. The $2^{\text {nd }}$ self-collected swab was compared with the staff-collected swabs. 20 of the subjects had also 2 NPS collected using a pernasal FS or rayon swab. Swabs were placed in a one $\mathrm{mL}$ tube of UTM; 500 microliters were used to prepare cell smears. Respiratory epithelial cells were counted under an UV microscope; Averaging 4 fields or 10 fields when less than 10 cells per high-powered field (hpf) were present. RESULTS: In the 55 volunteers, the $2^{\text {nd }}$ self-collected NFS was superior to the initial swab, with a mean (SD) of 117 (65) vs. 67 (43) cells/hpf, $\mathrm{P}<0.001$. The self-collected NFS was superior to a staff-collected rayon NS, which sampled 38 (25) cells/hpf $(\mathrm{P}<0.001)$, and comparable to staffcollected NFS at 132 (56) cells/hpf $(\mathrm{P}=0.06)$. In the 20 subjects with NPS, the mean (SD) cell yields for NPS was 145 (43) and 55 (30) for the flocked and rayon, respectively $(\mathrm{P}<0.001)$; and 136 (53) for flocked and 32 (22) for rayon staff-administered NS $(\mathrm{P}<0.001$ for the rayon comparison; $\mathrm{P}=0.39$ for flocked NPS vs. flocked NS). Mild discomfort (58\%) no difficulty $(82 \%)$ in self-swabbing was reported. $40 \%$ preferred self-swabbing, $36 \%$ were neutral and $24 \%$ preferred staff collection.

CONCLUSIONS: The new Copan NFS design is superior to rayon NPS or NS, and equivalent to flocked NPS, for sampling respiratory tract epithelial cells. Self nasal sampling is feasible and easy to perform, and equivalent to staff sampling, 2 sequential swabs are required for optimal cell yield. Self-sampling could accelerate diagnosis of respiratory infections; a symptomatic patients study is needed.

\section{Session N}

N1

THE HTLV WESTERN BLOT REMAINS A POOR SUPPLEMENTARY TEST COMPARED TO PCR AND LEADS TO A HIGH NUMBER OF SAMPLES WITH INDETERMINATE BUT LIKELY NEGATIVE STATUS

L Malloch*, B Calder-Kent, Y Adonsu-Hoyi, K Kadivar, J Kim National HIV/AIDS Reference Lab, Ottawa, ON

OBJECTIVE: The NLHRS previously eliminated the HTLV western blot (WB) test from its algorithm approximately 5 years ago due to the higher than acceptable number of indeterminate final results from samples that were likely negative. Several Canadian labs performing HTLV testing have continued to use the WB test in their algorithms. We decided to re-examine this test and compared it against an HTLV PCR. Here we present results from this study.

METHODS: One-hundred (100) whole blood samples previously submitted and confirmed by HTLV PCR testing were used in this study. Sixtyeight (68) were diagnosed as HTLV positive and thirty-two (32) were diagnosed as HTLV-negative by HTLV PCR. Samples were processed for both proviral DNA and plasma which was stored at $-20^{\circ} \mathrm{C}$. The MP Diagnostics (formerly Genelab Diagnostics) HTLV Blot 2.4 was used according to manufacturer's instructions. The test uses a combination of cell-culture derived viral particles and recombinant proteins. These include MTA-1 and K55 to further distinguish HTLV-I and HTLV-II and GD21, a common yet specific protein for both virus types. The HTLV PCR testing algorithm includes two different in-house nested PCR tests that can distinguish between HTLV-I and HTLV-II.

RESULTS: Of the 68 samples previously diagnosed as HTLV-positive by PCR, 62 were also confirmed as positive by Western blot. WB test results from the other 6 were; 1 -negative, 1 -indeterminate, 4-HTLV pos but untypeable). Of the 32 samples diagnosed as HTLV-negative by PCR, 8 were diagnosed as negative by the western blot. However, 24 were diagnosed as indeterminate. Different banding patterns were observed but the most common cross-reactive bands included those from the gag ( $1919, \mathrm{p} 24, \mathrm{p} 28$, p53) and pol (p26, p32, p36) gene regions.

CONCLUSION: The HTLV western blot displayed very poor performance especially on samples previously diagnosed as negative by HTLV PCR. This would potentially have led to a significant number of reports with indeterminate test results and unresolved HTLV status.

\section{N2} HEPATITIS D VIRUS INFECTION IN CANADA: CURRENT PREVALENCE AND HDV GENOTYPES

K Swidinsky ${ }^{1 *}$, M Morbey ${ }^{1}$, E Grudeski ${ }^{1}$, K Kaita ${ }^{3}$, A Andonov ${ }^{1,2}$ ${ }^{1}$ Public Health Agency of Canada, NML; ${ }^{2}$ Department of Medical Microbiology, University of Manitoba; ${ }^{3}$ Health Sciences Centre, University of Manitoba, Winnipeg, MB

OBJECTIVE: Hepatitis delta virus (HDV) infection is not considered a major health problem among HBV infected patients in Canada although it is difficult to obtain a good estimation of its prevalence. There is no published data on HDV infection in Canada. The aim of this study is to determine its current prevalence and range of HDV genotypes in Canadian $\mathrm{HBs} A g$ positive patients with liver disease.

METHODS: Initial screening for HDV infection was performed by commercial enzyme immunoassay for anti-delta antibodies (International Immundiagnostics, US). Ongoing replication of HDV was determined by PCR, HDV-Ag and anti-HDV IgM testing.

RESULTS: Since 2003 laboratory diagnostics for HDV was centralized at one site in Canada which allowed the accumulation of sufficient number of HDV positive samples for analysis (over 2000 samples were submitted for HDV diagnostics). The average anti-HDV seroprevalence during the last five years was $7.9 \%$ (range $6.1 \%-9.9 \%$ ). No significant decline or increase in the prevalence of infection was identified during the period of observation. Majority of HDV infected patients (129 out of 210 or $61.4 \%$ ) were PCR positive. Presence of HDV RNA by PCR was a better marker of 
ongoing infection than anti-HDV IgM. Laboratory data indicates that the majority of HDV infections were chronic probably due to immigration of people from high endemic areas, although superinfection with HDV in some cases cannot be ruled out. Interestingly co-infection (based on coexistence of IgM anti-HBc with markers of HDV infection) was found in approximately one-tenth of all patients indicating a recent simultaneous infection with both viruses. HDV antigen could be considered as a very poor marker of infection (only $2.3 \%$ positivity among all HDV patients). Majority of the patients (88.7\%) were infected with HDV genotype I, 7\% with genotype II, $2.8 \%$ with genotype $\mathrm{V}$ and $1.4 \%$ with genotype IV.

CONCLUSION: This is the first comprehensive study of HDV infection in Canada and it shows that the disease is not vanishing. The observed prevalence may be biased due to the referral effect of patients with unknown liver disease. Search for HDV status at least once in the medical survey of an $\mathrm{HBs} \mathrm{Ag}$ positive patient should be encouraged.

\section{N3}

DNA SEQUENCE ANALYSIS OF HIV-1 NON-B SUBTYPES HIGHLIGHTS THE INCREASING GENETIC DIVERSITY OF HIV ENTERING CANADA AND POSES A CHALLENGE FOR THE DIAGNOSIS AND QUANTITATION OF THESE STRAINS J Kim*, Z Chen, J Mihowich, J Deschatelets, S Lavoie, K Pulido National HIV/AIDS Reference Lab, Ottawa, ON

OBJECTIVE: The presence of HIV-1 non-B subtypes is increasing worldwide. Our objective in this study was to perform DNA sequence analysis on HIV samples to determine the genetic subtype and then conduct a more detailed sequence analysis to examine the effect of genetic diversity on the ability of PCR and viral load kits to diagnose and quantitate these strains. This analysis clearly demonstrates that the genetic diversity in HIV strains entering Canada poses a diagnostic and clinical monitoring challenge and also highlights the potential for recombination of these strains into new and possibly more virulent strains.

METHODS: Samples submitted for HIV confirmation and consultation by molecular analysis are subjected to the NLHRS PCR testing algorithm. Samples confirmed as positive are then entered into a DNA sequencing algorithm. Depending on the genetic subtype obtained, samples are subjected to further DNA sequence analysis of specific genes (gag/pol). The effect of genetic diversity on HIV viral load testing is performed using the bioMerieux Nuclisens HIV-1 QT, Bayer bDNA v 3.0, Roche Cobas Amplicor Monitor v 1.5 and the new Abbott Real-Time HIV-1 assays.

RESULTS: Phylogenetic and recombinant identification analysis revealed samples with a wide range of genetic diversity. In addition to identifying so called non-B 'pure' subtypes, a troublesome finding is the presence of HIV-1 recombinants. The wide range of genetic diversity increases the potential for recombination and creation of strains with a replicative and possibly more virulent advantage. Sequence analysis of the gag gene region also revealed that extensive sequence divergence exists between these strains and the primers and probes used in two commercial assays that target the gag gene region, most notably the bioMerieux Nuclisens QT and Roche Cobas Amplicor HIV-1 assays leading to underquantitation of these strains which can have a major impact on clinical management.

CONCLUSION: There is now a high degree of genetic diversity in HIV already present in Canada. One potential danger is the recombination of these HIV into more virulent strains. We recommend that DNA sequencing of samples especially in the region targeted by primers and probes used in molecular diagnostic and viral load tests be incorporated into clinical management practices.

\section{N4}

VALIDATION OF AN HIV ANTIBODY AVIDITY ASSAY FOR DETECTION OF RECENT SERO-CONVERSION FOR USE IN HIV INCIDENCE ESTIMATIONS

D Taylor ${ }^{*}$, D Cook ${ }^{1}$, R Chow ${ }^{4}$, P Cook ${ }^{4}$, P Tsang ${ }^{4}$, M Gilbert ${ }^{1,2}$,

L Baker $^{3}$, M Krajden ${ }^{1,2}$

${ }^{1}$ British Columbia Centre for Disease Control; ${ }^{2}$ University of British Columbia, Vancouver, BC; ${ }^{3}$ Siemens Medical Solutions Diagnostics, Tarrytown, NY, United States; ${ }^{4}$ PHSA Laboratories, Vancouver, BC OBJECTIVES: To validate an HIV antibody avidity assay for detection of recent sero-conversion (s-c) for use in HIV incidence estimations.

METHODS: The standard HIV antibody test protocol for the Bayer ADVIA Centaur ${ }^{\mathrm{TM}}$ instrument (Siemens Medical Solutions Diagnostics) was modified to allow on-board re-testing of HIV antibody-positive samples diluted 1:20 in 2M guanidine and in PBS. Relative light unit (RLU) values were obtained and an avidity index (AI) was calculated by dividing the RLU in the presence of guanidine by the RLU obtained with PBS. Preliminary data indicated that an AI of $\leq 0.8$ correlates with HIV infection within the past $6 \mathrm{~m}$. Serum samples of known HIV status were tested: 1) 46 samples from acute HIV infections (p24 Ag positive and Western Blot negative or indeterminate); 2) 60 samples from 38 commercial s-c panels; $55 / 60$ of these samples were collected 76 days or less after infection; 3) 50 samples from patients known to have been infected more than 12 months previously; 4) 100 commercial samples from long-term HIV infections.

RESULTS:

\begin{tabular}{lccc} 
Sample type & $\mathbf{n}$ & $\mathbf{A l} \leq \mathbf{0 . 8}$ & Al > 0.8 \\
\hline Acute HIV infection & 46 & $34(74 \%)$ & $12(26 \%)$ \\
S/C Panels (commercial) & 60 & $60(100 \%)$ & $0(0 \%)$ \\
Long term infection & 50 & $1(2 \%)$ & $49(98 \%)$ \\
Long term infection (commercial) & 100 & $6(6 \%)$ & $94(94 \%)$ \\
\hline
\end{tabular}

Of the 46 acute HIV samples, 11 had very low or absent EIA test signals. When these samples are excluded, 34/35 (97\%) gave an Al of $\leq 0.8$.

CONCLUSIONS: The avidity assay described here has performance characteristics similar to other assays described in the literature that have been shown to accurately identify individuals whose HIV infection occurred within the previous $6 \mathrm{~m}$. The use of on-board dilution facilitates rapid turnaround of avidity tests. Avidity test data will be useful to derive incidence estimates for HIV infections from cross-sectional population testing.

\section{Session $O$}

\section{1}

BORDETELLA HOLMESII: HOW COMMON IS IT IN BRITISH COLUMBIA?

SH Goh ${ }^{1,3 *}$, R Chen ${ }^{2}$, D Chan ${ }^{2}$, B Auk ${ }^{2}$, C Chand ${ }^{2}$, A Paccagnella ${ }^{2}$, $\mathrm{A} \mathrm{MCNabb}^{2}$, L Hoang ${ }^{1,3}$

${ }^{1}$ University of British Columbia; ${ }^{2} \mathrm{PHSA}$ Laboratories; ${ }^{3}$ University of Britisih Columbia, CDC, Vancouver, BC

OBJECTIVE: Our current Bordetella pertussis PCR diagnostic test at the PHSA Laboratory, BCCDC is a block-based PCR targeting the IS481 repeat sequences of $B$. pertussis. This is the most commonly used molecular-based target for B. pertussis identification (ID) worldwide. However IS481 sequences have been found in B. holmesii and some strains of B. parapertussis, B. bronchiseptica, B. avium and B. petri. We review our in-house observations in British Columbia (BC) of IS 481 positive samples.

METHODS: The IS481 PCR was performed as reported by Glare et. al. (J. Clin. Microbiol. 28:1982-87, 1990). All other PCR tests used to ID B. pertussis and B. holmesii were either block-based or qPCR tests.

RESULTS: Over the last 24 months, we have identified seven IS481 positive $B$. holmesii clinical specimens/isolates during routine testing of specimens sent to our laboratory, of which 4 were respiratory clinical specimens. These samples were either B. pertussis culture negative or 
B. holmesii culture positive. We wish to report on three of these clinical cases where B. holmesii was isolated from (1) a blood culture, (2) a nasopharyngeal swab and (3) a blood culture and a nasopharyngeal (NP) wash in which the latter was positive for IS 481, but subsequently determined to be positive for both B. pertussis and B. holmesii. The last case is a first report of a mixed Bordetella pertussis/B. holmesii infection from a respiratory source that subsequently tested culture positive for B. holmesii alone through a blood culture from the same patient (3).

CONCLUSIONS: The $\mathrm{BC}$ data concurs with other published reports indicating that at least in some parts of Canada and the USA, B. holmesii can be isolated from immunocompetent patients with bacteremia or pertussive/whooping cough. Also there is a possibility that mixed B. pertussis/ B. holmesii nasopharyngeal specimens may be missed if IS 481 PCR is used alone as was the case for patient \#3. The sole use of an IS 481 target PCR may compromise the microbial diagnostic accuracy of such patients in regions with a higher prevalence of $B$. holmesii infections. Further surveillance using a second molecular target, concurrently with the IS 481 PCR may shed light on the potential regional clinical burden of such an organism.

\section{$\mathrm{O} 2$}

\section{A RECENT CASE OF INTESTINAL (INFANT) BOTULISM IN BRITISH COLUMBIA (BC): "WHERE ARE THE BOTULINUM SPORES COMING FROM?"}

J Fung ${ }^{1 *}$, J Isaac-Renton 1,2, J Wong1, B Wong1, E Brodkin ${ }^{3}$, E Galanis ${ }^{1}$ ${ }^{1}$ British Columbia Centre for Disease Control Laboratory Services; ${ }^{2}$ University of British Columbia, Vancouver; ${ }^{3}$ Fraser Health Authority, Surrey; ${ }^{4}$ British Columbia Centre for Disease Control Epidemiology Services, Vancouver, BC

OBJECTIVE: Intestinal or infant botulism is a rare disease that can affect otherwise healthy babies under 1 year of age. It is caused by ingestion of Clostridium botulinum spores with subsequent growth of the bacterium and in-vivo toxin production in the colon. Intestinal botulism is rare in BC, with 12 laboratory confirmed cases since 1985 . The most recent case was in October 2007. This report details laboratory investigation of intestinal botulism and in this case, the ensuing environmental study. Data from the 12 confirmed cases are also reviewed.

METHODS: Diagnosis of intestinal botulism is established by identification of Clostridium botulinum organism and/or detection of toxin in patient's feces. C. botulinum organism is identified through enrichment culture of feces followed by confirmation of toxin in culture supernatant by mouse neutralization tests. BCCDC Laboratory Services has always been the only laboratory in $\mathrm{BC}$ carrying out botulism diagnosis.

RESULTS: A clinical diagnosis on a 6 month old child was made after presenting with symmetrical descending paralysis. Antitoxin (BabyBIG®) was given based on the clinical presentation. A stool sample was examined and Clostridium botulinum Type A organism identified. A serum sample was also examined but no toxin was detected. To answer the question "Where are the botulinum spores coming from?", a detailed food and environmental history was taken. Subsequently, 6 surface swab samples and 2 infant food samples were cultured. No C. botulinum was detected.

CONCLUSIONS: The Type A intestinal botulism case was confirmed by identifying Clostridium botulinum organism in feces of patient, but examination of environmental and food samples failed to identify the spores. This is not unusual in cases of intestinal (infant) botulism and understanding the epidemiology of this infection remains unclear. A review of the 12 cases will be discussed.

\section{O3}

COMPARISON OF SELF-COLLECTED VAGINAL SWABS TO FIRST-VOID URINE FOR THE DIAGNOSIS OF C. TRACHOMATIS, N. GONORRHOEAE AND T. VAGINALIS USING APTIMA $®$ ASSAYS

M Chernesky ${ }^{1 *}$, D Jang ${ }^{1}$, E Portillo ${ }^{1}$, M Smieja ${ }^{1}$, C Macritchie ${ }^{2}$, C Pritchard ${ }^{3}$, D Maceachern ${ }^{3}$, R Ewert $^{4}$

${ }^{1}$ McMaster University/St. Joseph's Healthcare; ${ }^{2}$ Community Health Centre, Hamilton; ${ }^{3}$ Youth Centre, Ajax; ${ }^{4}$ Evergreen Health Centre, Toronto, ON

OBJECTIVES: Women may be infected with C. trachomatis [CT], N. gonorrhoeae [GC] or T. vaginalis [TV] without symptoms in the lower genital tract. Screening programs using non-invasive samples are required to identify and treat infections, to prevent upper genital tract complications. First void urine [FVU], the first $20 \mathrm{ml}$ of a micturition may be difficult to collect accurately. Our studies examined the ability of APTIMA [Gen-Probe] assays to detect CT, GC and TV nucleic acids in self-collected vaginal swabs compared to FVU. We also determined ease of collection and sample preferences.

METHODS: Women [ $\mathrm{n}=350$ ] self-collected 2 or more vaginal swabs [dacron wrapped or nylon flocked] and an FVU. The samples were tested by APTIMA Combo 2 for CT and GC and Analyte Specific Reagents [ASR] for TV (Gen-Probe). Questionnaires were administered to determine ease of collection.

RESULTS: Self-collected wrapped dacron and nylon flocked vaginal swabs performed equally well for the diagnosis of CT [\% sensitivities 88-100] and GC [\% sensitivity 100] compared to first-void urine [sensitivity $92.6 \%$ for CT and $80 \%$ for GC]. Self-collected vaginal swabs tested by ASR detected $83.3 \%$ of the women infected with TV. The majority of women reported that self-collecting swabs was very easy, and preferred swabbing over collecting urine.

CONCLUSIONS: Both the dacron kit swabs [Gen-Probe] and flocked swabs [Copan] were effective for women to easily collect their own vaginal samples, providing a sample type diagnostically more accurate than urine for screening for CT, GC and TV.

\section{O4}

QUANTITATIVE URINE CULTURE COLONY COUNTS AND INTERPRETATION UNCHANGED BY AUTOMATED STREAKING ON CHROMOGENIC MEDIA

P Lagacé-Wiens ${ }^{1 *}$, M Alfa ${ }^{1,2}$, O Larios ${ }^{1}$, K Manickam ${ }^{1,2}$, J Karlowsky ${ }^{1,2}$ ${ }^{1}$ University of Manitoba, Department of Medical Microbiology and Infectious Diseases; ${ }^{2}$ Diagnostic Services of Manitoba, Winnipeg, MB

OBJECTIVES: Quantitative urine cultures represent a significant proportion of the workload in clinical microbiology laboratories. Automation of plate streaking would reduce urine culture workload, but the preservation of quantitative accuracy using automated streaking devices for urine cultures remains unproven. The purpose of this study was to determine if automated plate inoculation for quantitative urine cultures has any impact on colony count and/or interpretation of results.

METHODS: The study had two parts. Initially, we compared colony counts arising from 1:100, 1:1000 and 1:10,000 dilutions of a 0.5 McFarland standard of ATCC strains of E. coli, E. faecalis and P. aeruginosa inoculated using a calibrated $0.001 \mathrm{~mL}$ loop and streaked manually on blood agar and MacConkey/blood agar split plates with those arising from a $0.001 \mathrm{~mL}$ inoculum streaked using an automated ISOplater ${ }^{\mathrm{TM}}$ on CHROMagar ${ }^{\mathrm{TM}}$ plates. In the second part, we compared colony counts arising from 1056 consecutive quantitative urine cultures inoculated onto MacConkey/blood agar split plates using manual streaking to automated streaking of CHROMagar ${ }^{\mathrm{TM}}$ plates using the ISOplater ${ }^{\mathrm{TM}}$.

RESULTS: No discordance between colony counts or clinical interpretation of results occurred in the first part of the study. In the second part of the study, 263 urine specimens (16.9\%) showed differences in growth of one or several uropathogens. No statistically significant difference was observed in the colony counts using the Wilcoxon Matched Pairs Signed Rank test. The $\mathrm{P}$ value for the test was 0.6848 . There was no discordance 
in species of pathogen isolated. Two $(0.76 \%)$ discordant colony counts $(>1$ $\log _{10}$ difference) occurred, both with $P$. aeruginosa as the implicated pathogen and both with the CHROMagar ${ }^{\mathrm{TM}}$ reporting lower counts $\left(2 \times 10^{6} \mathrm{cfu} / \mathrm{L}\right.$ and $\left.1 \times 10^{6} \mathrm{cfu} / \mathrm{L}\right)$ than the split plates $\left(4 \times 10^{7} \mathrm{cfu} / \mathrm{L}\right.$ and $1 \times 10^{8} \mathrm{cfu} / \mathrm{L}$, respectively). In both cases, the isolate occurred in pure culture and in the presence of reported symptoms and thus would have been reported regardless of the low colony count.

CONCLUSION: Automated streaking of CHROMagar ${ }^{\mathrm{TM}}$ plates did not significantly alter quantitative colony counts or interpretation when compared to standard manual streaking. We support the use of automated inoculation as it reduces repetitive workload for staff.

\section{Student Poster Presentations Thursday, February 28 Grand Ballroom (Exhibit Hall)}

\section{SP1}

DEMOGRAPHIC AND REGIONAL FACTORS PREDICTING ANTIMICROBIAL SUSCEPTIBILITY IN ESCHERICHIA COLI FROM CANADIAN INTENSIVE CARE UNITS

P Lagacé-Wiens ${ }^{1 *}$, M Decorby ${ }^{1}$, P Baudry ${ }^{1}$, D Hoban ${ }^{1,2}$, J Karlowsky ${ }^{1,2}$, G Zhanel1,2

${ }^{1}$ University of Manitoba, Department of Medical Microbiology and Infectious Diseases; ${ }^{2}$ Clinical Microbiology, Health Sciences

Centre, Winnipeg, MB

OBJECTIVES: Escherichia coli resistance to antimicrobials varies according to many factors. We studied $E$. coli isolates from Canadian intensive care units (ICUs) to determine the distribution and demographics associated with antimicrobial resistance in this population.

METHODS: The Canadian National Intensive Care Unit (CAN-ICU) study characterized pathogens isolated in Canadian ICUs from July 2005 to June 2006. E. coli susceptibility to 11 antimicrobials was determined and a multivariate logistic regression model was designed to determine if province of origin, gender, specimen source, isolation from a sterile site, isolation from urine, and age $>30$ were significantly associated with susceptibility to the tested antimicrobials, to multi-drug resistance, or pansusceptibility.

RESULTS: 536 E. coli isolates, representing $12.6 \%$ of all isolates collected in the CAN-ICU study were studied. Susceptibilities were highest for meropenem and tigecycline (100\%), amikacin (99.8\%), cefepime (98.3\%), and piperacillin-tazobactam $(97.2 \%)$ and lowest for cefazolin (71.4\%), trimethoprim-sulfamethoxazole $(75.2 \%)$, and the fluoroquinolones (ciprofloxacin, $78.1 \%$; levofloxacin, $78.7 \%$ ). In the multivariate model, fluoroquinolone resistance was lowest in patients under 30 years and those with a urinary tract isolate. Cefazolin and ceftriaxone susceptibility was lowest in Nova Scotia and highest in New Brunswick. Pan-susceptibility was lowest in Nova Scotia and British Columbia and highest in New Brunswick. Isolation from a sterile site was associated with trimethoprim-sulfamethoxazole, piperacillin-tazobactam and multi-drug resistance. CONCLUSIONS: E. coli antimicrobial susceptibility varies across Canadian ICUs. Age, province, and site of infection should be considered when prescribing empiric antimicrobial therapy. Fluoroquinolones, cefazolin, and sulfonamides should be avoided due to low susceptibilities. Local antimicrobial prescribing practices, in particular the liberal use of fluoroquinolones and cephalosporins, and inadequate infection control practices are likely reducing susceptibility rates.

\section{SP2}

EVALUATION OF ETEST COMPARED TO THE REFERENCE BROTH MICRODILUTION ANTIFUNGAL SUSCEPTIBILITY TESTING OF CLINICAL ISOLATES OF CANDIDA SPP. AGAINST FOUR ANTIFUNGAL AGENTS

R Reyes ${ }^{1,2 *}$, M John ${ }^{1,2}$, R Lannigan 1,2, S Milburn ${ }^{1}$, M Witkowska ${ }^{3}$

${ }^{1}$ London Health Sciences Centre; ${ }^{2}$ Schulich School of Medicine, University of Western Ontario, London; ${ }^{3}$ Central Public Health Laboratory, Ontario Ministry of Health and Long-Term Care, Toronto, ON

OBJECTIVES: Invasive fungal infections are an increasing cause of nosocomial infections and increasing treatment failure due to antifungal resistance is ocurring. The Clinical and Laboratory Standards Institute (CLSI) M27-A2 reference broth microdilution method (BMD) is not used by most laboratories because of the low volume of susceptibility testing for Candida spp. Therefore, to provided timely results many laboratories perform susceptibility testing by the Etest method. The aim of this study was to evaluate the susceptibilities of Candida spp. to amphotericin B, ketoconazole, fluconazole, voriconazole and itraconazole. We evaluated Etest susceptibility results performed in our laboratory to those of BMD provided by the reference Public Health Laboratory in Toronto, ON.

METHODS: Antifungal susceptibility testing was performed on Candida spp. isolates from patient samples from 1999-2007. In vitro testing to amphotericin B, ketoconazole, fluconazole and itraconazole was performed by Etest and BMD. Susceptibility results to voriconazole were performed by BMD.

RESULTS: A total of 203 isolates of Candida spp. were tested, including 67 C. glabrata, 63 C. albicans, 37 C. parapsilosis, 17 C. tropicalis, 13 C. krusei, 3 C. dubliniensis, 2 C. guilliermondii and 1 C. lusitania species. The overall essential agreement between the Etest and BMD was calculated for amphotericin B, ketoconazole, fluconazole and itraconazole and was found to be $84.2 \%, 83.8 \%, 67.7 \%$ and $64.5 \%$ respectively. The overall categorical agreement for fluconazole and itraconzole was $78.2 \%$ and $52.3 \%$ respectively. Categorical agreement was not determined for amphotericin $\mathrm{B}$ and ketoconazole because CLSI has not established interpretive breakpoints for these antifungal agents. Of the Candida spp. tested, $100 \%$ of C. albicans, C. dubliniensis, C. guilliermondii, C. krusei, C. lusitania and C. parapsilosis were sensitive to voriconazole. $94 \%$ of tested C. glabrata and $83 \%$ of C. tropicalis were sensitive to voriconazole.

CONCLUSION: The essential agreement was lower with fluconazole and itraconzole because the MIC endpoint is determined by subjective assessment of $80 \%$ inhibition of growth. Overall, Etest compared favourably with the CLSI reference BMD method for determining the susceptibility of Candida spp.

\section{SP3}

STREPTOCOCCUS PNEUMONIAE IN CANADIAN INTENSIVE CARE UNITS: PREVALENCE AND ANTIBIOTIC RESISTANCE RESULTS OBTAINED DURING 2005 AND 2006 CANADIAN INTENSIVE CARE UNIT SURVEILLANCE STUDY (CAN-ICU) GG Zhanel ${ }^{1,2}$, DJ Hoban ${ }^{1,2}$, M Decorby ${ }^{1}$, K Nichol' ${ }^{2}$, AK Wierzbowski ${ }^{1 *}$

${ }^{1}$ University of Manitoba; ${ }^{2}$ Health Sciences Centre, Winnipeg, MB BACKGROUND: The respiratory tract is the most common site of infections in intensive care units (ICUs), with high mortality rates from nosocomial/ventilatory acquired pneumonia. The objective of this study was to assess prevalence and antibiotic susceptibility of $S$. pneumoniae among respiratory tract isolates obtained from across intensive care units in Canada. METHODS: 4180 study isolates were obtained as part of the Canadian National Intensive $\underline{\text { Care }}$ Unit (CAN-ICU) Study. The CAN-ICU study included 19 medical centres from all regions of Canada with active ICUs. From September 2005 to June 2006, inclusive, each centre collected a maximum of 300 consecutive pathogens isolated from blood, urine, tissue/wound, and respiratory specimens of ICU patients. Isolates were shipped to the reference laboratory (Health Sciences Centre, Winnipeg, Canada) on Amies charcoal swabs, subcultured onto appropriate media, and stocked in skim milk at $-80^{\circ} \mathrm{C}$ until minimum 
inhibitory concentration (MIC) testing was performed. MIC testing was carried out according to CLSI approved broth microdilution method. RESULTS: Out of a total of 2292 respiratory isolates obtained, 198 (8.6\%) were identified as S. pneumoniae. 65.1\% (129/198) of S. pneumoniae isolates were identified in patients 18 to $64 \mathrm{yrs}, 22.7 \%(45 / 198)$ in patients $\geq 65$ and $12.1 \%$ (24) in patients $\leq 17$ yrs. $38.9 \%$ (77/198) of isolates were identified in female patients and $61.1 \%(121 / 198)$ were from male patients. All isolates were susceptible to cefepime, ceftriaxone, linezolid and vancomycin. Resistance rates to other commonly used agents were: clarithromycin, $16.4 \%$; trimethoprim/sulfamethoxazole, $7.9 \%$; clindamycin, 6.4\%; ciprofloxacin, 2.6\%; levofloxacin, 2.1\%; and moxifloxacin, $1.6 \%$.

CONCLUSION: S. pneumoniae was the $4^{\text {th }}$ most common respiratory tract pathogen, responsible for $8.6 \%$ of respiratory tract infections in Canadian ICUs. Resistance rates were $0 \%$ with ceftriaxone, linezolid and vancomycin, $2.1 \%$ with levofloxacin and $16.4 \%$ with clarithromycin.

\section{SP4}

IN VITRO ACTIVITY OF TIGECYCLINE AGAINST COMMUNITY AND HOSPITAL STRAINS OF METHICILLIN RESISTANT STAPHYLOCOCCUS AUREUS (MRSA) AT PROVIDENCE HEALTH CARE (PHC)

A Stefanovic $^{1 *}$, S Champagne ${ }^{2,3}$, M Hull ${ }^{1}$, R Sebastian ${ }^{3}$, M Romney ${ }^{2,3}$

${ }^{1}$ University of British Columbia, Department of Medicine, Division of Infectious Diseases; ${ }^{2}$ PHC, Department of Pathology and Laboratory Medicine, Division of Medical Microbiology; ${ }^{3} \mathrm{PHC}$, Infection Prevention and Control, Vancouver, BC

OBJECTIVE: Tigecycline is a novel glycylcycline antibiotic with broadspectrum antimicrobial activity, including MRSA. At Providence Health Care, CMRSA-10 is the predominant community-associated strain and CMRSA-6 is the established hospital-associated strain. The purpose of this study was to determine the in vitro activity of tigecycline against MRSA isolates collected in a variety of settings in downtown Vancouver. A secondary objective was to compare the activity of tigecycline against CMRSA-10 and CMRSA- 6 types.

METHODS: Representative isolates of clinical specimens (blood, sputum, bronchoscopy washings, wound, throat, urine) and surveillance swabs (nares, wounds) from the period of January to November 2006 were selected for susceptibility testing. Molecular testing by pulsed-field gel electrophoresis (PFGE), for Panton-Valentine Leukocidin (PVL) and mecA genes was previously performed on these isolates. Disk diffusion testing for tigecycline was conducted by the Kirby-Bauer (KB) method. Tigecycline Minimum Inhibitory Concentration (MIC) values were determined using standard Etest ${ }^{\circledR}$ methodology and recorded without previous knowledge of molecular results. $\mathrm{MIC}_{50}$ and $\mathrm{MIC}_{90}$ were calculated and further analyzed according to PFGE type.

RESULTS: 200 MRSA isolates were tested by both KB and MIC method, of which $100 \%$ were found to be susceptible to tigecycline: mean zone diameter $25.7 \mathrm{~mm}$; range $20-35 \mathrm{~mm} ; \mathrm{MIC}_{50}=0.125 \mathrm{mg} / \mathrm{L}$ and $\mathrm{MIC}_{90}$ $=0.380 \mathrm{mg} / \mathrm{L}$. $\mathrm{MIC}_{50}$ and $\mathrm{MIC}_{90}$ values were twice as high for predominant hospital type (CMRSA-6; $\mathrm{MIC}_{50}=0.250 \mathrm{mg} / \mathrm{L}$ and $\mathrm{MIC}_{90}=0.500 \mathrm{mg} / \mathrm{L}$ ) compared to the main community type (CMRSA-10; $\mathrm{MIC}_{50}=0.125 \mathrm{mg} / \mathrm{L}$ and $\left.\mathrm{MIC}_{90}=0.250 \mathrm{mg} / \mathrm{L}\right)\left(\mathrm{p}<0.01\right.$ for $\left.\mathrm{MIC}_{50}\right)$.

CONCLUSION: Using two different susceptibility methods, tigecycline appears to have excellent in vitro activity against MRSA. MIC 50 and $\mathrm{MIC}_{90}$ values may vary according to molecular strain type, with community strains appearing more susceptible to tigecycline than hospital strains in downtown Vancouver. Further studies are needed to evaluate clinical implications of this in vitro phenomenon.

\section{SP5}

EPIDEMIOLOGY OF INVASIVE MENINGOCOCCAL DISEASE WITH DECREASED PENICILLIN SUSCEPTIBILITY IN ONTARIO 2000-2006

E Brown $^{1 *}$, D Fisman ${ }^{1,3}$, S Brown ${ }^{1}$, P Rawte ${ }^{1}$, S Dolman²,

F Jamieson ${ }^{1}$

${ }^{1}$ Ontario Central Public Health Laboratory; ${ }^{2}$ Ontario Public Health Branch; ${ }^{3}$ Hospital for Sick Children, Toronto, ON

BACKGROUND: Neisseria meningitidis is an important cause of meningitis and septicaemia (i.e., invasive meningococcal disease (IMD)), but has been slow to acquire resistance to penicillin and other antimicrobials. We previously reported an increase in the incidence of IMD strains with decreased susceptibility to penicillin (IMD-DSP) in Ontario between 1997 and 2000. Our current objectives are to evaluate trends in IMD-DSP; to identify case-level predictors of IMD-DSP; and to evaluate the effects of DSP on survival.

METHODS: All IMD isolates in Ontario are submitted to the Central Public Health Laboratory for confirmation and serogroup evaluation. Penicillin susceptibility testing was performed using the agar dilution method; isolates were considered to be IMD-DSP if penicillin MIC was $\geq 0.125 \mathrm{ug} / \mathrm{ml}$. Case outcome was determined through linkage to public health case investigation files. Temporal trends were evaluated using multivariable Poisson regression models, and correlates of diminished susceptibility and fatal outcome were evaluated with multivariable logistic regression models.

RESULTS: 389 unique IMD isolates were available for analysis. The overall rate of IMD in Ontario from 2000 to 2006 was estimated to be 4.6 cases per million population ( $95 \%$ CI 4.1 to 5.0 ) with distinct wintertime seasonality, and a downward trend in case occurrence that was strongest for vaccine-preventable serogroup C disease (yearly RR 0.82 , 95\% CI 0.75 to 0.91). 79 isolates (20\%) were IMD-DSP. Even after adjustment for overall trends in IMD, there was no trend in either the incidence of IMD-DSP (yearly RR $1.06,95 \%$ CI 0.96 to 1.17 ) or in average log-MIC to penicillin in IMD strains (yearly change $-0.01,95 \% \mathrm{CI}-0.04$ to 0.02 ). IMD-DSP was strongly associated with serogroup Y (OR 6.3, 95\% CI 3.6 to 11.1 ) and W-135 (OR 8.2, $95 \%$ to 4.0 to 16.7 ), and was highest in incidence outside Greater Toronto (RR 3.1, 95\% CI 1.9 to 5.1 ). There was no association between IMD-DSP and mortality (OR 0.6, 95\% CI 0.3 to 1.3 ), but IMD due to serogroup $\mathrm{C}$ was associated with a marked increase in risk of mortality (OR 2.47, 95\% CI 1.34 to 4.56 ).

CONCLUSIONS: In contrast to trends in the late 1990s, the incidence of IMD-DSP was stable in Ontario between 2000 and 2006. There was no association between IMD-DSP and risk of death in this study; in Ontario serogroup rather than penicillin MIC appears to be the microbiological parameter most predictive of mortality.

\section{SP6}

ANTIBACTERIAL EVALUATION AND PHYTOCHEMICAL SCREENING OF ANETHUM GRAVEOLENS, FOENICULUM VULGARE, TRACHYSPERMUM AMMI, AMOMUM SUBULATUM AND ELETTARIA CARDAMOMUM

\section{GJ Kaur*, DS Arora}

Guru Nanak Dev University, Amritsar, Punjab, India

OBJECTIVE: Evaluation of natural products for safe and effective antimicrobial agents is one of the scientific strategies to combat the ever increasing menace of resistant pathogens. Plants have been in use for the treatment of infectious diseases since times immemorial but the validity of these remedies remains questionable due to lack of any scientific evidence for their effectiveness. The last 20-25 years have witnessed a renewed interest towards folkloric remedies to work out novel chemotherapeutics and an impressive number of modern drugs have already been developed from plants. The objective of this study was to provide a scientific proof for traditional uses of these plants and to expand the antibacterial spectrum from plants.

METHODS: Aqueous (ambient, hot and boiling water) and organic (hexane, ethyl acetate, acetone and ethanol) extracts of five medicinal plants viz. Anethum graveolens, Foeniculum vulgare, Trachyspermum ammi, 
Amomum subulatum and Elettaria cardamomum were assessed for their antibacterial potential against eleven reference strains of bacteria using agar diffusion assay, minimum inhibitory concentration (MIC) and viable cell count (VCC) studies. Antibacterial activity of these plant extracts was compared with commonly employed antibiotics by disc diffusion assay. All the plants were screened for their phytoconstituents viz. alkaloids, flavonoids, tannins, saponins and cardiac glycosides using chemical treatment methods and thin layer chromatography. The different values have been expressed as mean \pm standard deviation. P values $<0.05$ were considered as significant

RESULTS: Aqueous extracts prepared in hot water gave better inhibitory zones ranging from $12-28 \mathrm{~mm}$ as compared to extracts prepared at ambient temperature and boiling water whereas acetone was the best with zone size 9-34 mm among different organic solvents used for extraction. Gram-positive bacteria (Staphylococcus aureus and Enterococcus faecalis) showed greater sensitivity as compared to Gram-negative bacteria. Minimum inhibitory concentration (MIC) ranged from 2-8\% (aqueous extracts) and 0.5-1.5\% (acetone extracts) while viable cell count (VCC) studies confirmed their bactericidal nature. Statistical analysis showed better/equally effective antibacterial activity of the plant extracts as compared to some standard antibiotics. Qualitative phytochemical screening showed the presence of alkaloids, flavonoids, tannins, saponins and cardiac glycosides while quantitative determination revealed $1.1-4.23 \%$ alkaloids, $3.5-15.8 \%$ flavonoids and $0.5-0.7 \%$ saponins in these plant extracts.

CONCLUSION: Better/equal efficacy of these plant extracts as compared to standard antibiotics validates their traditional uses thereby providing a scientific basis to homemade remedies. The presence of different phytochemicals in the plants supports their antibacterial potential. Isolation and purification of such compounds may yield significant antibacterial agents from these plants.

\section{SP7 \\ MULTI-DRUG RESISTANT EXTENDED-SPECTRUM $\beta$ - LACTAMASE PRODUCING ESCHERICHIA COLI FROM PATIENTS IN CANADIAN HOSPITALS: RESULTS FROM CAN-WARD 2007}

P Baudry ${ }^{*}$, M Decorby ${ }^{1}$, K Nichol ${ }^{2}$, P Lagace-Wiens ${ }^{1}$, GG Zhanel ${ }^{1}$, DJ Hoban ${ }^{1,2}$

${ }^{1}$ University of Manitoba; ${ }^{2}$ Health Sciences Centre, Winnipeg, MB

OBJECTIVE: Extended-Spectrum $\beta$-lactamase (ESBL) producing E. coli have become a growing cause of community- and nosocomial- acquired infections. ESBLs are highly mobile enzymes that confer resistance to $3^{\text {rd }}$ generation cephalosporins by hydrolysis and have been associated with resistance genes to several other unrelated antimicrobial classes. The purpose of this study was to assess the prevalence and to molecularly characterize multi-drug resistant (MDR: resistance to $\geq 3$ different antibiotic classes) ESBL producing E. coli from patients affiliated with Canadian hospitals.

METHODS: 1227 E. coli isolates were collected as part of the CANWARD Surveillance Study from January 2007 to present. Susceptibility testing was performed by broth microdilution and putative ESBL isolates were screened by the CLSI disk diffusion method. PCR and DNA sequencing were used to detect $b l a_{\mathrm{TEM}}, b l a_{\mathrm{SHV}}, b l a_{\mathrm{OXA}}$ and $b l a_{\mathrm{CTX}-\mathrm{M}}$ genes. Strains were sub-typed using pulsed-field gel electrophoresis (PFGE).

RESULTS: 34 (2.8\%) of 1227 E. coli were ESBL producers. Of these, 32 $(94.2 \%)$ were fluoroquinolone (ciprofloxacin $\geq 4 \mathrm{ug} / \mathrm{mL}$ ) resistant, 24 (70.6\%) were TMP/SMX ( $\geq 4 / 76 \mathrm{ug} / \mathrm{mL})$ resistant and 18 (52.9\%) were gentamicin ( $\geq 16 \mathrm{ug} / \mathrm{mL})$ resistant. All 34 (100\%) ESBL producing E. coli were identified as MDR. PCR and sequencing revealed $15(44.1 \%)$ bla $a_{\text {CTX-M-15 }}, 13$ (38.2\%) bla $a_{\text {CTX-M-14 }}, 3$ (8.8\%) bla OXA and 1 (2.9\%) each of bla $a_{\text {CTX-M-24, }}$ bla $a_{\text {CTX-M-27 }}$ and bla $a_{\text {CTX-M-65. }} 18$ (52.9\%) of the ESBL producing E. coli also carried bla $a_{\mathrm{TEM}-1}$ and 11 of 15 (73.3\%) of CTX-M-15 ESBLs also carried bla OXA-30. No bla $a_{\text {SHV }}$ ESBLs were found. Genetically related ( $>80 \%$ homology) and unrelated groups of ESBLs were observed. Patient demographics revealed a mean age of 55 years with a range of 23 to 89 years, with females representing $50 \%$ (17) of patients and males representing $50 \%$ (17) of patients. $16(47.1 \%), 15(44.1 \%), 2(5.8 \%)$ and 1
(2.9\%) of the ESBL producing strains were isolated from urine, blood, respiratory tract and wounds, respectively.

CONCLUSION: The prevalence of ESBL producing E. coli in hospital affiliated patients across Canada is $2.8 \%$. bla $a_{\text {CTX-M-15 }}$ and $b l a_{\text {CTX-M-14 }}$ are the predominant genotypes carried by ESBL producing E. coli. Genetically related and unrelated MDR ESBL producing E. coli are present in Canada.

\section{SP8}

CROSSING THE THRESHOLD: RESISTANCE TO FIRST-LINE ANTIBIOTICS IN UROPATHOGENIC ESCHERICHIA COLI A Chau ${ }^{1,2 *}$, D Purych ${ }^{3,4}$, L Vrbova ${ }^{1,2}$, D Patrick ${ }^{1,2}$

${ }^{1} \mathrm{BC}$ Centre for Disease Control; ${ }^{2}$ University of British Columbia, Vancouver; ${ }^{3}$ Fraser Health Authority; ${ }^{4} \mathrm{BC}$ Biomedical Laboratories, Surrey, BC

OBJECTIVE: An important uropathogen, Escherichia coli, is responsible for up to $90 \%$ of uncomplicated urinary tract infections. Current recommendations for first-line antibiotic therapy include trimethoprim-sulfamethoxazole (TMP-SMX), fluoroquinolones, and nitrofurantoin. The aim of this paper is to analyze antimicrobial resistance (AMR) trends in connection with antibiotic consumption rates and discuss possible implications for therapy.

METHODS: E. coli susceptibility data between 1998 and 2007 were obtained from BC Biomedical Laboratories. Antibiotic consumption rates were provided by IMS Health and BC PharmaNet and were measured in defined daily doses (DDD). Using SPSS 14.0, the two-sided Spearman Rank test was performed to determine correlation between AMR trends and antimicrobial consumption with a twelve month lag time.

RESULTS: Percent of isolates resistant to ciprofloxacin and TMP-SMX steadily increased from years 1998 to 2007 ( $\mathrm{p}<0.01)$. Ciprofloxacin nonsusceptibility among E. coli isolates increased 10 -fold to $22.0 \%$ in 2007 and was significantly correlated to fluoroquinolone consumption $(\mathrm{p}<0.01)$. $23.8 \%$ of isolates were non-susceptible to TMP-SMX in 2007. Percent of isolates non-susceptible to nitrofurantoin remained stable and low $(\leq 4 \%)$. The increasing trend in nitrofurantoin consumption was not significantly associated with decreasing nitrofurantoin susceptibility.

CONCLUSION: Nitrofurantoin remains a good treatment option due to high susceptibility rates and low association between consumption and non-susceptibility. However, the continual increase and high numbers of isolates non-susceptible to ciprofloxacin and TMP-SMX indicate the need to consider less frequent use of these existing first-line agents for treating uncomplicated urinary tract infections.

\section{SP9}

\section{ACTIVITY OF LIPOSOMAL ANTIBIOTICS IN SPUTUM OF CYSTIC FIBROSIS PATIENTS}

M Alipour ${ }^{1 *}$, ZE Suntres ${ }^{2}$, M Halwani ${ }^{1}$, A Kumar ${ }^{1}$, A Omri ${ }^{1}$

${ }^{1}$ Laurentian University, Sudbury; ${ }^{2}$ Lakehead University, Thunder Bay, ON

INTRODUCTION: Chronic infections caused by opportunistic pathogens in the lower respiratory tract have become the major cause of morbidity and mortality in Cystic Fibrosis (CF) patients. Sputum formation in the airways shields pathogens from antibiotic therapy and this resistance compels the need for the development of novel therapeutic concepts. This study compares the effectiveness of antibiotics (tobramycin and polymyxin B) in conventional or liposome-encapsulated form in reducing bacterial count in CF sputum. Also, due to the mucus structure of sputum and high contents of proteases, DNA, and ions, the effect of sputum on antibiotic penetration was studied by examining the antibiotic inhibition of the formulations in autoclaved sputum "spiked" with resistant strains of P. aeruginosa.

METHOD: Pooled sputum containing P. aeruginosa strains from CF patients was diluted $1: 10(\mathrm{w} / \mathrm{v})$ in nutrient broth. Tobramycin or polymyxin B liposomes were prepared from a mixture of dimyristoylphosphatidylcholine or dipalmitoylphosphatidylcholine and cholesterol, respectively. Serial-diluted antibiotic formulations were added to nutrientdiluted sputum and incubated at $37^{\circ} \mathrm{C}$ for 18 hours. The Minimum Inhibitory Concentrations (MIC) were measured in diluted sputum 
containing susceptible or resistant strains of $P$. aeruginosa, in order to measure the antimicrobial activity of the formulations.

RESULTS: None of the formulations used eradicated the bacterial strains. However, the MIC for liposomal tobramycin $(128 \mathrm{mg} / \mathrm{L})$ was better than that observed for free tobramycin $(512 \mathrm{mg} / \mathrm{L})$ and liposome-encapsulated polymyxin B $(8 \mathrm{mg} / \mathrm{L})$ was better than that observed for free polymyxin B $(32 \mathrm{mg} / \mathrm{L})$. "Spiking" sputum with resistant strains of P. aeruginosa reduced the activity of liposomal tobramycin and liposomal polymyxin B by 4-fold, while still displaying considerably lower MICs compared to their conventional forms. An 8- and 2-fold increase in MICs was noticed in susceptible $P$. aeruginosa strains when treated with either free or liposomal formulations.

CONCLUSION: The decrease in bacterial count and MICs are evidence to suggest that the liposomal antibiotics are more effective than the conventional form in presence of sputum.

\section{SP10}

\section{ANTIMICROBIAL SUSCEPTIBILITY OF $\beta$-HAEMOLYTIC STREPTOCOCCI IN DOWNTOWN VANCOUVER,} 2004-2007

K Tan ${ }^{1 *}$, M Hull2,1, M Romney ${ }^{2,1}$, S Champagne ${ }^{2,1}$

${ }^{1}$ University of British Columbia; 2Providence Health Care,

Vancouver, BC

OBJECTIVES: $\beta$-haemolytic streptococci (BHS) remain universally susceptible to penicillin. As such, susceptibility testing for other antimicrobials is not routinely performed in most laboratories. Despite this, research studies have demonstrated the development of resistance to non- $\beta$-lactam antimicrobials, particularly macrolides and clindamycin. The purpose of this study was to determine susceptibility of Groups A, B, C and G (GAS, GBS, GCS, GGS) BHS to alternative antibiotics: erythromycin, clindamycin, tetracycline and moxifloxacin.

METHODS: Susceptibility testing was performed on isolates from blood, sterile body sites and wounds collected between January 2004 and August 2007 at Providence Health Care, Vancouver, BC. Susceptibility was determined using Kirby-Bauer methodology, and the D-test was used to characterize $\mathrm{MLS}_{\mathrm{B}}$ phenotype. Erythromycin, clindamycin, and tetracycline susceptibilities were based on the CLSI standards, while moxifloxacin was determined using French guidelines (Société Française de Microbiologie, 2007).

RESULTS: A total of 245 isolates were analyzed: 163 GAS (67\%), 45 GBS (18\%), 5 GCS (2\%) and 32 GGS (13\%). Of the 245 isolates, 195 $(80 \%)$ were from blood and sterile body sites. Overall, susceptibilities to erythromycin, clindamycin, tetracycline and moxifloxacin were $73 \%$, $73 \%, 47 \%$ and $93 \%$, respectively. Clindamycin resistance was predominantly inducible in nature. GAS was generally more susceptible than other BHS with $79 \%$ susceptible to clindamycin and $60 \%$ susceptible to tetracycline. In contrast, GGS showed 53\% susceptibility to clindamycin and $9 \%$ to tetracycline. Susceptibility profiles were similar in isolates from sterile and non-sterile sites.

CONCLUSIONS: These data demonstrate high rates of nonsusceptibility of BHS to non- $\beta$-lactam antibiotics. Empiric treatment of soft tissue infections with tetracyclines should be discouraged in view of increased rate of resistance. Enhanced susceptibility testing should be considered on selected clinical isolates. At present, moxifloxacin resistance appears low; however, continued surveillance is required for long term evaluation.

\section{SP11}

CIPROFLOXACIN RESISTANCE AMONG UROSEPTIC PATIENTS PRESENTING TO THE EMERGENCY DEPARTMENT AT A MAJOR TERTIARY CARE HOSPITAL C Lowe $^{1 *}$, I Momenkhan ${ }^{2}$, R Reynolds ${ }^{2}$, D McKnight ${ }^{3}$, T Lee ${ }^{3}$, D Roscoe ${ }^{4}$, P Doyle 4

${ }^{1}$ University of British Columbia; ${ }^{2}$ Division of Infectious Diseases, Vancouver General Hospital; ${ }^{3}$ Department of Emergency Medicine, Vancouver General Hospital; ${ }^{4}$ Division of Medical Microbiology and Infection Control, Vancouver General Hospital, Vancouver, BC OBJECTIVE: Fluoroquinolone resistance among Escherichia coli isolated from outpatient urine has been reported at 5.5\% in 2004 from across North America (NAUTICA study). According to the current Urosepsis Protocol at our hospital, Ciprofloxacin or Ampicillin/Gentamicin are considered first-line empiric therapy. In light of concerns regarding increasing antibiotic resistance, the objective of this study was to determine the appropriateness of Ciprofloxacin as a first-line agent in uroseptic patients presenting to the Emergency Department (ED).

METHODS: Patients with urosepsis presenting to VED were identified utilizing the Laboratory database. Over a 2-year period (October 1, 2005-September 30, 2007), positive blood cultures with Escherichia coli, Klebsiella pneumoniae, Proteus mirabilis, Enterobacter cloacae or Psendomonas aeruginosa were identified and were correlated with urine cultures from the same day. Urosepsis was defined as isolation of the same Gram negative organism on blood and urine cultures on the same day. A chart review was also conducted to determine possible risk factors for Ciprofloxacin-resistant organisms, including past visits to our hospital (within the last 4 weeks), and past microbiology cultures (Gram negative bacilli cultured in urine in the previous 3 months).

RESULTS: During the 2-year period, 379 blood cultures were identified, and from that a total of 150 patients with Gram negative urosepsis were identified, $87 \%$ of which were E. coli. Of these cases, 20.7\% (30 E. coli and $1 \mathrm{~K}$. pneumoniae) were found to be Ciprofloxacin resistant and $9.3 \%$ (14 E. coli) were resistant to Gentamicin. Among Ciprofloxacin resistant E. coli, 43.3\% were also resistant to Ampicillin and Gentamicin. Ciprofloxacin resistance was associated with recent visits to our hospital $(\mathrm{p}<0.027)$ and previous positive cultures $(\mathrm{p}<0.034)$.

CONCLUSION: An elevated rate of resistance to Ciprofloxacin in Gram negative uropathogens was discovered in uroseptic patients presenting to the ED. Risk factors for developing resistance were past visits to VGH within the last 4 weeks and positive urine cultures within the last 3 months. Alternative therapy for patients with urosepsis should be considered, particularly in those presenting with risk factors for Ciprofloxacin resistance.

\section{SP12}

BROTH MICRODILUTION DETECTION OF INDUCIBLE CLINDAMYCIN RESISTANCE IN METHICILLIN-RESISTANT STAPHYLOCOCCUS AUREUS (MRSA)

E Sy $^{2 *}$, C Watt ${ }^{1}$, L Louie $^{1}$, AE Simor ${ }^{1,2}$

${ }^{1}$ Sunnybrook Health Sciences Centre, Toronto, $\mathrm{ON}$; ${ }^{2}$ University of Toronto, Toronto, ON

OBJECTIVE: Detection of inducible clindamycin resistance (macrolide, lincosamide, streptogramin $B ; M_{1} B_{i}$ ) in MRSA has traditionally been detected using the CLSI recommended double-disk diffusion test (D-test). We wished to determine if a combination of erythromycin (ER) and clindamycin (CL) could be used in a microdilution format for detection of $\mathrm{MLSB}_{\mathrm{i}}$ in MRSA.

MATERIALS AND METHODS: Limited checkerboard testing with combinations of ER and CL were tested. Broth microdilution (BMDIL) with ER and CL, separately and in combination were performed on a selection of 358 strains of Canadian isolates of MRSA (148 D-test positive strains, 150 D-test negative strains, 33 ER/CL resistant isolates, and 27 ER/CL susceptible strains) and compared with conventional D-test and detection of $\mathrm{MLSB}_{\mathrm{r}}$ and $\mathrm{MSB}_{\mathrm{r}}$ using PCR for the ermA, ermC, and msrA resistance determinants.

RESULTS: A combination of $1.5 / 8 \mu \mathrm{g} / \mathrm{ml} \mathrm{CL/ER}$ was able to detect all 148 D-test positive isolates while other concentrations of CL/ER were less effective. PCR detection of $m s r A$ and ermA/C positive strains could distinguish MSB resistance from $\mathrm{MLSB}_{\mathrm{i}}$. The ermA gene was most frequently found in the hospital-associated CMRSA-2 (USA100) clone while msrA was almost exclusively found in strains belonging to the CMRSA-10 clone (USA300).

CONCLUSIONS: A combination of $1.5 / 8 \mu \mathrm{g} / \mathrm{ml}$ CL/ER in a BMDIL format was able to reliably detect inducible CL resistance in strains of MRSA. Further testing with a larger number of isolates would be required to validate the method. The development and incorporation of a $1.5 / 8 \mu \mathrm{g} / \mathrm{ml}$ ERY/CLD well in commercially available automated systems would possibly obviate the need for the more cumbersome double diffusion D-test. 


\section{SP13}

A CASE OF NON-HEALING CUTANEOUS ULCERS

M Alawi*, C Kraeker, N Singhal

McMaster, Hamilton, ON

BACKGROUND: Cutaneous cryptococcosis can be a primary manifestation due to direct inoculation from injury or environmental contamination of pre-existing wounds or, more commonly, secondary to disseminated disease, usually in an immunocompromised host. Manifestations of both are protean; including papules, pustules, ulcers, nodules, subcutaneous abscesses or sinuses and diagnosis requires a high index of suspicion.

OBJECTIVE: To illustrate the importance of considering fungal infections such as Cryptococcus in patients with non-healing ulcers.

Case Scenario: An 80-year-old woman with pulmonary fibrosis, diabetes, coronary artery disease and congestive heart failure presented with multiple ulcers, located under the left breast, on the medial right thigh and on both lower extremities, of approximately 7 months duration. She had received IV antibiotics and various dressings with minimal response. The ulcers varied in size and appearance and felt to be multifactorial in nature. The patient was afebrile, and did not recall any trauma or history of cellulitis. She was seen by plastic surgery for debridement. Tissue cultures from her left breast grew Cryptococcus neoformans. A lumbar puncture was performed and showed normal spinal fluid analysis, a negative latex agglutination test and no growth on fungal culture. Her chest radiograph was non-revealing. Serum cryptococcal antigen test was positive with a titer of 1:32. The patient was started on fluconazole with significant improvement of her cellulitis and ulcers. The patient later developed worsening congestive heart failure and died.

CONCLUSION: Failure to consider cryptococcal infection in patients presenting with chronic non-healing cutaneous ulcers in the appropriate setting may be a pitfall. A lack of response to appropriate antibiotic and wound care should raise suspicion and prompt a more extensive workup. Individuals with cutaneous Cryptococcus should be evaluated for evidence of disseminated disease.

\section{SP14}

INTRACRANIAL TUBERCULOMA MISDIAGNOSED AS ENPLAQUE MENINGIOMA AND NEUROSARCOIDOSIS

\section{J Kanji*, P El-Helou}

McMaster University, Hamilton, ON

OBJECTIVE: Intracranial tuberculomas are rare in the Western World, comprising $0.2 \%$ of intracranial mass lesions. The differential diagnosis of such a mass lesion is quite broad encompassing meningioma, lymphoma, arterio-venous malformation, metastatic tumor, and neurosarcoidosis. The authors present the case of a woman with a delayed diagnosis of intracranial tuberculoma after being treated for en-plaque meningioma and neurosarcoidosis and pertinent literature review of the topic.

CLINICAL PRESENTATION: A 62-year old female with a prior diagnosis of neurosarcoidosis made 2 years ago presented with intermittent fever, headache, and confusion. She was found to have an abnormal neurological exam and suffered from several observed generalized seizures. Imaging revealed two new intracranial hypodense lesions with hydrocephalus. Cerebrospinal fluid collected from a lumbar puncture was sent for a diagnostic evaluation.

CONCLUSION: The diagnosis of intracranial tuberculoma is quite challenging and requires a high degree of suspicion. Despite the large differential, the diagnosis has unique features on imaging, histopathology, and interesting diagnostic laboratory test characteristics which are reviewed. However, since it is a treatable condition that can be exacerbated by therapy of other items in the differential, it should be completely ruled out before addressing alternative considerations.

\section{SP15}

CUTANEOUS LEISHMANIASIS IN FIVE CANADIAN SOLDIERS RETURNING FROM AFGHANISTAN TREATED WITH ORAL MILTEFOSINE

\section{O Larios*, Y Keynan, M Wiseman, E Rubinstein}

University of Manitoba, Winnipeg, MB

OBJECTIVE: Cutaneous leishmanisis (CL) is an important vector-borne disease globally with the potential to cause significant cicatrices. It is endemic to several Asian and Middle-Eastern countries where the rates of infection can be substantial. The 2 parasitic species known to cause $C L$ in Afghanistan are Leishmania major and Leishmania tropica. Traditionally CL is treated with intralesional antimonials or thermotherapy when small in size but when lesions are large or disfiguring, treatment with pentavalent antimonials such as sodium stibogluconate (SSG) or meglumine antimoniate (MGA) are used. SSG and MGA require 28 days of administration either intravenously or intramuscularly and have potentially fatal adverse effects. Systemic treatment with an oral drug such as miltefosine would provide greater compliance and favorable adverse event profile. A small published case series has shown equivalency between MGA and miltefosine for CL caused by $L$ major. We present a case series of 5 Canadian soldiers returning from Afghanistan with CL caused by $L$ tropica treated with miltefosine after not responding to oral fluconazole treatment. .

METHODS: Five Canadian soldiers were treated with oral miltefosine $2.5 \mathrm{mg} / \mathrm{kg}$ after presentation with cutaneous ulcerative lesions were biopsied and found to have amastigotes with PCR analysis revealing $L$ tropica. Treatment with miltefosine was started after the first patient showed lack of response to 6 weeks of oral fluconazole and failed to comply with SSG. RESULTS: All five patients' CL responded to treatment with oral miltefosine. One patient had to discontinue treatment earlier than the suggested 28 days due to nausea and vomiting but still had complete resolution of his lesion.

CONCLUSION: Oral miltefosine appears to be an efficacious treatment for CL due to L tropica acquired in Afghanistan and a randomized controlled trial should be instituted in light of these results. This is clinically important as Canadian physicians can expect to see more cases of CL from deployed military personnel to Afghanistan returning home and should become familiar with diagnosis and treatment of this disease.

\section{SP16}

VIRAL COMMUNITY ACQUIRED PNEUMONIA IN ADULTS: INCIDENCE, ORGANISMS, AND OUTCOMES

J Johnstone $^{1 *}$, S Majumdar ${ }^{1}$, J Fox ${ }^{2,3}$, T Marrie ${ }^{1}$

${ }^{1}$ University of Alberta, Edmonton; ${ }^{2}$ Provincial Laboratory for Public Health (Microbiology); ${ }^{3}$ University of Calgary, Calgary, AB

OBJECTIVES: Few studies have described virus-associated community acquired pneumonia (CAP) in adults since the advent of nucleic acid amplification tests (NATs) for diagnosis. Thus, we performed a prospective study in adults admitted to hospital with CAP, and sought to describe the incidence, etiology, presentation, and outcomes of viral CAP.

METHODS: From 2004-2006, consecutive immunocompetent adults admitted to 5 hospitals with CAP were enrolled and followed until discharge. Clinical data, blood and sputum cultures, serology, and nasopharyngeal swabs were obtained. Swabs were utilized for NATs targeted against respiratory syncytial virus (RSV), influenza viruses $A$ and B, parainfluenzaviruses 1-4, human metapneumovirus (hMPV), adenoviruses, coronaviruses (OC43, 229E and NL63), Chlamydophila pneumoniae, Mycoplasma pneumoniae and Legionella spp.

RESULTS: Over 20 months, 193 patients were included; mean age was 68 years, $51 \%$ were male and $48 \%$ had severe CAP (PSI class 4 or 5 ). Overall, $71(37 \%)$ of patients had a confirmed etiology. Twenty-five (13\%) patients had viral CAP, 46 (24\%) bacterial, the rest "unknown." Influenza A $(n=3)$, Influenza B $(n=4)$ and hMPV $(n=7)$ accounted for $56 \%$ of all viruses isolated among those with viral CAP; others included RSV $(n=5)$, coronavirus $(n=4)$, parainfluenza $(n=3)$ and adenovirus $(n=2)$. Of 46 bacterial cases of CAP, $16(35 \%)$ were caused by Streptococcus pneumoniae. Patients with viral CAP were older than those with bacterial and unknown CAP ( 73 years vs 61 years for bacteria $[p=0.007]$ vs 69 years for 
unknown $[\mathrm{p}=0.522])$ and more likely to have underlying cardiac disease ( $72 \%$ vs $30 \%$ for bacterial $[p=0.001]$ vs $38 \%$ for unknown $[p=0.002]$ ). There were almost no differences in presentation according to etiology. Median stay was 7 days, no patients went to ICU, and 7 (3\%) patients died - there were no significant differences in outcomes according to etiology. CONCLUSIONS: Viruses represented 13\% of CAP (35\% of confirmed etiologies) in our study. Influenza and hMPV caused most viral pneumonias. Viral CAP is common in adults and clinically indistinguishable from other etiologies. Our findings suggest routine diagnostic testing for viral etiologies may be warranted.

\section{SP17}

\section{PNEUMOCOCCAL EMPYEMA IN TORONTO, ONTARIO (1995-2006)}

T Lee $^{1 *}$, K Green ${ }^{2}$, A McGeer ${ }^{1,2}$, D Low ${ }^{2}$

${ }^{1}$ University of Toronto; ${ }^{2}$ Mount Sinai Hospital, Toronto, ON

OBJECTIVE: Recent studies have documented increases in the rate of pneumococcal empyema (PEMP). We examined trends in the occurrence of PEMP from 1995-2006.

METHODS: Population based surveillance for invasive pneumococcal disease (IPD) in residents of Toronto and Peel region, Ontario, Canada (pop=3.9M) has been on-going since 1995. In this study, PEMP includes cases with a positive pleural fluid culture and bacteremic cases with a clinical diagnosis of empyema. Clinical data was collected from patients and their physicians using a standardized collection form.

RESULTS: 4898 episodes of IPD have been identified, 154 (3.1\%) are PEMP. The incidence of empyema increased from an average of $0.28 / 100,000 / y$ in $1996-2001$ to $0.44 / 100,000 / y$ in $2002-6$, while the incidence of all IPD decreased from 11.6 to 9.9/100,000/y. Patients with PEMP were not significantly different from other IPD in age (median $56 y$ vs. $50 \mathrm{y})$, gender ( $55 \%$ vs. $59 \%$ male), or proportion that were healthcare acquired ( $4.5 \%$ vs. $4.0 \%$ ), but were more likely to have underlying lung disease or alcohol abuse ( $31 \%$ vs. $21 \%, P=.01,23 \%$ vs. $16 \%, P=.03$ ). They were also more likely to be failing outpatient oral antibiotics at presentation $(17.9 \%$ vs. $8.9 \%, \mathrm{p}=0.006)$. PEMP isolates were more likely to be of serotype 1 (6/136 PEMP vs. 27/4309 other, $\mathrm{P}<.001)$ and were less likely to be stains included in the pneumococcal conjugate vaccine (58/136 vs. 2456/4309, $\mathrm{p}=.001)$. They were also more likely to be resistant to macrolides (24/137 vs. 497/4330, p=.03), ciprofloxacin ( $7 / 137$ vs. 51/4330, $\mathrm{p} \leq 0.001)$, and levofloxacin $(6 / 137$ vs. $31 / 4330, \mathrm{P}<.001)$ but not penicillin (7/137 vs.168/4330) or moxifloxacin (1/137 vs. 9/4330). Those with empyema were more likely to require ICU admission (39\% vs. $23 \%$, $\mathrm{p}<.001)$ and mechanical ventilation ( $30 \%$ vs. $16 \%, \mathrm{p}<.001)$ but not more likely to die (20\% vs. $19 \%)$.

CONCLUSION: PEMP appears to be increasing despite a decrease in overall IPD. Serotype 1 is more likely to cause empyema than other IPD, although it does not make up the majority of cases. Empyema isolates were more likely to be resistant to macrolides, ciprofloxacin and levofloxacin but not penicillin or moxifloxacin as compared to other IPD. Empyema is associated with significant morbidity in terms of ICU admission and mechanical ventilation but not death as compared to other IPD.

\section{SP18 \\ BELIEFS, ATTITUDES AND OPINIONS REGARDING THE SHINGLES VACCINES AMONG CANADIANS OVER 60 \\ D Shaw*, S McNeil}

Dalhousie University, Halifax, NS

OBJECTIVE: Shingles is the cutaneous reactivation of infection by Varicella Zoster virus and is associated with significant morbidity and pain, with increasing frequency and intensity as people age. A new vaccine which reduces the occurrence of shingles and its complications will soon be available in Canada. In an effort to aid decision makers with information regarding potential demand for this vaccine and willingness of Canadians over 60 years to pay for it, we conducted an online survey to assess the knowledge, beliefs, and attitudes of older Canadians.

METHODS: The survey was piloted and assessed for content validity and test-retest reliability, and distributed electronically by the Canadian
Association of Retired Persons (CARP)/ 50Plus network to its members. RESULTS: 602 Canadians $\geq 60 \mathrm{y}$ from across Canada responded, of which $48 \%$ had shingles previously and $82 \%$ had a friend or family member suffer from shingles. Of those with shingles, $40 \%$ had postherpetic neuralgia. The vast majority (87\%) would accept the shingles vaccine if recommended by their physician and believed that the vaccine should be publicly funded $(87 \%)$. This was not significantly different if respondents previously had shingles, nor did it vary by age, education level or province of residence. Variables significantly associated with willingness to receive the vaccine were: previous incidence of chickenpox and willingness to receive a vaccine if indicated safe and recommended by a physician. Variables associated with belief that the government should pay for the vaccine were: willingness to receive a vaccine indicated as safe and recommended, and belief that the government should pay for all recommended vaccines. The majority $(68 \%)$ of respondents said they would receive shingles vaccine even if they had to pay for it themselves. Variables significantly associated with willingness to pay for the shingles vaccine included: belief that shingles can cause pain after the rash has disappeared, and willingness to be vaccinated against shingles if recommended by a physician. CONCLUSIONS: The majority of respondents either had shingles or knew someone who has, and the vast majority would be willing to accept shingles vaccine if recommended by their physician suggesting demand for this vaccine will be high. Although most respondents indicated that they would be willing to pay for the shingles vaccine, the majority felt that it should be publicly funded.

\section{SP19 \\ COMPARATIVE GENOMICS ANALYSIS OF CLOSTRIDIUM DIFFICILE: IMPROVING METHODS FOR DETECTION, EPIDEMIOLOGY, THERAPEUTICS AND UNDERSTANDING PATHOGENICITY}

M Oughton ${ }^{1 *}$, V Forgetta ${ }^{1}$, I Brukner ${ }^{2}$, A Villeneuve ${ }^{1}$, G Leveque $^{1}$, C Nagy ${ }^{1}$, J Dias ${ }^{1}$, V Loo ${ }^{3}$, V Magrini ${ }^{4}$, M Hickenbotham ${ }^{4}$, K Haub ${ }^{4}$, C Markovic $^{4}$, J Nelson ${ }^{4}$, E Mardis ${ }^{4}$, A Dascal ${ }^{2}$, K Dewar ${ }^{1}$

${ }^{1}$ McGill University and Genome Quebec Innovation Centre; ${ }^{2}$ SMBD-Jewish General Hospital; ${ }^{3}$ McGill University Health Centre, Montreal, QC; ${ }^{4}$ Genome Sequencing Centre, Washington

University, St. Louis, Missouri, United States

OBJECTIVE: Clostridium difficile has caused increasingly frequent and severe disease across North America and Europe over the last seven years. By comparative analysis of genomes from several C. difficile isolates, we plan to measure their genetic diversity. This comparison will be used to: (a) select targets for diagnosis and epidemiology; (b) identify polymorphisms potentially contributing to differential virulence; (c) choose candidate genes for development of active and passive immunization strategies. METHODS: C. difficile isolates were selected from clinical and reference collections, representing a range of strains, geographic sources, years of isolation, and disease severity. Genomic DNA was shotgun sequenced using the ultra-high-throughput Roche/454 GS FLX system, with finishing performed by PCR-directed gap closure. Genomic data was displayed on a local version of the UCSC Genome Browser, and annotated with gene predictions using Glimmer, with some manual editing.

RESULTS: Six of nine C. difficile isolates have been sequenced (as of the date of abstract submission). Genome sizes ranged between 4.04-4.35 Mb, containing approximately 3800 open reading frames (ORFs) at a coding density of $84 \%$. Read coverage was not uniform across all genomes, likely due to mobile genetic elements and extrachromosomal DNA. From approximately 2000 ORFs with conserved length between isolates, over 700 ORFs with $100 \%$ amino acid identity between all isolates were identified. Polymorphisms discriminating NAP1 from non-NAP1 isolates were present in over $400 \mathrm{ORFs}$, and a subset of 19 had at least six amino acid changes per ORF. Analysis of conventional and putative virulence factors (toxins, regulatory factors, antimicrobial resistance genes, surface and adhesion molecules) demonstrated polymorphisms between isolates.

CONCLUSIONS: ORFs conserved in all C. difficile isolates provide targets for nucleic acid-based species detection, while annotation of this set has identified some candidates suitable for vaccine development. ORFs 
with variation between NAP1 and non-NAP1 strains are suitable for nucleic acid-based assays that discriminate between strains, as well as providing a reduced set of ORFs that help explain differences in organism and disease phenotype.

\section{SP20}

THERE IS NO WAY THAT THIS IS INFECTIOUS, OR IS THERE?

T Papneja*, M Alawi, S Dixit, S Haider

McMaster University, Hamilton, ON

BACKGROUND: The differential diagnosis for neuromuscular disorders is broad and includes both non-infectious causes and infectious causes. Non-infectious causes of such disorders are more common and can result in both clinical and diagnostic dilemmas.

CASE PRESENTATION: A 50-year-old female developed dysphagia, blurred vision, and dysarthria. Within 3-4 days of the onset of her symptom her condition progressed rapidly to the point where she had complete ophthalmoplegia, quadriparesis and respiratory failure requiring intubation. None of her contacts were ill. Her past medical history was significant for Crohn's disease with multiple bowel resections. She was never on steroids. Physical examination revealed that her cognition was intact as she was able to minimally flicker her finger in respond to discussion of her illness. She had bilateral ptosis, bilateral dilated pupils unreactive to light, and flaccid quadriplegia except for minimal movement of her ankles and fingers. Her reflexes were intact despite the significant quadriparesis.

INVESTIGATIONS: Initial neuromuscular investigations narrowed the differential diagnosis to Eaton-Lambert Syndrome, Myasthenia Gravis versus Acute Motor Axonal Neuropathy (a motor variant of Gullian-Barre Syndrome). She was treated empirically with intravenous immunoglobulin (IVIG), steroids and pyridostigmine bromide for these diagnoses with no improvement in her condition. After thorough serological testing, lumbar puncture, and electrophysiological investigations that include high frequency stimulation between 20 and $50 \mathrm{~Hz}$, she was diagnosed with acute motor axonal neuropathy and assistance of the Infectious Disease team was no longer felt to be necessary. That is, until the lab called to say that the mouse died.

\section{SP21}

\section{UNUSUAL INFECTIOUS COMPLICATIONS CAUSED BY CLOSTRIDIUM PERFRINGENS}

\section{S Nayan*, C Lee, N Singhal}

\section{McMaster University, Hamilton, ON}

OBJECTIVE: To raise awareness about the potential role of $\mathrm{C}$. perfringens in severe, complicated infections and to report two cases in which C. perfingens was the unsuspected etiologic agent: a mycotic aneurysm and vertebral osteomyelitis.

\section{CASE REPORTS}

Case 1: An 80 year old male presented with increasing exertional shortness of breath and dysphagia. Lab investigations revealed a microcytic anemia and an elevated leukocyte count. Chest CT revealed an enlarging mycotic aneurysm in the right brachiocephalic artery as well as a second aneurysm in the subclavian artery with gas locules in the surrounding soft tissues. All other arteries were found to be normal. Blood cultures grew C. perfingens. The patient was deemed to be inoperable and was treated conservatively with antibiotics.

Case 2: A 72 year old female was admitted due to worsening back pain radiating to both legs. A month earlier a lumbar CT revealed vertebral compression fractures at L1 to L4, which were attributed to osteoporosis. These changes were not noted on a bone scan six months prior to her admission and on further probing she had had an episode of fever and back pain 5 months earlier. An MRI of her spine demonstrated a rim enhancing focus in the area of the L1 and L2 vertebral bodies with destruction of the vertebrae consistent with osteomyelitis and L1/L2 discitis. CT guided biopsy drained purulent material which grew C. perfingens. After four weeks of antibiotic therapy the patient underwent surgical debridement of the abscess.

CONCLUSION: Clostridium perfringens is most commonly involved in skin and soft tissue or gastrointestinal infections. We report here two cases where clostridial species are rarely implicated and C. perfringens is even less common. In both instances appropriate investigations were imperative to obtaining the correct diagnosis to guide therapy and prevent any further complications.

\section{SP22 \\ EVALUATION OF THE THREE DOSE SCHEDULE OF CONJUGATE PNEUMOCOCCAL VACCINE}

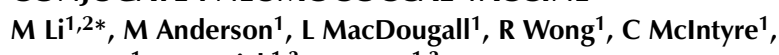
M Mclvor ${ }^{1}$, D Patrick $^{1,3}$, M Naus ${ }^{1,3}$

${ }^{1}$ British Columbia Centre for Disease Control; ${ }^{2}$ University of British Columbia, Master of Health Administration (MHA) Program;

${ }^{3}$ University of British Columbia, Department of Health Care and Epidemiology, Vancouver, BC

OBJECTIVES: Invasive pneumococcal disease (IPD), caused by the bacterium Streptococcus pneumoniae, is most common in the very young, the elderly and certain specific groups at high-risk. In 2003, a routine 4-dose infant immunization program of conjugated 7 -valent pneumoccocal vaccine was introduced (2, 4, 6 and 18 months). In 2007, we moved to a 3 dose schedule $(2,4,12$ months). In this study, we quantify the cost savings achieved, examine changes in immunization coverage rates and review vaccine effectiveness indicators over the first year of the schedule change. METHODS: Cost savings: The overall number of vaccine doses purchased and distributed in fiscal year 2008 will be compared with the data from fiscal years 2003-2007. Monthly distribution will be used to evaluate the lag in program uptake. Immunization coverage rates: Immunization coverage rates will be calculated using data from the immunization registry. IPD coverage rates following 3-dose implementation will be compared with 4dose rates for the previous period. Coverage pre- and post-schedule change will also be evaluated for other vaccines given at 12 months to ensure there has been no decrease in their uptake. Vaccine effectiveness: Data on IPD cases will be extracted from provincial reporting systems. Vaccine failure and program failure will be defined and differentiated among cases on 4-dose and 3-dose schedules. The age, geographic distribution and risk factor information of vaccine failure cases will be studied.

RESULTS: Preliminary data analysis shows that there has been good uptake of the three-dose vaccination schedule. In the 2006 birth cohort, following program implementation, $66 \%$ of children received two doses of pneumococcal vaccine at nine months of age. This is compared to a similar proportion, $67 \%$, receiving three doses prior to program implementation. Additionally, no vaccine failures have been reported and the target range on cost saving is $\$ 2$ million. The preliminary report will be available by March 2008 .

CONCLUSION: It is expected that the 3-dose vaccine schedule will save approximately $\$ 2$ million in fiscal year 2008 , that the immunization coverage rates have not declined prior to program change and that the 3 -dose schedule will not result in increased vaccine or programmatic failure.

\section{SP23}

MULTI-DRUG RESISTANT (MDR) SEROTYPE 19A STREPTOCOCCUS PNEUMONIAE (SPN) MENINGITIS IN A CHILD IN ONTARIO

W Ciccotelli ${ }^{1,5}$, A Opavsky ${ }^{1,4}$, P Cox $^{1,4}$, U Allen ${ }^{1,4}$, M Alqahtani ${ }^{1,4}$, N Rau ${ }^{3,4}$, D Pillai ${ }^{3,4}$, A McGeer ${ }^{3,4}$, DE Low ${ }^{2,3,4}$, SM Poutanen ${ }^{2,3,4}$ ${ }^{1}$ The Hospital for Sick Children; ${ }^{2}$ University Health Network; ${ }^{3}$ Mount Sinai Hospital; ${ }^{4}$ University of Toronto, Toronto; ${ }^{5}$ McMaster University, Hamilton, ON

OBJECTIVES: The prevalence of invasive pneumococcal disease (IPD) caused by SPN in children and adults has decreased following the introduction of Prevnar ${ }^{\circledR}$. However, a concomitant rise in the incidence of disease due to the MDR serotype 19A SPN non-vaccine strain has occurred. A recent severe case of meningitis and sepsis due to MDR 19A SPN in a fully vaccinated healthy child in Ontario is described.

METHODS: A clinical chart review was completed. Susceptibility testing used broth microdilution (BMD), Etest (AB Biodisk, Sweden), or disk diffusion (DD). Susceptibility results were interpreted following CLSI 
standards. The SPN isolate was serotyped using latex pool antisera and pneumococcal antisera (Statens Serum Institut). Confirmation of SPN serotyping was confirmed by the National Centre for Streptococcus in Edmonton.

RESULTS: A 14-m old child presented to hospital following 2 days of fever and vomiting. IV cefuroxime was initiated. The following day, lethargy, leukopenia and thrombocytopenia were noted. CSF exam revealed bacterial meningitis (WBC $63 \times 10^{6} / \mathrm{L}$, glucose $0.1 \mathrm{mM} / \mathrm{L}$, protein $3.6 \mathrm{~g} / \mathrm{L}$, gram-positive cocci in pairs and chains). Treatment was changed to IV vancomycin and ceftriaxone, and the patient was transferred to a tertiary care ICU. Shock and multi-organ failure developed. CSF and blood cultures were positive for 19A SPN. MICs, by BMD unless otherwise specified, revealed resistance to penicillin $(4 \mathrm{mg} / \mathrm{L})$, amoxicillin $(8 \mathrm{mg} / \mathrm{L})$, erythromycin $(>32 \mathrm{mg} / \mathrm{L})$, clindamycin $(>16 \mathrm{mg} / \mathrm{L})$, trimethoprim-sulfamethoxazole $(8 / 152 \mathrm{mg} / \mathrm{L})$, tetracycline $(8 \mathrm{mg} / \mathrm{L})$, cefprozil $(16 \mathrm{mg} / \mathrm{L})$, cefuroxime $(16 \mathrm{mg} / \mathrm{L})$, ceftriaxone $(2 \mathrm{mg} / \mathrm{L})$, and meropenem $(1 \mathrm{mg} / \mathrm{L}$, Etest). The strain was susceptible to vancomycin $(\leq 0.5 \mathrm{mg} / \mathrm{L})$, levofloxacin $(\leq 0.25 \mathrm{mg} / \mathrm{L})$, telithromycin $(0.5 \mathrm{mg} / \mathrm{L})$, and rifampin $(36 \mathrm{~mm}, \mathrm{DD})$. Ceftriaxone was changed to levofloxacin, and vancomycin was continued with clinical response. Rifampin was avoided due to liver impairment.

CONCLUSIONS: The emergence of invasive MDR 19A SPN disease in Canada reinforces the importance of empiric therapy with vancomycin and cefotaxime (or ceftriaxone) for children or adults with suspected or confirmed bacterial meningitis and for critically ill patients with suspected or confirmed nonmeningeal invasive pneumococcal disease. Canada-wide laboratory-based surveillance for IPD due to MDR 19A should be initiated.

\section{SP24}

FIRST RECOVERY OF AN ENVIRONMENTAL BACTERIUM, AURANTIMONAS ALTAMIRENSIS FROM CLINICAL MATERIAL

M Luong1*, V Leung', G Al-Rahani' ${ }^{2}$, D Lauzon ${ }^{3}$, S Bekal ${ }^{4}$, D Vinh'1, B Ng ${ }^{5}$ A Reimer ${ }^{5}, \mathrm{~T}$ Burdz $^{5}$, D Wiebe ${ }^{5}, \mathrm{~K}_{\text {Bernard }}{ }^{5}$

${ }^{1}$ McGill University Health Centre, Montreal, QC; ${ }^{2}$ Children's \& Women's Health Centre of British Columbia, Vancouver, BC; ${ }^{3}$ Hopital St-Jean du Haut-Richelieu, St. Jean; ${ }^{4}$ Laboratoire de Santé Publique du Québec, INSPQ, Sainte-Anne-de-Bellevue, QC; ${ }^{5}$ National Microbiology Laboratory, Winnipeg, MB

OBJECTIVE: Three isolates of an environmental bacterium, Aurantimonas altamirensis found to be associated with two clinical cases, are presented. To the best of our knowledge, this is the first description of this agent being recovered from clinical materials.

METHODS: We reviewed specimens that were identified as Aurantimonas spp from the Laboratoire de Santé Publique du Québec and the National Microbiology Laboratory (NML). Microbiologists and treating physicians involved in each index case were contacted to obtain clinical information by chart review. Bacteria identified as Aurantimonas altamirensis were characterized using standard phenotypic, chemotaxonomic and genetic methods. Antimicrobial susceptibility testing was done based on CLSI guidelines.

RESULTS: A gram-negative bacillus was isolated from specimens from three different hospitals: contact lens fluid, corneal scraping (patient 1) and sputum secretion (patient 2, with cystic fibrosis [CF]). Routine identification kit with API 20E strip identified the organism as Ochrobactrum anthropi with 95\% agreement; API NE strip was unable to provide an acceptable identification. Using $16 \mathrm{~S}$ rRNA gene sequencing and phylogenetic analysis, the specimens were unambiguously identified as Aurantimonas altamirensis. Extensive biochemical analysis and fatty acid content analysis performed on all three isolates were in agreement with those described for this species.

CONCLUSIONS: We describe here the first report of cases of Aurantimonas altamirensis isolated from human clinical specimens. Although the pathogenic role of this organism is unknown, particularly in the CF patient, we demonstrated here that this bacterium could be recovered from clinical specimens. The use of $16 \mathrm{~S}$ rRNA gene sequencing provided a more accurate taxonomic designation than traditional biochemical or kit based methods alone.

\section{SP25}

MYCOBACTERIUM HAEMOPHILUM EPIDIDYMAL ABSCESS IN A RENAL TRANSPLANT PATIENT

M Keller ${ }^{1}$, A Mak ${ }^{1 *}$, L Thibert ${ }^{2}$, P René ${ }^{1}$, M Klein ${ }^{3}$

${ }^{1}$ Department of Microbiology, McGill University Health Centre,

Montreal, QC; ${ }^{2}$ Laboratoire de santé publique du Québec, SainteAnne-de-Bellevue, QC; ${ }^{3}$ Department of Medicine, Division of Infectious Diseases, McGill University Health Centre, Montreal, QC

BACKGROUND: Mycobacterium haemophilum is an established cause of cutaneous lesions in immunocompromised hosts and has also been reported as a rare cause of osteomyelitis, arthritis, and cervical lymphadenitis in immunocompetent children.

CASE REPORT: A 57 year-old man with diabetes and end stage renal disease requiring renal transplantation presented with a two day history of scrotal swelling and pain. He appeared well and was afebrile. On examination, the right scrotum was enlarged and tender, but there was no overlying cellulitis, skin lesions, or palpable nodes. Chest x-ray revealed multiple calcified granulomata that were unchanged from previous films. The patient was brought to the operating room and the tunica vaginalis was opened to reveal a necrotic epididymus. A right orchiectomy was performed and the pus was sent to our microbiology laboratory for bacterial and mycobacterial culture. The acid-fast stain of the abscess revealed 4+ bacilli but PCR was negative for the $M$ tuberculosis complex. The specimen was inoculated onto two sets of MGIT culture tubes and LowensteinJensen culture broths, both supplemented with hemin. One set was incubated at $37^{\circ} \mathrm{C}$ and the other at $30^{\circ} \mathrm{C}$. Once growth was detected in the MGIT culture tube, the samples were sent to the provincial laboratory where 16S DNA sequencing demonstrated $100 \%$ sequence identity with partial sequence of type strain M haemophilum ATCC 29548.

CONCLUSION: $M$ haemophilum is a slow growing organism that requires a low incubation temperature $\left(30^{\circ} \mathrm{C}\right)$ and hemin for growth. This is the first described case of $\mathrm{M}$ haemophilum epididymal abscess in a renal transplantation patient. Our case highlights the need to consider this diagnosis when setting up mycobacterial cultures of specimens from immunocompromised patients.

\section{SP26}

EVALUATION OF QUIKI-CULT URINE SCREENING SYSTEM A Belhaj ${ }^{*}$, C Lee ${ }^{1,2}$, C Main ${ }^{1,2}$, D Yamamura ${ }^{1,2}$, L Wilcox ${ }^{2}$, J Paget $^{2}$, K Power $^{2}$

${ }^{1}$ McMaster University; ${ }^{2}$ Hamilton Regional Laboratory Medicine Program, Hamilton, ON

BACKGROUND: Urine cultures are the most common specimen in Microbiology laboratories. However, many yield no growth, insignificant colony counts, or mixed growth. We compared the accuracy of two screening methods: Bayer Multistix 8 SG (Bayer Inc, Toronto, Canada) and the QuikiCult System, Ime Inc, (Maryland, U.S.A).

METHODS: Consecutive urines were run in parallel on the Multistix 8 SG (Multi) and the QuikiCult (QuikiC) as per the manufacturer's instructions. The plunger of the QuikiC device is pushed, breaking a seal which allows entry of approximately $4.8 \mathrm{ml}$ of urine which reconstitutes a freezedried medium. The device is incubated for 30 minutes and then read in the QuikiC reader. The data transferred to a program and interpreted. A baseline reading is compared to the reading after incubation for 3 hours aerobically at $35^{\circ} \mathrm{C}$. Interpretation is based on a colour change (TTC changes from colourless to red upon bacterial growth) and change in biomass as detected by an infra-red reading. Urines were cultured using a $0.001 \mathrm{ml}$ loop onto blood agar and MacConkey's agar which were incubated aerobically at $35^{\circ} \mathrm{C}$ for $18-24$ hours. Interpretation and investigation of cultures were based on standard microbiology procedures.

RESULTS: 602 urines were examined. 84 urines showed the presence of 1 or 2 pathogens, or 1 pathogen mixed a urogenital contaminant, at a colony counts of $>10 \times 10^{6} \mathrm{CFU} / \mathrm{L}$. The QuikiC detected $66(79 \%)$ and the Multistix 64 (77\%). At the colony count $>100 \times 10^{6} \mathrm{CFU} / \mathrm{L}$ of a urine pathogen in pure culture, The QuikiC detected $60(90 \%)$ and the Multistix 57 (85\%). Of the 518 urines that showed no growth, insignifi- 
cant growth, or mixed growth of 3 or more organisms, 18 and 139 were positive by the QuikiC and Multistix respectively. The overall sensitivity and specificity were 79(95\% CI 68-86) and 97 (94-98) for QuikiC and 76 (65-84) and 73 (69-76) for the Multistix.

CONCLUSION: The QuikiC, a novel urine screening method, is as sensitive and more specific the Multistix. Using QuikiC "no growth" or "mixed growth" urines are reported the day received. There is a potential for saving due to reduction in the number of cultures that would be investigated.

\section{SP27}

CHANGING THE CULTURE OF AN INSTITUTION: SHARPS INJURIES AND THE PERCEPTION AND ATTITUDES TOWARD THEM AT A CANADIAN CENTRE

J Nadarajah*, C Main

McMaster University, Hamilton, ON

OBJECTIVE: Sharps injuries are a serious problem in the healthcare setting, as they present the possibility of transmitting blood borne infections. However, there seems to be a culture of reluctance to report injuries in our hospitals. The aim of this study was to examine the rates, attitudes and knowledge regarding sharps injuries amongst resident physicians at McMaster University.

METHODS: A comprehensive 52-piece questionnaire was distributed to residents of all specialties. Data was obtained anonymously.

RESULTS: 110 questionnaires were returned. Respondents were all residents of medical and surgical specialties, ranging from one to five years of experience. $58 \%$ of respondents had sustained at least one sharps injury in the past four years culminating in a total of 102 injuries. $47 \%$ of these injuries were reported. $41 \%$ of residents did not report any of their injuries. $71 \%$ of all injuries occurred within 1 year of experience as a resident. $40 \%$ of injuries had at least one high risk feature. $64 \%$ of injuries were reported within four hours of the injury. Of the residents who did not report an injury, $69.2 \%$ of them claimed it was because they did not perceive it to be a high risk injury, but $5 \%$ of them knew the serological status of the patient. $32.3 \%$ of respondents had received formal training on sharps injuries at some time in their career. Fluid splashes, scratches, bites and spicules of bone or teeth were not considered to be an exposure or reportable by $63 \%$ of respondents. $84.5 \%$ of residents were not aware of the official institutional protocol on needlestick and body fluid exposure, and of those who were aware, $5 \%$ had seen the protocol on display in hospitals. Goggles and face shields were used when indicated an average of $72 \%$ of the time, while gloves were used $93 \%$ of the time when handling contaminated materials. Contaminated needles were recapped an average of $39 \%$ of the time. $44 \%$ of residents did not believe their workplace was fully committed to having supports in place for the proper education, prevention and efficient post-exposure protocols in dealing with sharps injuries. CONCLUSION: This study identifies a high rate of needlestick injuries in residents and highlights a high rate of underreporting. The most common reason for not reporting an injury was a perception of low risk. The attitudes and perceptions towards sharps injuries, combined with a minimal amount of knowledge and training is likely contributing to the high rates of injury and low rates of reporting. In answer to this study, the institution is currently working on protocols to enhance awareness of sharps injuries and change current attitudes and knowledge in this regard.

\section{SP28}

TERMINAL GRAM STAINS AND SUBCULTURES OF STERILE FLUIDS SUBMITTED IN BLOOD CULTURE VIALS: IS THERE A NEED?

J Carson ${ }^{1 *}$, U Chandran ${ }^{1,2}$

${ }^{1}$ University of Alberta; ${ }^{2}$ Dynacare Kasper Medical Laboratories, Edmonton, $A B$

OBJECTIVE: The use of blood culture media and automated blood culture systems for the recovery of bacteria from normally sterile body fluids has improved recovery by $20-40 \%$ when compared to conventional culture methods. Few studies address the need for the terminal subculture of negative vials. In order to assess the utility of this practice, we evaluated the laboratory outcomes of all sterile body fluids submitted in BACTEC/F media with respect to their terminal smears and subcultures.

METHODS: We retrospectively examined all sterile body fluids received over a one year period. The terminal Gram stain and subculture results were reviewed for specimens submitted in BACTEC vials (BACTEC Plus aerobic, anaerobic and/or pediatric $\mathrm{F}$ medium). All vials were incubated on the BACTEC 9240 automated blood culture instrument. If negative at the end of a five day incubation period, a terminal Gram stain and subculture (chocolate and CDC anaerobic agar) were performed. If growth occurred, the organism(s) was identified by standard methods.

RESULTS: In the one year period, $284(6.3 \%)$ of 4491 sterile body fluid specimens were cultured in a total of 500 BACTEC vials; $216(76.1 \%)$ in aerobic and anaerobic vials, $42(4.8 \%)$ in aerobic vials, $14(4.9 \%)$ in anaerobic and $12(4.2 \%)$ in pediatric vials only. Thirty-seven $(15.1 \%)$ specimens from 63 vials yielded positive cultures within five days. Terminal gram stains and subcultures were performed on the remaining 247 specimens (437 vials), of which six $(2.4 \%)$ had positive gram stains and subcultures (anaerobic [4], pediatric [1], aerobic [1]). The organisms recovered were Corynebacterium CDC group G (2), Propionibacterium acnes (2), anaerobic gram positive cocci (1), coagulase negative staphylococci (1) and Staphylococcus aureus (1). The specimen with S. aureus had a 36h delay in transit and no other corresponding cultures. All other specimens with S. aureus (10) were positive within five days.

CONCLUSION: Terminal smears and subculture of sterile fluids from negative BACTEC vials creates significant extra work in the laboratory with very low yield. Transport times greater than $24 \mathrm{~h}$ may require terminal gram stain and culture to ensure a true positive culture is not missed.

\section{SP29}

DETECTION OF MRSA BY THE BD GENOHM MRSA ASSAY (BMA) FROM FLOCKED SWABS TRANSPORTED IN LIQUID AMIES (LA)

D Goldfarb $^{3 *}$, P Jessamine ${ }^{1,2}$, A Bonneau ${ }^{1}$, K Ramotar ${ }^{1,2}$, $M$ Desjardins ${ }^{1,3}$

${ }^{1}$ The Ottawa Hospital, Ottawa, ON; ${ }^{2}$ The Ottawa Hospital Research Institute, Ottawa, ON; ${ }^{3}$ Children's Hospital of Eastern Ontario, Ottawa, ON

WITHDRAWN

\section{SP30}

\section{COMMUNITY ACQUIRED METHICILLIN RESISTANT} STAPHYLOCOCCUS AUREUS IN LONDON, ONTARIO: A 30 MONTH RETROSPECTIVE STUDY

Z Chagla $^{1 *}$, M Salvadori ${ }^{2}$, Z Hussain ${ }^{2}$, Y Chagla ${ }^{2}$, M John $^{2}$

${ }^{1}$ Queen's University, Kingston; ${ }^{2}$ London Health Sciences Centre, London, ON

OBJECTIVE: Methicillin Resistant Staphylococcus aureus (MRSA) is a known cause of morbidity and mortality in the hospitalized population. Community acquired MRSA (CA-MRSA), in otherwise healthy individuals is of increasing concern to the Canadian population. There is a greater need for characterization of CA-MRSA infections in Canada, in order to improve recognition and treatment. The objective of this study is to examine CA-MRSA cases in London, Ontario, and assess the epidemiology, clinical characteristics, and laboratory features of this infection.

METHODS: Cases were defined using the Pulse Field Gel Electrophoresis patterns seen in most Canadian CA-MRSA infections. Information on patient demographics, risk factors, clinical characteristics, treatment, outcomes, and antibiograms were obtained retrospectively from charts over a 30 month interval. Paediatric and adult data were statistically compared. Analysis of isolates from the Emergency Department (ED) over a 6 month interval were compared to methicillin-sensitive Staphylococcus aureus isolates over the same interval, to estimate the proportion of CA-MRSA infections in the outpatient setting.

RESULTS: 44 patients were identified with CA-MRSA infection, of which $27 \%$ (12) were paediatric, and $83 \%$ (32) were adult. A 6 month subgroup analysis showed that $6 \%$ of Staphylococcus aureus infections in the ED were CA-MRSA. Risk factors included medical comorbidities, 
antibiotic use in the past year, and exposure to CA-MRSA positive individuals. $98 \%$ of cases had at least one risk factor. Although most patients presented with skin/soft tissue infections (81\%), invasive infections were seen in $11 \%$. After culture and sensitivity results, $75 \%$ of patients were treated with a CA-MRSA sensitive antibiotic, although paediatric cases were treated with sensitive drugs significantly more than adults $(\mathrm{p}<0.05)$. $52 \%$ of patients were admitted or were already in hospital at diagnosis. Cases were typically CMRSA10 (89\%), with resistance mainly to erythromycin (89\%)

CONCLUSION: The emergence of CA-MRSA cases has been seen in this institution, and presented as a cause of morbidity in hospitalized patients, those with comorbidities, and in otherwise healthy individuals. Health care professionals need to include CA-MRSA in the differential diagnosis of skin/soft tissue infections, especially if a risk factor is present.

\section{SP31}

RISK FACTORS FOR COMMUNITY AND HOSPITALASSOCIATED MRSA INFECTIONS IN PATIENTS PRESENTING TO EMERGENCY DEPARTMENTS WITH STAPHYLOCOCCAL SOFT TISSUE INFECTIONS IN A MAJOR CANADIAN CITY

H Adam ${ }^{1}$, V Allen ${ }^{1}$, B Borgundvaag ${ }^{2}$, A Currie ${ }^{3}$, A McGeer ${ }^{2,1}$, A Simor ${ }^{4,1}$, S Richardson ${ }^{1,5}$, L Louie ${ }^{4}$, B Willey ${ }^{2}, K_{\text {Katz }}{ }^{1,3}$, Emergent Working Group ${ }^{1}$

${ }^{1}$ University of Toronto; ${ }^{2}$ Mount Sinai Hospital, Toronto; ${ }^{3}$ North York General Hospital, North York; ${ }^{4}$ Sunnybrook Health Sciences Centre; ${ }^{5}$ The Hospital for Sick Children, Toronto, ON

OBJECTIVES: Methicillin resistant Staphylococcus aureus (MRSA) in Canada has been predominantly associated with hospital acquisition; however, clusters of community associated MRSA (CA-MRSA) have been described. The primary objective of this study was to determine the current prevalence of CA-MRSA in patients with purulent skin and soft tissue infections (SSTIs) presenting to emergency departments (EDs) in a major Canadian city. Secondary objectives included the ascertainment of risk factors, analysis of clonality, and antimicrobial susceptibility patterns. METHODS: This prospective descriptive study was designed to compare patients who presented to six EDs with purulent SSTI caused by MRSA to those caused by methicillin susceptible S. aureus (MSSA) from March $1^{\text {st }}$ to June $30^{\text {th }}, 2007$. Patients presenting to the participating EDs with purulent SSTI were notified of the study. Treating physicians were asked to take swabs for microbiologic analysis and treat according to their usual practice. All patients with confirmed S. aureus were contacted for a voluntary telephone-based interview. Susceptibility testing was performed. All MRSA isolates were sub-typed by pulsed field gel electrophoresis, and PCR for the presence of the pvl toxin gene and staphylococcal chromosomal cassette mec typing was performed.

RESULTS: 298 patients were identified with a SSTI due to S. aureus. 54 (18\%) of these were due to MRSA. Based on PFGE patterns, $29(54 \%)$ of the 54 MRSA isolates were typical community strains (CMRSA-10), were SCCmec type IV and had the pvl gene. Interviews were completed for 161/ 298 (54\%) of eligible patients; individuals with CA-MRSA infections had fewer known exposures to MRSA ( $p=0.02$ ); fewer healthcare-related risk factors $(\mathrm{p}=0.04)$; and less antibiotic use in the previous 3 months $(\mathrm{p}<0.01)$. There was a trend towards younger ages in those with CAMRSA ( $p=0.09$ ). In contrast to the multi-drug resistant patterns of HAMRSA, all of the CA-MRSA isolates were susceptible to clindamycin, trimethoprim-sufamethoxazole, tigecycline, and tetracycline and the majority were susceptible to daptomycin.

DISCUSSION: The prevalence of MRSA in purulent SSTIs is $18 \%$ (54/298). 54\% (29/54) of the MRSA isolates were CMRSA-10. Follow-up prevalence studies are required to track the evolution of CA-MRSA.

\section{SP32}

MRSA BLOOD STREAM INFECTION: EPIDEMIOLOGIC AND CLINICAL CHARACTERISTICS IN ONE INSTITUTION OVER A SEVEN YEAR PERIOD

\section{J Minion*, L Saxinger, L Chui, J Fuller, G Taylor}

University of Alberta, Edmonton, AB

OBJECTIVE: Hospitals frequently track the occurrence of MRSA, however rarely distinguish between colonization and infection. Colonization rates will be sensitive to the intensity of surveillance and since colonization has no clinical consequence, these rates will misrepresent the burden of disease due to MRSA. Tracking MRSA blood stream infections (BSI) is a more reliable marker of MRSA occurrence as it is not dependent on surveillance method, and more closely reflects burden of disease. The objective of this study was to characterize the cases of MRSA bacteremia at our institution during a 7 year period.

METHODS: A prospectively collected, single hospital Infection Control database was searched for all MRSA BSI for the years 2000 through 2006. 36 patients were identified. Reviews of patient charts, electronic health records, and the hospital laboratory information system were performed. Molecular characterization by PFGE and PVL gene detection was performed on available isolates.

RESULTS: There were 36 episodes of MRSA BSI, ranging from 0-17/years. The mean patient age was 59 and $69 \%$ were male. ICU (22\%) and General Internal Medicine (17\%) were the most common admitting services. The case rate was $2.2 / 100,000$ days over the 7 years, ranging from 0 in 2002 to 6.6 in 2006 with an overall increasing trend. Pneumonia (31\%) and primary BSI (29\%) were the most common sources of infection. $54 \%$ of patients were still hospitalized at 1 month and there was $29 \%$ overall hospital mortality. Resistance to non-beta-lactam antibiotics was common, including 92\% ciprofloxacin, 89\% clindamycin, and 53\% TMP/SMX resistance. Vancomycin MICs ranged from 0.5 to $4 \mu \mathrm{g} / \mathrm{mL}$ (median $\mathrm{MIC}=2$ ), with a trend over time towards increasing vancomycin MICs. Adequate empiric therapy was given in $71 \%$ of cases. Prior MRSA colonization was known in $69 \%$ of patients, of which $92 \%$ were given adequate empiric therapy.

CONCLUSION: While still rare, MRSA BSI rates seem to have increased over the last 7 years. Known colonization identified by screening can be helpful in guiding empiric therapy vancomycin MICs may be increasing.

\section{SP33 \\ DETECTION OF FUSIDIC ACID RESISTANCE IN \\ METHICILLIN-RESISTANT STAPHYLOCOCCUS AUREUS (MRSA): VERIFICATION OF E-TEST AND DISC DIFFUSION METHODOLOGIES}

P Gnanasuntharam ${ }^{1,2}$, BM Willey ${ }^{1,3}$, V Porter ${ }^{1}$, S Malek ${ }^{1}$, S PongPorter $^{1}$, Y Rzayev ${ }^{1}$, DE Low ${ }^{1,2,3,4}$, SM Poutanen ${ }^{1,2,3}$

${ }^{1}$ Mount Sinai Hospital; ${ }^{2}$ University of Toronto; ${ }^{3}$ University Health Network; ${ }^{4}$ Central Public Health Laboratory, Toronto, ON

BACKGROUND: While fusidic acid (FA) is common in topical ointments, it may be used orally for staphylococcal osteomyelitis and for eradication of doxycycline/co-trimoxazole-resistant (R) MRSA carriage. In MRSA, FA acts on fusA, the bacterial elongation factor-G (EF-G) gene, interrupting protein synthesis. EF-G mutations correlate with FA MIC $12->256 \mathrm{mg} / \mathrm{L}$, while acquisition of fusB leads to MIC $2-8 \mathrm{mg} / \mathrm{L}$. As MRSA with $\mathrm{MIC}>1 \mathrm{mg} / \mathrm{L}$ are common in Canada, laboratories should perform susceptibilities when FA is used. Using CLSI broth microdilution, this study evaluated the ability of E-test and disc diffusion (DD) to detect FA-R MRSA.

METHODS: In total, 270 MRSA from 2000-05 were selected to include 95 (35.2\%) FA-R, 1 (0.3\%) FA-intermediate (FA-I) and 174 (64.4\%) FA-S, based on MIC breakpoints of Skov et al. 2001, where 1mg/L=FA-I. E-test and DD were done as per CLSI using 10ug FA discs on Mueller-Hinton agar. E-test MIC and DD zone diameters were independently read by 5 technologists blinded to each others results and to the published breakpoints of $>20 \mathrm{~mm}$ for FA-S and $<19 \mathrm{~mm}$ for FA-R. The total 1,350 reads for each method were plotted against the MIC of each strain to determine the 
distribution frequencies.

RESULTS: E-test agreed 100\% with MIC for all 1,350 reads (95\% CI 99.7-100\%) from the 270 MRSA (95\% CI 99.8-100\%). By DD, 286 $(21.2 \%)$ reads were $<19 \mathrm{~mm}, 89(6.6 \%)$ were $19-20 \mathrm{~mm}$ and 975 $(72.2 \%)$ were $>20 \mathrm{~mm}$ (range $21-41 \mathrm{~mm}$ ). Comparison of DD reads to CLSI MIC found 100 very major errors (VME) $(21 \%$, 95\% CI $17.6-25 \%)$ that included $76(5.6 \%)$ at MIC $2 \mathrm{mg} / \mathrm{L}(21-26 \mathrm{~mm}), 23$ $(1.7 \%)$ at $4 \mathrm{mg} / \mathrm{L}(21-25 \mathrm{~mm})$ and 1 at $8 \mathrm{mg} / \mathrm{L}(21 \mathrm{~mm}) ; 94$ minor errors (ME), that included 5 FA-S instead of FA-I (100\%; 95\% CI 51-100\%) and 89 FA-I instead of FA-R (18.7\%, 95\% CI 15.5-22.5\%); there were no major errors (MJE) $(0 \%, 95 \%$ CI $0-0.53 \%)$. Use of an alternative DD breakpoint of $<26 \mathrm{~mm}$ for FA-R resolved 99/100 VME $(0.2 \%, 95 \%$ CI $0.01-1.31 \%)$ and all ME but resulted in $12 \mathrm{MJE}(1.4 \%, 95 \% \mathrm{CI}$ $0.76-2.42 \%$ ).

CONCLUSIONS: This study verified the FA E-test against CLSI MIC using published criteria. However, if 10 ug FA discs are to be used, the alternative DD breakpoint of $<26 \mathrm{~mm}$ for FA-R should be considered as this resulted in acceptable combined error rate of $<3 \%$.

\section{SP34 \\ MOLECULAR EPIDEMIOLOGY AND ANTIBIOTIC \\ SUSCEPTIBILITIES OF METHICILLIN-RESISTANT STAPHYLOCOCCUS AUREUS (MRSA) IN EMERGENCY ROOM (ER) PATIENTS OVER 6 YEARS}

A Rostas*, BM Willey, E Cudek, M Moussa, DE Low

Mount Sinai Hospital, Toronto, ON

BACKGROUND: MRSA in the Toronto community has increased in recent years. To show this change in epidemiology, and to determine its impact and the implications for laboratory susceptibility testing and reporting, this study analyzed yearly trends and characteristics of MRSA identified from patients treated at a downtown Toronto ER.

METHODS: Data from the laboratory information system was used to identify ER patients with S. aureus from Jan 2000-Dec 2006. One MRSA per patient was typed by SmaI PFGE. Inducible clindamycin resistance (clindR) was detected using the CLSI double disc test (DDT) in erythromycin-resistant (eryR) MRSA that were clind-susceptible (S) by Vitek. RESULTS: Overall, $26.8 \%$ of the 749 ER patients growing S. aureus had MRSA; by 2006, 46\% of S. aureus in ER patients were MRSA. A summary of the data is tabled below:

S. aureus MRSA CMRSA PF types (\% of MRSA):

\begin{tabular}{lccccccccc} 
Year & (Total no.) & No. & Ery \%R & Clind \%R & $\mathbf{1}$ & $\mathbf{2}$ & $\mathbf{5}$ & $\mathbf{1 0}$ & Other \\
\hline 2000 & 82 & 20 & 95 & 95 & 70 & 20 & 5 & 0 & 5 \\
2001 & 70 & 9 & 78 & 78 & 89 & 0 & 11 & 0 & 0 \\
2002 & 90 & 13 & 92 & 92 & 31 & 38 & 0 & 0 & 31 \\
2003 & 96 & 15 & 100 & 93 & 40 & 27 & 20 & 0 & 13 \\
2004 & 98 & 19 & 89 & 74 & 10.5 & 63 & 10.5 & 10.5 & 5.5 \\
2005 & 146 & 49 & 92 & 69 & 2 & 61 & 0 & 23 & 14 \\
2006 & 167 & 76 & 99 & 52 & 7 & 39 & 0 & 47 & 7 \\
Overall & 749 & 201 & 94.5 & 69.6 & 19.9 & 42.3 & 3.5 & 24.4 & 9.9 \\
\hline
\end{tabular}

Until 2003, eryR predicted clindR (ermA/C), but by 2006, 35/75 (47\%) of eryR MRSA were clindS by DDT $(m s r A)$. PFGE showed the once predominant CMRSA-1 in decline, the established presence of hospitalassociated CMRSA-2, and the emergence of community-acquired CMRSA-10 (USA300) to become the predominant MRSA in ER patients by 2006.

CONCLUSIONS: The significant increase in patients with eryR/clindS CMRSA-10 $(\mathrm{P}=0.0018)$ highlights the importance of implementing routine DDT of eryR MRSA from the ER.
SP35

DETECTION OF LOW- AND HIGH-LEVEL MUPIROCIN RESISTANCE IN METHICILLIN-RESISTANT STAPHYLOCOCCUS AUREUS (MRSA): EVALUATION OF MUPIROCIN SCREEN AGARS

V Porter ${ }^{1 *}$, SM Poutanen ${ }^{1,2,3}$, N Kreiswirth ${ }^{1}$, E Cudek ${ }^{1,2}$, N Nelson ${ }^{1,2}$, S Malek ${ }^{1,2}$, A McGeer ${ }^{1,2,3}$, DE Low ${ }^{1,2,3,4}$, BM Willey ${ }^{1,2}$ ${ }^{1}$ Mount Sinai Hospital; ${ }^{2}$ University Health Network; ${ }^{3}$ University of Toronto; ${ }^{4}$ Public Health Laboratory, Toronto, ON

BACKGROUND: The incidence of mupirocin resistance (MupR) in Toronto MRSA has been increasing due to two epidemic clones. One clone, indistinguishable from CMRSA-2, has mutation-mediated lowlevel (LL) MupR, and the other, CMRSA-9, carries the mupA gene that encodes high-level (HL) MupR. In areas where topical Mup ointment is used for MRSA eradication therapy, routine testing of each patient's initial MRSA isolate is recommended. Four agars, each with a different Mup concentration, were compared with the aim of developing a simple, reliable and cost effective method to detect MupR in MRSA.

METHODS: In total, 369 MRSA were selected to include Mup MIC of $0.038-1024 \mathrm{mg} / \mathrm{L}$ (as per CLSI broth microdilution). Using proposed Mup breakpoints $(4 \mathrm{mg} / \mathrm{L}=\mathrm{R})$, the strains comprised 209 Mup-S $(0.038-2 \mathrm{mg} / \mathrm{L})$, 142 LL-MupR (4-128mg/L) and 18 HL-MupR (256->1024mg/L). As per CLSI screen agar methodology, bacterial suspensions equivalent to 0.5 McFarland STD were spotted to Mueller Hinton agars containing Mup at $4,6,8$ and $10 \mathrm{mg} / \mathrm{L}$, respectively. Incubation was at $35^{\circ} \mathrm{C}$ in ambient air. Spot plates were examined using transmitted light at $18 \mathrm{~h}$ and $24 \mathrm{~h}$. Any growth was considered to indicate resistance.

RESULTS: No major errors ( $0 \%, 95 \%$ CI 0-2.8\%) occurred on any screen agar regardless of Mup concentration or length of incubation. No very major errors (VME) $(0 \%$; $95 \% \mathrm{CI} 0-2.2 \%)$ occurred on the Mup4mg/L agar, but 2 VME were found on Mup6mg/L (1.2\%; 95\% CI 0.04-3.65\%) and 3 VME were found on Mup8mg/L and Mup10mg/L agars $(1.9 \%$; $95 \%$ CI $0.29-4.3 \%)$. For the Mup4mg/L agar, the sensitivity, specificity, positive and negative predictive values were $100 \%, 100 \%, 100 \%$ and $100 \%$ for $18 \mathrm{~h}$ and $24 \mathrm{~h}$ reads. For the $6 \mathrm{mg} / \mathrm{L}$ agar, these values were $98.8 \%$, $100 \%, 100 \%$ and $99.1 \%$ at both reads, while on the $8 \mathrm{mg} / \mathrm{L}$ and $10 \mathrm{mg} / \mathrm{L}$ Mup agars these values were $98.1 \%, 100 \%, 100 \%$ and $98.6 \%$ at both reads, respectively.

CONCLUSIONS: The 4mg/L Mueller Hinton Mup screen agar was highly sensitive and specific and effectively detected all LL- and HLMupR in the MRSA with no errors. The screen agar method was easy to perform and may be readily incorporated into existing laboratory workflow. To distinguish between LL- and HL-MupR in the MRSA, PCR testing for the mupA gene or MIC testing via E-test or CLSI broth microdilution would be needed.

\section{SP36 \\ DETECTION OF VEROTOXIN-PRODUCING ESCHERICHIA COLI BY MOLECULAR APPROACH}

C Cheung $^{1 *}$, T Chiu ${ }^{1}$, B Lee ${ }^{1}$, R McDonald ${ }^{2}$, N Antonishyn ${ }^{2}$, L Chui ${ }^{1}$ ${ }^{1}$ Provincial Laboratory for Public Health, Edmonton, AB;

${ }^{2}$ Saskatchewan Disease Control Laboratory, Regina, SK

INTRODUCTION: Hemolytic uremic syndrome (HUS) is well-known as a severe complication associated with diarrheal illness caused by E. coli O157:H7, a verotoxin-producing Escherichia coli (VTEC). HUS has also been reported after non-O157 VTEC infections and some studies have found that up to $35 \%$ of VTEC infections were from non-O157 E. coli. $\mathrm{CHROMagar}^{\mathrm{TM}}$ and MacConkey plates are currently used in the majority of diagnostic microbiology laboratories to detect E. coli O157, but nonO157 VTEC will be missed by this approach. Our study is to detect VTEC in paediatric stool samples using a real-time PCR assay, and to isolate and further characterize the $E$. coli strains in the positive samples.

METHODS: Stool samples from 544 paediatric patients submitted for viral investigation from 2001-2002 were included. An aliquot of each sample was inoculated in broth media and incubated at $37^{\circ} \mathrm{C}$ overnight. DNA was extracted from the broth by rapid lysis technique and tested for VTEC by real-time PCR. Positive samples were plated on selective media 
and six E. coli colonies from each plate were randomly picked for VTEC testing by the real-time PCR and verotoxin-positive strains were serotyped.

RESULTS: Of the 544 stool samples, 9 tested positive for verotoxin gene-1 $(n=5)$, verotoxin gene-2 $(n=3)$ and both $(n=1)$. Only 2 of these samples cultured positive for E. coli and both contained non-O157 E. coli. One isolate tested positive for verotoxin by PCR and was typed as E. coli O26. The E. coli from the second sample tested negative for verotoxin by PCR.

CONCLUSIONS \& DISCUSSION: We found the presence of nonO157 VTEC in stool samples from paediatric patients with suspected viral gastroenteritis. Our real-time PCR was able to detect non-O157 VTEC, which would have been missed by the conventional culture method which targets E. coli O157. While only one VTEC was recovered by culture, it is possible that the other 8 verotoxin-positive samples contained viable but non-culturable VTEC that were not isolated by the conventional culture plate method, which further supports the superior sensitivity of using realtime PCR to detect VTEC. Another advantage of the real-time PCR is the rapid turn-around time for reporting results to physicians, allowing earlier monitoring for post-infection complications.

\section{SP37 \\ MOLECULAR DETECTION OF GIARDIA LAMBLIA IN SURFACE WATER: COMPARISON TO GOLD-STANDARD METHOD}

N Prystajecky ${ }^{1 *}$, P Huck ${ }^{2}$, J Isaac-Renton ${ }^{1,2}$

${ }^{1}$ University of British Columbia, Vancouver, BC; ${ }^{2}$ University of Waterloo, Waterloo, ON; ${ }^{3}$ British Columbia Centre for Disease Control, Vancouver, BC

INTRODUCTION: In British Columbia (BC) there have been 29 confirmed outbreaks of waterborne gastrointestinal illness since 1980, nearly half of which were attributed to Giardia lamblia. The gold standard method for detection of Giardia in water is Method 1623, an immunological microscopic method developed by the United States Environmental Protection Agency. This method, however, cannot determine human health risk and as such, efforts to develop an equivalent molecular method have been made. This study evaluates molecular methods in comparison to the gold standard method.

METHODS: Surface water was collected biweekly in the Township of Langley, BC, over a 2-year period and processed according to Method 1623. In brief, up to $50 \mathrm{~L}$ of water was collected by filtration. The resulting eluate was processed by immunomagnetic separation (IMS) and enumerated microscopically by an indirect immunofluorescent assay (IFA). Following microscopy, cysts were recovered by slide-scraping and genomic DNA was isolated by freeze-thaw and proteinase $\mathrm{K}$ digestion. Amplification and sequencing at two loci (beta-giardin gene and 18s rRNA gene) was carried out in duplicate on positive and negative slides.

RESULTS: By IFA, Giardia was detected in $844 \%$ of surface water samples, at concentrations ranging from 0 to 160 cysts per $100 \mathrm{~L}$ of water. $65 \%$ (beta-giardin gene) and $77 \%$ (18s rRNA gene) of samples positive by IFA were also positive by PCR. Few samples $(<10 \%)$ that were negative by IFA were positive by PCR. Sequence analyses demonstrated that the majority of samples (85\%) contained human infective, zoonotic isolates (Assemblages A and B).

DISCUSSION: Molecular methods are frequently investigated to supplement or replace gold standard laboratory methods. The results reveal that while Methods 1623 provides limited health risk data by providing detection to only the genus level, it demonstrated a greater detection limit and repeatability and thus may be more suitable for routine testing. Supplementation with molecular analyses may be best suited for ecological and epidemiological studies.
SP38

TRENDS IN INCIDENCE OF INVASIVE PNEUMOCOCCAL DISEASE (IPD) FOLLOWING INTRODUCTION OF THE UNIVERSAL INFANT IMMUNIZATION PROGRAM, BRITISH COLUMBIA (2002-2006)

N Shahidi ${ }^{1,3 *}$, J Dhaliwal ${ }^{1}$, G Tyrrell ${ }^{2}$, L Hoang ${ }^{1,3}$, D Patrick ${ }^{1,3}$

${ }^{1}$ British Columbia Centre of Disease Control, Vancouver, BC; ${ }^{2}$ National Centre of Streptococcus, Edmonton, $\mathrm{AB} ;{ }^{3}$ University of British Columbia, Vancouver, BC

BACKGROUND: Streptococcus pneumoniae is a leading cause of pneumonia, bacteremia, sepsis and meningitis worldwide. We analyzed reporting of invasive pneumococcal disease (IPD) associated with the infant pneumococcal conjugate vaccine (PCV-7) program and assessed evidence for serotype replacement.

METHODS: Data on IPD cases from 2002-2006 were extracted from the integrated Public Health Information System (iPHIS) and BCCDC laboratory database. Incidence rates were calculated by obtaining population estimates for the denominators from P.E.O.P.L.E 30. Trends over time were explored with the chi-square test for trend.

RESULTS: There was a 70\% reduction in rate of IPD, from 2002-2006 among children $<5$ years of age. Although the overall rate of IPD in BC was constant from 2002-2005, it increased by $40 \%$ from 2005 to 2006. This occurred largely among those aged 20-64 who saw a significant increase from 4.6 to 11.0 cases $/ 100,000$ population $\left(\chi^{2}=87\right.$; $\left.p<0.00001\right)$. This was driven by a significant increase in IPD in that age group caused by strains not covered by PCV 7 and related to an outbreak of serotype 5 IPD. There was no significant increase in non-PCV 7 IPD in children, adolescents or the elderly.

CONCLUSION: A decrease in the incidence rate for $<5$ age group is associated with the PCV-7 program. An increase in IPD during 2006 related to a clonal outbreak in adults rather than to serotype replacement in the immunized population.

\section{SP39}

\section{THE IMPACT OF ROUTINE IMMUNIZATION USING} MENINGOCOCCAL C CONJUGATE VACCINE ON INVASIVE MENINGOCOCCAL DISEASE IN BRITISH COLUMBIA AND ITS IMPLICATIONS FOR FUTURE IMMUNIZATION

\section{STRATEGIES}

T Siu 1,2*, W Tang1 ${ }^{1}$ M Dawar ${ }^{2,3}$, D Patrick ${ }^{1,2}$

${ }^{1}$ British Columbia Centre for Disease Control; ${ }^{2}$ University of British Columbia, Vancouver, BC; ${ }^{3}$ Canadian Field Epidemiology Program, Public Health Agency of Canada, Ottawa, ON

OBJECTIVE: In Canada, Neisseria meningitidis is the leading cause of bacterial meningitis in children 5-19 years of age. In 2003, British Columbia (BC) initiated a routine childhood protein-polysaccharide conjugate $\mathrm{C}$ (MCC) immunization program. The objectives of our study were to 1) examine trends in serogroup- $\mathrm{C}$ incidence associated with the MCC program, 2) assess for evidence of capsule switching and serogroup replacement, and 3) examine whether findings support modification of the current MCC program to include quadrivalent protein-polysaccharide conjugate vaccine (MCV-4).

METHODS: Information on invasive meningococcal disease (IMD) cases reported since 1998 were extracted from an enhanced surveillance database. Data management and calculations were performed using Microsoft ${ }^{\circledR}$ Office Excel 2003. Time trends were analyzed using chi-square test for linear trend with StatCalc (EpiInfo 6).

RESULTS: While annual rates of serogroup-specific and total IMD in the $\mathrm{BC}$ population have remained unchanged ( $\mathrm{p}>0.05$ for linear trends) since 2003, serogroup-C pediatric (children $<18$ years) IMD annual rates have declined from 0.65 cases $/ 100,000$ persons in 2003 to zero case in 2006 with a statistically significant downward trend $(\mathrm{p}=0.0048)$. Median age of serogroup-C IMD increased from 16 years (2003) to 42 years (2006). In contrast to serogroup-C disease, pediatric IMD from non- $\mathrm{C}$ serogroups has remained unchanged for this time period ( $p>0.05$ for linear trends).

CONCLUSION: We show a decreasing trend of pediatric serogroup-C IMD and an increase in the median age of serogroup-C IMD cases since 
2003. A reasonable explanation is the protection conferred by the childhood MCC Immunization program. The proportion of serogroup-Y IMD has increased in recent years. However, incidence of $\mathrm{Y}$ or other non- $\mathrm{C}$ vaccine preventable IMD has not increased in BC. This is important when considering possible inclusion of MCV-4 in childhood immunization programs.

\section{SP40 \\ PREVALENCE OF CHRONIC HEPATITIS B/HEPATITIS C CO-INFECTION AND VACCINE PREVENTABLE ACUTE HEPATITIS IN INDIVIDUALS WITH CHRONIC HEPATITIS IN BRITISH COLUMBIA}

S Padhi ${ }^{1 *}$, JA Buxton ${ }^{2}$, M Krajden ${ }^{2}, \mathrm{~A} \mathrm{Yu}^{2}$

${ }^{1}$ University of Ottawa, Ottawa; ${ }^{2}$ British Columbia Centre for Disease Control, Vancouver, BC

OBJECTIVES: Infection with more than one hepatitis virus may increase liver disease progression. However, little is known about population prevalence of co-infection. Hepatitis in BC is reported into the integrated Public Health Information System (iPHIS); hepatitis B Virus (HBV) is reported as acute, chronic or undetermined. The objectives of this study are: 1) estimate the prevalence of individuals co-infected with chronic $\mathrm{HBV}$ and hepatitis $\mathrm{C}$ virus (HCV) in BC; 2) study the sequence of virus detection; 3) estimate the prevalence of vaccine preventable acute hepatitis after chronic infection identified i.e. acute HBV in persons previously diagnosed with HCV, and acute hepatitis A (HAV) in persons with chronic HBV \&/or HCV; 4) describe demographic characteristics (sex, age group, residence) of co-infected individuals and evaluate trends.

METHODS: Individuals with HAV, HBV and HCV infections reported into iPHIS (1991-October 2007) were identified. Undetermined and chronic HBV were considered chronic. Those with more than one infection were identified and all personal identifiers were removed. Date of coinfection was the date $2^{\text {nd }}$ virus was identified; diagnosis was considered simultaneous if the viruses were reported within 14-days.

RESULTS: A total of 35,189 individuals with chronic/undetermined HBV and 58,080 individuals with HCV were identified. Of these 1815 were co-infected with $\mathrm{HCV} / \mathrm{HBV} ; 72 \%$ were male; $53 \%$ were aged $30-44$ years; males $>$ females in all age groups except $10-14$ and $15-19$ years; $50 \%$ were reported simultaneously and in 26\% HBV reported first. 196 acute HBV infection post-HCV infection were reported; HAV was reported in 176 individuals post diagnosis of chronic HBV or HCV infections. Rates of HBV/HCV co-infection have declined since 2000.

CONCLUSION: $>1800$ persons in BC are HBV/HCV co-infected. Despite provincial vaccination guidelines, individuals with $\mathrm{HCV}$ or chronic HBV infection were subsequently identified infected with vaccine preventable acute hepatitis infection. This study highlights the need for adequate follow-up and immunization of persons identified with chronic $\mathrm{HBV}$ and $\mathrm{HCV}$.

\section{SP41}

VALIDITY OF ELECTRONIC SURVEILLANCE SYSTEMS: A SYSTEMATIC REVIEW

\section{J Leal*, K Laupland}

University of Calgary, Calgary, AB

OBJECTIVE: Although surveillance is widely recognized as a major aspect of infection prevention and control, conventional methods are labor intensive and costly. There has been an increased interest in development of electronic surveillance systems that utilize existing data in large microbiology and administrative databases. Our objective was to systematically review published studies comparing the utility of electronic with conventional surveillance systems.

METHODS: Publications comparing electronic and conventional surveillance systems were searched using MEDLINE(1980-2007) and bibliographic review. Inclusion articles had to compare electronic systems against a conventional "gold standard" method. Sensitivity (Sn) and specificity $(\mathrm{Sp})$ of electronic compared to conventional surveillance was reported. Articles that only evaluated the timelines or completeness of electronic reporting were excluded.
RESULTS: Twenty-five studies were included of which 21 (84\%) focused on nosocomial infections and $4(16 \%)$ community-acquired infections. Five articles evaluated the utility of laboratory databases for case identification (Sn 79-93\%; Sp 91->99\%). Two articles used laboratory-based algorithms to detect infection outbreaks yielding variable utility measures (Sn 43\% \& 57\%; Sp 67\% \& 83\%). Two studies used stepwise logistic regression using computerized surveillance systems to determine characteristics that predicted nosocomial infections and found Sn $81 \%$ and $63 \%$ and Sp $98 \%$ and $87 \%$, respectively. Three articles evaluated the accuracy of using only laboratory data $(\mathrm{Sn} 72-86 \%)$ or its combination with one (Sn 79-90\%) and two (Sn 49-94\%) other infection indicators. Six studies combined ICD-9-CM discharge and admission diagnoses with laboratory and antibiotic use databases to identify infections and found Sn $8.4-90 \%$ and Sp 95->99\%. Health maintenance organizations' databases used in five studies found Sn $53-100 \%$ and Sp $75-99 \%$ for the identification of post-discharge and community-acquired infections. One article utilized electronic radiology reports and found that they could detect nosocomial pneumonia in neonates with Sn $71 \%$ and $\mathrm{Sp}>99 \%$. An electronic syndromic surveillance system detected bioterrorism-related respiratory illness in a single article with Sn 68\% and Sp 98\%. Electronic surveillance reduced surveillance time by up to $61 \%$.

CONCLUSION: The current small body of literature shows that electronic surveillance has moderate to high utility but is more efficient than conventional methods. Future studies are needed to refine electronic algorithms further, particularly with community onset infections.

\section{SP42 \\ PREVALENCE AND CHARACTERISTICS OF QUINOLONE- RESISTANT NEISSERIA GONORRHOEAE ISOLATES IN ONTARIO}

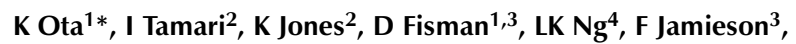
A Diprima $^{3}$, S Richardson ${ }^{1}$

${ }^{1}$ The Hospital for Sick Children; ${ }^{2}$ Hassle Free Mens Clinic;

${ }^{3}$ Ontario Ministry of Health and Long-Term Care, Toronto, ON; ${ }^{4}$ National Microbiology Laboratory, Public Health Agency of Canada, Winnipeg, MB

OBJECTIVE: After declining for a number of years, Neisseria gonorrhoeae (NG) infections have recently made a resurgence in Canada. National surveillance has shown that the prevalence of antimicrobial resistance in NG is also increasing with at least $20 \%$ of all NG isolates demonstrating resistance to at least one antibiotic. Recently, quinolone-resistant NG (QRNG) has emerged in Canada, increasing from 2.1\% in 2001 to $15.7 \%$ in 2005. American studies have associated QRNG with men who have sex with men (MSM), HIV infection, older age, and travel to Asia. This study aimed to establish the prevalence of QRNG in Ontario and to identify risk factors for QRNG infection in a Canadian setting.

METHODS: This retrospective cohort study used records from the Central Public Health Laboratory, Toronto, Ontario, to identify all culture-positive NG isolates from 2006. Limited demographic data was extracted from all ciprofloxacin-resistant QRNG $(\mathrm{MIC} \geq 1.0 \mu \mathrm{g} / \mathrm{mL})$ and from a random sample of ciprofloxacin-sensitive (QSNG) isolates at a 1:1 (QRNG to QSNG) ratio. The controls were selected randomly with even spread over the 12 months.

RESULTS: Of the 2499 NG isolated in 2006, 700 (28.0\%) were QRNG. 570 QSNG controls were selected. QRNG had proportionately more men than QSNG (OR 2.22, 95\% CI 1.62-3.05). The proportion of MSM was similar in both groups (OR $0.87,95 \%$ CI $0.69-1.10$ ). Male gender remained a statistically significant predictor of QRNG after controlling for MSM status $(p<0.001)$. QRNG had a higher proportion of patients over 30 years of age (OR 2.95, 95\% CI 2.34-3.71). QRNG isolation increased on average $8 \%$ per month ( $95 \%$ CI $6-10 \%)$, but significant summertime seasonality was also seen $(\mathrm{p}=0.001)$. Poisson regression showed a statistically significant surge of QRNG isolates in August, 2006 after controlling for seasonal oscillation and the background increase in QRNG $(\mathrm{p}<0.001)$. CONCLUSIONS: Twenty-eight percent of NG isolated in Ontario in 2006 were QRNG. Heterosexual men appeared to be contributing significantly to the QRNG rate. MSM status did not appear to be an independent risk factor for QRNG acquisition. The 2006 Canadian Guidelines on 
Sexually Transmitted Infections recommends that quinolones be avoided where QRNG rates are higher than 3-5\%. Health care practitioners should be aware of the widespread prevalence of QRNG in Ontario and treat cases accordingly.

\section{SP43 \\ DETECTION OF CLASS 1, 2 AND 3 INTEGRONS IN BACTERIA USING A REAL-TIME TRIPLEX PCR AMPLIFICATION AND EVALUATION OF ITS METHODOLOGY}

JH Xiong ${ }^{*}$, M Blais ${ }^{2}$, N Messier ${ }^{1}$, PH Roy ${ }^{1,2}$

${ }^{1}$ Centre de Recherche en Infectiologie, Centre Hospitalier de I'Université Laval; ${ }^{2}$ Department de Biochimie, I'Université Laval, Ste-Foy, QC

OBJECTIVE: Class 1, 2 and 3 Integrons are important capture and expression systems for antibiotic resistance genes. A real-time triplex PCR was designed and used for detecting class 1, 2 and 3 integrons from bacterial lysates.

METHODS: Bacteria: 127 clinical isolates of bacteria mainly resistant to at least two kinds of antibiotics including 60 Pseudomonas spp., 24 E. coli, 17 Klebsiella spp., 19 other Enterobacteriaceae and 7 Staphylococcus aureus were used as tested samples. A panel of 35 class 1 integron positive control strains, 30 known class 1 integron negative strains (confirmed by hybridisation with probes from 5'CS and 3'CS and sequencing) were also included in the test. E. coli (JM109) strains (pLQ860, pLQ420 and pLQ422) carrying class 1, 2 and 3 integrase genes individually were used specifically in each of real-time triplex PCR runs.

Method: Three sets of primers targeted to integrase 1, 2 and 3 genes were designed and used in the real time triplex PCR using Taq DNA polymerase (BIOTOOLs, Madrid, Spain), Rotor Gene 3000 and bacterial lysates. The fluorescent SYBR Green I was incorporated in the reaction so that the melting curves were obtained at the rates of $0.2^{\circ} \mathrm{C} / \mathrm{s}$ for differentiation of the tested PCR products against positive and negative controls.

RESULTS: Class 1 integron was predominant in clinical isolates and control strains. More rarely, classes 2 and 3 can be seen. 34/60 (57\%) of Pseudomonas spp., 16/24 (67\%) of E. coli, 11/17(65\%) of Klebsiella spp., 10/19(53\%) of other Enterobacteriaceae and 1/7(14\%) of Staphylococcus aureus isolates were found positive for class 1 integrons. Four clinical isolates (one E. coli and 3 other Enterobacteriaceae) were positive for class 2 integron while no class 3 integron was found in the clinical isolates. The specificity and sensitivity of the test were $97 \%$ and $100 \%$, respectively. Specific class 1, 2 and 3 integron controls gave constant positive results during each PCR run.

CONCLUSION: The real-time triplex PCR is a fast, simple, economical and reliable tool for use in epidemiological surveys of antibiotic resistancecarrying classes 1, 2 and 3 integrons.

\section{SP44}

CHARACTERIZATION OF CEFOXITIN-RESISTANT ESCHERICHIA COLI FROM WATER SOURCES IN CANADA L Mataseje ${ }^{1,3 *}$, N Neumann ${ }^{2}$, P Baudry ${ }^{1}$, G Zhanel ${ }^{1}$, M Louie ${ }^{2}$, M Mulvey ${ }^{1,3}$, AROWater Study Group ${ }^{2}$

${ }^{1}$ University of Manitoba, Winnipeg, MB; ${ }^{2}$ Alberta Provincial Public Health Laboratory, Calgary, AB; ${ }^{3}$ National Microbiology Laboratory, Winnipeg, MB

OBJECTIVE: E. coli resistant to cephalosporins has become a major concern in communities and hospitals. In this report we describe the molecular epidemiology and mechanisms of cefoxitin-resistance (FOX-R) from E. coli identified from drinking water across Canada.

METHODS: 3747 E. coli isolates from beach and well water were collected from Alberta, Quebec and Ontario spanning 2004-2006. Antimicrobial susceptibilities were determined using microdilution broth method following CLSI guidelines. All isolates with an MIC of $>16 \mathrm{mg} / \mathrm{L}$ for FOX were screened for the ampC genes DHA, FOX, ENT, CIT in a multiplex PCR followed by sequence analysis. Chromosomal ampC promoter regions were sequenced in plasmidic AmpC negative strains. All FOX-R isolates were typed into phylogenetic groups using PFGE with
XbaI. Plasmidic AmpC isolates were characterized by RFLP patterns digested with BgIII and a multiplex PCR for replicon typing.

RESULTS: A total of 125/3052 (4.1\%) E. coli water isolates displayed FOX-R. 77/125 (61.6\%) exhibited multidrug resistance (MDR) defined as resistance to $\geq 2$ different antibiotic classes. 94/125 (75.2\%) of isolates contained the bla $\mathrm{CMY}_{2}$ gene. $29 / 125(23.2 \%)$ isolates were negative for all ampC genes tested, though sequencing of the ampC promoter region showed mutations from the wild-type previously shown to hyperproduce ampC. Of the CMY-2 isolates, 88/94 (94\%) showed unique PFGE patterns. Plasmid analysis of CMY-2 transformants revealed 20/94 (21.3\%) MDR. Replicon typing revealed $b l a_{\mathrm{CMY}-2}$ plasmids predominately repI1 $(52.1 \%)$ followed by repA/C (14\%) and repK/B (14\%). 11/94 (11.7\%) of isolates contained $>1$ replicon type. RFLP patterns of plasmid transformant DNA revealed 3 clusters with $>80 \%$ homology corresponding to prevalent replicon types.

CONCLUSION: $4.1 \%$ of E. coli obtained from well water sources displayed FOX-R with the majority being bla $\mathrm{CMY}_{\mathrm{C}}$. Incompatibility group repI1 is the most prevalent of the CMY-2 plasmids. Comparison of human clinical cases of FOX-R E. coli will provide insight into the potential water-borne sources of infection.

\section{SP45}

MOLECULAR CHARACTERIZATION OF ESCHERICHIA COLI RESISTANT TO TRIMETHOPRIM-SULFAMETHOXAZOLE ISOLATED FROM URINARY TRACT INFECTIONS IN NORTHERN SASKATCHEWAN COMMUNITIES

NC Persaud 1,2*, H Nguyen 1, GR Golding ${ }^{2}$, PN Levett ${ }^{3}$, RR McDonald ${ }^{3}$, J Irvine ${ }^{3}$, MR Mulvey $^{3}$ and Northern Antimicrobial Resistance Partnership

${ }^{1}$ University of Manitoba; ${ }^{2}$ National Microbiology Laboratory, Winnipeg, MB; ${ }^{3}$ Saskatchewan Disease Control Laboratory, Regina, SK

OBJECTIVE: Antimicrobial resistance in urinary tract infections (UTIs) is of concern as it limits treatment options. Approximately $80 \%$ of UTI cases are caused by uropathogenic E. coli (UPEC). The objective of this study was to characterize trimethoprim-sulfamethoxazole (TMP-SXT) resistant E. coli identified from three northern Saskatchewan communities.

METHODS: Isolates were collected through the Northern Antibiotic Resistance Partnership (NARP) over a 6 month period from July 2006-December 2006. Susceptibilities to 28 antibiotics were determined using microdilution broth assays following CLSI guidelines. Pulsed-field gel electrophoresis (PFGE) using XbaI was used to type the isolates. Class I integrons among isolates were identified by PCR and DNA sequencing. RESULTS: A total of 342 UPEC isolates were collected (Site 1=211, Site $2=104$, Site $3=27$ ). Most of the UPEC isolates were from female patients (females $=90 \%$, males $=4 \%$, no data $=6 \%$ ). The highest percentage of UTI patients were between the ages of 20-24 years old (13\%). TMP-SXT resistance was observed in 111 isolates (Site $1=33 \%$, Site $2=26 \%$, Site 3 $=56 \%)$. From the isolates that were TMP-SXT resistant, $15 \%$ were resistant to only TMP-SXT, 35\% were resistant to one other antibiotic class, $39 \%$ were resistant to 2 classes, and $11 \%$ were resistant to $\geq 3$ classes. PFGE data showed that there were no large outbreaks of TMP-SXT resistant UPEC within or between the sites. Class 1 integrons were detected in 96 $(86 \%)$ of the TMP-SXT resistant isolates. Eleven different cassette arrangements were identified within the integrons which contained $d f r$, aadA, or a combination of the two cassettes. Trimethoprim resistance cassettes identified were $d f_{r} A 1, d f r A 7, d f r A 12, d f r A 17$, and $d f r V$. Streptomycin/spectinomycin resistant cassettes identified were aadA1, $\operatorname{aadA2}$, and aadA5.

CONCLUSION: The high rate of resistance to TMP-SXT observed among the sites, suggests that a possible re-evaluation of first line therapies for UTI treatment should be considered. The lack of strain clonality and the large number of resistance cassettes observed suggests resistance in the community is diverse and unlikely to have a common origin. 


\section{SP46}

HAS MANDATORY REPORTING OF HIV CHANGED THE PROBABILITY OF NEWLY DIAGNOSED PEOPLE BEING EARLY IN THEIR ILLNESS?

D Taylor ${ }^{1,5 *}$, M Krajden ${ }^{1,5}$, D Cook ${ }^{1}$, J Kim ${ }^{3}$, E Wong ${ }^{2}$, M Tyndall ${ }^{4,5}$, G Ogilvie $^{1,5}$, M Rekart ${ }^{1,5}$, D Patrick ${ }^{1,5}$

${ }^{1} \mathrm{BC}$ Centre for Disease Control, Vancouver, BC; ${ }^{2}$ Public Health Agency of Canada; ${ }^{3}$ National HIV Reference Lab, PHAC, Ottawa, $\mathrm{ON} ;{ }^{4} \mathrm{BC}$ Centre for Excellence in HIV/AIDS; ${ }^{5}$ University of British Columbia, Vancouver, BC

OBJECTIVE: On 1 May 2003, HIV was added to the list of reportable diseases in British Columbia, Canada, leading to enhanced surveillance and contact tracing. Sensitive/less-sensitive (S/LS) EIA testing has been used to monitor trends in recent seroconversions (i.e. less than 6 months) among newly identified cases. The primary objective of this study was to examine the association between HIV Reporting practice and testing for HIV within 6 months of becoming infected as determined by a S/LS EIA testing method.

METHODS: Banked HIV positive samples (confirmed by Western Blot), collected between 1 Jan 2000-30 Apr 2003 (pre-reporting group) and 1 May 2003-23 Aug 2006 (post-reporting group) were re-tested using the bioMérieux Vironostika HIV-1-S/LS tests. Samples were classified by the S/LS EIA as recent infection (infected for $<170$ days) or established infection (>170 days). The proportion of individuals who were recently infected in the pre-reporting group was compared to the post-reporting group using a Pearson's Chi-Square. The hypothesis was that due to enhanced follow-up and partner notification there would be $>5 \%$ increment in the proportion of recent infections among people testing newly positive in the post-reporting group compared to the pre-reporting group. RESULTS: Serum was available for 1111 newly positive HIV cases in the pre-reporting group and 470 in the post-reporting group. This consisted of 2149 males (1086 pre; 1063 post) and 569 females ( 275 pre; 294 post). In the pre-reporting group 311 (28\%; CI: $25.36 \%, 30.73 \%$ ) of cases were recent infections compared to 136 (29\%; CI: $24.87 \%, 33.27 \%)$ in the postreporting group $(\mathrm{p}=0.703$ ). It is important to note that the amount of serum available for this comparison did not provide enough power to detect a $5 \%$ increment in the proportion of recent infections between study groups.

CONCLUSIONS: The bioMérieux Vironostika HIV-1-S/LS test has proven to be an effective tool to objectively evaluate a public health intervention. While this test has not been accepted as a diagnostic or surveillance tool, the bias inherent in the test is equal between pre and post intervention groups. This underpowered study was unable to detect a difference in the proportion of individuals testing early after becoming infected with HIV between the pre and post reporting groups.

\section{SP47}

NOVEL MUTATION IN MYCOBACTERIUM TUBERCULOSIS CONFERRING RIFAMPIN MONORESISTANCE IN A CLUSTER OF HIV POSITIVE PATIENTS

\section{S Malhotra ${ }^{1 *}$, V Cook ${ }^{1,2}$, J Wolfe ${ }^{3}$, P Tang ${ }^{1,2}$, K Elwood ${ }^{1,2}$, M Sharma $^{3,4}$}

${ }^{1}$ University of British Columbia; ${ }^{2}$ British Columbia Centre for Disease Control, Vancouver, BC; ${ }^{3}$ National Microbiology Laboratory; ${ }^{4}$ University of Manitoba, Winnipeg, MB

OBJECTIVES: Rifampin is a key component of standard short-term firstline therapy against M. tuberculosis (MTB). Monoresistance to rifampin, previously a rare phenomenon is now being reported at increasing rates worldwide. Molecular studies reveal over $95 \%$ of mutations conferring rifampin monoresistance occur in an 81 base pair region, encoding the beta subunit of RNA polymerase $(r p o B)$. We report a novel mutation in the $r p o B$ region leading to high level rifampin resistance in a cluster of HIV positive patients.

METHODS: Patients were identified using susceptibility results from the British Columbia Centre for Disease Control, Vancouver, Canada. Isolates from all patients with rifampin monoresistant MTB infection from 2004 to 2006 were sent to the National Reference Centre for Mycobacteriology,
Winnipeg, Canada for susceptibility confirmation, sequencing of $r p o B$ and genotyping using Mycobacterial Interspersed Repetitive Unit - Variable Number Tandem Repeat testing (MIRU-VNTR).

RESULTS: Three patients were found to have a previously undocumented 3 base pair insertion at codon 525 in the rpoB region. The index case initially had fully susceptible tuberculosis. Disease relapse occurred seven months later with an MTB isolate showing acquired rifampin monoresistance. MTB isolates from two subsequent patients showed primary rifampin monoresistance with identical strain typing to the index case.

CONCLUSION: Patients with rifampin monoresistant tuberculosis tend to have poorer outcomes than those with fully susceptible strains. Risk factors for the development of rifampin monoresistance include co-morbid HIV infection and previously treated tuberculosis. HIV infection has been associated with malabsorption of antituberculous medications leading to subtherapeutic levels. These factors may have played a role in the development of this novel mutation.

\section{SP48}

\section{ANTIMICROBIAL RESISTANCE IN GONORRHEA: THE INFLUENCE OF EPIDEMIOLOGIC AND LABORATORY SURVEILLANCE DATA ON PROVINCIAL TREATMENT GUIDELINES IN ALBERTA, 2001 TO 2007}

\section{Boyington*, AE Singh, S Plitt, K Sutherland, M Lovgren, P Tilley,} G Tyrrell University of Alberta, Edmonton, AB

OBJECTIVE: To examine trends and associated demographic factors of antimicrobial resistance in gonorrhea and the impact on provincial treatment guidelines in Alberta.

METHODS: Demographics and risk factors from surveillance data collected by Alberta Health \& Wellness STD Services were linked to antimicrobial resistance data from the Provincial Laboratory for Public Health for all cases of ciprofloxacin resistant gonorrhea (CRG) between January 1, 2001 and June 30, 2007.

RESULTS: The number of gonorrhea cases in Alberta increased from 801 in 2001 to 2149 in 2006 and 1,046 in the first 6 months of 2007. In this same time period, the proportion of cases processed by culture declined from $335(42.2 \%)$ in 2001 to $672(31.3 \%)$ in 2006. The number and proportion of CRG by year was $3(1.4 \%)$ in 2001, $11(2.7 \%)$ in 2002, 21 $(5.5 \%)$ in $2003,15(3.3 \%)$ in $2004,30(5.9 \%)$ in $2005,70(10.3 \%)$ in 2006 and $67(21.8 \%)$ in the first 6 months of 2007. Of a total of 217 individuals between 2001 and 2007 with CRG, $90 \%$ were male with a median age of 29 (age range 16 to 61 ). In response to the rising proportion of CRG, enhanced surveillance was instituted for all CRG in 2004. Of the 158 CRG cases with enhanced surveillance from 2005-2007, 142 (90\%) were male and of these $70 \%$ were men who have sex with men (MSM) and an additional 10\% had reported sexual contact outside of Alberta. Among the 16 female CRG cases, $31 \%$ had reported sexual contact outside of Alberta or a sexual partner who had sexual contact outside of Alberta. Ciprofloxacin was the first line recommended treatment agent for the treatment of gonorrhea in Alberta since 1991; in November 2005, the treatment guidelines were changed to recommend cefixime as the preferred agent among MSM and in individuals reporting sexual contact either outside Alberta or with non-Albertans. In May 2007, provincial treatment guidelines were changed from ciprofloxacin to cefixime due to the observation of locally acquired cases of CRG among heterosexual persons.

CONCLUSIONS: CRG increased in Alberta between 2001 and 2007. Close monitoring of epidemiologic risk factors allowed a sequential change in treatment guidelines from empiric ciprofloxacin to cefixime. This study highlights the importance of ongoing monitoring of antimicrobial resistance to ensure appropriate treatment of gonorrhea in the future. 
SP49

COST EFFECTIVENESS OF A PUBLICLY FUNDED HEPATITIS B VACCINATION PROGRAM FOR BLOOD DONORS IN BRITISH COLUMBIA

S Waters ${ }^{1,2 *}$, J Buxton ${ }^{1,2}$, M Bigham ${ }^{3,4}$

${ }^{1}$ Health Care and Epidemiology, University of British Columbia;

${ }^{2} \mathrm{BC}$ Centre for Disease Control; ${ }^{3}$ Canadian Blood Services, Vancouver; ${ }^{4}$ Fraser Health Authority, BC

OBJECTIVE: To assess the cost effectiveness and benefits of providing a publicly funded hepatitis B virus (HBV) vaccination program in British Columbia (BC) to blood donors with no history of prior vaccination to help increase the safety of the blood supply.

METHODS: This analysis was taken from a health care payer perspective and had a deterministic approach using point estimates for both costs and effectiveness. The proportion of vaccine provided by physicians versus public health was estimated by Medical Health Officers and Public Health Nurses in the various regional Health Authorities. Public health delivery was assumed to be by unscheduled, non-mass immunization clinics. Fixed costs, such as space and utilities, were not included in this analysis. Variable costs consisted of direct and indirect costs. Direct costs included managing local vaccine and supplies, time and salaries of nursing and clerical staff to support immunization delivery within public health and Medical Services Plan billing payments to physicians for injections. Indirect costs included support to physicians to deliver immunizations outside of public health. A sensitivity analysis considered HBV vaccination history of blood donors and a range of vaccine uptake.

RESULTS: Approximately 3660 vaccines would be provided by private physicians at $\$ 17.63$ per dose for a three dose cost of $\$ 198,000$. Approximately 40,200 vaccines would be provided by public health at $\$ 22.00$ per dose for a three dose cost of $\$ 2,705,000$. Total costs of the program at $80 \%$ uptake of the vaccine would be approximately $\$ 2,323,000$. Considering variation in prior HBV vaccination history of blood donors from $10 \%$ to $30 \%$ gave a range in program cost from $\$ 2,037,000$ to $\$ 2,431,000$. Costs of the following year of the program accounting for immunization of new donors only would be approximately $\$ 382,000$. The program would potentially prevent about one transfusion-transmitted $\mathrm{HBV}$ infection in BC every two years.

CONCLUSION: A publicly funded HBV vaccination program for blood donors has low cost-effectiveness for increasing the safety of the blood supply. The proportion of vaccinated donors in $\mathrm{BC}$ will increase over time as a result of existing publicly funded HBV vaccination programs of grade six students since 1992 and infants since 2001.

\section{Student Posters Thursday, February 28 Pavilion Ballroom Foyer}

\footnotetext{
SP50

RAPID SNP PROFILING OF CRYPTOSPORIDIUM ISOLATES FROM SPORADIC CASES; A 5 CONTINENT STUDY

J Williamson*, C Ong

University of British Columbia, Vancouver, BC

OBJECTIVE: Identifying the degree of genetic diversity in an organism that is linked to the environment, such as Cryptosporidium, will aid in the understanding of its complex transmission dynamics. The aim of this study was to investigate the extent and nature of genetic variation between C. hominis and C. parrum by mapping and validating single nucleotide polymorphisms (SNPs) in bio-functionally relevant loci throughout the Cryptosporidium genome using isolates from sporadic cases in 5 different continents. Another objective of this study was to determine the efficacy by which unknown isolates could be typed using SNPs as a rapid method for species identification.

METHODS: Using the sequenced and annotated C. hominis and C. parvum genomes in silico data mining coupled to bioinformatics analysis was carried out to identify target proteins for mutation profiling. SNPs were typed
}

and validated using the SNaPshot SNP-typing ${ }^{\mathrm{TM}}$ system to analyze three target loci, Cp23, COWP and $\beta$-tubulin, from purified multiplex-PCR amplicons. Based on single base extension chemistry SNPs were identified through specifically designed capture probes unique to each target SNP. The multi-locus SNP-type (MLS) determined for each isolate was used to make species distinctions and identify variant or novel alleles at each specific SNP. Based on allele type, a mathematical input file of each MLS was created and sorted by geography. Using the Fstat ${ }^{\mathrm{TM}}$ Statistics software package, genetic variation was computed into a quantitative value with predicted measurements of total genetic diversity obtained. Isolates used in the study were collected from sporadic cryptosporidiosis cases in Canada, Australia, Kenya, Scotland and Peru.

RESULTS: A total of 91 isolates were typed and SNP profiles comprised of a total of 16 SNPs were compiled. Of the 5 SNPs typed from the Cp23 locus complete genetic stability was seen. In the $\beta$-tubulin locus 6 different instances of either C. hominis or C. parvum alleles replacing the anticipated profile was found. As well, mixed or double alleles were observed in multiple isolates from all 4 of the international populations for the S-BT3 SNP including a novel variant allele in a lone C. hominis isolate from Scotland. Although all 5 SNPs on the COWP locus showed an overall trend towards genetic stability, 1 SNP, S-COWP3, was a novel biallelic type with both C. hominis and C. parvum alleles present in 4 Australian and 2 Scottish isolates. As well, a single Peruvian isolate was also biallelic, with both C. hominis and C. parvum alleles at the S-COWP7 SNP. Statistical analysis of the SNP data suggests that genetic diversity within a population was significantly greater than between populations. Considering the diverse geographic locations involved in this study, it is likely that the environment may be a factor in mutation profiles. The presence of double alleles also calls attention to mixed infections being more prevalent than previously thought.

CONCLUSION: SNP profiling was found to be an efficient and reliable means of speciation with potential diagnostic applications to the identification of unknown isolates. We were able to rapidly type Cryptosporidium isolates from 5 different continental locations as well as ascertain their geographical genetic stability. This SNP validation study will be useful to cryptosporidiosis epidemiology as well as the development of more effective prevention and treatment strategies for different geographical areas.

\section{SP51}

\section{CANDIDEMIA: EPIDEMIOLOGY, RISK FACTORS, AND INCIDENCE OF MIXED CANDIDEMIA}

\section{G Al-Rawahi ${ }^{1 *}$, D Roscoe ${ }^{2}$}

'British Columbia Children's and Women's Hospital; ${ }^{2}$ Vancouver General Hospital, Vancouver, BC

BACKGROUND: Recent studies report differences in the epidemiology of blood stream infection (BSI) due to Candida species. This study was done to determine the epidemiology of Candida species BSI and associated risk factors in our Canadian tertiary care hospital.

METHODS: Patients with candidemia between January 2000 and December 2006 were identified using the microbiology laboratory database. A retrospective chart review was done to gather demographic data, underlying medical diagnoses and risk factor information.

RESULTS: A total of 231 patients were identified from the culture database. 62 patients were excluded because charts were not available for review. Of the 169 patients included in the final analysis, 59\% were males and $54 \%$ were more than 60 years old. The most common risk factors for candidemia were presence of a central venous line (72\%) and antibiotic use $(85 \%)$. The most frequent species isolated in the study period was C. albicans (51\%), followed by C. glabrata (31\%), C. parapsilosis (8\%), C. krusei (5\%), C. tropicalis (3\%), and C. lusitaniae and C. dubliniensis (1\% for each). C. albicans was the predominant species in all study years with the exception of 2002 where C. glabrata was more frequent. Ten patients (6\%) had mixed candidemia; 6 patients had C. albicans/C. glabrata, and 1 patient each had C. albicans/C. parapsilosis, C. albicans/C. dubliniensis, C. glabrata/C. parapsilosis and C. glabrata/C. tropicalis. Risk factors for mixed candidemia were not significantly different from those for monomicrobial candidemia, $80 \%$ vs. $65 \%$ for central venous line ( $p=0.67), 90 \%$ vs. $85 \%$ for antibiotic prophylaxis $(\mathrm{p}=1.0), 0$ vs. $10 \%$ for antifungal prophylaxis $(\mathrm{p}=0.54), 10 \%$ 
vs. $30 \%$ for abdominal surgery $(\mathrm{p}=0.37), 0$ vs. $30 \%$ for underlying malignancy $(\mathrm{p}=0.07)$.

CONCLUSION: C. albicans remains the most commonly isolated species in this setting, consistent with findings from other Canadian centres. Mixed candidemia does not appear to be higher in patients with identified risk factors.

\section{SP52 \\ CRYSTAL STRUCTURE OF THE F PLASMID TRAD-TRAM COMPLEX REVEALS A POTENTIAL DRUG TARGET FOR CONTROLLING TRANSMISSION OF THE MULTI-DRUG RESISTANCE MEDIATED BY F-LIKE R FACTORS}

J Lu*, J Wong, R Edwards, J Manchak, L Frost, J Glover

University of Alberta, Edmonton, AB

OBJECTIVE: Bacterial conjugation accounts for rapid acquisition of multi-drug resistance in many medically important human pathogens. Conjugative transfer of $\mathrm{F}$ plasmid DNA between bacteria requires interactions between a component of the DNA-protein complex (known as relaxosome), TraM, and the coupling protein $\mathrm{TraD}$, a hexameric ring ATPase that forms the cytoplasmic face of the conjugative pore. The objective of this study is to understand how $\operatorname{TraM}$ and $\operatorname{TraD}$ specifically interact at the atomic level and thus to find out a potential target for inhibiting F-mediated bacterial conjugation.

METHODS: TraM-TraD co-crystals were obtained using the hanging drop vapor diffusion technique. A native X-ray diffraction data set was collected at Advanced Light Source (ALS). The structure was solved by molecular replacement using the $\operatorname{TraM}^{58-127}$ structure as the search model in MOLREP. Structure-based mutagenesis and bacterial mating assays were used to test the importance of TraM-TraD interactions in vivo.

RESULTS: The crystal structure of the TraM tetramerization domain bound to the C-terminal tail of $\mathrm{TraD}$ was solved at $2.5 \AA$ resolution. This is the first structural evidence of a relaxosome-coupling protein interaction. The structure reveals four symmetry-related grooves on the surface of the TraM tetramer that each bind a single TraD peptide. These interactions involve the TraD C-terminal phenylalanine side chain and mainchain carboxylate, as well as long-range electrostatic interactions, which are necessary for efficient conjugation in vivo. Our results suggest that these interactions are important for initial, specific contacts between the relaxosome and the coupling protein that lead to more generalized interactions that stabilize the relaxosome-coupling protein complex in preparation for conjugative DNA transfer.

CONCLUSION: This work demonstrates a direct and specific recognition of the C-terminal carboxylate of the flexible TraD tail. Comparisons with other systems indicate that similar interactions may govern signal sequence recognition in other type IV secretion systems of various bacterial pathogens. Our results present a new target for the development of agents which could control the spread of multi-drug resistance mediated by the conjugative transfer of F-like R-factors.

\section{SP53}

VALIDATION OF PYRROLIDONYL ARYLAMIDASE (PYR) IN COMBINATION WITH ORNITHINE DECARBOXYLASE (ODC) FOR IDENTIFICATION OF STAPHYLOCOCCUS LUGDUNENSIS (LUG)

DHS Tan 1,3*, BM Willey 1,2, P Akhavan ${ }^{1}$, L Stoakes ${ }^{4}$, S Abood ${ }^{1}$, L Mazzulli ${ }^{1}$, G Small1,2, SM Poutanen ${ }^{1,2,3}$

${ }^{1}$ Mount Sinai Hospital; ${ }^{2}$ University Health Network; ${ }^{3}$ University of Toronto, Toronto; ${ }^{4}$ London Health Sciences Centre, London, ON OBJECTIVES: LUG is a virulent coagulase-negative staphylococcus (CNST) with different CLSI breakpoints compared to other CNST. Unlike other CNST, LUG is characteristically positive (+) for PYR and ODC. However, PYR kits have not yet been validated for testing with staphylococci. This study evaluated PYR kits from 5 manufacturers, and together with ODC activity, assessed their ability to differentiate LUG from other CNST.

METHODS: A total of 129 CNST (64 PYR+: 52 LUG, 10 S. schleiferi, 2 S. haemolyticus; 65 PYR-negative: 4 S. hominis, 56 S. epidermidis, 3 S. warneri,
$1 \mathrm{~S}$. pasteuri, $1 \mathrm{~S}$. capitis) that had been identified by fatty acid, $16 \mathrm{~S}$ rRNA sequencing and/or conventional biochemicals, were selected for the study. These isolates were tested for PYR by BBL DrySlide (BD), Hardy, MedOx, OBIS (Oxoid) and Remel kits, and for ODC activity using the Roscoe Diagnostic Tablets (ProLab). PYR reactions were recorded as + (strong), negative (no colour change), or indeterminant (weak) at $1 \mathrm{~min}$ (20 sec for OBIS) by 3 readers blinded to CNST species identification and to each other's results. Results were inconclusive if there was disagreement between readers or a consensus that reactions were indeterminant. Sensitivities and specificities excluded inconclusive results.

RESULTS: Of 52 LUG, 100\% were PYR+/OCD+ by all kits, except 1 PYR-inconclusive and ODC+ result by MedOx. No other CNST species was PYR+/ODC+. The sensitivity for LUG with PYR/ODC combined was $100 \%$ (95\% CI 92-100) for all PYR kits and specificity was 100\% (95\% CI: BD 93-100, Hardy 75-100, MedOx 72-100, OBIS 73-100, Remel 93-100). Inter-reader agreement (kappa) was similar among PYR kits: BD 0.87, Hardy 0.97, MedOx 0.82, OBIS 1.0, Remel 0.90 . The $\%$ of reads that were inconclusive per kit were: BD $15 \%$ (95\% CI 10-22), Hardy $49 \%$ (40-57), MedOx 51\% (43-60), OBIS 50\% (41-58), and Remel 12\% (8-19). Most inconclusive results were noted with PYR-negative CNST species.

CONCLUSIONS: The use of combined PYR+/ODC+ reactions with any PYR kit was highly accurate in separating LUG from other CNST. However, for PYR kits other than BD and Remel, there were an unacceptable numbers of inconclusive results, limiting their usefulness. Considering weak PYR reactions for CNST as negative will minimize inconclusive results without significantly affecting the ability to detect LUG for all kits.

\section{SP54}

DURATION OF EFFECTIVE MRSA DECOLONIZATION: A RETROSPECTIVE COHORT ANALYSIS

J Zheng*, C Lemieux, M Gardam

Infection Prevention and Control Unit, University Health Network, Toronto, ON

WITHDRAWN

\section{SP55}

MEDIASTINITIS DESPITE APPROPRIATE PROPHYLAXIS IN CARDIAC SURGERY: WHAT ELSE SHOULD WE BE DOING TO PREVENT INFECTION?

RA Harrison*, LM Saxinger, G Taylor

University of Alberta, Edmonton, AB

OBJECTIVE: To describe pre- and post-operative findings in patients with organ space (OS) mediastinal infections (mediastinitis) at a Canadian tertiary care hospital.

METHODS: Patients identified in the hospital prospectively collected Infection Control database who had cardiac surgery complicated by OS infection (as defined by CDC) between January 1, 2000 and December 30, 2005 were included. Data derived from Infection Control data sheets and patient chart review were analyzed. Patient, peri-operative, and postoperative factors were reviewed, and outcome measures included average length of the first hospital admission, and death prior to discharge.

RESULTS: Of 7632 cardiac surgery patients surveilled, 78 had OS infection (1 per 100 surgeries); 54/78 (69\%) were after coronary bypass surgery, and $24 / 78(31 \%)$ followed heart valve replacement or repair, with or without coronary bypass. The average length of first hospital admission was 25 days (range 3-142), and death occurred during the first admission in 10\% of cases. All infections involved Gram positive organisms, predominantly Staphylococcus sp. (90\%): 38 S. aureus (2 MRSA ), and 32 coagulase negative Staphylococcus sp. The mean age of patients with infection was 66 (range 21-89); the mean body mass index was 27.5 (range 21-45), 54\% of patients had diabetes mellitus, and $21 \%$ smoked pre-operatively. Appropriate (drug and timing of administration) of pre-operative antibiotic prophylaxis was administered in $99 \%$ of cases, although repeated doses of antibiotic intraoperatively were rare $4 / 37$ (10\%), and dosing was inconsistently adjusted for elevated body mass index.

CONCLUSION: Previously described risk factors remained relevant in 
this review. In the majority of cases, pre-operative antibiotics were administered according to established guidelines, yet Gram positive organisms were isolated from every mediastinal infection over a 6 year period. This highlights the need to consider novel targeted preventive measures, and the importance of ongoing surveillance, particularly in an era of increasing Gram positive organism resistance.

\section{SP56}

IMPLEMENTATION OF A HUMAN PAPILLOMAVIRUS NEUTRALIZING ANTIBODY ASSAY BASED ON PSEUDOVIRIONS

S So ${ }^{1 *}$, K Karunakaran ${ }^{1}$, J Palefsky ${ }^{3}$, M Krajden ${ }^{1,2}$, M Petric ${ }^{1,2}$

${ }^{1} \mathrm{BC}$ Centre for Disease Control; ${ }^{2}$ University of British Columbia,

Vancouver, BC; ${ }^{3}$ University of California San Francisco, San

Francisco, CA, United States

OBJECTIVES: The methods of Buck et al. (2007) were used to generate HPV 16 and 18 pseudovirions consisting of the L1 and L2 coat proteins and a secreted embryonic alkaline phosphatase (SEAP) reporter gene in a plasmid vector. These virus-like particles (VLPs) are designed to be used in a neutralizing antibody assay to measure antibody levels to HPV 16 and 18 following immunization.

METHODS: VLPs containing the SEAP reporter gene were generated in 293TT cells transfected with the respective plasmids containing genes L1 and L2 to HPV 16 or 18 together with a plasmid encoding SEAP. VLP's were purified by isopycnic density gradient ultracentrifugation and the presence of virus structures was confirmed by electron microscopy. Type specific serological reactivity was confirmed by Western Blot using rabbit sera reactive to HPV 16 L1 and HPV 18 L1. Infection of 293TT cell monolayers with pseudovirion preparations resulted in elevation in the level of SEAP in the medium whose activity on a CSPD substrate could be measured by light emittance in relative light units (RLU). Neutralizing antibody to the respective virus would inhibit the infection and preclude the appearance of SEAP activity in the medium.

RESULTS: Infectivity of 293TT cells by the VLPs was readily detectable by measuring SEAP activity. After purification, the pseudovirus HPV 16 VLP titre was approximately $10^{-8}$ and the HPV 18 VLP titre was approximately $10^{-4}$ infectious units per $\mathrm{mL}$. Both preparations are adequate for use in neutralizing antibody assays. The neutralizing antibody assay was in principle confirmed by assessing pseudovirus neutralization with heparin sodium salt, an established surrogate known to neutralize VLP infectivity. Concentrations of heparin above $7.81 \mu \mathrm{g} / \mathrm{ml}$ were able to neutralize HPV VLP 16.

CONCLUSIONS: These VLP preparations can now be used in large scale serological studies. Validation will be based on the use of internationally standardized sera, comparison with the Merck Luminex based HPV type specific assay and an assessment of serological panels of HPV vaccinated and non-vaccinated controls.

\section{Poster Presentations Thursday, February 28 Pavilion Ballroom Foyer}

P1

IDENTIFICATION OF NUTRITIONALLY VARIANT

STREPTOCOCCI AND AEROBIC ACTINOMYCETES AS PUTATIVE TARGETS FOR VERIFICATION BY 16S rDNA SEQUENCING METHODOLOGIES

SJ Drews ${ }^{1,2,4 *}$, C Delima ${ }^{4}$, P Rawte ${ }^{4}$, S Brown ${ }^{4}$, S Richardson ${ }^{1,2,4}$, F Jamieson ${ }^{1,2,3}$

${ }^{1}$ Department of Laboratory Medicine and Pathobiology, University of Toronto, Toronto, ON; ${ }^{2}$ Mount Sinai Hospital, Toronto, ON; ${ }^{3}$ Hospital for Sick Children, Toronto, ON; ${ }^{4}$ Ontario Public Health Laboratories, Toronto, ON

OBJECTIVE: There are no uniform standards for the interpretation of $16 \mathrm{~S}$ ribosomal DNA ( $\mathrm{rDNA}$ ) sequence data and the assignment of bacterial isolates into specific taxonomic groupings. This study will identify candidate bacteria for further verification studies utilizing a $16 \mathrm{~S}$ rDNA-based algorithm and a publicly available sequence database.

METHODS: Unidentified bacterial isolates were sent for rDNA sequencing analysis of a 700-800 nucleotide region. Query sequences were compared to the NCBI database. Significant alignments for analysis required the following criteria: 1) minimum length $500 \mathrm{bp}, 2$ ) deposition into database on or after 1995, 3) peer-reviewed publication or submission from $>1$ independent laboratory. Taxonomic identification required; 1 ) $\geq 99 \%$ identity to determine species, or 2) $\geq 97 \%$ identity to determine genus, and 3 ) identification to the most common taxonomic level. Identification based on sequence was then compared to key phenotypic tests as follows; 1) Gram stain, 2) Culture morphology, and 3) Key biochemical/phenotypic results for the level of sequence identity as outlined in the "Manual of Clinical Microbiology," other reference texts or peer reviewed publications.

RESULTS: 213 isolates were sent for 16S rDNA sequencing. Identification to a species level was possible in 79/213 (37\%) of isolates. Identification to a genus level was possible in $82 / 213$ (38\%) of isolates. Identification to a family group or higher was possible in 14/213 of isolates (7\%). Two of the most prevalent groups identified by $16 \mathrm{~S}$ rDNA were the nutritionally variant streptococci $(35 / 213,16 \%)$ and aerobic actinomycetes $(33 / 213,15 \%)$. Of the difficult to identify isolates identified as nutritionally variant streptococci by sequencing, 31/35 (89\%) had phenotypic results consistent with genotypic identification. Of the difficult to identify isolates identified as aerobic actinomycetes, only 16/33 (48\%) had phenotypic results consistent with genotypic results.

CONCLUSIONS: This preliminary study indicates that nutritionally variant streptococci may be rationale targets for future verification studies that primarily utilize $16 \mathrm{~S}$ rDNA for bacterial identification. The poor association of genotype and phenotype for difficult to identify aerobic actinomycetes indicates that further verification studies should utilize multiple genetic targets for the sequence-based identification of these organisms.

P2

\section{BIOFILM FORMATION POTENTIAL OF STAPHYLOCOCCI ON POLYMER SURFACE AND ITS CO-RELATION WITH METHICILLIN SUSCEPTIBILITY}

A Chaudhury*, A Gururajkumar

SV Institute of Medical Sciences, Tirupati, Andhra Pradesh, India INTRODUCTION: Biofilm production in Staphylococci depends to a large extent on the production of a polysaccharide intercellular adhesin (PIA). Preliminary studies suggest that there may be significant differences in the mechanism and regulation of biofilm formation in methicillin sensitive (MSSA) and -resistant (MRSA) S. aureus. Various environmental factors like glucose, $\mathrm{NaCl}$, etc have been shown to influence the ica operon expression needed for PIA production. In this study we have examined the effect of methicillin susceptibility and the influence of $\mathrm{NaCl}$ and glucose 
on biofilm formation on polymer surface by $S$. aureus and S. epidermidis. METHODS: A total of 60 representative strains consisting of 15 each of MRSA, MSSA, methicillin sensitive S. epidermidis (MSSE), and methicillin resistant $S$. epidermidis (MRSE) isolated from venous catheters were studied. The ability to form biofilm was studied on 96 wells polystyrene plates and were read at $490 \mathrm{~nm}$. An optical density of $>0.120$ was considered as biofilm positive.

RESULTS: Among the S. aureus isolates, none of the MSSA produced biofilms with or without glucose, while 2 strains could do so in $\mathrm{NaCl}$. For MRSA, 6 strains showed this activity by itself, with additional 4 strains (total 10) and one strain in the presence of glucose and $\mathrm{NaCl}$ respectively. Only one strain each of MSSE and MRSE could produce biofilm without any external stimulus. For MSSE, biofilm formation was positive for additional one strain in $\mathrm{NaCl}$ and two in glucose. The lone MRSE isolate was positive for biofilm in glucose, but not in $\mathrm{NaCl}$.

DISCUSSION: In our study, a greater number of MRSA could show biofilm formation compared to the other three groups, i.e. MSSA, MSSE, and MRSE. These three groups showed very limited potential to form biofilm on smooth surfaces even in the presence of glucose or $\mathrm{NaCl}$. Although the isolates were from plastic catheters, they showed varying ability in their adherence to smooth polystyrene surface in vitro. The situation is more complex in the inserted catheters which become coated with various host proteins and the organisms can adhere with the help of their own surface proteins. Duplication of in vivo conditions may give a more realistic picture of the whole process.

\section{P3}

IMPACT OF 7-VALENT PNEUMOCOCCAL CONJUGATE VACCINE (PCV7) ON SEROTYPE DISTRIBUTION OF STREPTOCOCCUS PNEUMONIAE (SPN) CLINICAL ISOLATES IN CANADA

J Gubbay ${ }^{2 *}$, A McGeer ${ }^{1}$, K Green ${ }^{1}$, S Pong-Porter ${ }^{1}$, D Low ${ }^{1}$

${ }^{1}$ Canadian Bacterial Surveillance Network (CBSN), Mount Sinai Hospital, Toronto, ON; ${ }^{2}$ Ontario Public Health Laboratories, Toronto, ON

OBJECTIVE: In 2002, PCV7 was introduced into the routine childhood vaccination schedule in some Canadian provinces and territories, with most including it by 2005. Recent studies have documented an increased relative incidence of non-vaccine serotypes (NVS) since the introduction of PCV7, in particular 19A. The objective of this study was to document the change in serotype distribution of SPN isolates in Canada following introduction of PCV7.

METHODS: A selection of SPN isolates pre (1993 to 2001) and post (2002 to 2007) introduction of PCV7 were serotyped and compared. Sterile (SI) and nonsterile site isolate (NSI) serotype distributions were also analyzed for children $<2$ years, adults $\geq 65$ years of age, and ciprofloxacin resistant (CR) (MIC $\geq 4 \mu \mathrm{g} / \mathrm{ml})$ organisms.

RESULTS: The proportion of SPN vaccine serotypes (VS) dropped from $69.3 \%$ (4087 serotyped) pre to $50.3 \%$ (5499 serotyped) post PCV7 $(\mathrm{P}<0.0001)$, with the largest reduction in serotype $14(17.4 \%$ to $8 \%$, $\mathrm{P}<0.0001)$. There was no change in the serotype distribution among CR isolates. Among SI, there was a fall in VS from $66.8 \%$ to $52.4 \%$ $(\mathrm{P}<0.0001)$, with an increase in NVS 6A $(3.8 \%$ to $5.5 \%, \mathrm{P}<0.0001)$. Among NSI, VS fell from $75.8 \%$ to $48.2 \%$ ( $\mathrm{P}<0.0001)$. Among children $<2$ yo, there was a drop in VS isolates $(84.4 \%$ to $77.8 \% ; \mathrm{P}=0.0013)$ and an increase in NVS 19A (3.4\% to $9.8 \%, \mathrm{P}<0.0001)$. This $19 \mathrm{~A}$ increase was greater in NSI $(4.8 \%$ to $11.9 \%, \mathrm{P}=0.0064)$ than SI $(2.5 \%$ to $7.9 \%, \mathrm{P}=$ $0.0018)$. Among persons $\geq 65$ years of age, VS fell $(61.5 \%$ to $44.8 \%$, $\mathrm{P}<0.0001$ ). Of SPN isolates since 2002, the 9-valent and 13-valent PCVs would cover $50.3 \%$ and $70.1 \%$ of all isolates, respectively, and $52.5 \%$ and $72.0 \%$ of SI, respectively.

CONCLUSIONS: Introduction of PCV7 in Canada has resulted in a proportionate decrease in VS SPN isolates, which is greater among NSI. The decrease is almost exclusively among $\mathrm{C}$ sensitive and not CR SPN. We have also documented a proportionate increase in NVS 19A in children $<2$ years of age. A 13 -valent PCV would cover $20 \%$ more of the current SPN isolates than PCV7, but a 9-valent PCV would provide no additional serotype cover.

\section{P4}

EVOLUTION OF PARC AND GYRA QUINOLONERESISTANCE DETERMINING REGION (QRDR) MUTATIONS IN FLUOROQUINOLONE RESISTANT (FQR) STREPTOCOCCUS PNEUMONIAE (SPN) FOLLOWING INTRODUCTION OF MOXIFLOXACIN (MOXI) IN CANADA J Gubbay ${ }^{2 *}$, A McGeer ${ }^{1}$, K Green ${ }^{1}$, R Melano ${ }^{2}$, C Seah ${ }^{2}$, C Duncan ${ }^{1}$, S Pong-Porter ${ }^{1}$, S Drews ${ }^{2}$, D Low ${ }^{1,2}$

${ }^{1}$ Canadian Bacterial Surveillance Network, Mount Sinai Hospital, Toronto, ON; ${ }^{2}$ Ontario Public Health Laboratories, Toronto, ON BACKGROUND: Since the introduction of Moxi in 2000 (which preferentially targets gyrA), it has replaced ciprofloxacin (C) and levofloxacin (L) (both which preferentially target parC) as the most frequently used FQ used for the treatment of respiratory tract infections in Canada. This study examines changes in frequency of mutations in parC and gyr A only in FQR $\mathrm{SPN}$, as a measure of which agents may be driving resistance.

METHODS: Mutations in the QRDR of $\operatorname{parC}$ and $g y r A$ were determined in all isolates with a C MIC $\geq 4 \mu \mathrm{g} / \mathrm{ml}$ and a Moxi MIC $\geq 0.5 \mu \mathrm{g} / \mathrm{ml}$ from 1994-2000 and 2001-2006. Proportions were compared using $\chi^{2}$ or Fisher's Exact test.

RESULTS: C resistance (R) increased from $1.25 \%$ (of 12685 isolates) to $2.25 \%$ (of 14581 isolates), Moxi R ( $\mathrm{MIC} \geq 4 \mu \mathrm{g} / \mathrm{ml}$ ) increased from $0.19 \%$ (of 1551 isolates) to $0.72 \%$ (of 2505 isolates), and isolates with elevated Moxi MIC $\geq 0.5 \mu \mathrm{g} / \mathrm{ml}$ (MEL) increased from $0.77 \%$ to $1.96 \%$. parC only mutations decreased significantly in CR. gyrA only mutations increased in a non-significant manner in CR and MEL. parC+gyrA mutations increased significantly in CR.

\begin{tabular}{lcccccc} 
& \multicolumn{3}{c}{ C MIC $\geq \mathbf{4} \mathbf{\mu g} / \mathbf{m l}$} & \multicolumn{4}{c}{ Moxi MIC $\geq \mathbf{0 . 5} \mathbf{\mu g} / \mathbf{m l}$} \\
& $\mathbf{1 9 9 4 - 2 0 0 0}$ & $\mathbf{2 0 0 1 - 0 6}$ & $\mathbf{p}$ & $\mathbf{1 9 9 4 - 2 0 0 0}$ & $\mathbf{2 0 0 1 - 0 6}$ & $\mathbf{p}$ \\
Mutations & $\mathbf{n ~ ( \% )}$ & $\mathbf{n}(\%)$ & value & $\mathbf{n}(\%)$ & $\mathbf{n}(\%)$ & value \\
\hline parC only & $74(51 \%)$ & $79(29 \%)$ & $<0.0001$ & $1(10 \%)$ & $8(30.8 \%)$ & 0.39 \\
gyrA only & $2(1.4 \%)$ & $5(1.8 \%)$ & 1 & $1(10 \%)$ & $4(15.4 \%)$ & 1 \\
parC+ gyrA & $56(38.6 \%)$ & $172(63.2 \%)$ & $<0.0001$ & $8(80 \%)$ & $13(50 \%)$ & 0.14 \\
\hline
\end{tabular}

CONCLUSION: There has been a significant decrease in the frequency of parC only mutations but not a concomitant increase in gyrA only mutations, suggesting Moxi is not driving FQ resistance.

\section{P5}

\section{SELECTION OF HIGH LEVEL TELITHROMYCIN RESISTANCE IN ERYTHROMYCIN RESISTANT GROUP A (GAS) AND GROUP B (GBS) STREPTOCOCCUS}

S-B Harvey ${ }^{1,2 *}, K_{\text {Ramotar }}^{1,2}, M$ Desjardins ${ }^{1,2}$

${ }^{1}$ The Ottawa Hospital, Ottawa, ON; ${ }^{2}$ The Ottawa Hospital Research Institute, Ottawa, ON

BACKGROUND: In Eastern Ontario 17\% and $8 \%$ of GBS and GAS are erythromycin resistant. Constitutive and inducible erm mediated resistance was identified in $46 \%$ and $54 \%$, respectively, of erythromycin resistant GBS and $64 \%$ and $36 \%$, respectively, of GAS. Erythromycin resistance in the remaining isolates was mediated by efflux (mefA). Although telithromycin resistance has been reported in erythromycin resistant GAS, this antibiotic still remains an active therapeutic alternative. We determined telithromycin activity in erythromycin resistant GBS and GAS and selected for high-level resistance in isolates with inducible erm.

METHOD: A total of 55 and 49 genotypically characterized erythromcyin resistant GBS and GAS, respectively, were tested against telithromycin by broth microdilution according to CLSI recommendations. Selection of high-level telithromycin resistance in 3 GBS and 2 GAS with inducible erm was performed by serial passage on plates containing increasing doubling concentrations of telithromycin. The attenuator and coding region of the erm gene and the 6 alleles of domain V of the 23s RNA of each strain was sequenced and compared before and after passage. Induced telithromycin MICs were determined for GBS and GAS with inducible (26 GBS and 7 GAS) and constitutive erm (32 GBS and 44 GAS) by adding $4 \mu \mathrm{g}$ or $16 \mu \mathrm{g}$ erythromycin in each well of the microdilution plates. 
RESULTS: Telithromycin MIC 90 for GAS was $64 \mu \mathrm{g} / \mathrm{mL}$ (1 isolate with $64 \mu \mathrm{g} / \mathrm{mL}$ ) and $0.5 \mu \mathrm{g} / \mathrm{mL}$ for GBS. Irreversible high level telithromycin resistance (MIC $\geq 128 \mu \mathrm{g} / \mathrm{mL}$ ) was selected for in all GAS and GBS within 12 to 14 passages. No mutations were identified in the erm or domain $\mathrm{V}$ genes for 2 of 3 GBS and both GAS. For one GBS, a single point mutation (Asp to Ser) was identified at base pair 332 of the erm gene. Erythromycin induction increased the telithromycin MIC more than 8 fold from $0.5 \mu \mathrm{g} / \mathrm{mL}$ to $\geq 128 \mu \mathrm{g} / \mathrm{mL}$ in isolates with either constitutive or inducible erm.

CONCLUSION: Telithromycin remains an effective alternative to betalactams in erythromycin resistant GBS and GAS. Although the mechanism of telithromycin resistance in GBS and GAS remains unclear, repeated exposure to telithromycin could select for high-level resistance. Changes in erm methylation efficiency alone or in combination with other mechanisms could potentially lead to selection and dissemination of isolates with high-level resistance.

\section{P6}

COMPARISON OF CULTURE MEDIA AND METHODS USED FOR THE DETECTION OF GROUP B STREPTOCOCCUS (GBS) FROM PRE-NATAL VAGINAL/RECTAL SPECIMENS

P Kornherr*, K Campbell, D Dowhaniuk, D Raymondo, A Hosselet, S Valupadas, D McLean

Gamma-Dynacare Laboratories, Ottawa, ON

OBJECTIVE: The CDC recommended that laboratories maximize recovery of GBS by using a selective enrichment broth and then sub-culturing to blood agar. Attention to atypical GBS colonies is also recommended. Because diverse bacteria from these samples may appear similar to and may have inhibitory effects on GBS growth, detection may prove difficult. This study investigates A) Todd Hewitt Broth with blood (gent. $8 \mathrm{mcg} / \mathrm{ml}$.) vs. Modified THB (gent. $14 \mathrm{mcg} . / \mathrm{ml}$.) by direct grouping and sub-culturing, B) hemolysis and growth on 5\% sheep blood agars (TSA and Columbia) from 2 manufacturers, and C) an enhanced technique incorporating a $10 \mathrm{mcg}$. gentamicin disc, for the purpose of detecting GBS.

METHODS: A total of $218 \mathrm{vag} / \mathrm{rectal}$ swabs for GBS screening were immersed in saline, vortexed and a challenging $50 \mathrm{muL}$ distributed to each of 1) a CNA plate, 2) standard THB (STHB) and 3) modified THB (MTHB). After overnight incubation, broths were grouped directly using PathoDx and subbed to 1)TSA and 2)Columbia blood agars (Oxoid and PML). A gentamicin disc was placed in the $2^{\text {nd }}$ quadrant of each sub plate. Comparative studies of sub growths, direct grouping and usefulness of the gent.disc were performed.

RESULTS: 88 positive GBS were recovered via any 1 or combination of tests. 72\% positive GBS were recovered from initial CNA, 90\% from STHB and $94 \%$ from MTHB subs (combined 100\%) using Oxoid TSA and Columbia. PML TSA recovered 5\% lower results but PML Columbia recovered approximately $20 \%$ less based on a lack of hemolysis. The gent disc assisted detection up to $20 \%$ due to its demonstration of GBS' ability to grow up to its edge whereas most other bacteria formed a zone of inhibition. This enhanced the recovery of non-hemolytic GBS. PathoDx direct broth grouping gave $96 \%$ sens $/ 100 \%$ spec.

CONCLUSIONS: GBS hemolysis quality was: Oxoid TSA>Oxoid Columbia $=$ PML TSA $>$ PML Columbia. PathoDx direct grouping has high sens and spec. and can improve turn around time. MTHB recovery was slightly better than STHB. TSA agars gave the visual impression that normal flora was suppressed when compared to Columbia. Adding gent discs to subcultures can assist GBS detection especially when A) GBS and E. faecalis are present and/or B) in the absence of GBS hemolysis. Nonhemolytic GBS ranged from 3\% to 20\% depending on media used. Blood hemolysis in the broth was useful for screening positives.

\section{P7 \\ DEVELOPMENT OF A NOVEL CHIMERIC ANTHRAX SEROLOGY STANDARD}

C Corbett ${ }^{1,2 *}$, L Schmidt ${ }^{1}$, V Semenova ${ }^{3}$, J Schiffer ${ }^{3}$, D Boese ${ }^{2}$, C Quinn ${ }^{3}$, J Berry ${ }^{1,2}$

${ }^{1}$ National Microbiology Laboratory, Public Health Agency of Canada, Winnipeg, MB; ${ }^{2}$ Department of Medical Microbiology and Infectious Disease University of Manitoba, Winnipeg, MB; ${ }^{3}$ Centers for Disease Control and Prevention, Atlanta, GA, United States

OBJECTIVE: The current anthrax serology human standard reference serum AVR801 is derived from serum of healthy adults who have been vaccinated with the Anthrax Vaccine Adsorbed (AVA). It is desirable to develop an unlimited source of a homogeneous reagent for use as a serological standard for anthrax. Herein, we describe the development and testing of a human-murine chimeric antibody for use as a novel anthrax serology standard.

METHODS: The human-murine chimeric monoclonal antibody (huG1F20G75) was previously developed using a two vector system for expression of whole chimeric IgG1. To determine if huG1-F20G75 could be used as a serological control for anthrax, it was compared to AVR801. For routine anthrax serology AVR801 is serially diluted in parallel to the patient sample and run in an indirect ELISA using PA $(2.0 \mu \mathrm{g} / \mathrm{ml})$ as the coating antigen. The total amount of anti-PA IgG antibodies present in the clinical sample is interpolated from the standard curve generated using AVR801. A sample is considered non-reactive for anthrax serology if the concentration of anti-PA IgG antibodies is lower than the lower limit of detection for the assay (typically $<3 \mu \mathrm{g} / \mathrm{ml}$ ).

RESULTS: When the anti-PA chimeric mAb, huG1-F20G75 was serially diluted to create a standard curve to interpolate the concentration anti-PA antibodies in AVR801 a concentration of $171 \mu \mathrm{g} / \mathrm{ml}$ was obtained, whereas the assigned anti-PA IgG concentration for AVR801 is $109.4 \mu \mathrm{g} / \mathrm{ml}$. The differences we observe in the concentrations of anti-PA antibodies may be due to inherent inaccuracies in quantitation of the huG1-F20G75 via the BCA protein assay. In reverse, when the concentration of active huG1F20G75 was interpolated from the AVR801 standard curve, and this concentration was used for huG1-F20G75, comparable results were observed for the quantitation of anti-PA IgG in unknown serum for both huG1F20G75 and AVR801 serological controls. Numerous comparative assays have been conducted and we repeatedly obtain similar anti-PA concentrations utilizing huG1-F2075 and AVR801 as serological controls.

CONCLUSION: The recombinant chimeric monoclonal antibody demonstrates promise as an anthrax serological standard. This reagent may provide a potential unlimited source of a well-defined anthrax serological standard.

\section{P8}

\section{LIGHTCYCLER DETECTION OF MUMPS RNA BY REAL-TIME ONE-STEP REVERSE-TRANSCRIPTASE PCR (RT-PCR)}

J LeBlanc*, J Pettipas, S Campbell, R Davidson, T Hatchette QE II Health Sciences Centre, Halifax, NS

OBJECTIVE: Recent outbreaks worldwide highlight the need for rapid and accurate methods for mumps diagnosis. Real-time RT-PCR is a promising alternative to conventional PCR, although most assays lack an internal control and none have been validated for use on a LightCycler. In this report, we describe an internally-controlled real-time one-step RT-PCR assay for the detection of mumps RNA in clinical samples on the LightCycler platform and directly compare its use against a traditional RTPCR mumps assay. In addition, since repeated freeze/thaw cycles are known to hamper viral culture, we investigated its influence on RT-PCR. METHODS: Extraction of total nucleic acid was performed on the Roche MagNA Pure LC. Nested RT-PCR was performed as described in Jin et al. (1999). Real-time RT-PCR was performed on a LightCycler using primers and probes targeting $\mathrm{F}$ gene and RNaseP. The sensitivity of both assays was assessed by serial dilution of clinical specimens and plasmid controls. The specificity was tested against a number of viruses and mumps genotypes. Parallel testing was performed on 477 specimens consisting of oral swabs, urine and CSFs. For freeze/thaw experiments, clinical specimens were 
divided into aliquots and subjected to multiple freeze/thaw cycles prior to extraction and both conventional and real-time RT-PCR. After 7 cycles, RT-PCR was performed.

RESULTS: Our real-time RT-PCR assay was able to detect various mumps genotypes without cross-reaction with other viruses. Moreover, our assay was as sensitive as nested RT-PCR, with a lower detection limit of 1-10 copies. Of 477 clinical specimens (124 positive and 353 negative) evaluated in parallel, only one discordant result was observed. Conventional RT-PCR was far more labour intensive and time consuming than real-time RT-PCR. The average cost per specimen (without labour) was $\$ 15.60$ for conventional nested-PCR and $\$ 9.31$ for real-time RT-PCR. Despite 7 freeze-thaw cycles, all samples tested were positive by real-time and nested RT-PCR.

CONCLUSIONS: Our assay is sensitive, specific, and more rapid than conventional PCR. In addition it is significantly cheaper and less labour intensive making it a suitable method for diagnosis of mumps. In addition, the freeze/thaw analyses provide valuable information regarding storage and transport of specimens submitted for testing, particularly for laboratories in remote areas.

\section{P9}

\section{GRANULOMATOUS CERVICOFACIAL LYMPHADENITIS - NOT THAT MANY?}

KC Thoon*, CY Chong, NWS Tee

KK Women's and Children's Hospital, Singapore, Singapore

AIMS: Granulomatous cervicofacial lymphadenitis (GCL) is not uncommon in children, and often presents a diagnostic and management challenge. We sought to determine the epidemiologic profile of these patients and identify features that aid in management.

METHODS: We retrospectively analyzed data in our patients from 1998 to 2006 who had lymph node biopsies from the cervicofacial region that showed granulomatous lymphadenitis. Data was collected to describe the distribution of cases by age, sex, race, and clinical, laboratory, imaging and histopathologic characteristics. We also performed subgroup analysis for patients with positive lymph node cultures and for patients with and without recurrence after treatment.

RESULTS: We identified 60 children during this period with GCL, noting a rising incidence from 2003 onwards (>75\% cases after 2003). Mean age was 67 months, mean duration of symptoms before presentation was 7 weeks. Most presented with single (73\%) and unilateral (97\%) lymphadenopathy, located in the submandibular or pre-auricular/ parotid region $(55 \%)$, and had no features suggestive of acute bacterial lymphadenitis. Most (94\%) had a Tuberculin Skin Test (TST) reading of $<20 \mathrm{~mm}$. There were $10(19 \%)$ positive node cultures, including 7 isolates of Non-Tuberculous Mycobacteria (NTM; 4 Haemophilum, 2 Fortuitum, 1 not further identified), 2 isolates of M. Tuberculosis (TB), and 1 of $M$. Bovis (identified to be BCG (Bacille Calmette-Guérin) strain). Subgroup analysis suggested that patients with NTM GCL (compared to TB GCL) were younger (56 months vs. 99 months) and had multiple lymph nodes in the parotid/ pre-auricular or submandibular regions, but did not reach significance. Analysis with regards to recurrence after initial treatment revealed that younger age predicted recurrence (mean 42 months vs. 73 months in those who don't recur, $\mathrm{p}=0.05$ ) while initial complete excision of affected nodes predicted no recurrence ( $8 \%$ vs. $43 \%$ in those who with incomplete excision, $\mathrm{p}=0.03$ ).

CONCLUSIONS: The incidence of GCL appears to be rising. While microbiological yield is low, most appear to be due to NTM (instead of TB traditionally); our isolates were predominantly M. haemophilum or fortuitum. Initial complete excision of affected nodes (if possible) remains the best mode of treatment for these patients.

\section{P10}

HOSPITAL-ACQUIRED PNEUMOCOCCAL BLOODSTREAM INFECTION

C Pienaar ${ }^{*}$, SW Smith ${ }^{2}$, GJ Tyrrell ${ }^{3}$, M Lovgren ${ }^{3}$, GD Taylor ${ }^{2}$

${ }^{1}$ LifeLabs, Burnaby, BC; ${ }^{2}$ Department of Medicine, University of

Alberta, Edmonton, AB; ${ }^{3}$ National Centre for Streptococcus,

Provincial Laboratory for Public Health, Edmonton, AB

OBJECTIVES: Streptococcus pneumoniae is a frequent cause of community-acquired (CA) bloodstream infection (BSI) and risk factors are well established. The characteristics of hospital-acquired (HA) pneumococcal BSI are less well defined. We wished to determine risk factors associated with HA pneumococcal BSI, the serotypes of isolates and if these serotypes were included in the 7-valent conjugate vaccine (PCV7) and/or 23-valent polysaccharide vaccine (PPV23), as well as penicillin susceptibility.

METHODS: Data prospectively collected from 2000 to 2007 by Infection Control Practitioners who monitor blood culture reports and use the CDC definition to determine if BSI is HA i.e. isolation of S. pneumoniae from one or more blood culture(s) three or more days after hospital admission was reviewed. Risk factors were retrospectively identified by reviewing the medical records of patients. S. pneumoniae was identified by gram stain, susceptibility to optochin and bile solubility. S. pneumoniae isolates were serotyped by The National Centre for Streptococcus using the Quellung reaction. Penicillin MICs were determined by broth microdilution in accordance with Clinical and Laboratory Standards Institute recommendations.

RESULTS: From January 1, 2000 to June 30, 2007, 17 patients with HA pneumococcal BSI were identified, $0.7 \%$ of all HA BSI and $8.2 / 100,000$ admissions. There were no clusters identified nor a trend in frequency over time. Twelve of the $17(71 \%)$ patients were male and ages ranged from 3 months to 82 years. The most common risk factors were anemia, age $>65$ years, type II diabetes mellitus, malignancies, aboriginal descent and systemic corticosteroids. Fifteen of the 17 isolates were serotyped. The serotypes identified were $6 \mathrm{~A}, 6 \mathrm{~B}, 9 \mathrm{~V}, 11 \mathrm{~A}, 14,15 \mathrm{C}, 19 \mathrm{~F}, 23 \mathrm{~F}, 33 \mathrm{~F}$ and 38. Of these 10 serotypes, 2 were included in the PPV23 but not the PCV7, 5 were included in both and $3(18 \%)$ in neither. Of the 17 isolates, 14 (82\%) were susceptible and 3 intermediately resistant to penicillin.

CONCLUSIONS: Risk factors for HA pneumococcal BSI are similar to those for CA pneumococcal BSI. Ten different serotypes were identified of which three are not included in either PPV23 or PCV7. More than $80 \%$ of HA pneumococcal BSI were caused by penicillin-susceptible strains.

\section{P11}

\section{EVALUATION OF THE SPECTRAL RAPIDWN ${ }^{\mathrm{TM}}$ TEST FOR THE DETECTION OF WEST NILE VIRUS IGM ANTIBODIES IN PATIENTS' SERA SUSPECTED OF WEST NILE VIRUS INFECTION}

C Claessens $^{1 *}, \mathrm{~S}^{\text {Deraps }}{ }^{1}, \mathrm{~N}$ Shaikh ${ }^{2}$

${ }^{1}$ INSPQ/LSPQ, Sainte-Anne-de Bellevue, QC; ${ }^{2}$ Spectral Diagnostics Inc, Toronto, $\mathrm{ON}$

OBJECTIVE: To assess the clinical performance of Spectral RapidWN test for the qualitative detection of West Nile virus (WNV) IgM antibodies in patient's serum.

METHODS: Serum samples were tested with Spectral RapidWN test according to manufacturer's instructions. This test uses a solid-phase immunochromatography assay technology to qualitatively detect the presence of WNV IgM antibodies. The test is read for the presence of a coloured band 15 minutes after the addition of the diluted sample in the test device. A control band insures that all reagents were adequately added to the device. Samples with a minimal volume of $100 \mu \mathrm{l}$ were selected among sera received at our laboratory for WNV antibodies detection. Sera were stored below $-20^{\circ} \mathrm{C}$ after initial testing. Twenty five (25) sera positive by WNV IgM EIA (PanBio) from 18 patients with a confirmed infection to WNV were employed. Acute and convalescent sera from 6 patients and acute serum from 8 other patients were included. Infection of the patients was confirmed according to the standard algorithm requiring a positive plaque reduction neutralisation test on convalescent serum. Twenty (20) specimens negative for WNV specific IgM by EIA were also included in the study. 
RESULTS: For 14 patients, sera collected early in the course of disease were positive by EIA and RapidWN test for the presence of IgM. Four (4) out of 14 samples were only positive for WNV IgM, they did not contain specific WNV IgG as established by a negative result on WNV IgG EIA (PanBio). All convalescent samples were positive for IgM by EIA and the RapidWN test and positive for IgG by EIA. No invalid tests were observed. Concordance of results for positive and negative samples was $100 \%$.

CONCLUSION: Sensitivity of the test in this study was excellent compared to the EIA. The test is rapid and required minimal technical expertise. Reading of the positive and control bands was easy for almost every samples. Spectral RapidWN test has a distinct advantage over EIA in situations where a small number of samples need to be tested.

\section{P12}

DETECTION OF HUMAN HERPESVIRUS-6B IN

RESPIRATORY TRACT SAMPLES FROM CHILDREN WITH RESPIRATORY TRACT INFECTION

D Al-Nakhli ${ }^{*}$, K Luinstra ${ }^{2}$, A Petrich ${ }^{1,2}$, M Smieja ${ }^{1,2}$

${ }^{1}$ McMaster University, Hamilton, ON; ${ }^{2} \mathrm{St}$ Joseph's Healthcare,

Hamilton, ON

OBJECTIVES: Approximately 50\% of children undergoing nasopharyngeal sampling for diagnosis of respiratory tract infection do not have a respiratory virus demonstrated. We examined whether, and how frequently, HHV-6B was associated with acute upper respiratory tract infection in children less than 3 years of age.

METHODS: We sampled 98 children less than 3 years old, who had previously tested negative for respiratory viruses by a multiplex PCR capable of detecting 20 different viruses. These nasopharyngeal samples were chosen by age and month-stratified random sampling from clinical specimens submitted to the St. Joseph's Hospital Regional Virology Laboratory. Chart review was performed, with details of clinical presentation and course in hospital abstracted. HHV-6B specific PCR was performed.

RESULTS: In total, 8 of 98 (8.1\%) samples were positive by HHV-6B specific PCR. Amongst four children with data, 3 were male. Ages ranged from 8 to 24 months, and two had been born prematurely. Presentations included fever, cough, rhinorrhea, shortness of breath and anorexia. Physical findings consisted of tachypnea and tachycardia. Discharge diagnoses were: non-specific viral illness, bronchiolitis, pneumonia, upper respiratory tract infection and otitis media. Chest X-ray in 2 children was normal. Treatments included antibiotics (1), bronchodilators (3) and systemic steroids (1). Two were discharged on antibiotics and one on bronchodilator.

CONCLUSION: Detection of HHV-6B DNA, in young children with fever and acute respiratory illness but without evidence of any common respiratory virus, suggests that this virus may cause a small proportion of acute respiratory tract infection in these children. Timely diagnosis may help reduce unnecessary diagnostic procedures and treatments, but larger prospective studies are required.

\section{P13}

COMMUNITY OUTBREAK OF CYCLOSPORA CAYETANENSIS LINKED TO ORGANIC BASIL, BRITISH COLUMBIA, 2007 L Shah $^{1,2 *}$, L Macdougall ${ }^{2}$, A Ellis ${ }^{3}$, C Ong ${ }^{4}$, S Shyng ${ }^{5}$, L Leblanc ${ }^{6}$, Cyclospora Investigation Team Affiliations $\mathrm{P}^{7,8,9,10}$

${ }^{1}$ Canadian Field Epidemiology Program, Public Health Agency of Canada, Ottawa, ON; ${ }^{2}$ Epidemiology Services, British Columbia Centre for Disease Control, Vancouver, BC; ${ }^{3}$ Foodborne, Waterborne, Zoonotics Infections Division, Public Health Agency of Canada, Guelph, ON; ${ }^{4}$ Laboratory Services, British Columbia Centre for Disease Control; ${ }^{5}$ BC Food Protection, British Columbia Centre for Disease Control, Vancouver, BC; ${ }^{6}$ Canadian Food Inspection Agency, Ottawa, ON; ${ }^{7}$ Interior Health Authority, Kelowna; ${ }^{8}$ Fraser Health Authority, Surrey; ${ }^{9}$ Vancouver Island Health Authority; ${ }^{10}$ Vancouver Coastal Health Authority, Vancouver, BC

OBJECTIVE: Cyclospora outbreaks are difficult to investigate unless an event or limited food menu is involved. Food vehicles are often "stealth" ingredients such as garnishes which are not easily remembered. Additionally, implicated vehicles are often perishable and seldom available. Between May 1 and July 30, 2007, 23 cases of locally-acquired Cyclospora cayetanensis were reported in BC. In addition to routine epidemiologic investigation, early collaboration with the food regulators, collection of consumer card purchase records, product traceback for several suspect items and visits to the distributor were used collectively to identify the source of this outbreak.

METHODS: Cases were interviewed using a standardized hypothesisgenerating questionnaire. Questions on place of purchase, brands and cooking methods were asked for high frequency items. Detailed grocery store purchase histories were attained through cases' consumer cards. Population-based food consumption surveys were used as surrogate controls. Simultaneous traceback of several suspect vehicles identified common product consumption between cases. A visit to the distributor determined if product was marketed under other trade names.

RESULT: Seventeen cases were interviewed with a standardized questionnaire which did not identify a common event. Cilantro, basil, strawberries and garlic were consumed at higher frequency than controls. Only basil traced back to a common distributor; other items traced back to multiple intermediaries which did not obtain product from the same source. Overall, $82 \%$ of cases reported consuming organic basil during their incubation period. $53 \%$ of cases consumed basil exclusively from one organic distributor. Distributor records and consumer card data showed that organic basil was imported from an area previously linked to an outbreak. Importation continued later than usual, corresponding with temporal case distribution. No basil was available for testing.

CONCLUSION: Despite their rarity in North America, annual outbreaks of Cyclospora cayatenensis have occurred in BC since 1999. Most were not linked to events and often went unresolved. Basil has been previously implicated in outbreaks however this is the first identification of basil as the source of an outbreak in a community setting. The proactive use of combined investigative techniques was instrumental in implicating a single source.

\section{P14}

USING A LEAN SYSTEMS APPROACH (LSA) TO IMPROVE TIME TO REPORTING OF POSITIVE BLOOD CULTURES (BC)

D Roscoe*, A Whitley, H Garcha, S Namocatcat, J Reid, R Walker Vancouver General Hospital, Vancouver, BC

BACKGROUND: $\mathrm{BC}$ are one of the most critical specimens processed by microbiology laboratories. Turnaround time (TAT) from first identification of a positive BC to reporting final identification and susceptibility results is impacted by many factors. This study was done to determine if LSA could be used improve the time to reporting of positive BC.

METHODS: The steps of LSA were followed: 1) process selected, 2) problem statement and objectives developed, 3) team selected and trained, 4) baseline data obtained, and 5) changes implemented and evaluated during the run event. $\mathrm{BC}$ time to reporting was selected for the project according to the following criteria: importance of results, probability of success, visibility of impact, attitude of staff (can vs. can't), and strength of leadership. The project scope was all positive $\mathrm{BC}$ from receipt in the laboratory to final results reporting. An outside consultant assisted with LSA; 2 months were dedicated to planning and review and 1 month to implement suggestions and review results.

RESULTS: All steps in processing of positive $\mathrm{BC}$ were reviewed and value stream mapping was done. Data collected to determine current state revealed that only $20 \%$ of positive $\mathrm{BC}$ were finalized with identification and susceptibility within 24 hours of first flagging as positive. Controllable steps in $\mathrm{BC}$ processing identified to have an impact on this figure were: time to accession $\mathrm{BC}$, time to load $\mathrm{BC}$ into automated equipment, time to investigate $\mathrm{BC}$ signaled as positive, review of culture for early growth to begin investigations, time to receive, review and release results of identification and susceptibility tests, the number of technologists working on $\mathrm{BC}$ at critical points, and the order of work flow on the BC bench.

CONCLUSIONS: Applications of LSA have not been widely reported from Microbiology Laboratories. Preliminary results from this project have 
implicated key steps to improve BC processing and final data and impact on BC TAT will be presented. LSA has already demonstrated benefits to other processes within our microbiology laboratory including cleaning and de-cluttering, re-organizing and standardizing bench locations, and reorganizing bench set-up. As well, laboratory staff have been enthusiastic and energized by the process.

\section{P15}

\section{A NOVEL APPROACH FOR DEVELOPING AN INTERNAL POSITIVE CONTROL USING CHIMERIC DNA FOR MOLECULAR TESTS}

M-K Lee ${ }^{1 *}$, D Eisler ${ }^{1}$, D Jorgensen ${ }^{1}$, Q Wong1 ${ }^{1}, M$ Morshed ${ }^{1,2}$ ${ }^{1} \mathrm{BC}$ Centre for Disease Control, Vancouver, BC; ${ }^{2}$ University of British Columbia, Vancouver, BC

OBJECTIVE: Internal positive control (IPC)s are important in the quality control of molecular testing, being used to detect PCR inhibitors and to rule out false negative results. However, the scarcity of commercial IPCs and the labour involved in developing in-house IPCs for each assay prompted us to develop a single chimeric IPC employable on different platforms and with various targets.

METHODS: Design: A DNA construct was designed, from 5' to 3', of tandemly-arranged forward primer sequences of multiple targets, followed by a probe binding region and the reverse complement of the targets' respective reverse primers, also in tandem. Composition: The IPC testing templates were generated on a Stratagene Robocycler using primers designed to amplify the IPC construct. The amplified template was cloned into $\mathrm{pCR}{ }^{\circledR}$ 4-TOPO and plasmid preparations were prepared from transformed E. coli Mach1T1 cells using Purelink Plasmid Preparation kits (Invitrogen). DNA extraction and clean up were performed using QIAgen Extraction kits and real time PCR was performed on an Applied Biosystems 7500 Real Time instrument. The IPC can be added directly to PCR reactions or cloned into $E$. coli to mimic a real organism.

RESULTS: Three targets tested with this system confirmed that the IPC is easily distinguished from target PCR products using gel electrophoresis, due to differences in amplicon sizes and it can be detected on a real-time platform with corresponding fluorescent probe.

CONCLUSION: The use of a chimeric internal positive control in PCR provides an inexpensive, customizable approach to enhancing PCR quality control, which has the added benefit of being easy to develop, maintain and troubleshoot in cases of carryover contamination.

\section{P16}

EVALUATION OF EXTRACTION METHODS FOR THE DETECTION OF NEUROTROPIC VIRUSES IN CEREBROSPINAL FLUID

HZ Johnson ${ }^{1 *}$, V Khurana ${ }^{1}$, G Appleyard ${ }^{1}$, J Fox ${ }^{1,2}$

1Provincial Laboratory for Public Health (Microbiology), Calgary, AB; ${ }^{2}$ Microbiology \& Infectious Diseases, University of Calgary, Calgary, AB

OBJECTIVE: Testing cerebrospinal fluid (CSF) for neurotropic viruses is critical in aiding the diagnosis and management of encephalitis or meningitis. An efficient and adaptable extraction method and testing platform is desirable due to wide fluctuations in annual specimen submissions. The objective of the study was to evaluate two commercial extraction methods for contribution to assay sensitivity (A: detection limit) and assay performance (B: agreement).

METHODS: A) Ten-fold dilution series of cultured HSV, VZV, enterovirus, and WNV were spiked into a pool of negative CSF. These were then extracted by both the QIAamp ${ }^{\circledR}$ Viral RNA (Qiagen) and easyMAG® (bioMérieux) kits and tested in triplicate by appropriate virusspecific assays (artus-Biotech assays from Qiagen, in-house developed PCR and NASBA assays) to determine detection limits. B) Known-positive CSF specimens (VZV $\mathrm{n}=16$, HSV $\mathrm{n}=22$ ) originally extracted in 2005-2007, by QIAamp Viral RNA kit, were re-extracted by the easyMAG method and were re-tested using either the artus VZV LC assay or HSV in-house LC assay. Results were compared for agreement in crossing points $(\mathrm{CP})$.
RESULTS: A) Performance of the artus and in-house assays for detection of HSV were nearly identical for each of the extraction methods (mean diff $<0.33 \mathrm{CP}$ ). Detection of VZV by artus had consistently lower CP for specimens extracted by easyMAG (mean diff $1.3 \mathrm{CP}$ ). Enterovirus was detected by artus with lower CP using Qiagen extraction (mean diff 0.84 $\mathrm{CP}$ ); however WNV was detected by artus with lower CP using easyMAG extraction (mean difference 1.24 CP). Detection limit for enterovirus and WNV by NASBA was 10 to 100 -fold lower by easyMAG extraction. B) Analysis of paired HSV positive specimens for the two extraction methods showed strong agreement $\left(\mathrm{r}^{2}=0.97\right.$, mean diff $\left.0.5 \mathrm{CP}\right)$, whereas for VZV positive specimens the correlation was not as strong $\left(\mathrm{r}^{2}=0.70\right.$, mean diff $0.62 \mathrm{CP}$ )

CONCLUSION: The two extraction methods perform comparably. When paired with NASBA, the easyMAG method supported a lower detection limit for WNV, while Qiagen extractions, when paired with the artus enterovirus detection kit supported a wider dynamic range. The easyMAG method consistently supported lower CP for VZV detection. Results for HSV were nearly indistinguishable between extraction methods.

\section{Poster Presentations Friday, February 29 Grand Ballroom (Exhibit Hall)}

\section{P17}

\section{AN ALBERTAN VISA? A CLINICAL AND MICROBIOLOGIC STORY OF A VANCOMYCIN INTERMEDIATE STAPHYLOCOCCUS AUREUS}

RA Harrison $^{1 *}$, J Ahmed-Bentley ${ }^{2}$, J Fuller ${ }^{3}$, LM Saxinger ${ }^{1}$

${ }^{1}$ University of Alberta Hospital; ${ }^{2}$ Dynacare Kasper Medical Laboratories; ${ }^{3}$ Provincial Laboratory Alberta, Edmonton, AB OBJECTIVE: To document evidence of isolation of vancomycin intermediate Staphylococcus aureus (VISA) from a clinical patient specimen despite very minimal exposure to vancomycin therapy, and to describe the patient's course and successful therapy with linezolid.

METHODS: The patient's hospital charts were reviewed. Microbiologic methods involved identification of two strains of MRSA from a clinical specimen according to standard laboratory procedures by Vitek ${ }^{\circledR}$ automated susceptibility testing, commercial vancomycin and oxacillin screen plates, and cefoxitin disc diffusion. The vancomycin minimum inhibitory concentrations (MIC) were tested by Vitek $\AA$ and E-test ${ }^{\circledR}$ Glycopeptide susceptibility results were confirmed by repeat in-house testing and referral to a reference laboratory. PCR for van A, B, C1, C2/C3, and D genes was used to detect genetic evidence of vancomycin resistance.

RESULTS: A 23-year-old male had surgical resection of an orbit chondrosarcoma, complicated by CMRSA 2 infection (identified by BD Phoenix $^{\mathrm{TM}}$ automated system postoperative day $27=$ Day 1$)$. The patient only received a total of 7 days of vancomycin therapy (days 5-11, and day 51) prior to isolation of a VISA strain of MRSA on day 118. At that point, osteomyelitis of the orbital bone was diagnosed and VISA, Streptococcus pyogenes, anginosus group Streptococcus sp., Candida albicans, and Corynebacteria sp. were all isolated from a swab of the infected area. The MIC of vancomycin for the VISA strain was $3 \mathrm{ug} / \mathrm{ml}$ by E-test, and van genes were not detected by PCR. Treatment with surgical debridement and 6 weeks of oral linezolid was successful, with no evidence of infection recurrence.

CONCLUSION: We report a Canadian VISA infection that appeared to evolve after very minimal and remote exposure to vancomycin. Previous reports document prolonged vancomycin exposure as the main risk factor for VISA infection, but minimal vancomycin exposure may promote emergence of VISA. Reliance on automated systems alone may not be adequate for diagnosis of VISA strains. Standardized laboratory approaches and definitions will be key in surveillance, and with clinical context, should help elucidate predictable and preventable risk factors for VISA infection. 
P18

DAPTOMYCIN (DAP) ACTIVITY TESTED AGAINST GRAMPOSITIVE (GP) COCCI ISOLATED IN CANADIAN MEDICAL CENTERS (2004-2007)

DI Roscoe ${ }^{1 *}$, DJ Hoban ${ }^{1,2}$, MA Desjardins ${ }^{1,3}$, KR Forward ${ }^{1,4}$, R Rennie ${ }^{1,5}$, H Sader ${ }^{1,6}$

${ }^{1}$ Vancouver General Hospital, Vancouver, BC; ${ }^{2}$ Health Sciences Centre, Winnipeg, $\mathrm{MB} ;{ }^{3}$ Ottawa Hospital, General Campus, Ottawa, ON; ${ }^{4}$ Queen Elizabeth II Health Sciences Centre, Halifax, NS; ${ }^{5}$ University of Alberta Hospital, Edmonton, AB; ${ }^{6} \mathrm{JMI}$

Laboratories, North Liberty, IA, United States

BACKGROUND: DAP is a cyclic lipopeptide with broad spectrum and potent antimicrobial activity against GP pathogens. DAP is approved by Health Canada for the treatment of complicated skin and skin structure infections (cSSSI) and S. aureus bacteremia (B), including right-sided endocarditis.

METHODS: 2,737 bacterial isolates were consecutively collected in 2004-2007 in 5 Canadian hospitals from various types of infections, mainly B and cSSSI. Isolates were tested for susceptibility (S) to DAP (50 $\mathrm{mg} / \mathrm{L}$ calcium) and comparators using the CLSI broth microdilution methods against.

Results: All isolates were $\mathrm{S}$ to DAP - see table. DAP was the most potent (lowest $\mathrm{MIC}_{90}$ ) among selected antimicrobials commonly used to treat GP infections, including vancomycin (VAN), teicoplanin, linezolid, and $\beta$-lactam and fluoroquinolone compounds.

Cumulative \% inhibited at MIC $\mathrm{mg} / \mathrm{L}$ ):

\begin{tabular}{lcccccc} 
Organism (no tested) & $\mathbf{5 0 . 1 2}$ & $\mathbf{0 . 2 5}$ & $\mathbf{0 . 5}$ & $\mathbf{1}$ & $\mathbf{2}$ & $\mathbf{4}$ \\
\hline Methicillin (MET)-S S. aureus (SA; 747) & 4.7 & 80.9 & 99.9 & 100.0 & - & - \\
MET-resistant (R) SA (MRSA; 368) & 1.6 & 75.4 & 98.6 & 100.0 & - & - \\
Coagulase-neg. staph. (CoNS; 589) & 7.5 & 58.2 & 97.3 & 100.0 & - & - \\
E. faecalis (432) & 0.7 & 4.6 & 54.2 & 94.9 & 99.1 & 100.0 \\
VAN-S E. faecium (106) & 0.0 & 1.9 & 4.7 & 28.3 & 79.3 & 100.0 \\
VAN-R E. faecium (18) & 0.0 & 0.0 & 0.0 & 44.4 & 100.0 & - \\
$\beta$-haemolytic streptococci (246) & 78.1 & 97.6 & 100.0 & - & - & - \\
Viridans group streptococci (209) & 35.4 & 65.1 & 92.8 & 100.0 & - & - \\
\hline
\end{tabular}

33.0 and $70.1 \%$ of SA and CoNS were MET-R, respectively, and DAP demonstrated excellent activity against both MET-S and MET-R staphylococci $\left(\mathrm{MIC}_{50 / 90}, 0.25 / 0.5 \mathrm{mg} / \mathrm{L}\right)$. VAN resistance (VRE) was detected among $12.9 \%$ of $E$. faecium, but DAP remained highly active against these strains $\left(\mathrm{MIC}_{90}, 2 \mathrm{mg} / \mathrm{L}\right)$.

CONCLUSIONS: Resistance to MET or VAN did not adversely influence DAP activity against staphylococci or enterococci. DAP demonstrated significant potency and spectrum against contemporary GP bacteria isolated in Canada, including MRSA and VRE.

\section{P19}

COMPARISON OF OXOID MIC EVALUATOR (MICE ${ }^{\mathrm{TM}}$ ) DEVICE WITH BROTH MICRO-DILUTION (BMD) AND ETEST ${ }^{\circledR}$ DEVICE FROM AB BIODISK FOR ANTIMICROBIAL SUSCEPTIBILITY TESTING OF ENTEROBACTERIACEAE

R Rennie*, L Turnbull, C Brosnikoff

University of Alberta Hospital, Edmonton, AB

OBJECTIVE: The current study was designed to evaluate a new product utilizing an agar based gradient endpoint system $\left(\mathrm{MICE}^{\mathrm{TM}}\right)$ produced by Oxoid (Thermo Fisher Scientific) as an alternative to automated systems for accurate MIC test results of Enterobacteriaceae that can be utilized effectively in critical care. The MICE ${ }^{\mathrm{TM}}$ product is not available for sale or use in the United States.

METHODS: 250 recent clinical strains of Enterobacteriaceae, including 16 genera and over 30 species were tested. Initially, seven evaluable antimicrobials were tested: ciprofloxacin, amoxicillin, amoxicillin clavulanate, ampicillin, levofloxacin; and cefotaxime and gentamicin (low and high concentrations). The strains were inoculated into BMD trays (CLSI M7-A7) and onto Mueller Hinton agar. The MIC strips were added to the plates according to each manufacturer's instructions and were incubated for $20-24 \mathrm{hr}$ at $35^{\circ} \mathrm{C}$. Inhibition was read where the elliptical zone intersected with the strips. ATCC E. coli 25922 P. aeruginosa 27853, S. aureus 29213 and E. faecalis 29212 were the quality control strains.

RESULTS: Quality control stains were within CLSI published ranges. For the clinical strains, 2250 agent-organism MIC results were available for analysis. Over $98 \%$ of results were within 2 doubling dilutions comparing $\mathrm{BMD}$ to either MICE ${ }^{\mathrm{TM}}$ or $\mathrm{AB}$ Biodisk's Etest ${ }^{\circledR}$ device. Compared to BMD there were 8 Very Major errors (0.4\%), 1 Major error (0.04\%) and 7 minor errors $(0.3 \%)$ observed with both devices. Six of the $8 \mathrm{VM}$ errors occurred with amoxicillin and ampicillin, and with Proteus species.

CONCLUSIONS: MICE $^{\mathrm{TM}}$ device results for clinical strains of Enterobacteriaceae were comparable to the gold-standard CLSI BMD observations and to Etest ${ }^{\circledR}$ device results for seven evaluable antimicrobial agents. Additional antimicrobials are undergoing evaluation. The gradient endpoint MIC device provides accurate susceptibility test results for Enterobacteriaceae.

\section{P20}

ANTIMICROBIAL ACTIVITY AND SAFETY OF COENCAPSULATED GENTAMICIN AND GALLIUM INTO LIPOSOMAL FORMULATION

M Halwani ${ }^{1}$, B Yebio ${ }^{1}$, M Alipour ${ }^{1}$, ZE Suntres ${ }^{2}$, A Kumar ${ }^{1}$, A Omri ${ }^{1}$ ${ }^{1}$ Laurentian University, Sudbury; ${ }^{2}$ Lakehead University, Thunder Bay, ON

INTRODUCTION: Decline in the health status of individuals with cystic fibrosis (CF) is known to be associated with infections by opportunistic gram-negative bacteria such as $P$. aeruginosa (PA) and B. cepacia (BC). These pathogens have shown intrinsic resistance through different mechanisms to aminoglycosides (i.e. Gentamicin [GN]). Delivery of GN as a liposomal formulation has increased susceptibility of resistant bacteria to the antibiotic. To further improve the antibiotic efficacy of this liposomal formulation, we have incorporated gallium [GAL] in the liposomal-GN formulation (LGG). GAL is known to have inhibitory effects on several gram positive and negative bacteria by disrupting the iron metabolism of the microorganisms.

METHODS: Dehydration-rehydration method was used to prepare LGG. Dynamic light scattering was applied to measure vesicle size. Atomic absorption spectroscopy and microbiological assay were performed to determine the encapsulation efficiency and stability of GAL and GN within the liposomal formulations, respectively. Clinical strain isolates from CF patients' samples were used to determine minimal inhibitory and bactericidal concentrations (MIC and MBC) of these formulations. The viability of human lung epithelial A549 cells was measured by trypan blue assay after $24 \mathrm{~h}$ exposure to free and liposomal-GAL in order to assess the toxicity of GAL.

RESULTS: The size of the LGG was $337.26 \pm 34.88 \mathrm{~nm}$ with an encapsulation efficiency of $4.31 \pm 0.26 \%$ for GN. Retention of GN in liposomes was $89.89 \%$ and $87.47 \%$ in $\mathrm{PBS}$ at $4^{\circ} \mathrm{C}$ and in $\mathrm{BAL}$ at $37^{\circ} \mathrm{C}$ in a period of $48 \mathrm{~h}$, respectively. However, the retention was significantly reduced to $51.85 \%$ when the LGG was incubated in plasma at $37^{\circ} \mathrm{C}$ for 48 hours. The $\mathrm{MIC}$ and $\mathrm{MBC}$ values indicated that $\mathrm{GN}$ was more effective against highly resistant strains of $\mathrm{PA}$ and $\mathrm{BC}$ when it was delivered as a liposomal-GAL formulation. Cell viability of human A549 lung cells incubated with LGG was higher compared to free GAL, suggesting that encapsulation of GAL within liposomes lowers the toxicity of GAL.

CONCLUSION: Incorporation of GAL into liposomes containing GN could be utilized as a new strategy to enhance the efficacy of existing antibiotics against resistant pathogens. 


\section{P21}

ANTIMICROBIAL RESISTANCE IN PATHOGENS ISOLATED FROM CANADIAN HOSPITAL OUTPATIENT CLINICS (C), EMERGENCY ROOMS (ER), MEDICAL/SURGICAL WARDS (W) AND INTENSIVE CARE UNITS (ICU): RESULTS OF THE CANWARD 2007 STUDY

M Decorby ${ }^{1}{ }^{*}$, B Weshnoweski ${ }^{2}$, R Vashisht ${ }^{1}$, N Laing1, F Tailor ${ }^{1}$, N Chiu ${ }^{1}$, KA Nichol ${ }^{2}$, P Baudry ${ }^{1}$, A Wierzbowski ${ }^{1}$, M McCracken $^{3}$, M Mulvey ${ }^{1,3}$, P Lagace-Wiens ${ }^{1}$, A Walkty ${ }^{1}$, DJ Hoban ${ }^{1,2}$, GG Zhanel ${ }^{1}$

${ }^{1}$ University of Manitoba, Winnipeg, MB; ${ }^{2} \mathrm{Health}$ Sciences Centre/Diagnostic Services of Manitoba, Winnipeg, MB; ${ }^{3}$ National Microbiology Laboratory, Winnipeg, MB

BACKGROUND: Antimicrobial resistance is a worldwide concern. The CANWARD study assessed the types of pathogens causing infections in patients affiliated with Canadian hospitals, as well as the prevalence of antimicrobial resistance in these isolates.

METHODS: In a prospective, population based study, 12 sentinel centres across Canada submitted pathogens causing infections from patients attending outpatient clinics (C), emergency rooms (ER), medical and surgical wards (W) and intensive care units (ICU). Susceptibility testing was performed using CLSI broth microdilution methods.

RESULTS: 5851 isolates have been collected to date: $34.6 \%$ from blood, $33.2 \%, 21.9 \%$, and $10.3 \%$ from respiratory, urine and wound/IV site specimens, respectively. Patient demographics were $53.6 \% / 46.4 \%$ male/female, $12.2 \%$ from $\leq 17$ yrs, $46.2 \%$ from $18-64$ yrs and $41.6 \%$ from $\geq 65$ yrs. Isolates were obtained from patients on W 39.6\%, ER 25.3\%, C 20.1\% and ICU $15.0 \%$. The most common pathogens were: E. coli $22.5 \%$, S. aureus (MSSA) $14.0 \%$, P. aeruginosa 9.2\%, S. pneumoniae 8.8\%, Enterococcus spp. $6.1 \%, K$. pneumoniae $5.4 \%$ and MRSA $5.0 \%$. The overall prevalence of MRSA, VRE, and ESBL-producing E.coli was: (295/1113) 26.5\%, (10/355) 6.1\%, and (34/1227) 2.8\%, respectively. Resistance rates (RR) for E. coli were: $0 \%$ meropenem (MER), $0 \%$ tigecycline (TGC), $1.6 \%$ piperacillin/tazobactam (PTZ), 6.9\% ceftriaxone (CTR), 10.6\% gentamicin (GEN), 24.6\% trimethoprim/sulfamethoxazole (SXT) and $24.6 \%$ ciprofloxacin (CIP). RR for P. aeruginosa were: $8.3 \%$ PTZ, 8.9\% MER, $13.2 \%$ cefepime, $24.1 \%$ GEN and $23.9 \%$ CIP. RR for MRSA were: $0 \%$ vancomycin (VAN), linezolid (LZD), TGC and daptomycin (DAP), 15.0\% SXT, $62.2 \%$ clindamycin, $90.2 \%$ clarithromycin and $88.2 \%$ for CIP. CONCLUSIONS: The most common pathogens isolated in patients affiliated with Canadian hospitals were E. coli, S. aureus (MSSA), P. aeruginosa, Enterococcus spp., S. pneumoniae and MRSA. RR for E. coli and P. aeruginosa were lowest with MER and PTZ. For MRSA, no resistance occurred with VAN, LZD and TGC.

\section{P22}

DEVELOPMENT OF NOVEL SYBR GREEN REAL-TIME PCR ASSAYS FOR THE DETECTION OF MYCOPLASMA HOMINIS AND UREAPLASMA UREALYTICUM

A Lum $^{1 *}$, E Thomas ${ }^{2}$, J Brunstein ${ }^{1,2}$

${ }^{1}$ Centre for Translational and Applied Genomics, PHSA Labs, Vancouver, BC; ${ }^{2}$ Department of Pathology and Laboratory Medicine, Children's and Women's Health Centre of BC, PHSA Labs, Vancouver, BC

OBJECTIVE: Mycoplasma hominis and Ureaplasma urealyticum are pathogens associated with prenatal intrauterine infection leading to premature birth and perinatal morbidity and mortality. The prevalence of M. hominis and Ureaplasma spp. are between 21 to $53 \%$ and 40 to $80 \%$, respectively, on the cervical or vaginal mucosa of healthy females with a vertical transmission rate of 18 to $88 \%$ (inversely related to gestational age). Colonization of the respiratory mucosa in neonates has been associated with neonatal pneumonia and increased risk of developing chronic lung disease or bronchopulmonary dysplasia, as well as pneumonitis in immunocompromised hosts. As isolation using conventional culture methods is difficult and time-consuming, we attempted to develop two Sybr Green real-time PCR assays for the detection of M. hominis and all 14 serovars of $U$. urealyticum.
METHODS: Two separate SYBR Green based PCR assays were developed for the detection of M. hominis by the gyrB gene and $U$. urealyticum by the ureC gene. Positively identified isolates were used for development and validation of these assays. Samples were extracted by the Qiagen Biorobot M48 automated DNA/RNA extractor, or manually using the Qiagen DNA mini kit and run on the Cepheid SmartCycler in $25 \mu \mathrm{L}$ reactions.

RESULTS: Both M. hominis and U. urealyticum PCR assays were successfully developed on the SmartCycler with a run time of approximately 90 minutes per assay. Although the two species are related, there was no apparent cross reactivity between the M. hominis assay and $U$. urealyticum or between the $U$. urealyticum assay and $M$. hominis.

CONCLUSIONS: The development of two molecular assays for $M$. hominis and $U$. urealyticum drastically improves turnaround time from several days to only a few hours. A rapid diagnosis, therefore treatment, of neonates exhibiting respiratory distress may prevent dissemination into more serious infections such as bacteremia or meningitis.

\section{P23}

PHENOTYPIC AND GENOTYPIC ANALYSIS OF BORDETELLA PERTUSSIS STRAINS ISOLATED IN ONTARIO, CANADA M Sill1*, I Martin ${ }^{1}$, J Stoltz' ${ }^{1}$, F Jamieson ${ }^{2}$, S Brown ${ }^{2}$, P Tang ${ }^{2}$, A Chagla ${ }^{2}$, A Majury ${ }^{2}$, J Jessop ${ }^{2}$, R Tsang ${ }^{1}$

${ }^{1}$ National Microbiology Laboratory, Public Health Agency of Canada, Winnipeg, MB; ${ }^{2}$ Ontario Public Health Laboratory, Ministry of Health and Long Term Care, Toronto, ON

OBJECTIVE: Increase in pertussis disease activity, especially in adolescents and adults, has been reported in countries with high vaccination rates, including Canada. In order to understand the characteristics of these disease-causing strains, we characterized B. pertussis isolates from Ontario by serotyping, pulsed-field gel electrophoresis (PFGE), and DNA sequencing of pertussis virulence antigen genes.

METHODS: 521 strains of B. pertussis isolated from 1998-2006 were characterized by serotype, PFGE using XbaI and SpeI restriction enzymes, and partial sequencing of the following virulence genes that are components of the acellular pertussis vaccine: pertussis toxin ( $p t x S 1)$, pertactin $\left(p r n_{1}\right)$, filamentous hemagglutinin (fhaB) and fimbriae (fim3).

RESULTS: Most of the 521 strains were isolated in 2004-2005 (294 isolates or 56\%). Age information was available for 244 cases, and ranged from 19 days to 92 years, with a mean age of 16.7 years. Of the 521 isolates, 517 were serotype Fim3, one was Fim2, two expressed both Fim2 and 3, and one isolate was not typeable. PFGE analysis identified 69 different DNA fingerprint profiles, with an overall similarity of $80 \%$. The 7 most common DNA profiles shared $88.5 \%$ similarity and accounted for 410 isolates $(78.7 \%)$. Three profiles, consisting of 332 isolates $(63.7 \%)$, were identical to PFGE group $I_{\beta}$ profiles found in Sweden. PFGE group $I_{\beta}$ is a common group found among $B$. pertussis clinical isolates in Europe. The predominant genotype in the Ontario isolates was ptxS1A, prn21, fhaB1, fim 3B (437 isolates or $83.9 \%$ ).

CONCLUSION: Recent isolates of B. pertussis from Ontario appeared to be similar to those currently circulating in Europe. A combination of both genetic sequence and PFGE analysis showed that most isolates collected in 2004-2005 appeared to be highly related. Variations in fimbriae, pertactin, and pertussis toxin genes were detected. It is not currently known if genetic polymorphisms in B. pertussis contribute towards disease evolution pattern. However, continued surveillance involving characterization of strains will help to identify genetic events that may be associated with vaccine escape mechanism and to provide information on the choice of vaccine strains, should it become necessary to match characteristics of vaccine strains with those found circulating in the population.

\section{P24}

\section{ACCURACY OF ESBL DETECTION BY THE BD PHOENIX ANALYSER}

L Borycheski*, A Sarabia

The Credit Valley Hospital, Mississauga, ON

OBJECTIVE: The purpose of the study was to evaluate the accuracy of the BD Phoenix ${ }^{\circledR}$ system in the detection of ESBL-producing organisms 
using the CLSI-recommended confirmatory disk tests and a Reference Laboratory result as the reference standards.

METHODS: BD Phoenix panel NMIC/ID-24 is a combination identification and breakpoint susceptibility panel. The isolates flagged by the Phoenix as ESBL-producing organisms were further analysed using the confirmatory method of MASTDISCS ID*. This system is a double disc method consisting of cefpodoxime, ceftazidime and cefotaxime, each with and without clavulanic acid. A positive result from any or all of the pairs of MAST ID ESBL Detection Discs confirms ESBL production. Isolates that flagged as ESBL-positive by the Phoenix which did not confirm by the MAST Discs were sent to the Public Health Laboratory for ESBL testing. RESULTS: Between March 2005 and August 2006, 90 isolates were identified as ESBL positive by the Phoenix ${ }^{\circledR}$. Eighty isolates were Escherichia coli, 8 were Klebsiella pneumoniae, and 2 were Klebsiella oxytoca. Forty-eight (53\%) were confirmed as ESBL positive by MAST testing. Forty-two (47\%) did not confirm by MAST. Thirty-nine of the 42 isolates that did not confirm by MAST were sent to PHL for ESBL testing. None of these were confirmed to be Class A ESBL. Ten of 39 were reported as AmpCtype ESBL. The Phoenix erroneously reported 42 of the 90 flagged isolates as $\operatorname{ESBL}(47 \%)$.

CONCLUSION: Despite previously published reports of accuracies of $89 \%$ for ESBL detection by the BD Phoenix system, in our hands, the system erroneously reported organisms as ESBL producers $47 \%$ of the time. After reviewing the collected data, an algorithm was developed to determine if the organism was an ESBL producer without having to use alternate methodology such as the MAST Disc or referring the isolates to a reference laboratory. The algorithm will lessen the workload as well as decrease the amount of time when reporting an organism as an ESBL producer. The algorithm will be periodically audited by using the MAST Disc to ensure that it reliably detects ESBL producers.

\section{P25}

EVALUATION OF CHROMID VRE AGAR FOR ISOLATION AND DIFFERENTIATION OF VANCOMYCIN-RESISTANT ENTEROCOCCUS

R Woolven*, F Papadopoulos, L Sexsmith, H Lundy Quinte Health Care, Belleville, ON

OBJECTIVE: Rapid turnaround time for screening of specimens is essential in preventing the transmission of Vancomycin-Resistant Enterococcus (VRE). Chromogenic agar media with the capability to both recover and identify vancomycin-resistant Enterococcus faecium and Enterococcus faecalis have the potential to significantly reduce turnaround time in the detection of VRE colonized patients. Cost savings due to reduced work-up of isolates should also result. The objective of this study was to evaluate chromID VRE Agar in the isolation and differentiation of VRE and compare its performance to Bile Esculin Azide Agar supplemented with vancomycin (BEAv).

METHODS: Four hundred rectal swabs collected for admission screening purposes or during point prevalence screens were inoculated directly onto chromID VRE and BEAv. Plates were examined at 24 and 48 hours for growth characteristic of enterococci. Suspect colonies were identified to species and vancomycin MIC determined.

RESULTS: A total of sixteen VRE (six vanA and ten vanB strains of Enterococcus faecium) were isolated on at least one of the two media. The chromID VRE recovered fifteen VRE including five that were not detected by the BEAv. Eleven VRE were recovered by BEAv including one not detected by chromID VRE. Eighteen suspect isolates from BEAv and eight from chromID VRE requiring full work-up were subsequently identified as enterococci not VRE.

CONCLUSION: Improved recovery rates were seen with chromID VRE as compared to BEAv. In addition, there were fewer false positives with chromID VRE which resulted in the use of fewer resources.

\section{P26}

EVALUATION OF RAPID ASSAYS TO DETECT GIARDIA AND CRYPTOSPORIDIUM

\section{Alawi*, M Smieja, R McQueen}

McMaster Hospital, Hamilton, ON

INTRODUCTION: Giardiasis and cryptosporidiosis are two common causes of protozoan waterborne diarrheal outbreaks in humans. Giardia lamblia and Cryptosporidium parvum can cause severe symptoms especially in immune-compromised persons. There are no characteristic laboratory features of Cryptosporidium infection other than identification of the organisms using modified acid-fast stains that are labour intensive and time consuming, and require well-trained technologists.

OBJECTIVE: Comparison of two non-enzymatic rapid immunoassays with standard microscopic methodsto diagnose Giardia and Cryptosporidium.

METHODS: 111 stool samples were collected in sodium acetate- acetic acid-formaldehyde (SAF) which contained a variety of parasites including Giardia and Cryptosporidium from parasitological laboratories from St. Joseph's Hospital (that includes all samples from the Hamilton region), MDS and Central Public Health Laboratory both in Toronto, between November, 2006 and January, 2007, and stored at $-2^{\circ} \mathrm{C}$. 93 samples out of 111 samples were randomly selected and tested using Prospect microwell EIA. 40 out of 111 samples were blindly tested by Xpect Giardia/Cryptosporidium.

RESULT: The sensitivities with Xpect for Giardia and Cryptosporidium were $66.6 \%$ and $100 \%$ respectively. The specificities were $100 \%$ for both. The Xpect assay was easy to do and interpret.The sensitivities with Prospect for Giardia and Cryptosporidium were $82.8 \%$ and $85.7 \%$. The specificities were $68 \%$ for both.

CONCLUSION: The Xpect looked promising especially in that it had high sensitivity and specificity for Cryptosporidium but the assay requires further investigation, with a larger number of stored and fresh specimens. Based on these preliminary results, it has potential utility for outbreaks. However, neither the Xpect assay for Giardia, nor the Prospect assay for either Cryptosporidium or Giardia, was as sensitive as light microscopy. The Prospect assay gave sub-optimal results for both infections.

\section{P27}

VALIDATION OF STREP B CARROT BROTH USING A PROSPECTIVE DOUBLE SWAB COMPARISON METHOD M Miller $^{1 *}$, L Tomalty ${ }^{1,2}$, R Liao ${ }^{1,2}$, D Zoutman ${ }^{1,2}$

${ }^{1}$ Kingston General Hospital, Kingston, ON; ${ }^{2}$ Queens University, Kingston, $\mathrm{ON}$

OBJECTIVE: Screening for Group B Streptococci (GBS) in prenatal women at 35-37 weeks continues to be a workload and procedural issue for clinical microbiology laboratories. We set out to conduct a prospective comparative study for the use of Strep B Carrot Broth (Hardy Diagnostics) versus GBS Broth (PML Microbiologicals) for GBS screening.

METHODS: 200 combined vaginal/rectal swabs were collected at 35-37 weeks gestation. Collection was performed in parallel using a double headed swab. Swab \# 1 was inoculated into Strep B Carrot Broth. Swab \#2 was inoculated into GBS Broth. The GBS Broth was incubated for 24 hours and then plated to CNA. After overnight incubation any Carrot Broth which changed from colourless to orange was screened directly with latex agglutination testing (Prolex Diagnostics) using Group B latex. After the initial overnight incubation all Carrot Broths were subcultured to CNA to confirm the agglutination testing results and to capture any potential false negatives such as non hemolytic GBS that fail to produce the caratenoid pigment that will turn the Carrot Broth orange.

RESULTS: $55 / 200$ (28\%) of the swabs were positive for GBS, 55/200 (28\%) were positive with Carrot Broth, 49/200 (25\%) were positive using the conventional GBS Broth method. The Carrot Broth captured 6/200 (3\%) positives which were missed using the standard culture method. All Carrot Broths that turned orange confirmed as being positive for GBS. $47 / 200(24 \%)$ of our samples were direct latex agglutination positive for Group B from the Carrot Broth; providing results within 24 hours. The average TAT for the Carrot Broth was 24-48 hr compared to our standard 
culture method which was requires 48-96 hr. Carrot Broths subcultured to CNA for confirmation and exclusion of nonhemolytic GBS were also macroscopically easier to read due to inhibition of commensal bacteria. CONCLUSION: Testing with Carrot Broth will decrease workload since $24 \%$ of samples were completed on day 1 without subculture. A continued limitation of GBS testing remains the requirement for subculture to solid agar media. Strep B Carrot Broth does not detect nonhemolytic GBS and in this study only 48 of 55 (87\%) positive GBS Strep B Carrot Broths changed in colour to orange indicating positivity. This product shows increased sensitivity, specificity and a marked decrease in TAT compared to conventional testing.

\section{P28}

CLINICAL EVALUATION OF A PRESERVATIVE IMPREGNATED SPONGE FOR TRANSPORT OF URINE SAMPLES FOR MICROBIOLOGICAL CULTURE

L Turnbull*, C Brosnikoff, R Rennie University of Alberta Hospitals Medical Microbiology Department, Edmonton, AB

OBJECTIVE: Evaluation of the clinical ability of a preservative impregnated sponge to preserve the quantity and quality of bacterial pathogens in urine during transport.

METHODS: 935 urines collected for culture were poured over a routine culture paddle (Starplex Dip N' Count). The preservative sponge (Uriswab, Copan Italia) was then dipped into the remaining urine to absorb the sample (approx. 5s). The culture paddle was incubated 18 to $24 \mathrm{~h}$ and read according to standard quantitation protocols. Urine from the sponge was cultured by squeezing the tube to express the urine; and with a $0.001 \mathrm{ml}$ calibrated loop, the expressed urine was plated onto blood and MacConkey agar. The plates were incubated 18-24h and read according to standard quantitation protocols. Significant cultures were considered as those that contained urinary tract pathogens at amounts considered to reflect probable urinary infection. Urine was also sampled multiple times from the Uriswab to investigate any quantitative difference in sampling urine from the sponge over time.

RESULTS: 843 of the cultures (90\%) yielded equivalent results for quantitation $\left( \pm\right.$ one $\left.\log _{10}\right)$ and micro-organism isolated. There were $92(9.8 \%)$ discrepant results between the two systems where the quantitative difference was greater than one $\log _{10} ; 29(3.1 \%)$ had potential pathogens isolated. Of these, 10 were the same organism with the culture paddle (4) or Uriswab (6) having the higher count; 13 did not grow from the sponge and the remainder showed various discrepancies. 7 of 17 cultures grew at significant quantities from the dipslide only while the remaining 10 grew significant pathogens from the Uriswab only. The remaining 63 discrepant results $(6.7 \%)$ were not clinically significant: 34 of the 63 were lactobacilli or diphtheroids that did not grow from the preservative sponge. Multiple sampling over time did not alter the quantitation from the sponge cultures CONCLUSIONS: The two systems agreed in $90 \%$ of cases. Quantitative variation observed in a few cultures may be due to sampling error. The preservative sponge appeared to reduce the growth of common contaminants such as lactobacilli and diphtheroids. The clinical utility of this observation remains to be determined. The preservative sponge offers a simple, accurate and stable alternative for transport of urine for microbial culture.

\section{P29}

EVALUATION OF 3 CHROMOGENIC AGARS FOR THE RAPID IDENTIFICATION OF CANDIDA SPECIES H Adam ${ }^{2 *}$, S Boroumandi ${ }^{2}$, M Roscoe ${ }^{1}$, M Gris ${ }^{1}$, S Richardson ${ }^{1,2}$, Y Yau ${ }^{1,2}$

${ }^{1}$ Hospital for Sick Children, Toronto, ON; ${ }^{2}$ University of Toronto, Toronto, ON

OBJECTIVES: Conventional yeast identification requires evaluation of microscopic morphology and biochemical studies, which may take several days. Chromogenic media have recently been developed to facilitate simple and rapid yeast identification. Candida species cleave chromogenic substrates present in the agar producing colonies with unique colours. The purpose of this study was to evaluate 3 yeast chromogenic media: BBL
CHROMagar Candida (BBL), Bio-Rad CandiSelect 4 (Bio-Rad), and Oxoid OCCA (Oxoid) for the identification of common Candida species. METHODS: 39 Candida strains selected from a library of clinical isolates were included in the analysis. Study strains included C. albicans, C. glabrata, C. tropicalis, and C. krusei. A standard inoculum of each isolate was inoculated onto the 3 chromogenic media and incubated in accordance with the manufacturer's recommendations. Plates were examined at 24,48 , and 72 hours. Parameters evaluated in the study included accurate species identification, incubation time required for speciation, and ease of use.

RESULTS: At 24/48 hours, BBL, Bio-Rad, and Oxoid provided correct identification of $27 / 83,49 / 85$, and $0 / 67 \%$ of the strains, respectively. By 48 hours, BBL and Bio-Rad had correctly identified all of the C. albicans and C. krusei whereas Oxoid had only identified $92 \%$ of the C. albicans and $56 \%$ of the C. krusei strains. Bio-Rad is the only media approved for the identification of C. glabrata. It correctly identified $75 \%$ of C. glabrata strains by 48 hours.

CONCLUSIONS: BBL and Bio-Rad were found to be comparable in their identification of Candida species and were superior to Oxoid. Bio-Rad was advantageous as it provided greater identifications at 24 hours and is approved for C. glabrata; however, users preferred the BBL agar as the identification of Candida species was easier due a more distinct colour production profile.

\section{P30}

EVALUATION OF A NEW PROCESS FOR THE DETECTION AND REPORTING OF GROUP B STREPTOCOCCUS FROM VAGINORECTAL SPECIMENS

E Thomas, C Barth, K Hegglin*, D Brundage

Regina Qu'Appelle Health Region, Regina, SK

OBJECTIVE: Group B Streptococcus (GBS) is an important pathogen in neonates. The Centre for Disease Control (CDC) revised guidelines in 2002 recommend screening women for GBS carriage at 35 to 37 weeks gestation by culture of a vaginorectal swab using a selective broth with subculture onto a plate. The objective of our study was to 1) Evaluate the sensitivity of the recently implemented method for GBS detection. 2) Compile susceptibility results of GBS isolates. 3) Determine the impact on patient management of results delivery directly to the ward.

METHOD: Vaginorectal swabs are inoculated into StrepB Carrot Broth (Hardy Diagnostics) and incubated for 24 hours at $35^{\circ} \mathrm{C}$. Latex agglutination using Prolex Streptococcal latex agglutination (Prolab) is performed on broths with no color change. Broths positive for Group B antigen and orange colored broths are sub-cultured to Granada plates (Hardy Diagnostics) which are examined after 24 and 48 hours incubation at $35^{\circ} \mathrm{C}$ anaerobically. Orange colored colonies on the Granada plates are subcultured to Tryptic Soy Agar plates (TSA) with 5\% sheep blood (PML) and tested to confirm the presence of GBS. Susceptibility testing and the Dtest are performed by Kirby Bauer method.

RESULTS: From April 07-Oct. 071733 swabs were screened for the presence of GBS, with 361 cultures determined to be positive. Of the positive cultures, 249 broths turned orange following incubation, while 112 broths that showed no color change tested positive for Group B antigen. These 361 broths when cultured to Granada plates showed orange colonies in 360 and white colonies in 1 . All isolates were determined to be GBS; 1 non-hemolytic GBS was isolated. D-zone was present in 32 isolates. A copy of the GBS results was printed directly to the Labour and Birth Unit and sent to the physician's office.

CONCLUSION: The sensitivity of the carrot broth alone was $69 \%$, but when combined with a Granada plate the sensitivity increased to $99.7 \%$. This indicates the need for the dual testing method. Of the 361 GBS isolates, $9 \%$ showed inducible clindamycin resistance. Physicians and nurses indicate that reports sent directly to the ward provides information for appropriate antimicrobial use that is easily accessible and timely. 
P31

\section{PATTERN OF MRSA COLONIZATION IN FAMILIES OF CHILDREN WITH MRSA INFECTION}

S Wootton ${ }^{1 *}$, S Dobson ${ }^{1}$, E Thomas ${ }^{1}$, L Janz ${ }^{2}$, A Paccagnella ${ }^{2}$, S Goh ${ }^{2,1}$, L Hoang ${ }^{2}$

${ }^{1}$ University of British Columbia, Vancouver, BC; ${ }^{2} \mathrm{BC}$ Centre for

Disease Control, Vancouver, BC

OBJECTIVE: Staphylococcus aureus is a common cause of skin infections particularly among people who are colonized. The prevalence of $S$. aureus colonization depends on multiple factors including age, sex and ethnicity; children age 6-11 yrs are commonly colonized (prevalence $\sim 45 \%$ ). Information about patterns of MRSA colonization in children is limited. The goal of this study was to determine MRSA colonization patterns and strains in families of children with MRSA infection.

METHODS: Families of children age $0-16$ yrs with their first MRSA infection and who presented to BC Children's Hospital (Vancouver, BC) for evaluation (inpatient or outpatient) were eligible for enrolment during January-July 2007. MRSA isolates causing the child's initial MRSA infection were obtained. Families submitted monthly nasal cultures and questionnaires for 3 months. MRSA identification was performed by BC Children's Hospital, Microbiology Department and then isolates forwarded to BC Center for Disease Control, Laboratory Services for PFGE analysis.

RESULTS: 4 (28.6\%) out of 14 eligible families enrolled. MRSA was identified in 1 family (Family A) during the 3 month study period. Of the 3 members in Family A (patients A1, A2, A3), 2 were colonized with MRSA; A1 at 52, 105 and 143 days and A2 only at 143 days from enrolment (time of child's initial MRSA infection). Susceptibility patterns of the MRSA nasal isolates from Family A $(n=4)$ matched (erythromycin and clindamycin resistant). At the time of enrolment, MRSA was isolated from multiple sites in A1 (nose, neck, umbilicus, lip, right finger) and A2 (breast, vaginal swab). MRSA isolates from A1 and A2 obtained at enrolment had matching fingerprint patterns (CMRSA7). Only Family A experienced recurrent MRSA infection during the study period.

CONCLUSIONS: Tracking MRSA colonization in multiple family members can be time-consuming and inconvenient. By using a mail-in culture method, we were able to track colonization status in multiple families over time. Most families (75\%) were not colonized with MRSA during the study period. Matching MRSA nose isolates obtained from one family suggests possible transmission within families. CMRSA7 (USA400), isolated from Family A, is an unusual strain for western Canada.

\section{P32 \\ CHARACTERIZATION OF COMMUNITY-ASSOCIATED METHICILLIN-RESISTANT AND -SENSITIVE STAPHYLOCOCCUS AUREUS INFECTIONS IN THREE NORTHERN SASKATCHEWAN COMMUNITIES}

GR Golding ${ }^{1 *}$, MR Mulvey ${ }^{1}$, PN Levett ${ }^{3}$, RR Mcdonald ${ }^{2}$, J Irvine $^{3}$

${ }^{1}$ National Microbiology Laboratory, Winnipeg, MB; ${ }^{2}$ Saskatchewan Disease Control Laboratory, Regina, SK; ${ }^{3}$ Population Health Unit, LaRonge, SK and The Northern Antibiotic Resistance Partnership OBJECTIVE: The objective of this study was to establish a surveillance program for the characterization of methicillin-resistant Staphylococcus aureus (MRSA) and methicillin-sensitive Staphylococcus aureus (MSSA) infections acquired in remote communities.

METHODS: From July through December 2006, S. aureus isolates identified from skin and soft tissue, upper and lower respiratory, and urinary tract infections from three remote northern Saskatchewan communities were studied. Microbroth dilution and D-tests were used to determine susceptibilities to 14 antimicrobials according to CLSI guidelines. PFGE of SmaI digested genomic DNA was used for molecular characterization of the isolates. PCR was used to identify the presence of mecA, nuc, and PVL encoding genes.

RESULTS: 229 MRSA and 165 MSSA isolates were collected. 227 (99.1\%) of the MRSA infections were identified as Canadian PFGE epidemic type CMRSA7 (also referred to as USA400). The remaining two MRSA isolates were identified as CMRSA10 (USA300). PFGE banding patterns identified 12 different clusters of MSSA strains (SMSSA1-12) circulating within the three communities. In comparison to MSSA isolates, MRSA were more likely resistant to high levels of mupirocin $(\geq 256 \mathrm{mg} / \mathrm{L}) \quad(\mathrm{P} \leq 0.001)$, contain PVL $(\mathrm{P} \leq 0.001)$, and were associated more frequently with buttock $(\mathrm{P}=0.032)$ and chest/abdomen $(\mathrm{P} \leq 0.001)$ infections. MSSA however, were more likely resistant to erythromycin $(\mathrm{P} \leq 0.001)$, fusidic acid $(\mathrm{P} \leq 0.001)$, gentamicin $(\mathrm{P} \leq 0.001)$, low levels of mupirocin $(8-64 \mathrm{mg} / \mathrm{L})(\mathrm{P} \leq 0.001)$, displayed inducible clindamycin resistance $(\mathrm{P} \leq 0.001)$, and were more frequently associated with foot $(\mathrm{P} \leq 0.001)$ and urinary tract $(\mathrm{P}=0.008)$ infections.

CONCLUSION: The comparison of MRSA and MSSA infections in this study revealed many interesting phenotypic and genotypic differences, which requires further study. PVL positive community-associated strains of CMRSA7 and CMRSA10 are rapidly disseminating in communities throughout Canada. Future studies into the success, transmission, and virulence of these community-associated S. aureus strains are required. Extension of this surveillance program to include additional northern communities across Canada is warranted.

\section{P33}

COMPARISON OF COMMUNITY-ASSOCIATED

METHICILLIN-RESISTANT STAPHYLOCOCCUS AUREUS (CAMRSA) AND HOSPITAL-ASSOCIATED MRSA (HA-MRSA) IN CANADA: RESULTS FROM CANWARD 2007

KA Nichol ${ }^{1 *}$, MR Decorby ${ }^{2}$, PRS Lagace-Wiens ${ }^{2}$, M McCracken $^{3}$, MR Mulvey ${ }^{3}$, DJ Hoban ${ }^{1,2}$, GG Zhanel ${ }^{2}$

${ }^{1}$ Health Sciences Centre/Diagnostic Services of Manitoba, Winnipeg, MB; ${ }^{2}$ University of Manitoba, Winnipeg, MB; ${ }^{3}$ National Microbiology Laboratory, Winnipeg, MB

OBJECTIVES: Community-associated MRSA is an emerging pathogen. CA-MRSA strains differ from HA-MRSA in their genotypic and phenotypic characteristics. The purpose of this study was to compare the demographics, antimicrobial susceptibilities and molecular epidemiology of CAand HA-MRSA in Canada.

METHODS: Between January 2007 and present, 217 MRSA isolates were collected from Canadian patients affiliated with hospital outpatient clinics (OCs), emergency rooms (ERs), medical/surgical wards (MSWs) and intensive care units (ICUs). MRSA status was confirmed by multiplex PCR of the mecA and nuc genes. Susceptibilities to $\beta$-lactams, ciprofloxacin (CIP), clarithromycin (CLR), clindamycin (CLD), trimethoprim/sulfamethoxazole (SXT) and comparators were determined by broth microdilution according to CLSI guidelines. Strain typing was performed by PFGE and the presence of the Panton-Valentine leukocidin (PVL) gene was detected by PCR.

RESULTS: Of the 217 MRSA, 42 (19.4\%) were CA-MRSA and 175 $(80.6 \%)$ were HA-MRSA. Isolates (CA vs. HA) were obtained from patients in ERs (42.9 vs. $14.3 \%$ ), OCs (28.6 vs. $20.6 \%$ ), ICUs (14.3 vs. $18.3 \%$ ) and MSWs (14.3 vs. $46.9 \%$ ). MRSA (CA vs. HA) were isolated from wound/IV sites ( 54.8 vs. $17.7 \%$ ), blood (23.8 vs. $25.1 \%$ ), respiratory specimens ( 21.4 vs. $50.9 \%$ ) and urine (0.0 vs. $6.3 \%$ ). CA-MRSA belonged to PFGE types CMRSA10/USA300 $(n=27, \quad 64.3 \%)$ and CMRSA7/USA400 ( $n=15,35.7 \%)$; PFGE types identified among HAMRSA included CMRSA2/USA100/800 $(n=125,71.4 \%)$, CMRSA6 $(n=30,17.1 \%)$, CMRSA1/USA600 $(n=6,3.4 \%)$ and CMRSA5/USA500 $(n=3,1.7 \%)$. PVL was detected in 40/42 (95.2\%) CA-MRSA and 4/175 (2.3\%) HA-MRSA. Resistance rates (CA vs. HA) were 57.1 vs. $96.6 \%$ to CIP, 66.7 vs. $95.4 \%$ to CLR, 7.1 vs. $75.4 \%$ to CLD and 0.0 vs. $20.0 \%$ to SXT.

CONCLUSIONS: CA-MRSA represented 19.4\% of all MRSA isolates evaluated in this study. CA-MRSA were significantly more susceptible to CIP, CLR, CLD and SXT than HA-MRSA. No MRSA were resistant to vancomycin, linezolid, tigecycline or daptomycin. $95.2 \%$ of CA-MRSA were PVL(+) while most HA-MRSA were PVL(-) $(97.7 \%)$. The isolation of PVL(-)CA-MRSA and PVL(+)HA-MRSA, although rare, suggests that the epidemiology of MRSA in Canadian hospitals continues to change. 


\section{P34}

A REAL-TIME PCR ASSAY TO IDENTIFY MRSA AND CMRSA10 FROM SURVEILLANCE SAMPLES

G Ritchie*, A Wong, M Romney, S Champagne

Division of Medical Microbiology, St. Paul's Hospital, Providence

Health Care, Vancouver, BC

OBJECTIVE: Methicillin-resistant Staphylococcus aureus (MRSA) is a major nosocomial pathogen and an emerging community pathogen. At our hospital, the turnaround times (TAT) for culture are suboptimal (up to $72 \mathrm{~h}$ ). Rapid detection of MRSA colonization/ infection from surveillance samples is necessary for prompt implementation of infection control measures. A real-time PCR assay performed on culture isolates was designed to decrease TAT for reporting positive MRSA status, and to monitor the emergence of CMRSA10 in hospitalized patients using PVL as marker. METHODS: Two triplex PCR assays were developed on the Lightcycler. Both assays detected the mecA gene and the $l u k S$ gene responsible for PVL. To identify S. aureus the SA442 sequence was used in one assay and the nuc gene in the other assay. To assess the PCR assays, 183 well characterized clinical isolates were tested, including MRSA, (PVL positive and negative), methicillin susceptible $S$. aureus (MSSA), Coagulase Negative Staphylococci (CoNS), nonstaphylococcal bacterial isolates, and ATTC strains. Surveillance samples $(n=63)$ selected for MRSA screening were processed in parallel by culture and by PCR. Nasal, perineum, and open wound swabs were plated to MRSA-Select (Biorad) plates and incubated overnight. Suspicious colonies were sub-cultured on blood agar plates for identification according to conventional methods; concurrently, the same isolate was processed by PCR.

RESULTS: The SA442 PCR incorrectly identifying three isolates as CoNS. To avoid misidentification of these strains the SA442 was replaced by the nuc target to identify $S$. aureus. This second PCR yielded the expected results for all 183 clinical isolates. For the surveillance samples, the triplex PCR assay achieved $100 \%$ sensitivity and specificity for identifying MRSA. TAT for reporting swab samples could be reduced to $24 \mathrm{~h}$ by performing PCR on isolates recovered from MRSA chromogenic media. CONCLUSIONS: Triplex PCR performed on colonies growing from MRSA-Select after 18-24 h incubation is a rapid alternative to subculturing and culture characterization. TAT is reduced, which should allow for more rapid isolation of MRSA colonized/infected patients. The identification of PVL gene would permit surveillance for emerging CMRSA10.

\section{P35}

SURVEILLANCE FOR METHICILLIN-RESISTANT STAPHYLOCOCCUS AUREUS (MRSA) AT A TORONTO COMMUNITY SHELTER: MOLECULAR AND ANTIMICROBIAL RESISTANCE CHARACTERISTICS BM Willey ${ }^{1,2 *}$, B Borgundvaag ${ }^{1,3}$, T Svoboda $^{4}$, A Rostas ${ }^{1}$, P Gnanasuntharam ${ }^{1,3}$, M Loftus ${ }^{1}$, A Tyler ${ }^{1,3}$, V Porter $^{1}$, N Kreiswirth $^{1}$, L Louie ${ }^{5}$, A McGeer ${ }^{1,2,3}$

${ }^{1}$ Mount Sinai Hospital; ${ }^{2}$ University Health Network; ${ }^{3}$ University of Toronto; ${ }^{4}$ Centre for Research on Inner City Health, St Michael's Hospital; ${ }^{5}$ Sunnybrook and Womens College Health Science Centre, Toronto, ON

OBJECTIVES: Community-acquired (CA) MRSA has emerged rapidly in North America. In some regions, it has displaced methicillin susceptible S. aureus (MSSA) as the primary agent of skin and soft tissue infection (SSTI). While risk-factors for CA-MRSA infection have been well documented, little is known about colonization rates in high-risk individuals. METHODS: Surveillance was conducted between July 10 and August 18, 2007. During this time, 295 males frequenting a Toronto community shelter consented to provide swabs from their anterior nares and axilla, as well as from any visibly open wounds. These swabs were enriched in BHI broth, after which each was selectively cultured for MRSA using Denim Blue agar (Oxoid) and for MSSA using Mannitol Salt agar (Difco). Isolates were identified as MSSA or MRSA using standard methods. For all MRSA, antimicrobial susceptibility testing was performed using CLSI broth microdilution, typing was by standard Smal PFGE, while the SCCmec type and the presence of PVL were determined by PCR.
RESULTS: Overall, 122 (41\%) of residents carried S. aureus. Of these, 110 (37\%) carried MSSA and 12 (4.1\%) MRSA. When PFGE and susceptibility data were combined, it was clear the MRSA clustered into 5 groups, the largest comprising 7 residents positive from 11 sites that included a post-frostbite foot infection. This cluster, while unusually resistant $(\mathrm{R})$ to ciprofloxacin, clindamycin $(\mathrm{ermA} / \mathrm{C})$ and fusidic acid (MIC $>8 \mathrm{mg} / \mathrm{L}$, fusA), was otherwise indistinguishable from the epidemic SCCmec-IVa/PVL+/CMRSA-10/USA-300 community strain. Two further residents carried ciprofloxacin-R CMRSA-10/USA-300 typically R to erythromycin via the $m s r A$ gene. The 4 remaining MRSA were PVL negative and included 2 SCCmec-IVa CMRSA-2/USA-800 with R only to beta-lactams, 1 ciprofloxacin-R SCCmec-IVa CMRSA-1/USA-600 with msrA-mediated erythromycin-R, and 1 SCCmec-II CMRSA-2/USA-100 with a susceptibility profile typical of nosocomial acquisition.

CONCLUSION: Interestingly, four separate CA-MRSA clusters derived from three distinct genetic lineages, all harbouring the community-associated SCCMEC-IVa cassette, were identified in this one Toronto shelter. Of concern is that the predominant strain was an unusually drug resistant variant of the epidemic CMRSA-10/USA-300. The implications of colonization with this strain are yet to be determined.

\section{P36}

\section{EPIDEMIOLOGY OF TINEA PEDIS (TP) AND TOENAIL ONYCHOMYCOSIS (TO) IN SASKATCHEWAN (SK), CANADA}

\section{H Congly*}

Saskatchewan Disease Control Laboratory, Saskatchewan Health,

Regina, SK

OBJECTIVE: TP and TO are fungal foot diseases of public health importance due to the risks of spread of infectious fungal elements to others. There is little epidemiological information on pedal dermatomycoses in SK. This survey aimed to characterize the epidemiology of TP and TO in SK for 2005 and 2006.

METHODS: The demographic, clinical and mycological data between 2005 and 2006 were retrospectively analyzed. Fungi were identified by conventional laboratory methods. TP was established by hyphae seen in $\mathrm{KOH}$ examination of skin and dermatophyte cultures. TO was confirmed by hyphae and/or yeast cells and pseudohyphae observed in $\mathrm{KOH}$ smears of nails as well as dermatophyte and/or yeast cultures.

RESULTS: Of the 178 pedal skin specimens, 39 (21.9\% incidence) were positive for dermatophytes. Trichophyton rubrum (TRR) (66.7\%), Trichophyton mentagrophytes (TRM) (28.2\%) and Epidermophyton floccosum $(5.1 \%)$ were identified. TP was more common in males than females (M:F=1.8:1) and in the 40-79 age group (69.2\%). TRM and TRR occurred in $82 \%$ and $50 \%$ of TP cases, respectively, in the summer. Of the 1300 toenail samples, 244 (18.8\% incidence) were positive for dermatophytes and Candida (C). TRR (70.9\%), TRM (28.2\%), Microsporum canis $(0.4 \%)$, Microsporum persicolor $(0.4 \%)$, C. parapsilosis $(66.7 \%)$ and C. albicans $(33.3 \%)$ were the species encountered. TO was more common in males $(62.3 \%)$ than females $(37.7 \%)$ and in the $40-79$ age group $(72.5 \%)$. TRM occurred in $64.7 \%$ of TO cases in the winter, whereas TRR in $54.8 \%$ of cases in the summer.

CONCLUSIONS: The findings in this survey are consistent with other studies in North America with TRR being the most common and TRM the second most common causes of TP and TO. The incidences of both TP and TO increase with age. TP caused by TRM is a summer disease. TO caused by TRM is twice as common in winter than summer. 
P37

ANOTHER LETTER IN THE VAN ALPHABET; MOLECULAR CHARACTERIZATION OF THE NOVEL D-ALANINE-DSERINE OPERON VANL FROM A VANCOMYCIN-RESISTANT ENTEROCOCCUS FAECALIS ISOLATED IN CANADA

D Boyd $^{1 *}$, B Willey ${ }^{2}$, D Fawcett ${ }^{3}$, N Gillani ${ }^{3}$, M Mulvey ${ }^{1}$

${ }^{1}$ Public Health Agency of Canada, Winnipeg, MB; ${ }^{2}$ Mt. Sinai Hospital and University Health Network, Toronto, ON; ${ }^{3}$ Rouge Valley Health Centre Centenary Site, Scarborough, ON

OBJECTIVES: To characterize the mechanism of vancomycin resistance in Enterococcus faecalis N06-0364 (vancomycin MIC of $8 \mu \mathrm{g} / \mathrm{mL}$ ).

METHODS: PCR was used to assay for $\operatorname{van} A, B, C, D, E$, and $G$. Antimicrobial susceptibilities were by Vitek II AST-GP63 card, CLSI broth microdilution, and Etest. Standard and TAIL PCR with sequencespecific and degenerate primers was used to isolate the VanL operon and the products characterized by dideoxy sequencing. Homology searches were with the BLAST suite at NCBI website (www.ncbi.nlm.nih.gov/BLAST/)

RESULTS: E. faecalis N06-0364 was isolated from a single patient on VRE screening 2 days after admission for surgery. This VRE strain was not isolated from any other patient in the community hospital or again from this patient on re-screening 7 days later. By PCR, it was negative for vanA, $B, C, D, E$, and $G$, yet it had a vancomycin MIC of $8 \mu \mathrm{g} / \mathrm{mL}$ by broth microdilution and Etest. The sequence of a product obtained with degenerate primers for D-Ala-D-Xxx ligases showed $57 \%$ identity to the VanC ligase and this was designated VanL. Using PCR and sequencing, an 8,295 bp region of the N06-0364 genome harbouring the VanL operon was characterized. The operon was similar to the VanC operon in organization but the VanT serine racemase was encoded by 2 genes, vanTmL encoding a membrane-binding region, and vanTrL encoding the racemase region. Overall the VanL protein exhibited $51 \%, 49 \%$, and $42 \%$ identity to VanE, VanC, and VanG D-Ala-D-Ser ligases, respectively, and 35\%, 37\%, and $40 \%$ identity to VanA, VanB, and VanD2 D-Ala-D-Lac ligases, respectively. An insertion sequence, ISEnfa364, was found downstream of the VanL operon. Transfer of VanL to a susceptible E. faecalis strain could not be demonstrated.

CONCLUSIONS: This study characterized a novel vancomycin resistance operon, VanL, isolated from a clinical E. faecalis strain. This extends the van alphabet of resistance genes in enterococci which includes the $\operatorname{van} A, B$, and D D-Ala-D-Lac ligases which confer moderate to high-level vancomycin resistance, and the $\operatorname{vanC}, E, G$, and $L$ D-Ala-D-Ser ligases which confer low-level vancomycin resistance.

\section{P38}

WORMS AND WAR: A CASE REPORT OF TRAUMA AND TROPICAL DISEASE INTERSECTING

M Hawkes $^{1 *}$, C Masumbuko $^{2}$

${ }^{1}$ University of Toronto, Toronto, ON; ${ }^{2}$ HEAL Africa, Goma, North

Kivu, the Democratic Republic of the Congo

OBJECTIVE: Ascaris lumbricoides is the most common human intestinal nematode, infecting an estimated 1 billion people worldwide. Many areas of the world affected by violent conflict, such as the Democratic Republic of the Congo, also carry a high burden of tropical diseases, including ascariasis. We report a case and present images of heavy intestinal infestation with Ascaris lumbricoides complicating the surgical management of a gunshot injury to the abdomen and draw attention to the complex burden of illness among children living in areas of violent conflict.

METHODS: Case report and review of the literature.

RESULTS: A 12 year old girl presented with gunshot wounds to an emergency unit in Goma, Democratic Republic of Congo. After resuscitation, the patient was urgently brought to the operating suite for exploratory laparotomy for management of penetrating abdominal trauma, at which point the operating team was startled to discover numerous live adult worms in the abdomen. A total of 22 ascarids were extracted one by one through a traumatic enterorotomy wound and from the peritoneal cavity. Surgical repair included approximation and closure of damaged gastric wall, resection of injured jejunum $(50 \mathrm{~cm})$ and ileum $(70 \mathrm{~cm})$ with end-to-end re-anastomosis of the small bowel. The patient recovered from the abdominal surgery without complication, having received broad spectrum antibiotics for intraabdominal infection prophylaxis, as well as mebendazole post-operatively to eradicate any remaining ascarids.

CONCLUSION: This is the first report, to our knowledge, of helminthic infestation complicating abdominal trauma surgery. Co-existing pathologies in this interesting case highlight in the dual burden of violence and infectious diseases experienced by children living in areas of violent conflict.

\section{P39}

VALIDATION OF RT-PCR METHODS FOR MOLECULAR DIAGNOSTICS AND EPIDEMIOLOGY OF MUMPS VIRUS

J Hiebert $^{1 *}$, C Fung ${ }^{3}$, C Sevenhuysen ${ }^{1}$, J Beirnes ${ }^{1}$, T Hatchette $^{2}$, G Tipples ${ }^{1}$

${ }^{1}$ National Microbiology Laboratory, Public Health Agency of

Canada, Winnipeg, MB; ${ }^{2}$ Department of Pathology and Laboratory

Medicine, QE II Health Science Center, Halifax, NS; ${ }^{3}$ Biological

Sciences, University of Alberta, Edmonton, AB

OBJECTIVE: A mumps vaccine has been licensed for use in Canada since 1969 resulting in a dramatic decrease in the number of cases per year. None the less, outbreaks of mumps have sporadically occurred, the most recent in Nova Scotia and other provinces in the spring of 2007. RT-PCR for mumps is useful for both diagnostic and molecular epidemiologic purposes. Our objective was to validate a sensitive and rapid RT-PCR method for use as a mumps molecular diagnostic method.

METHODS: Two real-time TaqMan probe based RT-PCR assays for the detection of mumps virus RNA in clinical specimens were selected from the literature for evaluation in addition to our genotyping RT-PCR method. Dilution series of synthetic F and SH gene RNA were tested a minimum of 21 times with all three RT-PCR methods to determine their detection limits, reproducibility and repeatability. In addition, a blinded panel consisting of 43 clinical specimens that had been previously tested by another centre were tested.

RESULTS: The detection limit, as defined as the minimum number of target copies that produce a positive result in $95 \%$ of test runs, was found to be 10 and 10000 copies of RNA per reaction for the F and SH real-time RT-PCRs respectively. The detection limit for the genotyping RT-PCR was found to be 100 copies. The detection limit concentration was positive every time that it was tested (minimum of 7 separate runs of triplicates) resulting in reproducibility and repeatability estimates of $>95 \%$. Finally, the panel of 43 samples tested yielded the expected results for all samples ( 23 negative and 20 positive). The sensitivity and specificity of the assay are therefore estimated to be $>95 \%$.

CONCLUSION: The SH real-time RT-PCR did not perform in our hands as published in that it was not analytically sensitive. Therefore it was determined not to use it as part of our diagnostic mumps RT-PCR assay. On the other hand, the published F real-time RT-PCR proved to be fast, analytically sensitive, reproducible and have high specificity and sensitivity.

\section{P40}

COMPARISON OF VITEK2 AST-YSO1 CARD, SENSITITER YEASTONE BROTH MICRODILUTION, AMPHOTERICIN ETEST, FLUCONAZOLE DISK DIFFUSION (DD) WITH THE CLSI REFERENCE METHOD BROTH MACRODILUTION (BMD) FOR ANTIFUNGAL SUSCEPTIBILITY TESTING OF CANDIDA SPP

S Champagne*, S Pengilly, M Dumouchelle, M Romney

Providence Health Care, Vancouver, BC

OBJECTIVES: Susceptibility testing of Candida spp. using reference method BMD is labour intensive and requires technical expertise. Results are sometimes subject to interpretation. We compared four alternative methods of antifungal susceptibility testing, including the new Vitek2 antifungal yeast card, the Sensititer YeastONE system, amphotericin B Etest, and fluconazole DD. The objective was to find a simpler, more rapid and accurate alternative method to BMD. 
METHODS: We performed antifungal susceptibility testing on 40 clinical isolates of Candida spp. The Vitek card (AST-YS01) for amphotericin B, flucytosine, fluconazole and voriconazole antifungal testing was evaluated. The colorimetric YeastONE system included the same antifungals and was read by experienced technologists at $24 \mathrm{~h}$. Amphotericin B Etest was applied to agar plates with RPMI medium and read at $24 \mathrm{~h}$. Fluconazole DD and BMD were performed according to CLSI document M27-A guidelines.

RESULTS: Category agreement (CA) was analysed for fluconazole, flucytosine and voriconazole compared to BMD using CLSI breakpoints at 24 and $48 \mathrm{~h}$. At $24 \mathrm{~h}$, fluconazole CA was $79.0 \%$ for Vitek2 and $74.4 \%$ for both YeastONE and DD; at 48 h, CA was $77.5 \%$ for Vitek2, YeastONE and DD. Flucytosine CA for Vitek 2 was $100 \%$ and $97.5 \%$ at 24 and 48 h, respectively, while CA for YeastONE was $95 \%$ and $90 \%$. CA for voriconazole performed better at $24 \mathrm{~h}$ with $89.7 \%$ for Vitek2 and $89.7 \%$ for YeastONE; $48 \mathrm{~h}$ reading showed decreased CA with $79.5 \%$ for both Vitek2 and YeastONE. The average time to reporting for Vitek2 AST-YS01 was $16.2 \mathrm{~h}$. Although there is no defined breakpoint for amphotericin B susceptibility testing, the MICs for all isolates were $\leq 1 \mathrm{mg} / \mathrm{L}$. Amphotericin $\mathrm{B} \mathrm{MIC}_{50}$ values for Vitek2, YeastONE, Etest and BMD were 0.5, 0.5, 0.094 and $0.03 \mathrm{mg} / \mathrm{L}$, respectively, while $\mathrm{MIC}_{90}$ values were $1.0,0.95,0.5$, and $0.12 \mathrm{mg} / \mathrm{L}$.

CONCLUSIONS: Vitek2 AST-YSO1 and Sensititer YeastONE appear to be good alternatives to BMD for antifungal testing. Both methods provide higher MICs to amphotericin B compared to BMD; amphotericin B testing by Etest may be a more accurate method. Vitek2 AST-YS01 provides an objective reading and more rapid results than Sensititer YeastONE. Further studies are needed, especially for non-albicans Candida spp.

\section{P41}

\section{ASSESSMENT OF QUIKICULT (IME INC) (QC), A RAPID CULTURE-BASED SCREENING METHOD FOR URINE SPECIMENS (UR)}

K Tan ${ }^{1,2}$, S Harrison 1, J Reid ${ }^{1}$, D Roscoe ${ }^{1,2}$

${ }^{1}$ Vancouver General Hospital, Vancouver, BC; ${ }^{2}$ Canada, University of British Columbia, Vancouver, BC

OBJECTIVE: UR are commonly received for culture in microbiology. Culture requires overnight incubation to assessment of positivity, yet many are culture negative. A screening method to detect positive samples that require further investigation would eliminate further processing of negative UR. The QC is a culture-based rapid screen method based on turbidity of the specimen prior to and after a 3 hour incubation in nutrient broth. This study evaluated QC compared to routine culture methods.

METHODS: Culture was done using a $0.001 \mathrm{~mL}$ loop to inoculate a sheep blood and MacConkey agar bi-plate. Positive cultures were defined as growth $>100$ million CFU/L after overnight incubation. QC was performed according to manufacturer's instructions within 2 hours of culture. The QC ampoule contains freeze-dried media and draws $7 \mathrm{~mL}$ UR when vacuum seal is broken. UR with less than $7 \mathrm{~mL}$ required dilution of $2.5 \mathrm{~mL}$ UR into $5 \mathrm{~mL}$ solution. Ampoules were incubated at $35^{\circ} \mathrm{C}$ for $30 \mathrm{~min}$ and baseline turbidity read in the provided reader. Specimens were re-incubated for $3 \mathrm{hrs}$ and re-read. A high turbidity at baseline or a significant increase of turbidity after incubation is a positive QC result. Data were automatically transferred to the QC software and compared to culture results.

RESULTS: 523 UR were assessed, of which 123 (24\%) were positive by culture. Overall QC performance compared to culture was: $85 \%$ sensitivity (SE) and $83 \%$ specificity (SP). 147 specimens required dilution; SE of these UR was $81 \%$ compared to the SE of undiluted UR of $85 \%$. For 75 UR with culture growth $<100$ million CFU/L, QC SE was $24 \%$. UR positive by QC but with insignificant or no growth on culture were noted to be turbid or contained blood on visual inspection of the UR. Six false negative UR were culture positive for Pseudomonas sp or yeast, which disproportionately represents these organisms when compared with true positive UR.

CONCLUSIONS: QC performance is dependent on the interpretation of a significant urine culture. In our evaluation there was a marked decrease in SE of QC to detect UR with growth of $<100$ million CFU/L. Low volume UR require additional handling but this could be decreased by ward education. QC is easy to perform and can be accommodated into the laboratory routine; procedural automation would potentially enhance its value.

\section{P42}

MOLECULAR EPIDEMIOLOGY OF A MULTICLONAL

OUTBREAK OF K. PNEUMONIAE AND E. COLI HARBORING PLASMID-MEDIATED DHA-1 AND QNRB4 FROM A TORONTO COMMUNITY HOSPITAL

D Boyd ${ }^{1}$, B Willey ${ }^{2 *}$, D Fawcett ${ }^{3}$, N Gillani ${ }^{3}$, M Mulvey ${ }^{1}$

${ }^{1}$ Public Health Agency of Canada, Winnipeg, MB; ${ }^{2}$ Mt. Sinai Hospital and University Health Network, Toronto, ON; ${ }^{3}$ Rouge Valley Health Centre Centenary Site, Scarborough, ON

OBJECTIVES: In 2006 a cluster of patients was identified that carried $K$. pneumoniae and/or E. coli harbouring bla $a_{\mathrm{DHA}-1}$. Retrospective analysis identified two patients from 2001/2002 that carried K. pneumoniae(DHA) and E. coli (DHA) one of whom was still in the hospital. Genomic fingerprints of the strains were diverse. Further genome fingerprinting and analysis of the $b l a_{\text {DHA-1 }}$ plasmids was carried out.

METHODS: Genomic fingerprinting was done using pulsed-field gel electrophoresis. PCR with specific primers was used for identification of $b l a_{\mathrm{DHA}}$ and $q n \mathrm{rB}$. PCR mapping was done based on previously published plasmidic-bla $a_{\mathrm{DHA}}$ regions. RFLP was done with EcoRI.

RESULTS: $31 \mathrm{~K}$. pneumoniae with 11 unique fingerprints and $20 \mathrm{E}$. coli with 10 unique fingerprints were characterized from 29 patients. 50/51 strains were positive for bla $a_{\mathrm{DHA}}$ and qnrB4. Plasmid RFLP from different fingerprint types indicated that $10 / 11 \mathrm{~K}$. pneumoniae harboured plasmid type D1 or a variant and 8/9 E. coli harboured plasmid type D1 or a variant. One patient carried a K. pneumoniae and an E. coli both harbouring plasmid type D3. The index patients from 2000/2001 were found to still be carrying K. pneumoniae and E. coli strains harboring plasmid type D1 in 2005. Plasmid RFLP and PCR mapping indicated that plasmid type D1 exhibited extensive similarity to a plasmid found in K. pneumoniae strains isolated in Paris, France between 1999 and 2003.

CONCLUSIONS: Strains from a multiclonal outbreak of K. pneumoniae and E. coli from a chronic care ward were found to carry a related plasmid harboring $b l a_{\mathrm{DHA}-1}$ and $q n r B$. The index strains have been in the institution since at least 2000. The plasmid is highly similar to one found in Paris, France. This is the first outbreak involving bla $a_{\text {DHA-1 }}$ in North America.

\section{P43}

FATAL CASES OF STAPHYLOCOCCUS AUREUS PLEURAL EMPYEMA IN INFANTS

A Rougemont, C Buteau, P Ovetchkine, JC Fournet, C Bergeron, D Bouron-Dal Soglio

CHU Sainte Justine, Montreal, QC

OBJECTIVE: Community-acquired lower respiratory tract infections due to Staphylococcus aureus (S. aureus) are on the rise especially since the introduction of the pneumococcal vaccine. The virulence of staphylococcal strains is notably determined by different genes, such as the PantonValentine leukocidin (PVL) gene, expressed in S. aureus isolates obtained from pediatric necrotizing pneumonia samples. We describe two similar cases of infants less than one year old with an upper respiratory tract infection quickly followed by severe respiratory distress and death, having occurred in the same city and during the same winter.

METHODS: Necropsies performed between November 2006 and March 2007 revealed bronchopneumonia and an important pleural empyema, justifying the review of the clinical chart and laboratories exams.

RESULTS: An oxacillin-sensitive S. aureus carrying the PVL gene was identified in both cases.

CONCLUSION: These observations including a third case with similar necropsies results stress the need for every physician to recognize these severe and increasingly frequent $S$. aureus infections in young children and to report such cases to local public health autorities. Until now, only Vancomycin-resistant Staphylococcal infection have to be report to the Provincial Public Health Laboratories. 


\section{P44}

IMPORTED CASES OF CHIKUNGUNYA VIRUS-ASSOCIATED DISEASE AMONG CANADIAN TRAVELLERS: 2005-2007

K Makowski*, M Andonova, H Artsob, M Drebot National Microbiology Laboratory, Winnipeg, MB

OBJECTIVES: Since 2005 an outbreak of Chikungunya virus (CHIKV) in India and the islands of the Indian Ocean has resulted in over a million human infections. The objective of this study was to identify CHIV associated illness among Canadian travellers returning from the Indian Ocean region by serological and PCR-based diagnostics.

METHODS: Suspect cases of CHIKV were identified based on clinical symptoms and travel history. Serum from patients was tested by hemagglutination inhibition (HI) serology and IFA using CHIKV antigen generated from mice and tissue culture. Conventional and real-time RT-PCR specific for the envelope gene was utilized to amplify and detect viral RNA.

RESULTS: Imported cases of CHIKV associated illness among Canadian travellers to this region were identified as early as August of 2005. As of November 1, 2007 a total of 33 individuals from seven different provinces (Nova Scotia-1, Quebec-8, Ontario-16, Manitoba-4, Saskatchewan-1, Alberta-2, and British Columbia-1) have been diagnosed with CHIKV associated disease. Patients exhibited typical symptoms and disease manifestations associated with CHIKV infections including febrile illness, polyarthritis, and rash. Travel history included visits to the island of Reunion, Mauritius, Seychelles Islands, Madagascar, and India. Cases were identified using HI and IFA serology and PCR-based diagnostics. HI titres ranged from 40 to 2560 and included a number of seroconversions. Phylogenetic analysis of amplified CHIKV RNA from acute sera will be presented.

CONCLUSIONS: The ongoing nature of the CHIKV outbreak in the Indian Ocean region and recently in Italy highlights the continued need for travel advisories informing the public of the risk of exposure to this agent and the timely testing of patients who have visited these geographical areas.

\section{P45}

ONGOING CONTROL OF HAEMOPHILUS INFLUENZAE B INFECTIONS IN CANADIAN CHILDREN: 2004-2007

D Scheifele $^{* 1,2}$, S Halperin ${ }^{1,2}$, B Law ${ }^{1,3}$, R Bortolussi ${ }^{1,2}$ ${ }^{1}$ Canadian Immunization Monitoring Program, Active (IMPACT), Ottawa, ON; ${ }^{2}$ Canadian Paediatric Society, Ottawa, ON; ${ }^{3}$ Public Health Agency of Canada, Ottawa, ON

OBJECTIVE: Since 1998 all Canadian provinces have supplied the same vaccine to prevent Haemophilus influenzae type b (Hib) infections, a PRPtetanus protein (PRP-T) conjugate given in combination with DTaP.IPV vaccines (Pentacel®, sanofi pasteur vaccines). The uniformity of Hib vaccination over time affords an opportunity to observe the ongoing effectiveness of the vaccine, including any effects of concurrent pneumococcal vaccinations (added in 2003-2005) or of increasing time since vaccination.

METHODS: Active surveillance for culture-confirmed invasive Hib infections was conducted at 12 pediatric centers across Canada. These centers extend from coast to coast and represent over $90 \%$ of the nation's tertiary care pediatric beds. A nurse monitor is employed at each center to actively seek and report target conditions. Case-finding included laboratory and health records reviews. Immunization history was obtained for each case. Surveillance procedures for Hib have been uniform since 1994. RESULTS: Cases totals were: $2004-9,2005-5,2006-9,2007-0$ (to November 15). 15/23 cases were incompletely immunized (too young, 11) or unimmunized by parent choice (4). Vaccine failures totalled $3(0-1 / \mathrm{yr})$ after 3 doses and $5(0-4 / y r)$ after 4 doses. Two of the latter cases were immunocompromised. All but one of the vaccine failures were $\leq 6$ years old, the older exception (age 9) being immunocompromised. Only 4 cases were preventable, indicating that the annual disease burden is nearing the irreducible minimum.

CONCLUSIONS: Recent case totals were unchanged from 1998-2003 when the same vaccine was used and annual case totals were 3-16 (average 8.8 ), with 1-3 vaccine failures. Concurrent pneumococcal vaccination of infants has had no apparent impact on short-term effectiveness of Hib vaccination. There is no indication of waning protection as the oldest PRP-T vaccinated children enter their mid-teen years. The current vaccine is highly effective and rarely associated with vaccine failures. The current disease burden is $<2 \%$ of the 485 admissions reported by IMPACT in 1985 prior to Hib vaccine introduction.

\section{P46}

\section{CRYPTOSPORIDIOSIS IN BEARS IN METRO VANCOUVER WATERSHEDS: WHAT ARE THE PUBLIC HEALTH IMPLICATIONS?}

S Shay ${ }^{1}$, R Grover ${ }^{2}$, A Li ${ }^{1}$, B Auk ${ }^{1 *}$, J Fung ${ }^{1}$, J Isaac-Renton ${ }^{1,2}$, C Ong ${ }^{1,2}$

1Environmental Microbiology, BC Centre for Disease Control, Vancouver, BC; ${ }^{2}$ Department of Pathology \& Laboratory Medicine, University of British Columbia, Vancouver, BC

OBJECTIVE: An increase in bear fecal specimens with Cryptosporidium spp. oocysts has been observed in longitudinal surveillance studies on wildlife in the watersheds of Metro Vancouver. Cryptosporidiosis is a zoonotic disease and the drinking water supply for Metro Vancouver is presently treated only by chlorination and/or ozonation. These methods of disinfection are inadequate for the inactivation of waterborne protozoan parasites. The aim of this study was to identify the species of Cryptosporidium that are prevalent in the bear population of these watersheds. Given the broad host range of certain Cryptosporidium species, these results would allow us to ascertain if the current epizootic of ursine cryptosporidiosis in the Metro Vancouver watersheds has any potential impact on public health in the Lower Mainland region of British Columbia.

METHODS: Cryptosporidium oocysts were isolated from fecal specimens that were identified morphologically as originating from bears in the Metro Vancouver watersheds. The 18S rRNA gene was amplified from genomic DNA using nested PCR and sequenced bi-directionally by cycle sequencing. The Cryptosporidium species was assigned by comparison of assembled sequences to GenBank reference sequences using the BLAST search tool.

RESULTS: The 18S rRNA gene was amplified and sequenced successfully from three ursine fecal specimens. All specimens had DNA sequences that were identical to a Cryptosporidium 18S rRNA gene sequence obtained from a black bear in Virginia. To date no human infections have been reported with this particular species of Cryptosporidium.

CONCLUSION: The identification of a species of Cryptosporidium that is non-pathogenic to humans in the fecal specimens of 3 infected bears in areas that supply drinking water to municipalities in Metro Vancouver indicates that the potential risk of zoonotic spread by waterborne transmission of cryptosporidiosis is likely to be low or negligible. In order to continue on with this favourable assessment, further surveillance and screening for cryptosporidiosis in bears as well as other watershed wildlife will be necessary until the filtration plants under construction are put into service.

\section{P47} CANAVANINE-GLYCINE-BROMOTHYMOL BLUE MEDIA
VERSUS RFLP FOR THE IDENTIFICATION OF C. NEOFORMANS VAR. GATTII, AN EMERGING PATHOGEN IN BRITISH COLUMBIA

S Mithani ${ }^{*}$, M Lee ${ }^{1}$, G Al-Rawahi ${ }^{2}$, M Morshed ${ }^{1,3}$, J Isaac-

Renton $^{1,3}$, L Hoang ${ }^{1,3}$

${ }^{1}$ Laboratory Services, BC Centre for Disease Control, PHSA, Vancouver, BC; ${ }^{2}$ Children's \& Women's Health Centre of British Columbia, Vancouver, BC; ${ }^{3}$ Department of Pathology and Laboratory Medicine, University of British Columbia, Vancouver, BC

OBJECTIVE: An outbreak of Cryptococcus neoformans var. gattii (C. gattii), first isolated on Vancouver Island in 1999, has spread to the mainland of BC. Cases have also emerged in Washington State and Oregon, USA. Recognition of C. gattii versus other varieties of C. neoformans is essential for epidemiological tracking and patient management. Currently, CGB (Canavanine-Glycine-Bromothymol Blue) media is routinely used as a 
first line screening method. All CGB positive isolates are subtyped using a $\mathrm{BC}$ developed RFLP method to provide gold-standard genotypic identification. The objective of this study is to determine the sensitivity and specificity of CGB agar for phenotypic identification versus RFLP for the identification of C. gattii and C. neoformans var grubii/neoformans.

METHODS: A retrospective study was conducted on 50 isolates of C. neoformans var grubii/neoformans and 86 isolates of C. gattii. The phenotypic results using the CGB agar and genotypic results using RFLP as the reference standard were compared for each isolate.

RESULTS: The sensitivity of the CGB agar was $97.6 \%$, that is, out of the 86 C. gattii isolates, 84 were CGB positive and subtyped as "VG" strains. Two isolates were CGB negative but subtyped as "VGIIa", one of the most common genotypes seen on Vancouver Island. All of the C. neoformans var grubii/neoformans isolates were CGB negative and molecularly subtyped as "VN" stains, providing a specificity for the CGB agar of $100 \%$. CONCLUSION: These results indicate that with the emergence of CGB negative strains of C. gattii, the continued use of the CGB plates alone may result in missing cases of C. gattii infection. At BCCDC Laboratory Services all submitted isolates of $\mathrm{C}$. neoformans receive CGB screening and RFLP as confirmatory genotyping test. Phenotypic screening methods such as CGB are valuable for the presumptive identification of C. gattii, however, in cases where there is a high suspicion of C. gattii infection epidemiologically and clinically, genotyping confirmation should be performed. Identification of all C. gattii cases is important for monitoring geographic spread of this emerging pathogen in North America.

\section{P48}

FIRST ISOLATION OF BORRELIA BURGDORFERI FROM IXODES COOKEI REMOVED FROM A DOG IN ALBERTA K Fernando $^{1 *}$, M-K Lee ${ }^{1}$, Q Wong ${ }^{1}$, K Burgess ${ }^{2}$, L Durden ${ }^{3}$, M Morshed ${ }^{1,4}$

${ }^{1}$ BC Centre for Disease Control, Vancouver, BC; ${ }^{2}$ St. Albert Animal Clinic, St. Albert, AB; ${ }^{3}$ Georgia Southern University, Statesboro, GA, United States; ${ }^{4}$ University of British Columbia, Vancouver, BC OBJECTIVE: The Borrelia spirochete infected tick found on a dog in the Edmonton area (St. Albert) raised the concerns of Lyme disease in Alberta in the spring of 2007. This study provides detailed information of the laboratory findings on this occurrence and recommendations for further research in this area.

METHODS: The tick was identified using a stereo microscope. BSK-H media was used to isolate and culture the Borrelia spirochete. To monitor growth in culture, microscopic examination was performed directly using dark field microscopy on a weekly basis for up to 4 weeks. Dog serum was tested using immunofluorescent assays (IFA) with Borrelia burgdorferi B31 (ATCC 35210) coated slides and confirmed with IgM and IgG Western Blots. PCR was performed by amplifying both the $r r f-r r l$ and ospA regions using the Stratagene Robocycer. The $r r f-r r l$ amplicon was sequenced with ABI 3130 and subsequent cluster analysis was performed with MegAlign (DNAStar) software.

RESULTS: Carrier: Dog serum was positive against anti B. burgdorferi antibodies by both IFA and Western Blot methods.

Vector: Identified as Ixodes cookei.

Pathogen: The Borrelia spirochete was isolated in culture using mid gut samples from the tick.

Molecular: PCR amplification of the $r f-r r l / o s p A$ regions of the isolate and cluster analysis suggested that this isolate belonged to Borrelia burgdorferi senso stricto.

CONCLUSION: This is the first report of Borrelia burgdorferi isolated from an Ixodes cookei tick found on an Albertan dog. The positive serology result from the host and the successful isolation of the Borrelia spirochete from the vector confirmed the presence of B. burgdorferi in the Edmonton area. PCR amplification on both the $r r f-r r /$ ospA regions and subsequent cluster analysis suggested the isolate belonged to the Borrelia burgdorferi senso stricto genogroup, which is responsible for Lyme disease in humans. Further surveillance in the Edmonton area is recommended.

\section{P49}

A RARE CRYPTOSPORIDIUM HOMINIS SUBTYPE LINKS CRYPTOSPORIDIOSIS CASES ASSOCIATED WITH THE USE OF A COMMUNITY AQUATIC CENTRE

C Plohman 1', S Chow ${ }^{1}$, R Gustafson 1,3, R Parker², C Ong ${ }^{1,3 *}$

${ }^{1}$ University of British Columbia, Vancouver, BC; ${ }^{2}$ Fraser Health

Authority, BC; ${ }^{3}$ BC Centre for Disease Control, Vancouver, BC

OBJECTIVE: An increased number of cryptosporidiosis occurred in December 2003 in the Tri-Cities area of the Lower Mainland region in BC. Although all cases were associated with the use of a community aquatic centre, their onset dates were spread over an extended period of 3 months. The aim of this study was to use molecular methods to type the cryptosporidiosis cases and confirm that the cases in this disease cluster were related.

METHODS: Fecal specimens were collected from laboratory-confirmed cryptosporidiosis cases with histories of exposure to the implicated aquatic centre. Genomic DNA was extracted from purified Cryptosporidium oocysts and the species and subtype determined by PCR amplification and sequencing of the $18 \mathrm{~S}$ rRNA and gp60 genes. The DNA sequences of amplicons were determined by cycle sequencing, assembled and the gp60 allele was obtained by multiple sequence alignment with Genbank reference sequences. The subtype was identified by visually quantifying the number of microsatellite repeats and carrying out a phylogenetic analysis using all available gp60 Id subtype sequences.

RESULTS: Nine fecal specimens were collected from laboratory confirmed cryptosporidiosis cases. The $18 \mathrm{~S}$ rRNA and gp60 genes were amplified successfully from 4 of 5 specimens selected for testing using the criterion that they were not from cases in the same house-hold. All cases were infected with Cryptosporidium hominis. The gp60 sequences from all 4 cases were identical to the subtype IdA19, a rare subtype that has not been reported previously in BC. To date, the IdA19 subtype has only been identified in 1 sporadic case in Northern Ontario and a number of cases in the 2001 waterborne cryptosporidiosis outbreak in North Battleford, SK. Most reports of the gp60 Id allele belong to the IdA15G1 subtype.

CONCLUSION: As the IbA10G2 subtype has been predominant in previous $C$. hominis outbreak and sporadic cases, these results are significant as they indicate the presence of a new sub-population of C. hominis parasites that has the potential to cause future disease outbreaks. The identification of a rare gp60 IdA19 subtype in all cases of cryptosporidiosis associated with the use of a community aquatic centre was consistent with their exposure history and confirmed that all cases were linked epidemiologically.

\section{P50}

\section{SURVEILLANCE OF MULTI-RESISTANT STREPTOCOCCUS} PNEUMONIAE IN SOUTHERN SASKATCHEWAN

E Nagle*, RR McDonald, GB Horsman, PN Levett

Saskatchewan Disease Control Laboratory, Regina, SK

OBJECTIVES: Multi-resistant Streptococcus pneumoniae have recently emerged as a cause of invasive and non-invasive infections. Similar strains of pneumococci have been isolated sporadically from patients in one region of Saskatchewan. The objective of this study was to characterise these isolates.

METHODS: S. pneumoniae isolates, recovered from January 2005 to November 2007, resistant to penicillin and at least one other class of antibiotic, were serotyped using a cps gene PCR method.

RESULTS: 47 isolates of S. pneumoniae were identified from during the study period. Mean age of patients was 5.4 years (range 6 months -78 years); all except for two patients were aged 8 years or under. The male:female ratio was 1 . Isolates were recovered from three pairs of siblings; from each pair, the isolates were identical. 41 isolates were recovered from upper respiratory tract specimens, 2 from ear specimens and 5 from lower respiratory tract specimens. The following serotypes were identified: 6A/6B (2 isolates), 9V (4), 19A (4), 19F (21) and 23F (16). All isolates were resistant to penicillin, $92 \%$ were resistant to co-trimoxazole and $80 \%$ were resistant to erythromycin. Resistance to other agents was less common. The four isolates of serotype 19A were all isolated in 2007 and were recovered from upper (3) and lower (1) respiratory tract infections. 
Serotype 19A isolates were resistant to more antibiotics than were other isolates. All isolates were susceptible to fluoroquinolone antibiotics and to vancomycin.

CONCLUSIONS: S. pneumoniae strains with multiple antibiotic resistance have been isolated frequently from respiratory tract infections in children in one region of Saskatchewan. In contrast to recently published reports, such isolates represent multiple serotypes. All serotypes, with the exception of 19A, are included within the 7-valent pneumococcal conjugate vaccine.

\section{P51}

GENOTYPING OF MEASLES VIRUS IN CANADA: 2002-2008

J Hiebert*, J Beirnes, G Tipples

National Microbiology Laboratory, Public Health Agency of Canada, Winnipeg, MB

OBJECTIVE: A measles vaccine has been licensed for use in Canada since the 1960s and there is a pan-American initiative to eliminate measles from the Americas. Enhanced surveillance, which includes the case-by-case laboratory confirmation of suspected measles cases, is important for monitoring the status of measles in Canada. The elimination of endemic measles was documented in Canada in 2004. Genotyping of measles virus is important for mapping transmission routes, documenting the elimination of endemic virus strains, and differentiating vaccine from wild-type strains. Molecular epidemiological studies have greatly improved the understanding of the distribution of measles virus strains in various regions of the world. However, continued sampling of measles virus strains is needed for a more complete understanding of their evolving global distribution. We have previously reported the genotypic analysis of wild-type measles viruses in Canada from 1979 through 2002. The objective of this study was to update the genotypic analysis of measles viruses in Canada from 2002 to 2008.

METHODS: Clinical specimens were received from across Canada for measles RT-PCR diagnostics, performed by TaqMan real-time RT-PCR. Specimens that tested positive by real-time RT-PCR were genotyped as per World Health Organization (WHO) standard by amplifying and sequencing the last 456 nucleotides that code for the carboxyl-terminal region of the nucleoprotein $(\mathrm{N})$ gene. Specimen sequence data were analyzed and compared to $\mathrm{WHO}$ reference strains using DNASTAR Lasergene 7 MegAlign program. Genotypes were assigned by homology to reference strains.

RESULTS: Over 60 specimens were genotyped and analyzed. Multiple isolates from the same outbreak and postvaccination genotype A strains have been excluded, leaving 24 sequences. Nine different genotypes were detected: B2, B3, D3, D4, D5, D6, D8, D9 and H1. Eight of these 24 patients had known travel outside of the country.

CONCLUSION: As was found in the earlier study, the wide variety of measles virus genotypes found supports epidemiological data showing that importation of measles is the source of current measles cases in Canada.
P52

DEVELOPMENT OF CANADIAN CLINICAL PREVENTIVE GUIDELINES FOR NEWLY ARRIVING IMMIGRANTS AND REFUGEES: A NATIONAL COLLABORATION TO IMPROVE THE HEALTH OF MIGRANTS

H Swinkels ${ }^{1 *}$, J Feightner ${ }^{2}$, LJ Kirmayer ${ }^{3}$, N Macdonald ${ }^{4}$, L Narasiah ${ }^{5}$, K Pottie $^{6}$, M Rashid ${ }^{7}$, V Robinson ${ }^{8}$, P Tugwell ${ }^{8}$, C Greenaway ${ }^{9}$

${ }^{1}$ Department of Health Care and Epidemiology and Department of Family Practice, University of British Columbia, Vancouver, BC;

${ }^{2}$ Department of Family Medicine, Schulich School of Medicine and Dentistry, University of Western Ontario, Hamilton, ON; ${ }^{3}$ Culture and Mental Health Research Unit, SMBD-Jewish General Hospital, Division of Social \& Transcultural Psychiatry, McGill University, Montreal, QC; ${ }^{4}$ Department of Paediatrics, IWK Health Centre, Dalhousie University, Halifax, NS; ${ }^{5}$ Programme Régional d'Acceuil et d'Intégration des Demandeurs d'Asile (PRAIDA), Montreal, QC; ${ }^{6}$ Department of Family Medicine, Institute of Population Health and Elisabeth Bruyere Research Institute, University of Ottawa, Ottawa, ON; ${ }^{7}$ Access Alliance Community Health Centre, Toronto, ON; ${ }^{8}$ Institute of Population Health, University of Ottawa, Ottawa, ON; ${ }^{9}$ Division of Infectious Diseases, SMBD-Jewish General Hospital, McGill University, Montreal, QC

OBJECTIVES: Immigrants make up an important and growing proportion of the Canadian population. Several subgroups of immigrants have increased mortality from preventable and treatable illnesses. These disparities are likely due to higher prevalence of undetected infectious diseases, low health care utilization and lack of adequate training of Canadian health care workers regarding the priority preventive health needs of this population. There are no clinical guidelines in Canada that address the specific health needs of the immigrant population. The objective of this project will be to develop evidence-based preventive guidelines for immigrants and refugees within the first 5 years of arrival in Canada so that these diseases can be detected and treated and health outcomes improved. METHODS: Using a modified Delphi consensus process, primary care practitioners applied specific criteria to select 20 priority conditions for guideline development. A consensus meeting of 22 experts in immigrant and refugee health (clinicians, epidemiologists, public health specialists, community representatives) developed a systematic 14 step process to identify, synthesize and grade the literature. A standardized search strategy was used and the quality of identified studies assessed with validated tools. For each condition, data was synthesized and clinical recommendations made based on the GRADE approach.

RESULTS: Nine infectious diseases (hepatitis B and C, HIV, intestinal parasites, malaria, MMR/dT, syphilis, tuberculosis, varicella), 4 mental health (abuse and domestic violence, anxiety and adjustment disorder, depression, torture, post traumatic stress disorder) and 7 other conditions (cervical cancer, contraception, diabetes, dental caries, iron deficiency anemia, pregnancy screening, vision screening) were chosen for guideline development. Teams of national and internationally recognized clinicians and researchers have completed the systematic literature reviews and are synthesizing the data. Selected preliminary recommendations will be presented.

CONCLUSION: Targeted clinical preventive health care guidelines will help guide primary care providers identify and manage the most important preventable and treatable diseases in the immigrant population. They may ultimately serve to improve health outcomes in these new Canadians. 
P53

COMPARISON OF CAMPYLOBACTER JEJUNI ISOLATES FROM HUMAN CASES AND CHICKEN IN ONTARIO BY FLA GENE SEQUENCING

G Broukhanski ${ }^{1,2 *}$, R Eshaghi $^{1}$, W Mei ${ }^{3}$, J Labelle ${ }^{1}$, A Valdivieso-

Garcia $^{4}$, S Brown ${ }^{1}$, F Jamieson ${ }^{1,2}$

${ }^{1}$ Toronto Public Health Laboratories, Toronto, $\mathrm{ON}$; ${ }^{2}$ University of Toronto, Toronto, $\mathrm{ON} ;{ }^{3} \mathrm{BC}$ Centre for Disease Control, Vancouver, BC; ${ }^{4}$ Public Health Agency of Canada, Guelph, ON

OBJECTIVES: Campylobacter jejuni $(\mathrm{Cj})$ is the most common cause of gastroenteritis in North America. It is often present in raw foods of animal origin, including poultry. To better understand the role of chickens in its transmission we used sequence typing to determine whether the same subtypes are infecting humans and chicken in Ontario.

METHODS: Cj was isolated from 19 chicken meat samples at the Laboratory for Foodborne Zoonoses, Public Health Agency of Canada and 19 human stool specimens at the Ontario Public Health Laboratories. DNA was extracted and amplified with FLA4F and FLA1728R primers, generating a $1700 \mathrm{bp}$ fragment (total flaA gene template) (Meinersmann et al, 1997). The short variable region (SVR) of flaA was sequenced in both directions using FLA242FU and FLA625RU primers. The resulting sequences were analyzed with BioNumerics software (Applied Maths, Belgium).

RESULTS: Sequencing of SVR fragments demonstrated overlapping nucleotide peaks in 3 chicken $(\mathrm{CI})$ and 2 human isolates (HI), reflecting differences in the two copies of the fla gene present in the $\mathrm{Cj}$ genome. To account for this, sequencing was optimized by varying primers, amplification conditions and concentration of cycle sequencing targets. Pair-wise cluster analysis demonstrated that there were no clusters of $\mathrm{Cj}$ associated only with CI or HI. In contrary, there were 5 clusters with identical SVR sequences: 3 comprising of one $\mathrm{HI}$ and one $\mathrm{CI}, 1$ of $1 \mathrm{HI}$ and $3 \mathrm{CI}$ and 1 of $3 \mathrm{CI}$ and $2 \mathrm{HI}$.

CONCLUSIONS: Cluster analysis of HI and CI shows that there are no unique subtypes circulating only in the human or chicken populations in Ontario. Identical (by SVR sequencing) isolates found in both populations demonstrate genetic stability of circulating $\mathrm{Cj}$ strains. Variability between the 2 copies of the fla gene can be utilized as an additional discriminating factor between strains if primers specific for each copy are used. Use of other typing methods, such as AFLP or MLST, can further determine the molecular epidemiology of clusters of $\mathrm{Cj}$ in humans and chicken.

\section{P54}

\section{CHARACTERIZATION OF HUMAN CEFOXITIN-RESISTANT} (FOX-R) SALMONELLA ISOLATES IN CANADA 2005-2006 L Mataseje ${ }^{1,2 *}$, J Xiao ${ }^{4}$, S Kost ${ }^{1}$, L-K Ng ${ }^{1,2}$, K Dore ${ }^{3}$, M Mulvey ${ }^{1,2}$, and Canadian Integrated Program on Antimicrobial Resistance Surveillance (CIPARS)

${ }^{1}$ University of Manitoba, Winnipeg, MB; ${ }^{2}$ National Microbiology Laboratory, Winnipeg, MB; ${ }^{3}$ Laboratory for Foodborne, Waterborne and Zoonotic Infections Division, Guelph, $\mathrm{ON} ;{ }^{4}$ Centre for Disease Control and Prevention, Shenzhen, Baoan, China; CIPARS

OBJECTIVE: Resistance to extended spectrum cephalosporins (ESC) has increased in Salmonella worldwide since 1999 and is a major concern in both hospital and community settings. The aim of this report was to investigate cefoxitin resistant (FOX-R) Salmonella strains isolated from clinical cases across Canada.

METHODS: All human FOX-R Salmonella strains identified in 2005-2006 through the Canadian Integrated Program for Antimicrobial Resistance Surveillance (CIPARS) were tested for antimicrobial susceptibility using the microbroth dilution method (Sensititre ${ }^{\mathrm{TM}}$ ). FOX-R isolates defined as having an $\mathrm{MIC}>16 \mathrm{mg} / \mathrm{L}$ were screened for the ampC genes DHA, FOX, ENT, CIT in a multiplex PCR followed by sequence analysis. Plasmid analysis by RFLP and replicon typing was performed on a representative subset of FOX-R Salmonella serotypes and phage types (PT).

RESULTS: In 2005 5.4\% (185/3422) and 2006 3.1\% (102/3247) of Salmonella collected from all regions across Canada displayed FOX-R. 84/287 (29.3\%) were multi drug resistant (MDR) as defined as resistant to $\geq 2$ different antibiotic classes. 95.4\% (274/287) of the FOX-R isolates contained the bla $a_{\mathrm{CMY}-2}$ gene. Analysis of CMY-2 plasmids revealed $21.7 \%$ (18/83) contained genes conferring additional resistance. Replicon typing of transformant CMY-2 plasmid DNA revealed the predominance of repI1 (90.7\%) followed by repA/C (16.3\%). $11.6 \%$ of transformants contain $>1$ replicon type. Of the MDR CMY-2 plasmids, $12 / 18$ (67\%) are replicon type repA/C. RFLP patterns of CMY-2 plasmids revealed 39.5\% clustered with $>80 \%$ homology which corresponded to the I1 replicon type.

CONCLUSIONS: Although we observed a decrease in FOX-R from 2005 to 2006 it will be interesting in future studies to monitor the prevalence of Salmonella carrying bla $\mathrm{CMY}_{2}$ on plasmids in Canada. Incompatibility group repI1 is the most prevalent of the CMY-2 plasmids while repA/C is strongly associated with MDR CMY-2 plasmids. Further studies are planned to determine if similar plasmids are circulating in Salmonella identified from food and animal sources.

\section{P55}

\section{THE DETECTION OF WEST NILE VIRUS INFECTIONS IN} HUMANS AND HORSES FROM CUBA: 2003-2005

\section{Andonova ${ }^{1 *}$, M Pupo ${ }^{2}$, M Guzman ${ }^{2}$, R Lindsay ${ }^{1}$, H Artsob ${ }^{1}$,} M Drebot ${ }^{1}$

${ }^{1}$ National Microbiology Laboratory, Winnipeg, MB; ${ }^{2}$ Tropical

Medicine Institute "Pedro Kouri", Havana, Cuba

OBJECTIVES: Since its incursion into North America in 1999 the range of West Nile virus (WNV) has expanded across the continent and into Central and South America. As part of a Canadian and Cuban research collaboration the objective of this study was to identify WNV-associated illness among humans and horses in Cuba.

METHODS: Suspect human cases of WNV were identified based on clinical symptoms associated with neurological disease. Horse samples were collected as part of a WNV surveillance initiative with additional specimens originating from an infectious anemia study. Sera from patients and horses was tested by hemagglutination inhibition (HI) serology and IgM/IgG enzyme-linked immunosorbent assays (ELISA) using WNV antigen generated from mice and tissue culture. Reactive serum samples were further tested by a plaque reduction neutralization test (PRNT) using the North American strain of WNV.

RESULTS: Of 13 Cuban patients diagnosed with encephalitis in 2003, two were confirmed as being WNV cases based upon seroconversions using HI, ELISA, and PRNT diagnostic assays. The cases resided in communities in Santi Spiritus and Villa Clara in central Cuba. Additional exposures to WNV were also identified in both 2004 and 2005, the details concerning clinical symptom presentation and case location will be presented. Over 20 horses from Havana Province were found to be positive for WNV antibodies but displayed no visible signs of illness. A number of human and horse sera were also positive for St. Louis encephalitis virus (SLEV) suggesting that this flavivirus is also contributing to disease in Cuba.

CONCLUSIONS: Our results indicate the co-circulation of WNV and SLEV in Cuba. Further surveillance and timely testing of suspect cases is warranted to determine the risk for exposure to these viruses among residents of Cuba and travellers to this country.

\section{P56}

EVALUATION AND COMPARISON OF COMMERCIAL IMMUNOLOGICAL REAGENTS FOR LEGIONELLA SPP K Bernard $^{1,2}$, A Reimer ${ }^{1 *}$, D Wiebe ${ }^{1}$, T Burdz ${ }^{1}$, B Ng${ }^{1}$ 1Public Health Agency of Canada, Winnipeg, MB; ${ }^{2}$ University of Manitoba, Winnipeg, MB

BACKGROUND: Legionella pneumophila sg 1 (Lp1) is the most common etiological agent causing waterborne disease in Canada; however, $20 \%$ of legionellosis cases are caused by non- $L p 1$ strains. Since 1981, our centre has received 92 isolates of Legionella spp. other than L. pneumophila (Lp) and 342 non-sg1 $L p$ isolates. Inconsistencies in serogrouping results from various kits and cross-reactivity (CR) between sg's of Lp and between nonpneumophila Legionella spp. triggered this investigation. We set out to map the CR of the various antibody preparations and to determine which kits provide better specificity and sensitivity. 
METHODS: The following kits/reagents were used according to manufacturer's instructions: Monofluo Lp IFA (BioRad), FITC conjugates and antigens for 21 strains of $L p$ from 14 serogroups (mTech), FITC monoclonal antibody (MAb) DFA reagents for Lp serogroups 1-14, individual and combined (ProLab), Latex agglutination for Lp serogroups 1 through 14, individual (ProLab), FITC conjugates and antigens for 21 different Legionella spp. other than Lp (mTech), FITC MAb DFA reagent for L. micdadei (ProLab), and Formalin-killed whole-cell antigen preps (In-house). RESULTS \& CONCLUSIONS: Significant CR observed b/w mTech $L p 1 \mathrm{MAb}$ reagents against $L p 1$ strains. Both ProLab and mTech conjugate preps for sg 1-14 are only weakly reactive for many in-house, mTech, and ProLab antigens. ProLab sg12 conjugate did not cross react with any sg1 strains; however, ProLab sg12 conjugate was nonreactive when tested against ProLab sg 1-14 antigen and mTech sg12 antigen. ProLab sg12 conjugate was strongly reactive against in-house sg12 antigen prep. mTech sg12 conjugate was reactive against several strains of sg1. ProLab sg12 conjugate, using in-house antigen prep control, is the best choice to minimize $\mathrm{CR}$ against sg1 strains. Overall mTech reagents were more CR and more sensitive than Prolab reagents. CR was observed amongst sg 2, 3 and 6 and amongst 4, 5, 8, and 9 strains/reagents. Legionella speciation by MAb DFA reagents is possible for some species; however, in most cases at least minor CR with closely related species was observed. Minor-moderate CR was observed b/w strains/reagents of $L$. bozemanii sg1, gormanii, parisiensis, steigerwaltii, and cherrii. Similar CR was observed b/w the strains/reagents of L. erythra, rubrilucens, spiritensis, and taurinensis.

\section{P57}

\section{INVESTIGATION OF AN UNUSUAL GRAM-NEGATIVE BACILLUS RECOVERED FROM CONTAMINATED TOOTHPASTE, IDENTIFIED AS A MEMBER OF THE GENUS HALOTALEA}

K Bernard 1,3*, B Ng1', A Reimer', T Burdz'1, D Wiebe', J Gagnon², C Coignaud $^{2}$, J Bellemare ${ }^{2}$, G Gouin ${ }^{2}$, H Boucher ${ }^{2}$ ${ }^{1}$ National Microbiology Laboratory, Winnipeg, MB; ${ }^{2} \mathrm{HPFBI}$, Longueuil, QC; ${ }^{3}$ University of Manitoba, Winnipeg, MB INTRODUCTION: In 2007, Health Canada's (HC) Health Products and Food Branch Inspectorate (HPFBI) conducted a microbiological investigation of a counterfeit toothpaste product sold in Canada falsely labelled as Colgate $\odot$. Such toothpaste was found to be contaminated with several bacterial types. One bacterial isolate recovered was difficult to identify using standard methods, with the Vitek identification including (risk level 3 agent) Burkholderia pseudomallei among responses. B. pseudomallei was rapidly ruled out by genetic and other testing. Subsequently, the isolate was characterized at the National Microbiology Laboratory (NML). We present here characteristics of the toothpaste isolate; rapid identification of the isolate as part of this investigation was complicated by the fact that the genus Halotalea was only formally described in the literature in September 2007, after testing was completed.

METHODS: After Burkholderia pseudomallei had been rapidly ruled out, 5 colonies of the strain [NML 070603], found to give identical results, were characterized using standard phenotypic, chemotaxonomic and genetic methods. Phylogenetic analysis of the 16SrRNA gene sequence was done to elucidate its relationship with members of the family Halomonadaceae, including Halotalea alkalilenta.

RESULTS AND CONCLUSIONS: The bacterium had 99.7\% identity with Halotalea alkalilenta gen. nov. sp. nov., Genbank accession no. DQ421388 (3 gaps, 2 mismatches). The strain was a strictly aerobic nonfermenter. A number of features which differed from the type strain were observed (was apigmented, oxidase negative, was reactive in OF xylose, mannitol, sucrose and with PPA, but non-reactive in galactose, and melibiose), which may or may not be method associated. Cellular fatty acid composition of the toothpaste strain was similar to that of the type strain of $H$. alkalilenta. This case served to illustrate the critical importance of monitoring of imported goods into Canada and that microbiology laboratories may be called upon to characterize a (possibly widely-disseminated) bacterium with no formal literature description and unknown pathogenic potential for human disease. As well, involvement of a novel taxon group could complicate public health risk assessment and / or compliance actions.

\section{P58}

FOODBORNE BOTULISM IN BRITISH COLUMBIA: A 30 YEAR HISTORY

J Wong ${ }^{1 *}$, J Fung ${ }^{1}$, J Isaac-Renton ${ }^{1,2}$, L McIntyre ${ }^{1}$, A Trinidad ${ }^{1}$ ${ }^{1} \mathrm{BC}$ Centre for Disease Control, Vancouver, BC; ${ }^{2}$ University of British Columbia, Vancouver, BC

OBJECTIVES: Foodborne botulism is a severe neuroparalytic disorder resulting from ingestion of preformed toxin produced during the growth of Clostridium botulinum in contaminated food. Only a few nanograms of the toxin can cause illness which usually presents with gastrointestinal symptoms, blurred or double vision, progressing to dry mouth, dysphagia and symmetrical flaccid paralysis. Death may occur to due to respiratory failure. This summary reviews the cases of foodborne botulism in British Columbia (BC) over the last 30 years, the geographic distribution, the food types involved and the toxins responsible.

METHODS: Collection and submission of clinical samples is coordinated through the BCCDC Laboratory Services, the only laboratory in BC carrying out this testing. Diagnosis of foodborne botulism is confirmed by the demonstration of botulinum toxin or toxin producing C. botulinum organisms in clinical samples (serum, stool, gastric contents and post-mortem samples) or incriminated food. The toxin is detected by a mouse neutralization test. Serum or extracts from food and clinical samples are injected into mice which are then observed for symptoms and death. Toxin type is indicated by neutralization using monovalent antitoxin.

RESULTS: Between 1977-2007, the BCCDC Environmental Microbiology laboratory investigated 22 incidents, involving 80 cases with 4 fatalities. The predominant C. botulinum type was E (68\%), followed by B (23\%) and A (9\%). Two restaurants were involved in large outbreaks. The majority of incidents were caused by home smoked salmon, fermented fish eggs or home canned products. All but one incident was in coastal areas of $\mathrm{BC}$ where $\mathrm{C}$. botulinum type $\mathrm{E}$ predominates. BC's last case of foodborne botulism was in 2001 and has been steadily declining since 1977.

CONCLUSIONS: Efforts made by public health to discourage fermentation and improper smoking of salmon products may be attributed to the decline in C. botulinum cases caused by Type E.

\section{P59}

REFLECTIONS ON HIV: IDENTIFYING NEEDS, IMPROVING LEARNING, OPTIMIZING PATIENT CARE

C Tremblay ${ }^{*}$, P Lin², M-F Deslauriers ${ }^{3}$

${ }^{1}$ University of Montreal Hospital Center, Montreal, QC; ${ }^{2}$ Canadian Heart Research Centre, Toronto, ON; ${ }^{3}$ Pfizer Canada Inc., Kirkland, QC

OBJECTIVE: A multi-staged approach was used with the objective of assessing the learning and educational needs of Canadian physicians specializing in HIV/AIDS.

METHODOLOGY: The needs assessment took place in three distinct stages. The first stage consisted of an in-person focus group session. Among the 15 participants were 13 physicians, one patient organization representative and one pharmacist. During the session, two co-chairs led participants through a novel brainstorming method that allowed them to list any and all topics and areas that they both associated with HIV/AIDS management and deemed important areas where further education and research are needed. They generated four main groups; primary care, HIV management, comorbidities and social issues. For the second stage of the needs assessment, a series of questions were developed using the topics and groups generated during the original focus group session. The questions asked second-stage participants to validate the findings of the first group. The discussion took place via a live web-based platform, which connected participants and co-chairs through their computers and their telephones. The co-chairs led the participants through the validation questions, and the participants used an online polling system to indicate their responses (interest in topics, preferred educational tools, resources of choice, general 
feedback, etc.). This data was captured and displayed back to the participants after they had all entered their responses. The third and final stage of the needs assessment made use of a needs assessment questionnaire that was faxed to 300 Canadian physicians. Some 27 respondents completed and returned the questionnaire, and the results were then tabulated to provide further validation of the topics, areas and resources suggested during the original focus group session. This stage allowed for a broader polling of the physicians in the field. A literature search on this methodology yielded no similar three-step needs assessments performed in the past.

RESULTS: Feedback received during the online validation session and the needs assessment questionnaire closely mirrored the results obtained during the original focus group. The four key topics highlighted were primary care, HIV management, comorbidities and social issues. Specific topics were prioritized within each area. Preferred formats for education and communication were also determined.

\section{P60}

SEROLOGICAL DIAGNOSIS OF SYPHILIS: ENZYME-LINKED IMMUNOSORBENT ASSAY TO MEASURE ANTIBODIES TO INDIVIDUAL RECOMBINANT TREPONEMA PALLIDUM ANTIGENS

A Lau*, I Martin, P Sawatzky, R Tsang

National Microbiology Laboratory, Public Health Agency of Canada, Winnipeg, MB

OBJECTIVE: Recombinant Treponema pallidum protein antigens are now available and widely used in commercial enzyme immunoassays (EIAs). Some of these are being used as an alternative to the conventional screening methods. While such tests are more sensitive and may detect syphilis in all stages of infection, a number of these EIAs use a mixture of recombinant antigens, and will not reveal which antigen(s) is (are) responsible for most of the activities in a positive sample. We therefore aim to establish an in-house quantitative indirect ELISA method that measures human serum antibody responses to four individual $T$. pallidum recombinant protein antigens.

METHODS: From our collection of archived sera, 310 samples were tested and based on a consensus result derived from screening and confirmation tests, these were classified as non-reactive (NR), active infection, past infection, indeterminate, or biological false positive. In addition, 25 sera from presumably secondary syphilis cases were provided by the Ontario Provincial Public Health Laboratory and tested. A commercially available syphilis positive control and negative control were also used. An in-house recombinant $T$. pallidum antigen based ELISA using $T p N 15$, TpN17, TpN44.5 (TmpA), and TpN47 proteins individually as antigens in the assay was developed and used for all samples.

RESULTS: From the samples that were tested, there were 174 'nonreactive' samples, 37 positive (RPR/VDRL $>1: 8$ ) infections, 32 positive (RPR/VDRL $\leq 1: 8)$ infections, 6 past infections, 63 indeterminate samples and 23 biological false positives. The samples classified as positive with RPR/VDRL $>1: 8$ were found to be reactive to all four antigens by our cutoff criteria. Over $95 \%$ of all biological false positive samples were nonreactive to all four antigens, and over $98 \%$ of non-reactive samples were non-reactive to all four antigens as determined by our cut-off criteria. The 'indeterminate' and 'past infection' samples gave varying reactions to the antigens dependent on the individual sample.

CONCLUSIONS: Through our in-house indirect ELISA we are able to determine which antigen(s) is (are) more active for each sample that is tested. Further studies are required to investigate antigen responses using sera from patients from different stages of syphilis infection.

\section{P61}

IMPLEMENTATION AND ANALYSIS OF AN UPDATED MIRU-VNTR METHOD FOR MOLECULAR TYPING OF MYCOBACTERIA TUBERCULOSIS IN MANITOBA S Christianson ${ }^{1 *}$, P Orr ${ }^{2,3}$, E Bellamy ${ }^{1}$, J Wolfe ${ }^{1}$, M Sharma ${ }^{1,3}$ ${ }^{1}$ Public Health Agency of Canada, Winnipeg, MB; ${ }^{2}$ Manitoba Health, Winnipeg, MB; ${ }^{3}$ University of Manitoba, Winnipeg, MB OBJECTIVE: Since 1994, the gold standard for MTBC genotyping has been IS6110-RFLP, a time consuming, demanding and difficult to interpret test. Attempts at developing a VNTR typing method based on 12 Mycobacterial Interspersed Repetitive Units (MIRU) still lacked the discriminatory power of IS6110-RFLP. Recently however, a set of 24 loci with a highly discriminatory subset of 15 loci was introduced. Both are reported to have higher discriminatory power than the original 12 loci system and may exceed that of RFLP in combination with spoligotyping. We are investigating the implementation of the 15 loci method over the 12 loci method in order to improve discriminatory power within the highly homogenous population of Manitoba.

METHODS: A random sample of 243 MTBC isolates from Manitoba that had previously been typed using the 12 loci MIRU-VNTR method was chosen. They were typed according to the MIRU genotyping. Technical Guide available at http://88.198.5.195/MIRU/files/MIRU. VNTRtypingmanualv4-1.pdf

RESULTS: An increase of $24 \%$ in the number of unique patterns occurred when using the 15 loci compared to the 12 loci. Due to the high proportion of identical types in this sample population (60\% of isolates), the test shows a low discriminatory power. Application of the Hunter Gaston discriminatory index (HGDI) showed an increase from 0.622 (12 loci) to 0.667 (15 loci). However, when creating a subset of the sample population that eliminates the predominant type, the HGDI is 0.978 and 0.983 for the 12 and 15 loci respectively. When looking at this common type, the 15 loci method was still unable to differentiate a small number of isolates which were differentiated by RFLP.

CONCLUSIONS: The implementation of a 15 loci based MIRU-VNTR method offers a small improvement in discriminatory power over the traditional 12 loci method. Further investigation of the ability of the 24 MIRU-VNTR loci set or, alternatively, the addition of spoligotyping to differentiate indistinguishable isolates is taking place.

\section{P62}

USE OF INTERFERON-GAMMA ASSAYS IN A SELECTIVE TUBERCULIN TESTED POPULATION AT A VANCOUVER TB CLINIC

Y Simpson ${ }^{1 *}, \mathrm{M} \mathrm{Morshed}^{1,3}, \mathrm{Q}$ Wong $^{1}, \mathrm{G}$ Stark $^{2}$, V Cook $^{2}$, K Elwood $^{2}$, J Isaac-Renton 1,3

${ }^{1}$ Laboratory Services, BC Centre for Disease Control, Vancouver, BC; ${ }^{2}$ TB Control Division, BC Centre for Disease Control, Vancouver, BC; ${ }^{3}$ Department of Pathology and Laboratory Medicine, University of British Columbia, Vancouver, BC OBJECTIVE: Interferon-gamma (IFN- $\gamma$ ) for the diagnosis of latent tuberculosis infection (LTBI) has been proven to be a better test than the standard tuberculin skin test (TST). BCCDC Laboratory Services has the unique advantage of implementing this test because of its close proximity to the BCCDC TB Control Clinic. This enables timely sample collection and testing for IFN- $\gamma$ assays. Our goal was to determine the feasibility of validating and implementing these assays, using clinical data and other existing diagnostic methods in a selective patient population.

METHODS: Cells and plasma were collected in Cell preparation tubes (CPT's, Becton Dickinson) and Quantiferon ${ }^{\circledR}$-TB Gold tubes (Nil, TB Antigen and Mitogen) from a selective TST tested population of 172 patients seen at the BCCDC TB Control Clinic. The test group consisted of 111 females and 61 males ranging from 2 to 88 years of age, from different ethnic backgrounds. The T-SPOT® (Oxford Immunotec, UK) test was performed immediately and the Quantiferon®-TB Gold (Cellestis, USA) test was performed on batches of samples that were stored at $-20^{\circ} \mathrm{C}$ prior to testing.

RESULTS: Of the total 172 patients, 135 had normal $x$-ray findings and 37 exhibited abnormalities on $x$-ray. 88 patients were nonreactive and 29 were reactive in both IFN- $\gamma$ tests. T-SPOT® was reactive in 34 patients where as the Quantiferon ${ }^{\circledR}$ assay was nonreactive and 2 patients had the reverse discordant results. 19 patients had inconclusive or invalid test results on either the T-SPOT or Quantiferon ${ }^{\circledR}$ tests.

CONCLUSIONS: Evaluation of the two IFN- $\gamma$ tests at BCCDC Laboratory Services confirmed the potential for implementation as diagnostic tests for LTBI in selected populations. Use of these tests would minimize the cost of treatment as well as reduce adverse effects as a result of unnecessary treatment. 


\section{Poster Presentation Friday, February 29 Pavilion Ballroom Foyer}

\section{P63 \\ INFECTION CONTROL E-LEARNING: AN ASSESSMENT OF \\ THE EFFECTIVENESS OF AN E-LEARNING MODULE TO TRANSFER KNOWLEDGE ON THE PROPER USE OF PERSONAL PROTECTIVE EQUIPMENT (PPE) THROUGH DIRECT OBSERVATION}

E Bryce $^{1 *}$, B Gamage ${ }^{2}$, C Hon ${ }^{1}$, J Lochang ${ }^{3}$, A Yassi ${ }^{3}$, D Maultsaid ${ }^{1}$, $\mathrm{S} \mathrm{Yu}^{4}$

${ }^{1}$ Vancouver Coastal Health, Vancouver, BC; ${ }^{2}$ Provincial Health Services Authority, Vancouver, BC; ${ }^{3}$ University of British Columbia, Vancouver, BC; ${ }^{4}$ Occupational Health and Safety Agency for Healthcare, Vancouver, BC

OBJECTIVE: Infection control training helps protect health care workers $(\mathrm{HCW})$ and patients from health care associated infections. E-learning modules are an effective method of transferring knowledge. However, it is unknown if that knowledge is reflected in changes in HCW behaviour. The objective of our study was to evaluate the effectiveness of the learning transfer from infection control e-learning modules to health care worker practices using direct observation.

METHODS: The e-learning module under study includes videos designed to teach routine practices, hand hygiene, and proper use of Personal Protective Equipment (PPE). During clinical orientation sessions for new hospital staff, 117 participants were asked to apply either airborne, droplet or contact precautions during mock scenarios. Scenarios were selected randomly by observers and each participant repeated the same scenario prior to and immediately after taking the module. Participants were scored based on their selection, donning, and doffing sequence of PPE.

RESULTS: Eighty-one percent of the participants were nurses; 19\% were allied health staff; $55 \%$ had less than 1 year's work experience in healthcare. Results showed significant improvement in PPE selection ( $p=0.043$ ) in all three scenarios after viewing the module. However, when the selection scenarios were analyzed individually, only the droplet precautions scenario showed significant improvement $(p=0.021)$. Significant improvement was seen in the donning and doffing scores for all three scenarios $(p=0.001)$, both individually and collectively. Additionally, significant improvement in hand hygiene compliance was seen during both donning and doffing of PPE $(\mathrm{p}<0.001)$.

CONCLUSIONS: The e-learning module effectively transfers knowledge on PPE selection and sequence of donning and doffing. More testing is necessary to ascertain if this knowledge is retained over time.

\section{P64}

THE ROLE OF TOROVIRUS IN NOSOCOMIAL VIRAL GASTROENTERITIS AT A LARGE TERTIARY PAEDIATRIC CENTRE

J Gubbay ${ }^{1 *}$, A Al-Rezqi ${ }^{1}$, M Hawkes ${ }^{1}$, L Williams ${ }^{1}$, S Richardson ${ }^{2,3}$, A Matlow ${ }^{1}$

${ }^{1}$ Department of Paediatrics, The Hospital for Sick Children and The University of Toronto, Toronto, ON; ${ }^{2}$ Division of Microbiology, Department of Paediatric Laboratory Medicine, The Hospital for Sick Children, Toronto, ON; ${ }^{3}$ Department of Laboratory Medicine and Pathobiology, The University of Toronto, Toronto, ON

OBJECTIVE: This study was conducted to describe the viral aetiology and epidemiology of nosocomial viral gastroenteritis (NVG) at a tertiary paediatric hospital and identify any changes over the last 2 decades.

METHODS: Retrospective review of all patients with laboratory confirmed NVG at a tertiary paediatric hospital, from January 1, 2004 to December 31, 2005. NVG was defined as onset of diarrhea beyond 48-72 hours of admission. Data were evaluated using descriptive statistics; parametric and non-parametric tests were performed using SPSS software.
RESULTS: 142 episodes of NVG were found among 133 patients, occurring in $0.48 / 100$ admissions. Median age was 2 years; $42 \%$ were under 1 year of age, and $41 \%$ immunocompromised (IC). The most commonly detected pathogen was torovirus ( $67 \%$ of episodes), followed by rotavirus (19\%) and adenovirus (9\%). A greater proportion of children with torovirus NVG (50\%) were IC than with rotavirus NVG (23\%) that were IC $(p=0.014)$. Seventy-five cases $(53 \%)$ were epidemiologically linked in 32 separate clusters (median cluster size 2, range 2 to 4 ). The NVG rate fell from $0.63 / 100$ to $0.22 / 100$ admissions after March, 2005 ( $P<0.001$ ) when enhanced infection control precautions were instituted in response to an outbreak of vancomycin resistant enterococcus (VRE). In previous studies at this institution, NVG rates were 4.5 and 1.3 per 100 admissions in 1985 and 1997-1999, respectively. Torovirus was not detected in NVG in 1985, was responsible for 35\% of all VG in 1993-1995, and 53.5\% of NVG in 1997-1999 at this facility.

CONCLUSIONS: As previously documented, torovirus is the most commonly identified virus in NVG at this tertiary care paediatric centre, and is more common among IC patients. Half of the NVG cases were epidemiologically linked, and a significant reduction occurred after institution of enhanced infection control practices due to an outbreak of VRE. NVG rates have also fallen significantly at this institution since 1985. Improved education and surveillance for NVG should lead to a further reduction in this problem.

\section{P65}

THE STEALTHY ARRIVAL OF SUPER CLOSTRIDIUM DIFFICILE IN NOVA SCOTIA

S Smith $^{1 *}$, J Leblanc ${ }^{2}$, R Davidson ${ }^{1,2}$, K Forward ${ }^{1,2}$

${ }^{1}$ Dalhousie University, Halifax, NS; ${ }^{2}$ Department of Pathology and Laboratory Queen Elizabeth II HSC, Halifax, NS

OBJECTIVE: The clonal dissemination of highly virulent $\mathrm{C}$. difficile (SuperCD) strains has been widely heralded. Increases in morbidity, colectomy rates and mortality often signal the arrival of the strain into a health care facility. Although we did not recognize clinical signs of the arrival of NAP1 strains, we conducted a survey in June-August 2007.

METHODS: We examined strains submitted to the microbiology laboratory or the Capital District Health Authority for cytotoxin assay from both hospitalized patients and those in the community. Stool samples positive for C. difficile cytotoxin were cultured on cycloserine cefoxitin fructose agar. C. difficile isolates were subjected to PCR using primer sets designed to detect $t c d \mathrm{~A}, t c d \mathrm{~B}, t c d \mathrm{C}$, and binary toxin as well as the tpi gene (C. difficile triose phosphate isomerase housekeeping gene; confirmation for C. difficile speciation). Amplicons were resolved by agarose gel electrophoresis. We identified strains with the NAP1 phenotype, i.e. $t c d \mathrm{C}$ partial deletion and the presence of binary toxin. We then analyzed the distribution of the strains between the health care facilities in the Capital Health Care Region.

RESULTS: We characterized 59 individual strains. Of these, 38 (64.4\%) were SuperCD. 24/38 were females vs 12/21 legacy strains. An unrecognized outbreak was identified in one smaller facility (11/13 strains). SuperCD was recognized in each of our larger health care facilities and represented 14/27 strains from the patients in the community.

CONCLUSION: SuperCD has quickly become the predominant CD strain in our health care facilities. When facilities are small, clinical recognition of its presence may be more difficult. Infection control practitioners should not rely on clinical signals, but should, instead, perform molecular characterization to detect outbreaks and limit further spread.

\section{P66}

EVALUATION OF CHROMOGENIC SCREENING MEDIA FOR THE DETECTION OF VANCOMYCIN RESISTANT ENTEROCOCCI

B Lui, M Harris, P Kastner, L Rosmus, J Fuller*

Division of Medical Microbiology, Department of Laboratory Medicine and Pathology, University of Alberta Hospital, Edmonton, AB

OBJECTIVE: Routine screening for vancomycin-resistant enterococci (VRE) is performed on $\mathrm{m}$-Enterococcosel agar ( $\mathrm{m}$-Ent, BD Diagnostics) by 
many clinical laboratories but requires at least $48 \mathrm{~h}$ for presumptive identification of positive (pigmented) colonies. A chromogenic agar, chromID (bioMerieux), is now commercially available and offers the potential for $24 \mathrm{~h}$ identification of VRE. We evaluated the chromID as an alternative to $\mathrm{m}$-Ent for VRE detection from rectal swabs.

METHODS: 200 rectal swabs collected for VRE surveillance in a tertiary care hospital were plated onto $\mathrm{m}$-Ent and chromID agars, incubated at $42^{\circ} \mathrm{C}$ and $37^{\circ} \mathrm{C}$, respectively, and read at $24 \mathrm{~h}$ and $48 \mathrm{~h}$. Control isolates of van $\mathrm{A}, \mathrm{B}$, and $\mathrm{C}$ genotypes were also compared under the above conditions. Results were scored as 'no growth', 'negative' (growth, not pigmented), and 'positive' (pigmented).

RESULTS: Prospective analysis identified 8 VRE-positive (vanA) specimens ( $4 \%$ of total swabs); all 8 were detected at $24 \mathrm{~h}$ on the chromID while 2 and 5 were detected at $24 \mathrm{~h}$ and $48 \mathrm{~h}$, respectively, on m-Ent (1 failed to grow). Only 2 other 'positive' (pigmented) specimens were recovered on $\mathrm{m}$-Ent at $24 \mathrm{~h}$ but the chromID detected 10 'positive' non-VRE specimens $(5 \%)$, primarily gram-positive cocci and yeast. At $48 \mathrm{~h}$ the specificity of $\mathrm{m}$-Ent remained stable but significant 'positive' colonies were recovered from the chromID. Most of this non-specific growth was yeast that could be distinguished based on colony (pseudohyphae) morphology. Of the control isolates, m-Ent detected 3, 5, and 0 , and chromID detected 6,3 , and 0 of the total $6 \operatorname{van} \mathrm{A}, 10 \operatorname{vanB}$, and 5 van $\mathrm{C}$ enterococcal isolates, respectively. All van $\mathrm{A}$ and $\operatorname{van} \mathrm{B}$ control isolates were recovered on each agar by $48 \mathrm{~h}$ of incubation.

CONCLUSIONS: The recovery of vanA VRE from rectal swabs using chromID agar is much faster $(24 \mathrm{~h})$ than that provided by $\mathrm{m}$-Ent. However, from this evaluation a $24 \mathrm{~h}$ incubation of chromID agar cannot reliably recover vanB VRE and additional incubation significantly increases the amount of non-VRE pigmented colonies and subsequent work-load. The utility of chromID compared to $\mathrm{m}$-Ent still requires further study to determine its role as a VRE screening tool.

\section{P67}

UV-VISIBLE MARKER FOR ASSESSING CLEANING COMPLIANCE OF TOILETS: A VALUABLE TOOL FOR MONITORING CLEANING COMPLIANCE OF TOILETS FOR PATIENTS WITH CLOSTRIDIUM DIFFICILE ASSOCIATED DIARRHEA (CDAD)

M Alfa ${ }^{1,3 *}$, N Olson ${ }^{2}$, A Wald ${ }^{2}$

${ }^{1}$ Dept of Medical Microbiology, Winnipeg, MB; ${ }^{2}$ St. Boniface Research Centre, Winnipeg, MB; ${ }^{3}$ Diagnostic Services of Manitoba, Winnipeg, MB

INTRODUCTION: The presence of C. difficile spores in toilets of patients with CDAD is thought to be a reservoir for nosocomial spread of this organism. Increased frequency of toilet cleaning is often used for patients on isolation precautions due to CDAD. However, little is known regarding cleaning compliance of housekeeping staff and how this impacts on the presence of $\mathrm{C}$. difficile spores in toilets.

OBJECTIVE: The objectives were to determine if a UV-visible marker (UVM) could be used to monitor cleaning efficacy in toilets of hospitalized patients, and whether $\mathrm{C}$. difficile spores were adequately removed by the existing twice-daily housekeeping protocol for CDAD.

MATERIALS AND METHODS: Arm 1 consisted of patients who were on isolation precautions for CDAD (twice daily cleaning) and Arm 2 consisted of patients with diarrhea not due to CDAD who were not on isolation (once daily cleaning). Patients were followed prospectively throughout their hospitalization. UVM was applied on a daily basis to toilets and cleaning compliance was scored the following day using a scale of 0 (UVM completely removed) to 3 (UVM residual shows no removal). Rodac plates containing CDMN agar were used to sample the toilet surface in 5 different locations and clostridial isolates detected were assessed to confirm if they were toxigenic C. difficile.

RESULTS: There were 469 and 357 toilet samples taken from patients in Arms 1 and 2 respectively and the average cleaning scores were 1.31 and 1.65 respectively. Toxigenic C. difficile was detected $11.5 \%$ of the time in Arm 1 and $39.7 \%$ of the time in Arm 2. C. difficile was detected $44.3 \%$ of the time when the UVM was 3 and $37.2 \%$ when the UVM was $<3$ in Arm
1. To determine if this poor compliance with cleaning was widespread, all toilets (36 patient rooms) on three different wards were screened for a week. The average cleaning score for all three wards was 1.6 (range 1.5 to 2.5). A UVM score of 3 (i.e. no cleaning) was detected between $42-82 \%$ of the time for these wards.

CONCLUSIONS: Despite the twice daily cleaning, the currently used housekeeping protocol is ineffective in eradicating C. difficile from toilets. UVM is a simple, inexpensive but reliable method to assess compliance of housekeeping staff. We would recommend this tool be implemented as a means of providing feedback to staff regarding cleaning compliance.

\section{P68}

\section{SOAP AND WATER ARE SUPERIOR TO ALCOHOL RUB AND ANTISEPTIC WIPES FOR REMOVAL OF CLOSTRIDIUM DIFFICILE BY HANDWASHING: A RANDOMIZED CONTROLLED TRIAL}

M Oughton ${ }^{1 *}$, V Loo ${ }^{2,3,4}$, N Dendukuri ${ }^{5}$, S Fenn ${ }^{4}$, M Libman $^{2,3,4}$ ${ }^{1}$ Department of Medicine, McGill University, Montreal, QC; ${ }^{2}$ Division of Infectious Diseases, Department of Medicine, McGill University, Montreal, QC; ${ }^{3}$ Department of Microbiology, McGill University, Montreal, QC; ${ }^{4}$ McGill University Health Centre, Montreal, QC; ${ }^{5}$ Department of Epidemiology and Biostatistics, McGill University, Montreal, QC

OBJECTIVE: To compare the efficacy of several common hand hygiene methods for removal of $\mathrm{C}$. difficile.

METHODS: Randomized assignment of handwashing interventions to ten volunteers whose hands were experimentally contaminated by nontoxigenic C. difficile. Paired comparisons of interventions were made for two contamination protocols: "whole hand" and "palmar surface". Interventions studied included plain soap with warm $\left(30^{\circ} \mathrm{C}\right)$ water (WWS), plain soap with cold $\left(15^{\circ} \mathrm{C}\right)$ water (CWS), chlorhexidine containing antiseptic soap with warm water (WWA), antiseptic hand wipes (AHW), alcohol hand rub (AHR) and a "no wash" control (NW). Results were analyzed by a Bayesian approach, using hierarchical models adjusted for multiple observations per subject, left vs. right hand, and location on the palm.

RESULTS: Under the "whole hand" protocol, WWS, CWS and WWA yielded the greatest adjusted mean reductions, followed by AHW (in $\log _{10}$ $\mathrm{CFU} / \mathrm{mL}): \quad 2.14$ (95\% credible interval=1.74-2.54), $1.88 \quad(95 \%$ $\mathrm{CI}=1.48-2.28), 1.51(95 \% \mathrm{CI}=1.12-1.91)$, and $0.57(95 \% \mathrm{CI}=0.17-0.96)$, respectively. AHR, at $0.06(95 \% \mathrm{CI}=-0.34-0.45) \log _{10} \mathrm{CFU} / \mathrm{mL}$, was statistically no different than NW. Under the "palmar surface" protocol, WWA, WWS, and CWS again yielded the greatest mean reductions among those with participants with countable C. difficile colonies, followed by AHW (26.7, 26.6, 26.6, and 21.9 colonies/plate, respectively). AHR demonstrated a small but significant reduction compared to NW. Compared with fingertips, hypothenar $(\mathrm{OR}=10.98,95 \% \mathrm{CI}=1.96-37.65)$ and thenar $(\mathrm{OR}=6.99,95 \% \mathrm{CI}=1.25-23.41)$ surfaces were more likely to remain heavily contaminated with $\mathrm{C}$. difficile after handwashing. CONCLUSIONS: Soap and water showed the greatest efficacy in removing C. difficile by handwashing. Neither water temperature nor chlorhexidine significantly affected the efficacy of handwashing. Soap and water should be used preferentially over alcohol-based hand rubs when contact with C. difficile is probable or likely.

\section{P69}

\section{PSEUDOMONAS BLOOD STREAM INFECTION (BSI);} EPIDEMIOLOGY AND CLINICAL CHARACTERISTICS IN ONE INSTITUTION OVER A SEVEN YEAR PERIOD

\section{A Aljifri*}

University of Alberta Hospital, Edmonton, AB

OBJECTIVE: To describe the epidemiology, antibiotics susceptibility, and prognosis of Pseudomonas spp bacteremia at our institution during a 7 year period.

METHODS: A prospectively collected hospital infection control database was searched for all Pseudomonas spp BSI for the years 2000 through 2006, with 114 patients were identified. Reviews of patient 
charts, electronic health records, and the hospital microbiologic information system were performed.

RESULTS: There were 114 episodes of Pseudomonas BSI, 5.92/10.000 admissions ranging from 12-20/year and no change in frequency over the study period. The mean pediatric patient age was 4 , while the mean adult patient age was $54.68 \%$ were male. ICU (16\%), Plastic Surgery (14\%), General Internal Medicine (12\%), and Pediatrics (11\%) were the most common clinical services. The most common underlying disease was malignancy $(16 \%)$. Pneumonia (33\%) and primary BSI $(30 \%-51 \%$ of which were associated with a central venous catheter) were the most common sources of infection. Pseudomonas aeruginosa (95\%) was the common isolated species. Resistance to ciprofloxacin (23\%) was high. The overall hospital mortality was $32 \%$. Of the 37 fatalities, $20 \%$ died within the first 2 weeks.

CONCLUSION: Pseudomonas aeruginosa blood stream infection is known to be a serious infection with significant patient mortality. The rate of Pseudomonas BSI remained stable over a 7 year period in our tertiary care hospital. An alarming rate of resistance to antibiotics, especially to ciprofloxacin was observed.

\section{P70}

LOW CONCENTRATIONS OF HERPES SIMPLEX VIRUS TYPE 2 (HSV-2) DISPLAY ATYPICAL MELTING TEMPERATURE (TM) PROFILES

J Leblanc*, S Campbell, J Pettipas, T Hatchette, R Davidson QEII Health Sciences Centre, Halifax, NS

OBJECTIVE: Real-time has become a powerful tool for detection and sub-typing of HSV. Using Tm analysis of hybridization probes provided in the LightCycler detection kit, HSV types 1 and 2 can easily be distinguished. However, following implementation of LightCycler PCR in our laboratory, atypical melting curve profiles were occasionally observed for specimens containing low viral titres. We have evaluated the efficacy of Tm profiling at low DNA concentrations.

METHODS: Detection of HSV was performed using the Roche LightCycler HSV 1/2 Detection Kit. Copy number was estimated using a standard curve generated with 10 -fold serial dilutions of linearized plasmid-borne HSV-1 and HSV-2. Serial dilutions of DNA extracted from positive clinical specimens (HSV-1 and HSV-2) were also evaluated. Samples displaying shifted $\mathrm{Tm}$ values (described below) were concentrated in low elution volumes using MinElute PCR Purification Kit (Qiagen). DNA sequencing was performed by Dalgen Microbial Genetics Centre (Halifax, NS).

RESULTS: A reproducible and significant decrease in Tm values was observed for both plasmid-borne HSV-2 DNA and HSV-2 positive clinical specimens at low concentrations; however, no differences were observed at higher concentrations. In contrast, no Tm shift was observed with HSV-1 at equivalent DNA concentrations. When HSV-2 dilutions displaying shifted $\mathrm{Tm}$ values were concentrated and submitted for sequencing, we failed to detect the presence of a mutation. Moreover, Tm values of concentrated samples were restored to normal levels.

CONCLUSIONS: At low viral titres, Tm analysis may not be suitable for genotyping HSV-2. Failure to genotype HSV-2 due to low viral DNA concentrations would be problematic in a clinical setting since characterization of these samples would require use of additional diagnostic methods such as nested PCR and restriction endonuclease digestion, thus increasing sample turnaround times and cost. Although the cause of the apparent Tm shifts remains elusive, melting curve profiles and $\mathrm{Tm}$ values were restored to an acceptable range when the viral DNA was concentrated.

\section{P71}

THE UTILITY OF NESTED PCR AND RESTRICTION ENDONUCLEASE DIGESTION FOR THE GENOTYPING OF HERPES SIMPLEX VIRUS (HSV)

S Campbell*, J Leblanc, J Pettipas, T Hatchette, R Davidson

QEII Health Sciences Centre, Halifax, NS

OBJECTIVE: Molecular methods for detection and sub-typing HSV such as real-time PCR provide a rapid and sensitive alternative to viral culture.
Despite the large benefits of commercially available kits, detection and genotyping of some clinical specimens might be hampered by poor amplification and atypical melting curve profiles. In this study, we developed and evaluated the use of a nested PCR followed by restriction endonuclease digestion to resolve problematic specimens that could not be determined by the instrument software.

METHODS: Detection of HSV was performed using the Roche LightCycler HSV 1/2 Detection Kit. Nested PCR was performed on 84 specimens by modification of methods described in (Rosenberg and Lebon, 1991). Briefly, a second round of PCR using novel internal primers was incorporated prior to restriction endonuclease digestion. The sensitivity of the nested PCR was compared to the LightCycler assay using 10-fold dilutions of HSV-1 and HSV-2 positive specimens and copy number estimated using a standard curve generated with plasmid-borne controls.

RESULTS: Of approximately 3600 clinical specimens submitted for HSV analysis, our nested PCR was required in 83 (2\%) cases. Although our nested PCR was able to resolve most ambiguous results, one limitation of our assay is that nested PCR is unable to resolve mixed infections (1 case). CONCLUSIONS: Our nested PCR provides a sensitive and necessary alternative to real-time PCR when melting curve analysis fails to genotype HSV.

\section{P72}

URACIL-DNA GLYCOSYLASE (UNG) INFLUENCES THE MELTING TEMPERATURE (TM) OF HERPES SIMPLEX VIRUS (HSV) HYBRIDIZATION PROBES

\section{J Leblanc*, J Pettipas, S Campbell, R Davidson, T Hatchette}

QEII Heath Sciences Centre, Halifax, NS

OBJECTIVE: Although the Roche LightCycler platform provides a closed system during PCR set up, false positive results due to amplicon contamination can still pose a significant threat. To prevent contamination, UNG can be incorporated into PCR reactions prior to amplification. We sought to evaluate the influence of UNG on hybridization probes used in a commercially available HSV detection kit.

METHODS: Using template DNA provided in the Roche LightCycler HSV 1/2 Detection Kit or DNA extracted from positive clinical specimens, the influence of UNG was evaluated by treating PCR with increasing concentrations $(0,1,2$ or 4 units) of heat-labile UNG prior to PCR amplification. To examine whether Tm shifts (described below) were attributed to the UNG storage buffer, each component was assayed individually or in combination using concentration simulating those used during addition of 4 units of UNG.

RESULTS: A reproducible concentration-dependent decrease in Tm values (approximately $0.5^{\circ} \mathrm{C}$ per unit of UNG) was observed for both the kit positive control (HSV 1/2) and clinical specimens (HSV-1 and HSV-2) when UNG was added prior to amplification. No significant differences in HSV-1 and HSV-2 Tm values were observed using all components of the buffer or any component alone suggesting the dose-dependent reduction of Tm values is due to UNG itself.

CONCLUSIONS: UNG, and not its buffer components, can influence Tm values in real-time PCR assays. Our results highlight the importance of including equivalent amounts of UNG in PCR reactions for both clinical samples and external standards referenced during analysis.

\section{P73}

INTERNATIONAL STUDY OF INTER-LABORATORY VARIATION OF CYTOMEGALOVIRUS VIRAL LOAD K Martin $^{1 *}$, JK Preiksaitis ${ }^{1,2}$, XL Pang 1,2, JD Fox ${ }^{1,3}$, G Miller ${ }^{4}$, A Caliendo $^{5}$, AST Infectious Disease Community of Practice 1Provincial Public Health Laboratory (Microbiology), AB; ${ }^{2}$ University of Alberta, Edmonton, $A B ;{ }^{3}$ University of Calgary, Calgary, AB; ${ }^{4}$ Vanderbilt University, Nashville, Tennessee, United States; ${ }^{5}$ Emory University School of Medicine, Atlanta, Georgia, United States

PURPOSE: The inter-laboratory variability in quantitative nucleic acid testing (NAT) among laboratories performing Cytomegalovirus (CMV) viral load (VL) for the monitoring of solid organ transplant recipients was studied in this international initiative. 
METHODS: Laboratories recruited through the AST ID community of practice reported results on a panel made up of 12 samples: 2 negatives, 7 samples constructed by diluting purified CMV nucleocapsid stock (replicates at two dilutions) and 3 clinical plasma samples containing mutations in the gB, polymerase and UL-97 genes. The dilutions covered the dynamic range $1.52-5.52 \log _{10}$ copies $/ \mathrm{ml}$ based on electron microscopy count. A survey on methodology used was completed by all participating laboratories. The "standard" used for data analysis was determined by calculation from the mean quantitative VL reported on the nucleocapsid stock by six reference laboratories.

RESULTS: Thirty-five quantitative data sets from 33 laboratories (19 US, 12 Canadian, 2 European) were analyzed: 17 used commercial assays and 18 used "in-house" assays. Reported results on panel samples relative to the "standard" were highly variable. Two false positive results were reported. Significant inter-laboratory variation was observed in both actual and selfreported lower limits of detection (range, 1.0-4.0 $\log _{10}$ copies $/ \mathrm{ml}$ ). The reported range of results on individual samples varied from $2.0 \log _{10}(\mathrm{~min}$ imum) to $4.3 \log _{10}$ (maximum). Variation in results was greatest at low viral loads. Overall, only $62.5 \%$ of all results fell within acceptable standards of variation which were defined as $\pm 0.5 \log _{10}$ copies $/ \mathrm{ml}$ relative to the expected result (geometric mean $\pm 0.50 \log _{10}$ ). Inter-laboratory variability on replicate samples was significantly greater than intra-laboratory variability $(\mathrm{p}<0.0001)$. As a group, commercial assays demonstrated less variability compared to all "in house" assays.

CONCLUSIONS: Significant inter-laboratory variability in quantitative CMV VL assessment was observed. This variation may be impacting patient care and limiting inter-institutional comparisons. Standardization is required to allow meaningful inter-institutional comparison of clinical data which is critical for the evaluation of intervention programs.

\section{P74}

EVALUATION OF TRINITY BIOTECH CAPTIA ${ }^{\mathrm{TM}}$ IGG AND EUROIMMUN IGG ELISA ASSAYS FOR DETERMINING IMMUNITY TO VARICELLA ZOSTER, MEASLES AND MUMPS VIRUSES

DA Johnson ${ }^{1 *}$, SE Dale ${ }^{1,2}$, JB Mahony ${ }^{1,2}$

${ }^{1}$ McMaster University, Hamilton, ON; ${ }^{2}$ St. Joseph's Healthcare, Hamilton, ON

OBJECTIVE: To evaluate the Trinity Biotech Captia ${ }^{\mathrm{TM}}$ IgG ELISA and the Euroimmun IgG ELISA assays for the automated determination of immunity to VZV, Measles and Mumps.

METHODS: Sera previously tested using Bion IFA procedure were tested with Trinity kits using the Adaltis P-Lab Jr. EIA analyzer and with Euroimmun kits using the Euroimmun Analyzer I. ELISA results were compared with IFA results and a combined gold standard of two out of three test results was used. Pools of known negative and positive samples were used for precision testing and linearity testing using both analyzers. RESULTS: For VZV IgG, the sensitivities and specificities were 100\% \& $69 \%$ for Bion IFA, $99 \%$ \& $100 \%$ for Trinity and $97 \%$ \& $100 \%$ for Euroimmun. Within-Lab (between-run) precision results for pooled negative sera had a Coefficient of Variation (CV) of 10\% \& 17\% for Trinity and Euroimmun respectively. Within-Lab precision for pools of low positive sera (results slightly above the cut-off) demonstrated a CV of $8 \%$ and $13 \%$ for Trinity and Euroimmun respectively. Trinity VZV IgG was linear to Immune Status Ratio (ISR) of $>3.0$ and Euroimmun VZV IgG was linear to $5000 \mathrm{mIE} / \mathrm{mL}$. For Measles IgG, the sensitivities and specificities were $100 \%$ \& $75 \%$ for Bion IFA, $92 \%$ \& $100 \%$ for Trinity and $97 \%$ \& $100 \%$ for Euroimmun. Within-Lab precision results for pooled negative sera had a CV of $16 \%$ \& $19 \%$ for Trinity and Euroimmun respectively. Within-Lab precision for pools of low positive sera demonstrated a CV of $15 \%$ and $16 \%$ for Trinity and Euroimmun respectively. Trinity Measles IgG was linear to ISR of 3.0 and Euroimmun Measles $\operatorname{IgG}$ was linear to $5000 \mathrm{IE} / \mathrm{mL}$. For Mumps IgG, the sensitivities and specificities were $100 \%$ \& $63 \%$ for Bion IFA, $100 \%$ \& $94 \%$ for Trinity and $90 \%$ \& $100 \%$ for Euroimmun. Within-Lab precision results for pooled negative sera had a CV of $22 \%$ \& $11 \%$ for Trinity and Euroimmun respectively. Within-Lab precision for pools of low positive sera demonstrated a CV of $16 \%$ and $17 \%$ for Trinity and Euroimmun respectively. Trinity Mumps IgG was linear to ISR of 3.5 and Euroimmun Mumps IgG was linear to $140 \mathrm{RE} / \mathrm{mL}$.

CONCLUSIONS: The two ELISA tests gave comparable results and eliminated the subjective reading of fluorescence intensity required for the Bion IFA method.

\section{P75}

\section{GENOMICS-BASED METHODS FOR THE DETECTION AND WHOLE-GENOME SEQUENCING OF AN UNKNOWN VIRUS}

D Eisler $^{1 *}$, D Lawrence ${ }^{1}$, R Gillies ${ }^{1}$, M Petric ${ }^{1,2}$, P Tang $^{1,2}$

1 British Columbia Centre for Disease Control, Vancouver, BC; ${ }^{2}$ University of British Columbia, Vancouver, BC

BACKGROUND: Conventional methods such as virus culture, electron microscopy, serology and PCR inherently fail to detect novel and uncommon viruses in clinical samples. Genomics-based methods such as high density DNA microarrays or shotgun sequencing are better suited for the detection of these unknown viruses.

OBJECTIVE: A stool sample from a 6-year-old female with gastroenteritis was negative for norovirus by RT-PCR and negative for adenovirus and rotavirus by EIA. Upon examination by electron microscopy, virus-like particles were observed. The objective of this study was to identify the potential viral pathogen through shotgun sequencing of the extracted nucleic acid.

METHODS: The sample was concentrated by ultracentrifugation, treated with DNase, and total nucleic acid was extracted. This was randomly reverse transcribed, amplified and cloned to create a random library. The library was subjected to high throughput sequencing. A single clone containing a 195 -bp fragment with $93 \%$ sequence identity to human parechovirus-1 (HPeV-1) was identified. Using primers based on this sequence, a total of $1.8 \mathrm{~kb}$ of sequence was recovered from the library. The rest of the parechovirus genome ( $\mathrm{HPeV}-\mathrm{BC} 1)$ was cloned and sequenced using RT-PCR with primers based on the HPeV-1 and HPeV-3 genomes.

RESULTS: The $7.3 \mathrm{~kb}$ genome of $\mathrm{HPeV}-\mathrm{BC} 1$ has $93 \%$ sequence identity to $\mathrm{HPeV}-1$. Across the capsid region, $\mathrm{HPeV}-\mathrm{BC} 1$ has $88-97 \%$ nucleotide identity to closely-related human parechoviruses. The 5' UTR and 3' UTR are highly conserved between HPeV-BC1 and other human parechoviruses.

CONCLUSION: For clinical specimens in which viral pathogens are suspected but not detected by all available viral diagnostic tests, shotgun sequencing can be employed to identify unknown viruses. The random library can also be used for whole genome sequencing of any viruses that are detected.

\section{P76}

\section{COMPARISON OF THE QIAGEN QIACUBE® AND THE ROCHE MAGNA PURE COMPACT® FOR THE EXTRACTION OF VIRAL NUCLEIC ACID}

C Kern, L Shaw, T Karnauchow*

Regional Virology Laboratory, Children's Hospital of Eastern

Ontario, Ottawa, ON

OBJECTIVES: Automated extractors decrease the labor needed for nucleic acid (NA) extraction, and improve consistency of NA recovery. The Roche MagNA Pure Compact ${ }^{\circledR}$ (MPC) uses magnetic bead technology, accommodates $\leq 8$ specimens/run, and requires ca. $0.5 \mathrm{~h} / \mathrm{run}$. The Qiagen QIAcube ${ }^{\circledR}(\mathrm{QCu})$ uses silica-based technology, accommodates $\leq 12$ specimens/run, and requires ca. $1.0 \mathrm{~h} / \mathrm{run}$. Using total NA extraction kits, we compared the performance of MPC and QCu for extraction of viral DNA and RNA.

METHODS: Specimens were extracted and tested in duplicate, except Flu (extracted X1). HBV: Archived serum samples $(15[-], 25[+])$ were analyzed. $\mathrm{CMV}: 5$ samples spiked with stock CMV were prepared in pooled blank urine. EV (enterovirus): 9 samples spiked with stock EV were prepared in pooled blank CSF. Flu (influenza A): Archived NP specimens $(13[-], 9[+])$ were analyzed. Validated RT assays were used to evaluate extraction methods (PCR (+/-), $\mathrm{C}_{\mathrm{T}}$ value). 
RESULTS: Except for HBV, all PCR (+/-) results were in agreement. For all samples, mean $\mathrm{C}_{\mathrm{T}}$ values for $\mathrm{QCu}$ specimens were $<$ the mean MPC $\mathrm{C}_{\mathrm{T}}$. $\mathrm{HBV}$ : Three discordant PCR results $(\mathrm{QCu}[+], \mathrm{MPC}[-])$ were observed, all with $\mathrm{C}_{\mathrm{T}} \geq 40$. Three specimens thought to be HBV[-] tested [+] after extraction by both MPC and $\mathrm{QCu}\left(\mathrm{C}_{\mathrm{T}} 36.5\right.$ to 40$)$ and are included as [+] samples in this analysis. Mean $C_{T}(n=50): Q C u C_{T}=$ MPC $-1.2 \mathrm{C}_{\mathrm{T}}$ (range -2.6 to + 3.6). $\underline{\mathrm{CMV}}$ : Mean $\mathrm{C}_{\mathrm{T}}(\mathrm{n}=20)$ : $\mathrm{QCu} \mathrm{C}_{\mathrm{T}}=$ MPC $-2 \mathrm{C}_{\mathrm{T}}$ (range -4.7 to + 5.2). Flu: Mean $\mathrm{C}_{\mathrm{T}}(\mathrm{n}=18): \mathrm{QCu} \mathrm{C}_{\mathrm{T}}=\mathrm{MPC}$ $-1.4 \mathrm{C}_{\mathrm{T}}$ (range \pm 1.4$) . \underline{E n V}$ : Mean $\mathrm{C}_{\mathrm{T}}(\mathrm{n}=18): \mathrm{QCu} \mathrm{C}_{\mathrm{T}}=\mathrm{MPC}-0.9 \mathrm{C}_{\mathrm{T}}$ (range 0.2 to 3.1 ).

CONCLUSIONS: In this study, mean $\mathrm{C}_{\mathrm{T}}$ values of NA extracted by QCu were consistently lower than by MPC $\left(<3 \mathrm{C}_{\mathrm{T}}\right)$, indicating better NA recovery $(<1 \mathrm{log})$. Both instruments were easy to use, but MPC needed less hands-on time, and had a shorter TAT. QCu and MPC are sound options for laboratories dealing with lighter volume NA testing.

\section{P77}

\section{A REAL-TIME PCR ASSAY FOR DETECTION OF HERPES SIMPLEX VIRUS IN CSF USING THE SPARTAN DX ${ }^{\text {TW }}$, A NEW REAL-TIME PCR INSTRUMENT}

\section{Shaw, A Rogaeva, C Harder, T Karnauchow*}

Regional Virology Laboratory, Children's Hospital of Eastern Ontario, Ottawa, Ontario

OBJECTIVES: The Spartan DX ${ }^{\mathrm{TM}}$ (Spartan Bioscience Inc., Ottawa $\mathrm{ON}$ ) is a compact, inexpensive, 4-well real-time PCR (RTPCR) instrument. It uses a 2-temperature reaction cycle that promises more rapid results than conventional RTPCR. The diagnosis of HSV infections of the CNS is a logical application for this instrument: on-demand, non-batched analysis where speed and convenience are critical. We evaluate the performance of a TaqMan ${ }^{\circledR}$-based HSV assay adapted for the Spartan DX.

METHODS: The RTPCR HSV assay used at CHEO ("reference-HSV") was modified for the Spartan DX ("Spartan-HSV"). Replicate testing of CSF spiked with HSV-1 DNA and with HSV-2 DNA (180 copies and 160 copies, respectively; ABi Inc., Columbia, MD) was performed. HSV-1 stock $\left(3.2 \mathrm{TCID}_{50} ; 1 \mathrm{log}>\right.$ the lower limit of detection of reference-HSV) was added to blank CSF, extracted, and tested in same- and separate-day runs. Finally, archived CSF specimens (23 PCR[+], 10 PCR[-]) were tested. Extractions were performed by QIAamp DNA Mini Kit. Specimens were tested in parallel by reference-HSV (ABI 7500; BioRad iCycler). Spartan- and reference-HSV results (PCR [+/-], $\mathrm{C}_{\mathrm{T}}$ ) were compared. RESULTS: Spartan-HSV detected both HSV-1 and HSV-2 DNA: mean $\mathrm{C}_{\mathrm{T}}$ values of samples spiked with 180 copies HSV-1/reaction $(\mathrm{n}=8)$ and with 160 copies HSV-2/reaction ( $n=8$ ), were 35 (range: 34-36). For CSF samples spiked with 3.2 TCID $_{50} \mathrm{HSV}_{-1}(\mathrm{n}=46)$, the mean $\mathrm{C}_{\mathrm{T}}$ was 36.6 (range: 35-39). For all study samples, Spartan and reference test results were in agreement. For clinical specimens, there was no meaningful $\mathrm{C}_{\mathrm{T}}$ difference between methods (Spartan $\mathrm{C}_{\mathrm{T}}=$ reference $\pm 2 \mathrm{C}_{\mathrm{T}}$ ).

CONCLUSIONS: The Spartan-HSV assay is reliable and reproducible, with performance equaling conventional RT HSV PCR. The Spartan DX is an excellent choice for laboratories needing an accurate, affordable, convenient platform to accommodate non-batched "stat" testing, or for smaller laboratories seeking to implement real-time molecular diagnostics in their facilities.

\section{P78}

DEVELOPMENT OF A QUANTITATIVE PCR ASSAY FOR HUMAN ADENOVIRUS

K Adie ${ }^{1 *}$, A Mak ${ }^{1}$, A McNabb ${ }^{1}$, M Petric ${ }^{1,2}$, P Tang ${ }^{1,2}$

${ }^{1}$ British Columbia Centre for Disease Control, Vancouver, BC; ${ }^{2}$ University of British Columbia, Vancouver, BC

OBJECTIVE: Adenoviruses are an increasing cause of severe infections in immunocompromised patients such as those with organ transplantation. There are no clinical signs and symptoms specific to systemic adenovirus infections and the diagnosis depends upon laboratory detection of the virus. We designed a quantitative PCR assay to detect and quantitate adenovirus in blood specimens to monitor the progress of these infections.

METHOD: Primers and probes were designed by aligning hexon gene sequences from the NCBI database and searching for regions of conservation within serotypes 1 to 7,11, 34 and 35. The primers sets were evaluated for specificity for adenovirus in conventional PCR and LightCycler SYBR green assays. The best primer set was used to guide the design of a BHQ/MGB probe in the development of a TaqMan assay for the ABI 7900 Real-Time PCR System. Sequence alignments and primer and probe design were done using the DNA Star package, Primer 3 and ABI Primer Express. The qPCR assay was tested against type strains of adenovirus and clinical specimens to determine its sensitivity and specificity.

RESULTS: The adenovirus qPCR assay has an analytical sensitivity on ATCC strains of less than 1 TCID50. The qPCR assay has a wide dynamic range, accurately quantitating viral loads up to $10^{7}$ TCID50. It can also detect a wide range of adenovirus serotypes in addition to the ones used in the design of the primers and probes.

CONCLUSION: This assay is a rapid and sensitive test for the quantitation of adenovirus in blood. The ability to quantitate adenovirus is important for guiding the clinical management of transplant patients with serious adenovirus infection. Integrating the clinical status of the patient, transplant organ function and the direction of the viral load will enable clinicians to make a better decision on whether to reduce, maintain, or increase immunosuppressive therapy and/or consider initiation of antiviral drugs.

\section{P79}

\section{A COMPARISON OF CELL CULTURE VERSUS REAL TIME PCR (RT-PCR) FOR THE DETECTION OF HSV 1/2 FROM ROUTINE CLINICAL SPECIMENS}

S Campbell ${ }^{1 *}$, J Leblanc ${ }^{1}$, J Pettipas ${ }^{1}$, T Hatchette ${ }^{1,2}$, R Davidson 1,2 ${ }^{1}$ Queen Elizabeth II Health Sciences Centre, Halifax, NS;

${ }^{2}$ Dalhousie University, Halifax, NS

OBJECTIVES: Historically, HSV $1 / 2$ were detected using cell culture. Our laboratory has employed RT-PCR for the detection of HSV $1 / 2$ from CSF specimens for several years, however, we recently switched from cell culture to RT-PCR for the detection of HSV from all specimens submitted to our laboratory. Here we compare cell culture versus RT-PCR for the detection of HSV $1 / 2$.

METHODS: HSV 1/2 were cultured using A549 cells. RT-PCR was performed using the Roche LightCycler HSV 1/2 Detection Kit. Specimens were extracted using the Roche MagNa Pure LC automated instrument. An initial prospective comparison of 300 specimens was performed between Dec 06 and Feb 07. A retrospective comparison was later performed comparing specimens submitted during 2 six month periods of time for culture (Dec 04-July 05 and Dec 05-July06) with another 6 months period for specimens submitted for RT-PCR (Dec 06-July 07).

RESULTS: Based on our initial prospective study, RT-PCR detected $13.9 \%$ more HSV than did culture. During the retrospective comparison, 5172 specimens were submitted for culture; $32.4 \%$ were positive for HSV. Of 2217 specimens submitted for RT-PCR, 36.6\% were positive. Overall, RT-PCR detected $11.5 \%$ more HSV than did culture. Based on our analysis, RT-PCR would have likely detected 217 more patients with HSV than did culture over the study period. The observed increase in our detection rate was seen for both HSV-1 and HSV-2. RT-PCR for routine HSV detection adds approximately $\$ 12,000$ to our budget or about $\$ 55$ per additional positive patient. RT-PCR allows same day results compared to a minimum of 48 hours for a positive culture result.

CONCLUSIONS: RT-PCR is a more rapid and sensitive test for the detection of HSV $1 / 2$ compared to culture. Although more expensive, the added sensitivity justifies the additional cost. 


\section{P80}

\section{CLINICAL CONCORDANCE OF THE CDC AND SIEMENS MEDICAL SOLUTIONS DIAGNOSTICS INFLUENZA A/B VIRUS MOLECULAR ASSAYS}

N Kreiswirth ${ }^{1}$, BM Willey ${ }^{1,2}$, J Detmer ${ }^{3}$, S Sangha $^{3}$, M Albalos ${ }^{3}$, A Petrich ${ }^{4}, \mathrm{~S}$ Chong 4 , K Luinstra ${ }^{4}, \mathrm{G}$ Moussa1, C Bush ${ }^{3}$, J Mahoney ${ }^{4}$, S Richardson 5,6, M Smieja ${ }^{4}$, T Mazzulli,2,6, A Tyler ${ }^{1 *}$ ${ }^{1}$ Mount Sinai Hospital, Toronto, ON; ${ }^{2}$ University Health Network Toronto, ON; ${ }^{3}$ Siemens Medical Solutions Diagnostics, Tarrytown, New York, United States; ${ }^{4}$ St. Joseph's Hospital, McMaster University, Hamilton, ON; ${ }^{5}$ Hospital for Sick Children, Toronto, ON, ${ }^{6}$ University of Toronto, Toronto, ON

OBJECTIVES: Seasonal influenza infects approximately $5-20 \%$ of the North American population annually. Although most infections resolve without incident, more than 225,000 are hospitalized and 40,000 die each year. There is need for a reliable, rapid and sensitive clinical assay to detect seasonal influenza and prepare for a potential avian influenza pandemic. As part of a broader study to compare the performance of several in-house and commercial influenza A detection assays available in Canada, the Siemens prototype Influenza A and B (INFA/B) assay was compared to the INFA CDC "gold standard" molecular assay.

METHODS: The Siemens prototype INF A/B assay is an internally controlled real-time RT-PCR assay that can be used to simultaneously amplify and identify multiple strains of influenza subtypes A/B within 4 hours. To demonstrate concordance, both assays were tested and run using the same blinded sample panel at the Univ. of Toronto. The panel was comprised of 44 extracted RNA samples including ten-fold dilutions each of cultured $\mathrm{H} 1, \mathrm{H} 3$ and $\mathrm{H} 5$ viral lysates and one sample each of influenza A H9, inf. B, RSV, hMPV, enterovirus, and three water controls.

RESULTS: Of the 44 blinded RNA extracts tested, both assays detected $37 / 37(100 \%)$ of the diluted influenza A positive samples including the $\mathrm{H} 1, \mathrm{H} 3$, H5 and H9 hem-agglutinin types, demonstrating 100\% concordance between the two assays. In addition, the influenza B positive was identified by the Siemens prototype INFA/B real time RT-PCR assay. The remaining non-influenza samples tested negative by both assays.

CONCLUSION: Comparing results from both assays demonstrates 100\% concordance between the Siemens prototype INFA/B assay and the CDC gold standard protocol.

\section{P81}

DETECTION OF MRSA BY THE BD GENOHM MRSA ASSAY (BMA) FROM FLOCKED SWABS TRANSPORTED IN LIQUID AMIES (LA)

D Goldfarb ${ }^{3 *}$, P Jessamine 1,2, A Bonneau1, K Ramotar ${ }^{1,2}$, M Desjardins ${ }^{1,3}$

${ }^{1}$ The Ottawa Hospital, Ottawa, ON; ${ }^{2}$ The Ottawa Hospital Research Institute, Ottawa, ON; ${ }^{3}$ Children's Hospital of Eastern Ontario, Ottawa, ON

BACKGROUND: MRSA screening by culture requires 48-72 hours compared to $1 \mathrm{hr}$ with the BMA. At the Ottawa Hospital, nasal and rectal swabs are pooled in a selective broth prior to testing. To improve turnaround times (TAT) we evaluated the performance of the BMA using a flocked swab and a LA transport system.

METHOD: Flocked swabs dipped in a 0.5 McFarland suspension of CMRSA-2, CMRSA-7 and CMRSA-10 isolates were placed in the LA and incubated at room temperature (RT) for $1 \mathrm{hr}, 2 \mathrm{hr}$, and overnight. To simulate a rectal swab, CMRSA-2 an inoculated swab was dipped in an MRSA negative stool and incubated in LA at RT for $1 \mathrm{hr}, 2 \mathrm{hr}$ and overnight. For the simulated nasal swab, nares of a negative volunteer were swabbed prior to dipping in a CMRSA-2 suspension and incubated $1 \mathrm{hr}$, $2 \mathrm{hr}$ and overnight at RT. For BMA testing, swabs were vortexed in the LA and a 50ul aliquot was transferred to the sample diluent buffer and processed according to manufacturer's instructions. All runs were performed in triplicate.

RESULTS: All inoculated flocked swabs transported in LA including simulated rectal and nasal specimens were detected by BMA. None of the tests were found to be inhibited.
CONCLUSION: Flocked swabs transported in LA have the potential to be used with the BMA for screening of MRSA colonized patients. Pooling of nasal and rectal specimens collected on flocked swabs and transported in LA could be used with the BMA for direct testing instead of using selective broth and thus potentially reducing TAT. Further evaluations are on going to determine the feasibility and performance of the BMA from nasal and rectal specimens collected on flocked swabs and pooled in LA.

\section{Clinical Vignette Posters Friday, February 29 Pavilion Ballroom Foyer}

\section{CVP1}

\section{THE CASE OF THE FLOPPY BABY}

N Morgan*, A Wadhwa

McMaster University, Department of Infectious Disease/Medical Microbiology

OBJECTIVE: To describe a case of infant botulism occurring in a six month old Caucasian female infant and to review the necessary steps for a timely diagnosis and treatment.

METHODS: Infant botulism is a rare occurrence in Canada. Even when there is a high index of suspicion, there may be delays in diagnosis and treatment due to the uncertainty regarding correct collection and processing of samples, and the procedure for obtaining botulinum immune globulin. The clinical presentation will be reviewed, including the appropriate steps for notification of public health officials, diagnosis and treatment.

RESULTS: A six month old Caucasian female infant presented to hospital with a one week history of generalized weakness of upper and lower limbs, poor sucking and ptosis. She had been exclusively breast fed, until one month prior, when rice cereal was introduced into her diet. Nine days following the initiation of cereal, the infant developed constipation. Ten days later, her parents brought her to hospital when she developed a weak cry and was unable to sit or roll over. Blood and stool specimens were obtained and sent for botulinum toxin. The stool sample subsequently was positive for C. Botulinum subtype B. The cereal also tested positive for C. Botulinum subtypes B and F. She received one dose of botulinum immune globulin. She went on to full recovery and was discharged home two weeks later.

DISCUSSION: Infant botulism may not present a diagnostic dilemma to infectious disease clinicians. However, due to its rare occurrence there may be confusion regarding the appropriate collection of specimens, ordering of laboratory tests, and notification of appropriate public health officials. Treatment involves notification of the Ministry of Health and obtaining immune globulin from California. After presentation of a case of infant botulism, we review the appropriate steps for diagnosis and treatment.

\section{CVP2}

\section{A GRANULOMATOUS BREAST MASS}

\section{DR Ricciuto*, WL Gold}

University of Toronto, Toronto, ON

CASE PRESENTATION: A 28-year-old woman was referred to the Infectious Diseases consultation service for the evaluation of an inflammatory left breast mass. Six weeks prior, she noticed the onset of tenderness and erythema of the left breast. The lesion progressed to the development of 2 localized masses which gradually consolidated into 1 large retroareolar mass. She was born in Canada. Her travel history included a trip to the Philippines several years earlier. She had no sick contacts. Her only pet was a ferret. She was afebrile. The remaining vital signs were also normal. There was an area of skin lateral and inferior to the left areola that was erythematous and edematous with a draining sinus inferior to the areolar margin. Enlarged lymph nodes were palpated in the left axilla.

INVESTIGATIONS: A left breast ultrasound revealed a predominantly solid mass with echogenic fluid within it that measured $2.9 \times 3.5 \times 2.2 \mathrm{~cm}$. Excisional biopsy of the mass revealed granulation tissue showing acute and chronic inflammation with microabscesses, focal granulomatous 
inflammation and multinucleated giant cells. Ductal or lobular structures were not identified. Ziehl-Neelson staining for acid-fast bacilli and Gomori methanamine silver and periodic acid Schiff staining for fungal elements were negative. Further testing including culture and sensitivity of the biopsy samples for bacteria, acid-fast bacilli and fungus were negative. Serology for Bartonella henselae was also negative.

DIAGNOSIS AND DISCUSSION: A diagnosis of idiopathic granulomatous mastititis was made. The patient was treated with a slow, tapering course of oral prednisone with resolution of the lesion. Granulomatous mastitis is a rare, but clinically imporant entity associated with significant morbidity. By mimicking inflammatory carcinoma of the breast, many women undergo unnecessary mastectomy. Furthermore, many Infectious Diseases specialists are consulted on patients with pathological changes that reveal granulomatous inflammation. A broad differential must be entertained in addition to typical infectious agents such as tuberculosis. When granulomatous changes are identified in a breast tissue biopsy a wide differential diagnosis exists, including: duct ectasis; autoimmune processes; other inflammatory conditions; and, infections. Tuberculosis is the most common infectious etiology of granulomatous mastitis, accounting for a significant proportion of breast masses in endemic nations. Other reported infectious etiologies include fungi (blastomycosis, coccidiomycosis and histoplasmosis), Actinomyces spp., Bartonlla henselae, Corynebacterium spp., and filarial infection. In this case, careful exclusion of infectious etiologies allowed for the safe and successful administration of steroid therapy. Further diagnostic testing and treatment was performed.

\section{CVP3 \\ CASE OF BORDETELLA BRONCHISEPTICA AND LEGIONELLA PNEUMOPHILA PNEUMONIA IN AN IMMUNOCOMPROMISED PATIENT \\ B Meatherall*, D Gregson, D Church Department of Infectious Disease, University of Calgary, Calgary, AB}

OBJECTIVE: Bordetella bronchiseptica is the agent responsible for kennel cough in dogs and has been known to cause disease in humans. Several case reports exist in the literature involving AIDS patients as well as solid and non-solid organ transplant recipients. Legionella pneumophila is a cause of pneumonia in both immunocompetent and immunocompromised patients. We describe a case of pneumonia in an immunocompromised patient involving both Bordetella bronchiseptica and Legionella pneumophila. METHODS: A case of a 59 year old female with acute myelogenous leukemia presented 13 months post-allogenic bone marrow transplant with recurrent pneumonia. She was admitted to hospital with her fourth episode of pneumonia in a 5 month period. She was tachycardic, tachypnic and hypoxic on admission. Her laboratory studies were significant for lymphopenia and thrombocytopenia. Blood cultures were negative. Her chest X-ray revealed bilateral upper and lower lobe pulmonary infiltrates as well as a right upper lobe crescentic lesion. She denied significant travel history but had three pet dogs at home. She underwent a bronchoalveolar lavage (BAL) for diagnostic purposes.

RESULTS: Bordetella bronchiseptica was isolated from the BAL specimen at 24 hours of incubation and confirmed by DNA sequencing. Legionella pneumophila was identified on buffered charcoal yeast extract (BCYE) media at 120 hours of incubation. A BAL specimen from 3 months prior revealed a Cryptococcus non-neoformans species, no Legionella or Bordetella species were identified at that time. She was treated in hospital with piperacillin tazobactam, voriconazole, levofloxacin and vancomycin. She died of respiratory failure on day 4 of admission.

CONCLUSION: Bordetella bronchiseptica is an uncommon pathogen in humans, however, it is a may cause significant morbidity and mortality in immunocompromised hosts. Risk factors for transmission include close contact with animals as well as nosocomial contacts. Immunocompromised patients should be counselled with regards to animal/pet exposure. Co-infection is also a possibility in immunocompromised patients and care must be taken to ensure all organisms are appropriately identified. This is the first case reported of laboratory confirmed co-infection with Legionella pneumophila and Bordetella bronchiseptica in the literature to date.

\section{CVP4}

NEW SEIZURES IN A PREVIOUSLY HEALTHY YOUNG MAN - AN UNEXPECTED CASE OF NEUROCYSTICERCOSIS JY Gabor*, JM Embil

Department of Medicine and Medical Microbiology \& Faculty of Medicine, University of Manitoba, Winnipeg, MB

PRESENTATION: A 27-year-old male presented to the emergency department with two episodes described as "seizures". There was no history of infectious prodrome or contacts. He did not have a significant past medical history, did not take any medications, and lacked risk factors for infection with the Human Immunodeficiency Virus (HIV). The patient is employed as a truck driver. He denied recent travel out of Canada, however he had immigrated to Canada from Mexico during his childhood.

INVESTIGATIONS: The patient was afebrile. Neurological examination was significant for confusion, dysarthria, myoclonus of the right leg, and diminished reflexes and strength in the right upper extremity. The remainder of the physical examination was non-contributory. Serum biochemical profile was normal. The leukocyte count was elevated at $13.4 \times 10^{9} \mathrm{cells} / \mathrm{L}$ with a neutrophil predominance. Computed axial tomographic (CT)-scan of the brain demonstrated the presence of numerous small cysts along the subarachnoid spaces of the convexities of both cerebral hemispheres, with most of the cysts demonstrating a central dot of high attenuation. Several intraparenchymal cysts were also identified in both cerebral hemispheres and two lesions in the left parietal lobe were associated with minimal enhancement and adjacent edema. The radiographic findings were most consistent with neurocysticercosis.

TREATMENT: Lorazepam was administered to control agitation and seizure activity. No further seizures were observed. The patient was prescribed phenytoin $200 \mathrm{mg}$ twice daily, dexamethasone $4 \mathrm{mg}$ daily, and a 15-day course of praziquantel, $50 \mathrm{mg} / \mathrm{kg}$.

FOLLOW-UP: Serology was positive for T. Solium. The patient's neurological deficits resolved promptly with treatment. No subsequent seizures have occurred and the patient remains asymptomatic. Repeat CT-scans at two weeks and four months after presentation continue to demonstrate regression of the lesions.

\section{CVP5}

\section{CHRONIC INTERMITTENT HEMATURIA IN A NEW IMMIGRANT CHILD}

\section{A Haleis*, M Alfa, K Manickam, A Ronald}

Department of Medical Microbiology and Infectious Diseases, Faculty of Medicine, University of Manitoba, Winnipeg, MB

An 11 year old male emigrated from Mozambique and arrived in Canada in May 2006. He was born in a refugee camp in Tanzania where he spent five years and he spent the next five years in another refugee camp in Mozambique. In August 2006 he had an episode of gross (painless) hematuria and dysuria. He also gave history of enuresis during the day time. No history of fever or abdominal pain. No history of puffiness around his eyes or generalized edema. Past medical history: malaria; history of exposure to tuberculosis, gastrointestinal parasites and malaria in refugee camp.

On December 2006 seen by pediatrician for ongoing painless hematuria. On exam: BP 114/50; temp 36.8; PR 76/min; weight $29.3 \mathrm{~kg}$ (25 $5^{\text {th }}$ percentile) and height $129 \mathrm{~cm}$ (10 mal.

Laboratory investigations: WCB 4.6 (eosinophils 11\%); Hb 125; plt 262.

Urine analysis:

\begin{tabular}{ll}
\hline Specific gravity & 1.013 \\
$\mathrm{Ph}$ & $6-6.5$ \\
Protein & $1-5 \mathrm{~g} / \mathrm{l}$ \\
Glucose & Negative \\
Ketones & Negative \\
Bilirubin & Negative \\
Hemoglobin & +4 \\
Leukocyte esterase & +1 \\
RBC & $>100$ \\
WBC & $21-50$ \\
Squamous epithelial & $1-2$ \\
\hline
\end{tabular}


Urine culture no growth: C3 and C4 normal; p and c ANCA were negative; Antinuclear antibodies negative; urine protein/creatinine ratio normal. Malaria thin and thick smear was positive for Plasmodium falciparum macrogametocytes. Stool for ova and parasite was negative. Urine for ova and parasite was positive for Schistosoma haematobium.

Patient treated with Praziquantel $40 \mathrm{mg} / \mathrm{kg} /$ day one dose and quinine $30 \mathrm{mg} / \mathrm{kg} /$ day divided tid and clindamycin $30 \mathrm{mg} / \mathrm{kg} /$ day divided tid for 5 days.

\section{CVP6}

FIRST CASE OF MENINGITIS CAUSED BY STREPTOCOCCUS SUIS SEROTYPE 14 IN NORTH AMERICA

A Haleis, M Alfa, K Manickam, A Ronald

Department of Medical Microbiology and Infectious Diseases, Faculty of Medicine, University of Manitoba, Winnipeg, MB

Streptococcus suis is Gram positive, facultatively anaerobic, coccid or ovoid in shape and arranged in pairs or in short chains. It is mainly a swine pathogen. It can cause many diseases in swine including arthritis, meningitis, pneumonia, septicemia, endocarditis, polyserositis, abortions and abscesses. Based on the capsular polysaccharide 35 serotypes have been identified (types 1-34 and 1/2). Serotype 2 is the most virulent and frequently isolated in both swine and humans. Infection in humans is considered an occupational disease that affects persons who work in close contact with pigs or pork by-products. We report a case of human meningitis caused by Streptococcus suis serotype 14. A 59 year old female from rural Manitoba worked in a hog sorting plant sorting piglets. She presented with a 2-day history of fever, vomiting, headache and neck pain and was hospitalized. Her white cell count was 19,900 per cubic millimeter. Cerebrospinal fluid showed leukocyte count of $284 \times 10^{6} / \mathrm{L}$, with $59 \%$ lymphocytes and $41 \%$ polymorphonuclear cells; glucose $2.3 \mathrm{mmol} / \mathrm{L}$ and a total protein $1.85 \mathrm{~g} / \mathrm{L}$. Gram stain of CSF showed gram-positive cocci in pairs. Blood cultures were negative. CSF culture grew alpha hemolytic colonies on blood agar and chocolate agar. The organism was catalase negative and identified as Streptococcus suis by Vitek GPI card (Biomerieux, France) and API Strep System. Streptococcus suis was confirmed by $16 \mathrm{~S}$ ribosomal sequencing. The organism was sensitive to penicillin. Empiric antibiotic treatment with cefotaxime and vancomycin was switched to penicillin G. Her recovery was complicated by bilateral deafness and difficulties with balance; one month later there had been some improvement but she still has bilateral deafness. 


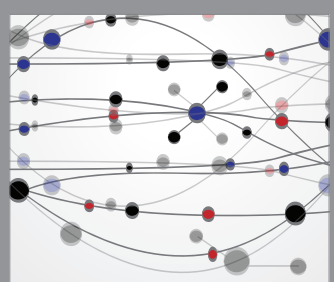

The Scientific World Journal
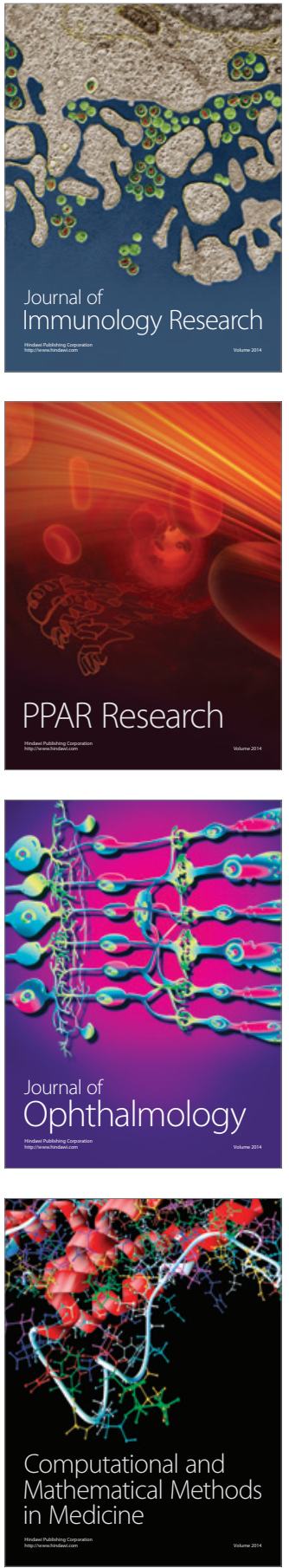

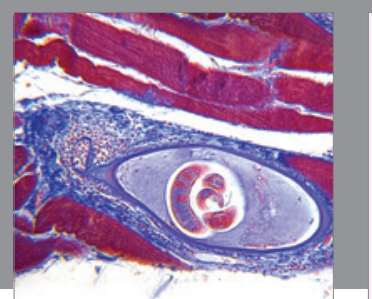

Gastroenterology Research and Practice

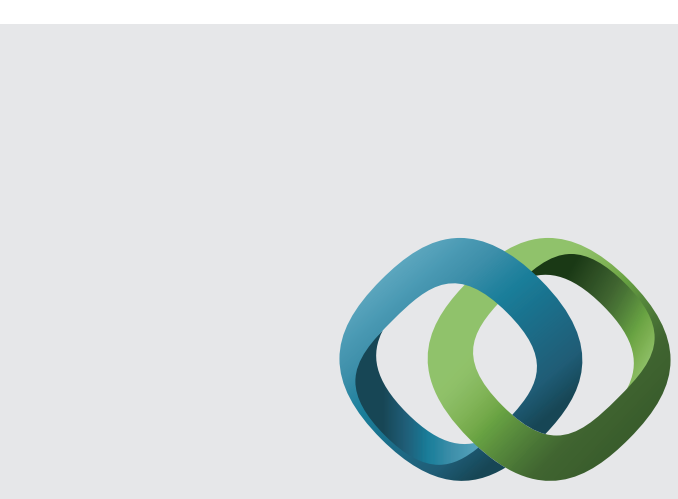

\section{Hindawi}

Submit your manuscripts at

http://www.hindawi.com
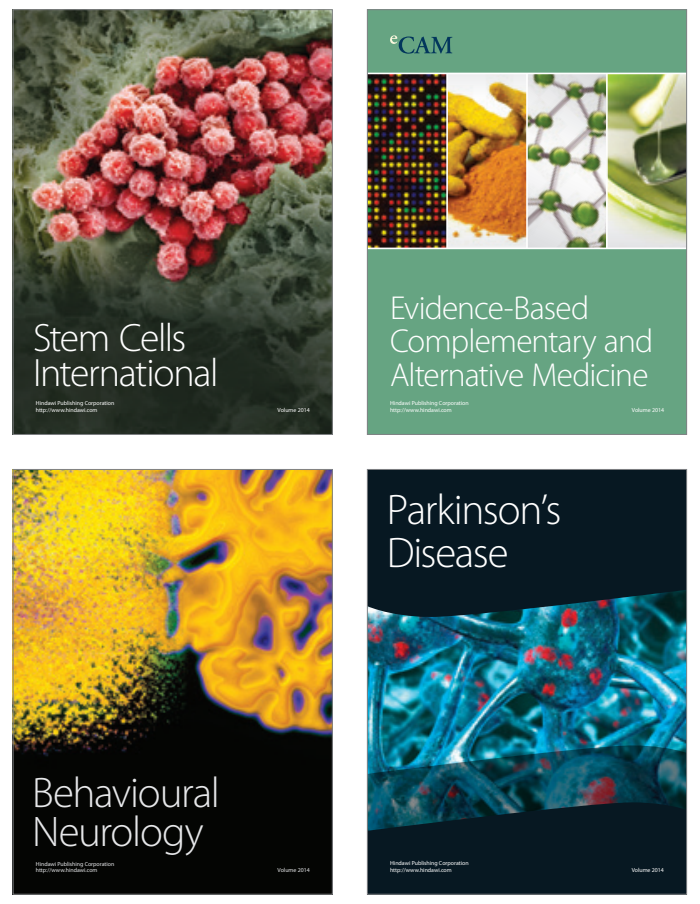
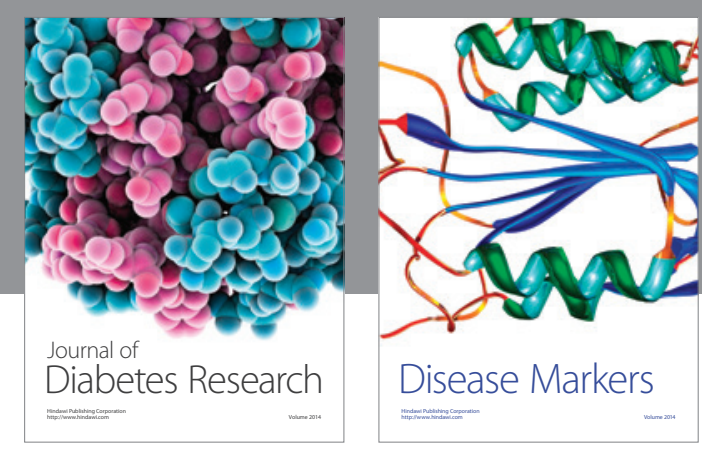

Disease Markers
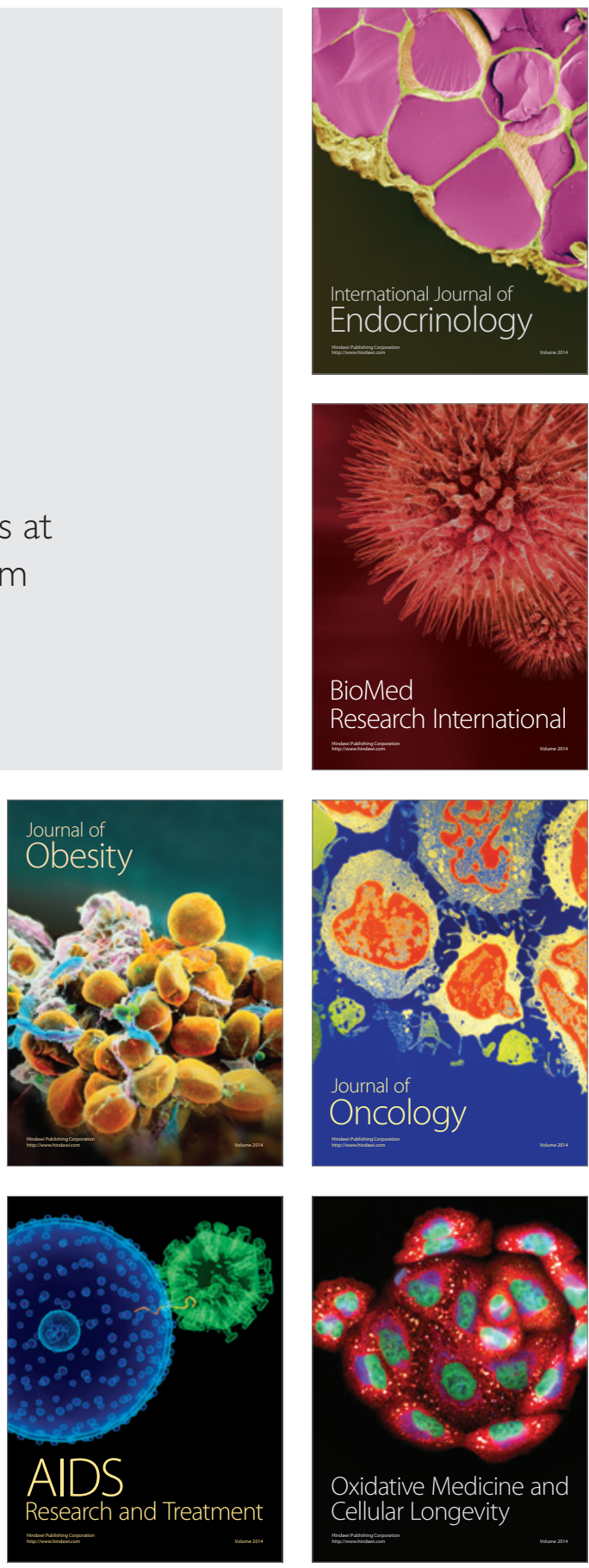